This item was submitted to Loughborough's Research Repository by the author.

Items in Figshare are protected by copyright, with all rights reserved, unless otherwise indicated.

\title{
Investigating the role of redundancy in multimodal input systems
}

PLEASE CITE THE PUBLISHED VERSION

PUBLISHER

(C) K.C. McKenzie Mills

\section{PUBLISHER STATEMENT}

This work is made available according to the conditions of the Creative Commons Attribution-NonCommercialNoDerivatives 2.5 Generic (CC BY-NC-ND 2.5) licence. Full details of this licence are available at: http://creativecommons.org/licenses/by-nc-nd/2.5/

\section{LICENCE}

CC BY-NC-ND 2.5

\section{REPOSITORY RECORD}

Mills, Karen C. McKenzie. 2019. "Investigating the Role of Redundancy in Multimodal Input Systems". figshare. https://hdl.handle.net/2134/28236. 


\section{Loughborough University}

This item was submitted to Loughborough University as a PhD thesis by the author and is made available in the Institutional Repository

(https://dspace.lboro.ac.uk/) under the following Creative Commons Licence conditions.

\section{cc) creative}

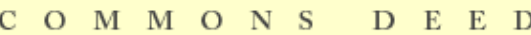

Attribution-NonCommercial-NoDerivs 2.5

You are free:

- to copy, distribute, display, and perform the work

Under the following conditions:

Attribution. You must attribute the work in the manner specified by the author or licensor.

Noncommercial. You may not use this work for commercial purposes.

No Derivative Works. You may not alter, transform, or build upon this work.

- For any reuse or distribution, you must make clear to others the license terms of this work.

- Any of these conditions can be waived if you get permission from the copyright holder.

Your fair use and other rights are in no way affected by the above.

This is a human-readable summary of the Leqal Code (the full license).

Disclaimer 민

For the full text of this licence, please go to: http://creativecommons.org/licenses/by-nc-nd/2.5/ 


\section{Pilkington Library}

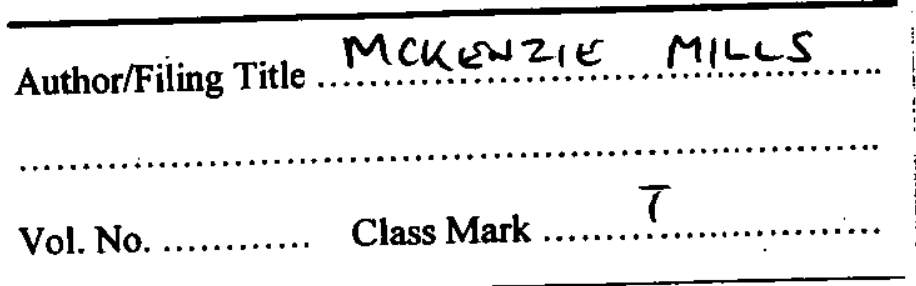

Please note that fines are charged on ALL overdue items.
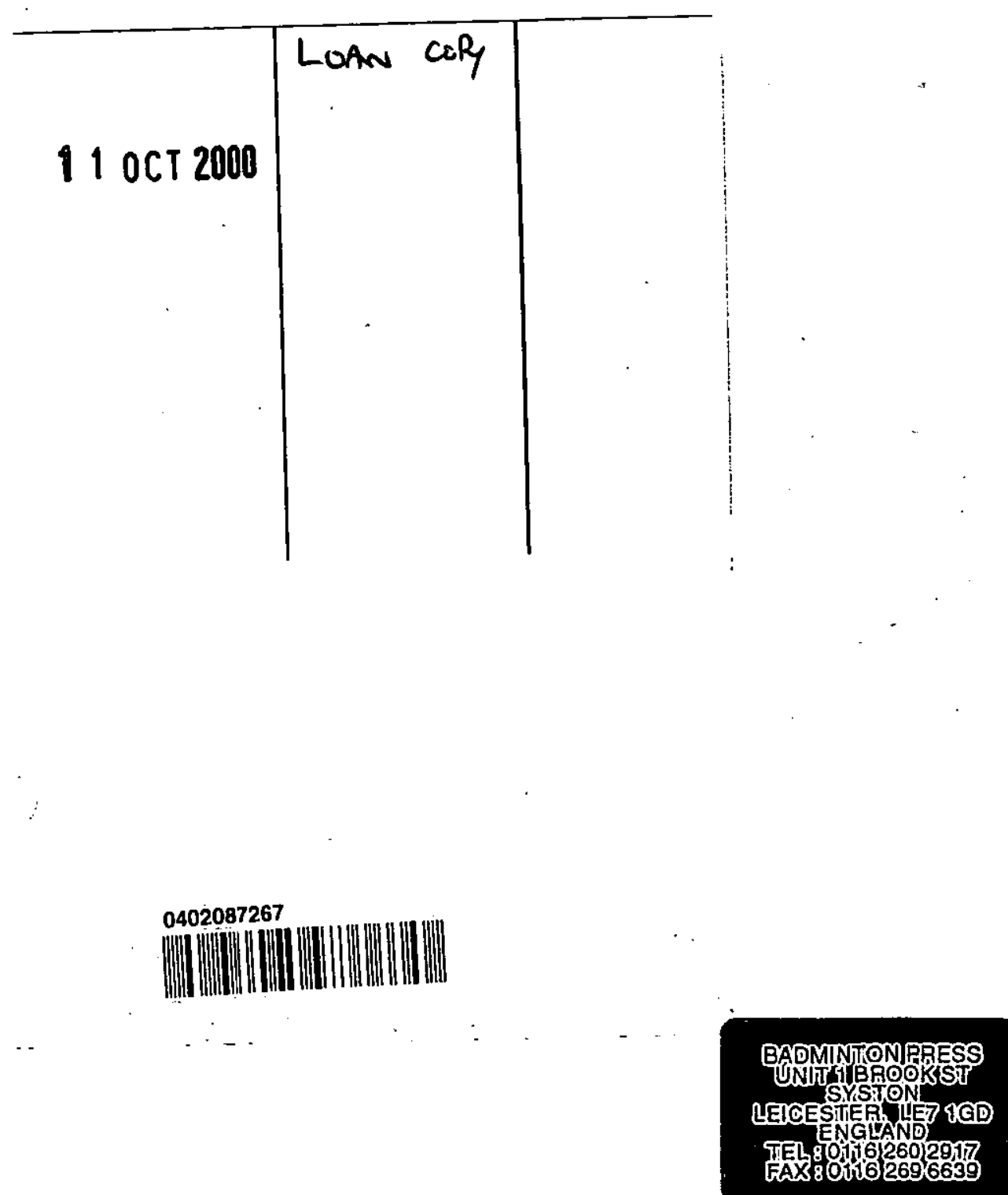


\title{
Investigating the Role of Redundancy in Multimodal Input Systems
}

\author{
By \\ Karen C. McKenzie Mills \\ B.Sc. (Hons.) (Glasgow) \\ M.Sc. (Strathelyde)
}

A Doctoral Thesis

Submitted in partial fulfilment of the requirements

for the award of

Doctor of Philosophy of Loughborough University

October 1998

CK.C.McKenzie Mills, 1998 


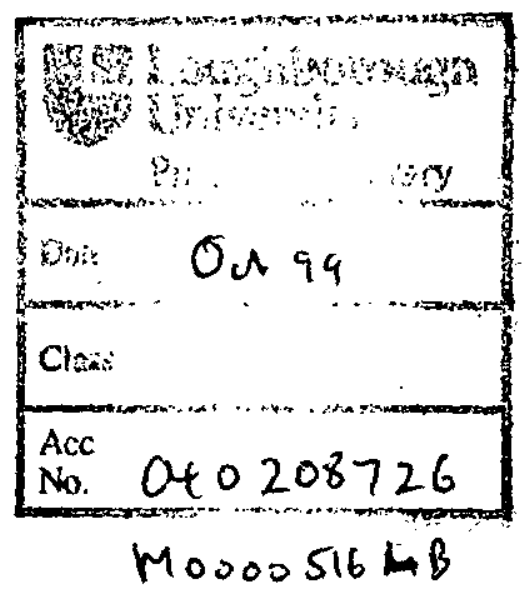




\begin{abstract}
Improving communication between people and computer applications is a major concern of Human Computer Interaction. One possible way of improving such communication is to capitalise on the way human beings use speech and gesture in a complementary manner, exploiting the redundancy of information between the input modes of a system. Redundant data input entered into a computer system through multiple modes, provides considerable scope for the resolution of error and ambiguity in the input message.
\end{abstract}

This thesis presents an investigation of redundant information between modes, through the implementation of a multimodal input system accepting speech and two-dimensional gesture input. A truth maintained blackboard system is used as part of the implemented two-dimensional gesture recognition system, which also uses rule induction to capture the characteristics and variability of the gesture input. Inexpensive commercial speech software handles the recognition of speech input. A multimodal interpreter performs the integration of information between modes and task knowledge.

The role of redundancy is investigated in an experiment using the multimodal input system, examining multimodal recognition performance under a variety of different redundancy levels between the input modes. Redundancy is examined from the viewpoint of computer integration of modes. This is in contrast to the examination of redundancy within the human integration of modes. 
The research presents novel empirical results:

- Multimodal recognition rates achieved from the experiments are statistically significantly higher than either speech or gesture rates alone.

- For simple tasks, multimodal recognition rates increase as the level of redundancy increases. The effect is less noticeable in more complex tasks with higher ambiguous information content.

- The results show that the presence of redundancy reinforces meaning and resolves ambiguity significantly between the modes.

The success of the multimodal integrator is attributed to the weighted comparison algorithm used, and to the presence of task and system knowledge used in the integration process. In conclusion, future multimodal input systems should be designed with redundant data input between modes, integrated in a simple, weighted approach, with careful handling of ambiguous input. 


\section{Acknowledgements}

I would like to thank and acknowledge the following people.

My family: Mum, Dad, and sis-face Marie, for their support. I needed lots of cheering up along the way.

My supervisor, Prof. Jim Alty, for reminding me of the bigger picture when I kept forgetting, and guiding me through. My director of research, Dr. Chris Hinde, for cups of coffee and support. And thanks to Mr Eddie Grant, without whom I wouldn't have started.

Thanks have to go to all of my friends and colleagues in the department who gave me work, support, amusement, money, smiles, coffee, advice, hugs, kisses, curries, and most importantly chocolate: Dr Richard Butterworth, Mrs Marina Carter, Mr Martin Hamilton, Mr Pete Hornsby, Dr Ghada Kadoda, Dr Andrew Kane, Dr Jon Knight, Dr. Colin Machin, Mr Tunu Miah, Dr Kingsley Pang, Mr Karl Perkins, Mr Manu Uniyal.

I also gratefully acknowledge the use of code and systems of Dr. Chris Hinde, and acknowledge the use of code, help, time and support of Mr Karl Perkins. 


\section{Outline of Contents}

Section 1: Introduction to the topic

CHAPTER 1: INTRODUCTION 1

CHAPTER 2: REVIEW OF MULTIMODAL INTERACTION. . .8

Section 2: Initial Work

CHAPTER 3: FOCUSING ON INPUT .56

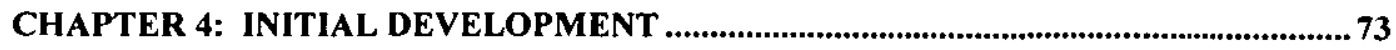

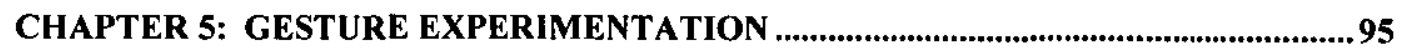

\section{Section 3: Multimodal Work}

CHAPTER 6: THE DESIGN OF THE MULTIMODAL INPUT SYSTEM 125

CHAPTER 7: MULTIMODAL EXPERIMENTAL DESIGN. 144

CHAPTER 8: MULTIMODAL EXPERIMENTAL RESULTS 157

Section 4: Summary \& Conclusions

CHAPTER 9: CONCLUSIONS \& FUTURE WORK .203

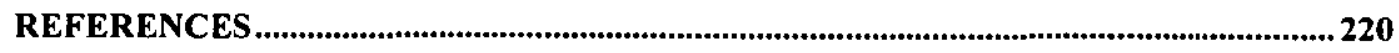

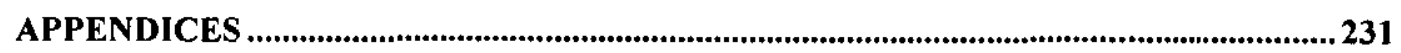

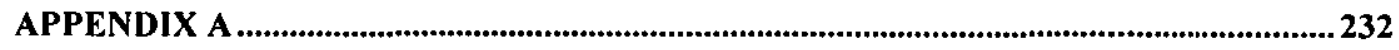

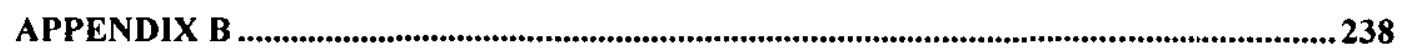

APPENDIX C 


\section{Contents}

\section{Section 1: Introduction}

\section{CHAPTER 1}

1 INTRODUCTION

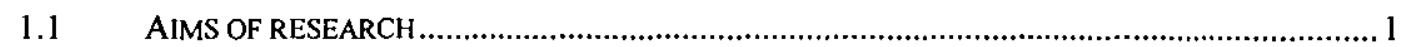

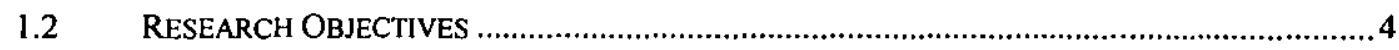

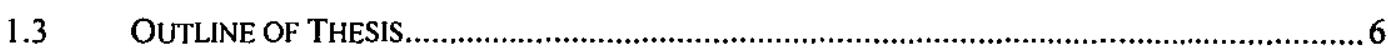

\section{CHAPTER 2}

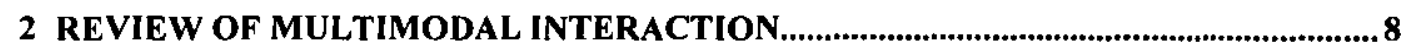

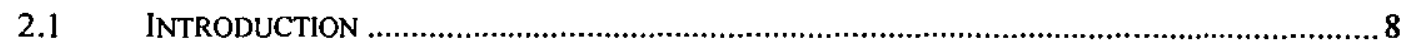

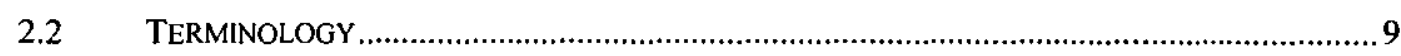

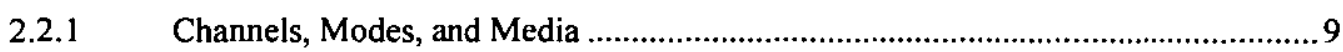

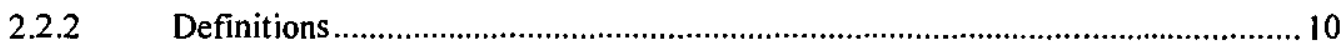

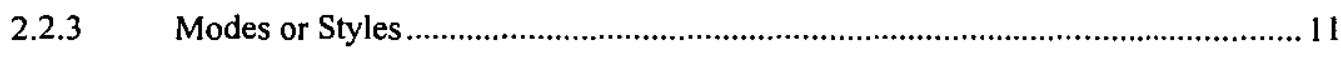

2.2.4 Definition of Redundancy............................................................................. 13

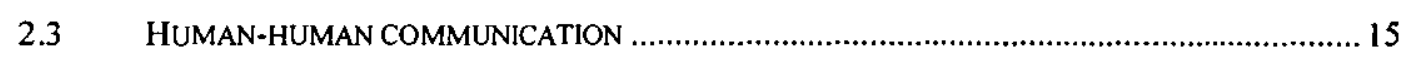

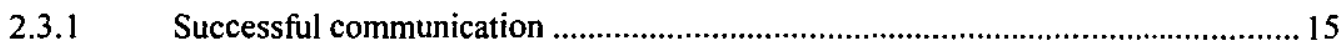

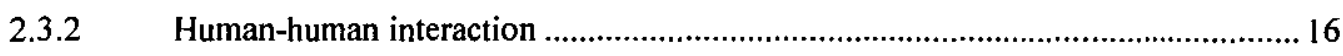

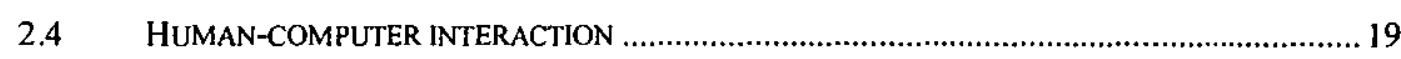

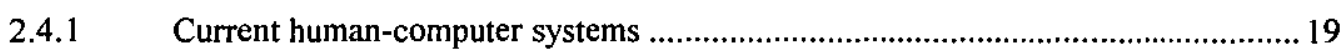

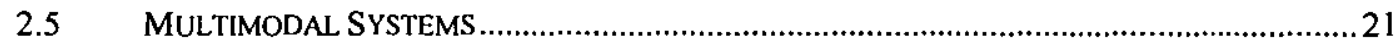

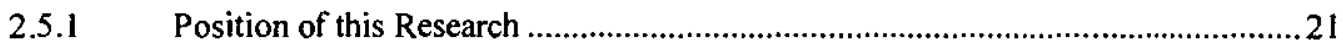

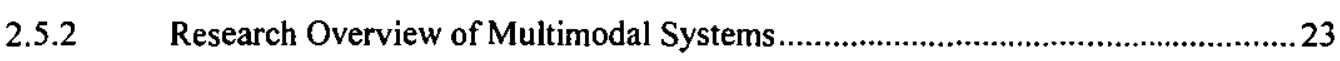

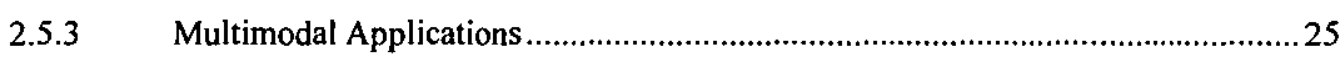

2.5.3.1 Early multimodal input systems ..........................................................................26

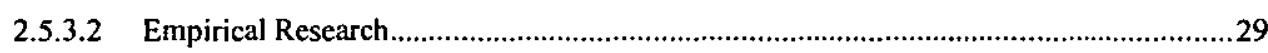

2.5.3.3 Exploratory Research ........................................................................................

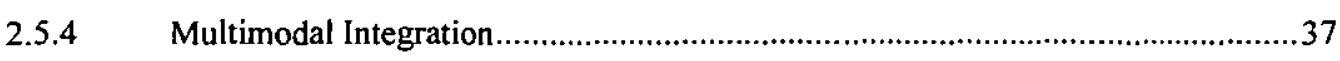

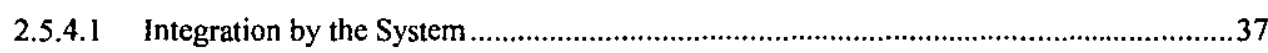

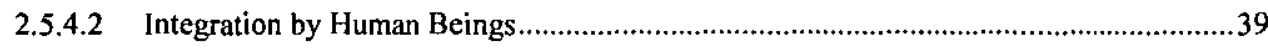

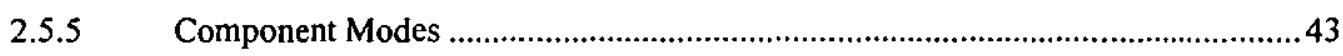

2.5.5.1 Gesture Recognition .....................................................................................4

2.5.5.2 Speech Recognition and Conversational Systems .....................................................48 


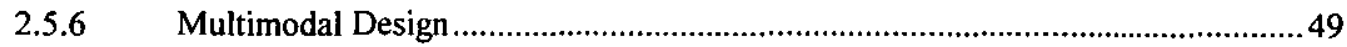

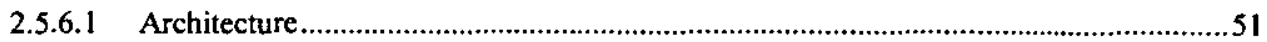

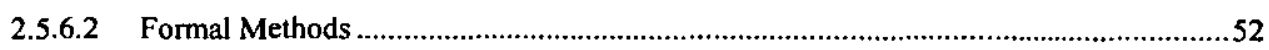

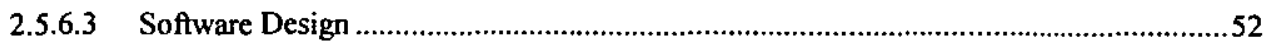

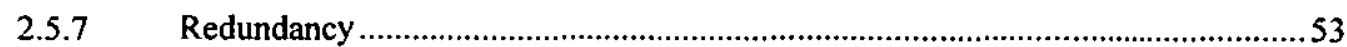

\section{Section 2: Initial Work}

\section{CHAPTER 3}

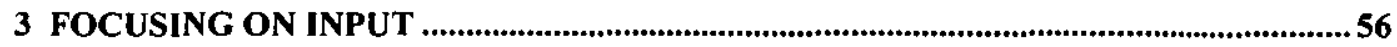

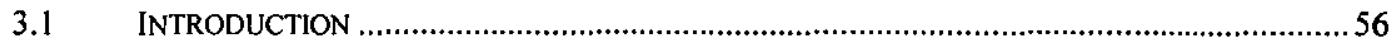

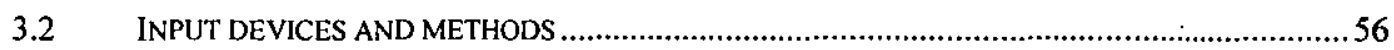

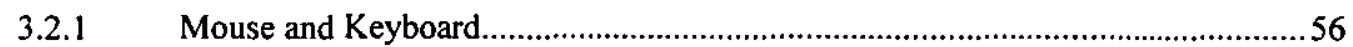

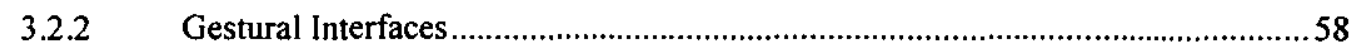

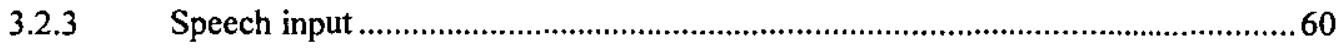

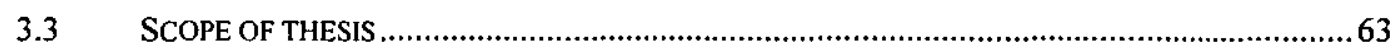

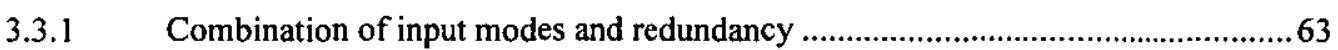

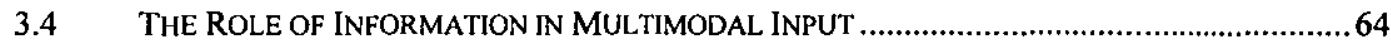

3.4.1 Redundant and Complementary Information........................................................64

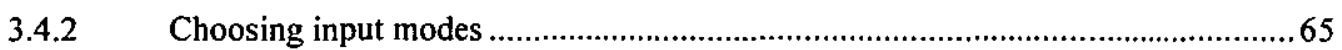

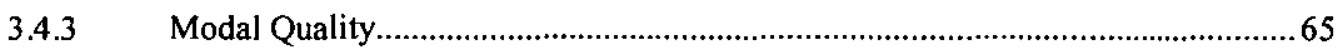

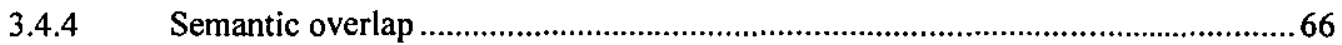

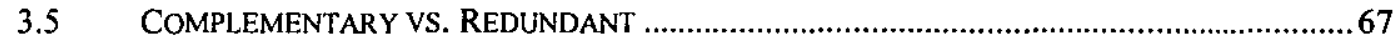

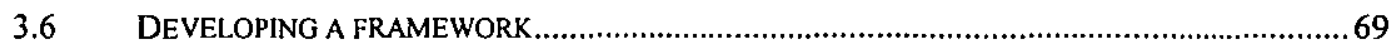

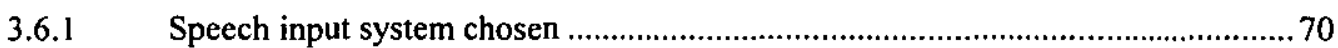

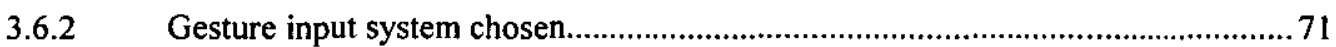

\section{CHAPTER 4}

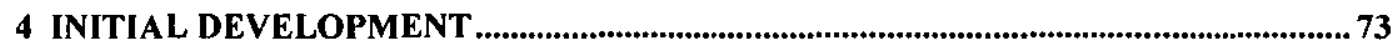

$4.1 \quad$ INTRODUCTION

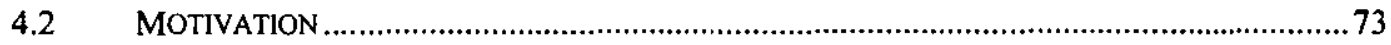

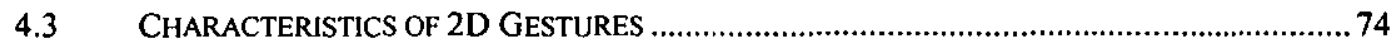

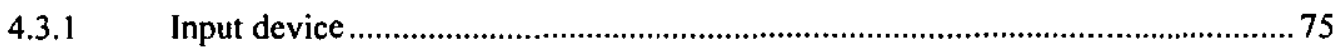

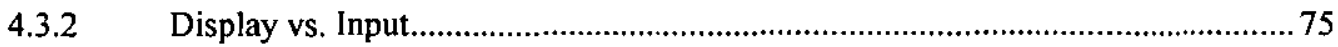

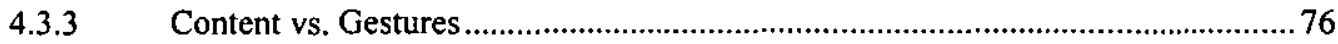

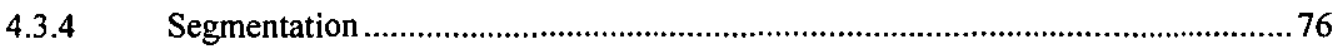

4.3.5 Command Execution ...............................................................................

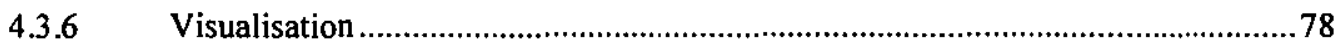




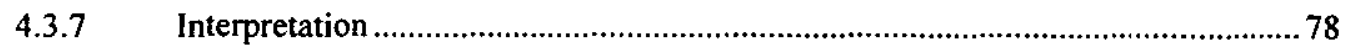

4.4 GESTURE RECOGNITION SYSTEM DEVELOPMENT ………............................................78

4.4.1 Initial gesture recognition system ..................................................................... 79

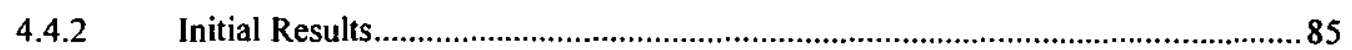

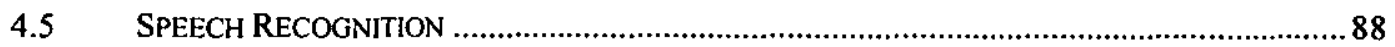

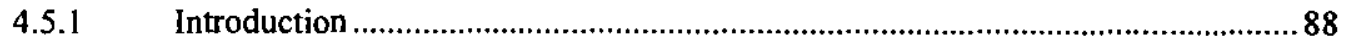

4.5.2 Developing Speech Aware Applications ……......................................................... 88

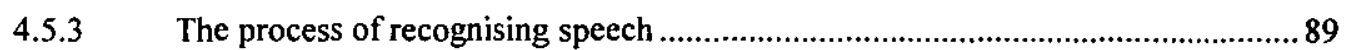

4.5.4 Speech input system developed ......................................................................

\section{CHAPTER 5}

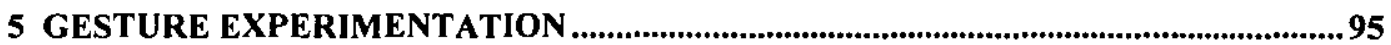

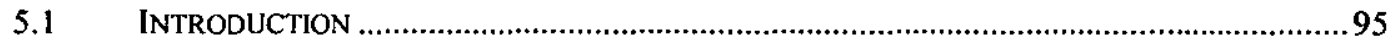

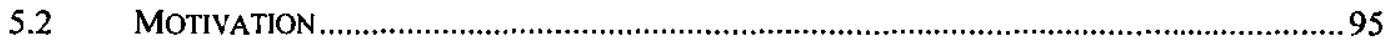

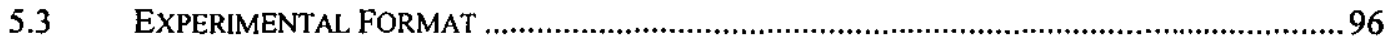

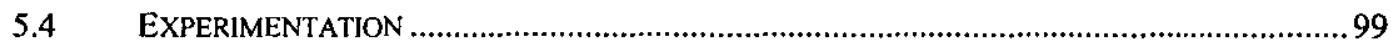

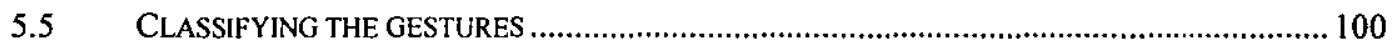

5.5.1 Brief description of Rule Induction .................................................................. 102

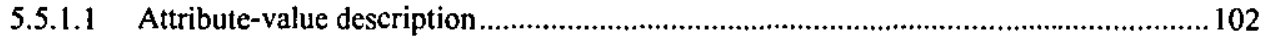

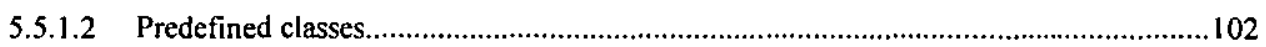

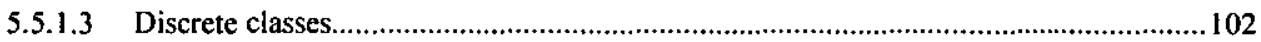

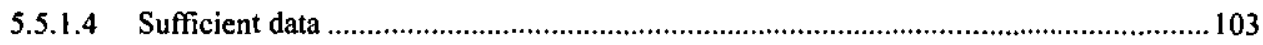

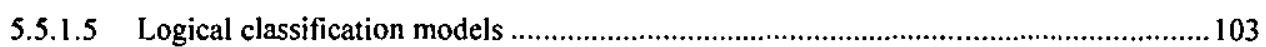

5.5.2 Using rule induction in gesture recognition ...................................................... 103

5.5.3 Preliminary results .......................................................................................... 104

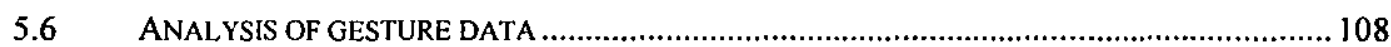

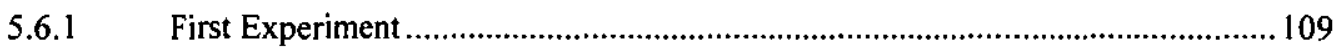

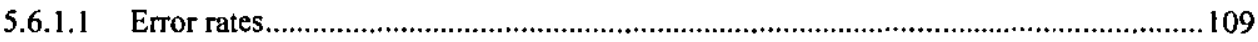

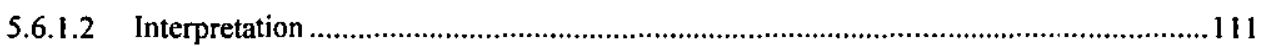

5.6.2 Second Experiment ..................................................................................... 112

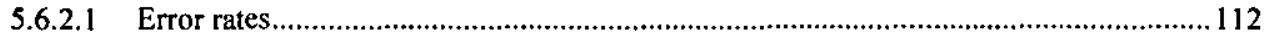

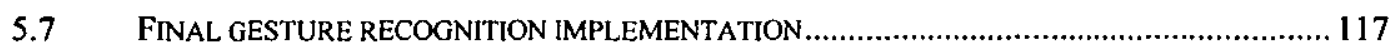

5.7.1 Refining the gesture system ........................................................................... 117

5.7.2 Truth maintenance and biackboard system.........................................................118

5.7.2.1 Properties of Truth Maintenance Systems...............................................................119

5.7.2.2 Relevance to gesture recognition........................................................................... 120

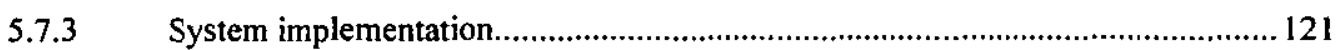

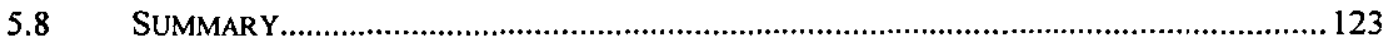




\section{Section 3: Multimodal Work}

\section{CHAPTER 6}

6 THE DESIGN OF THE MULTIMODAL INPUT SYSTEM .......................................... 125

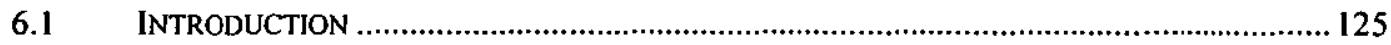

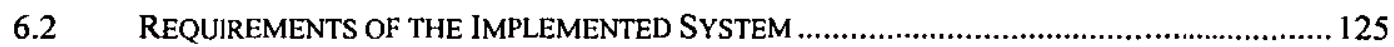

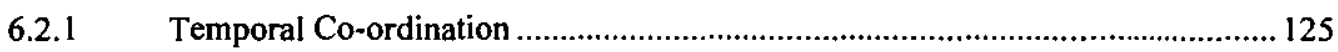

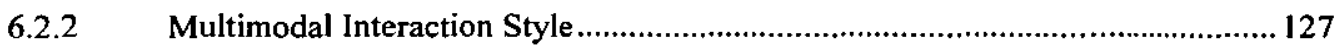

6.2.3 Information situations arising from fusion........................................................ 128

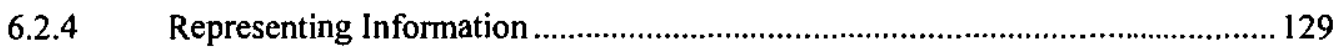

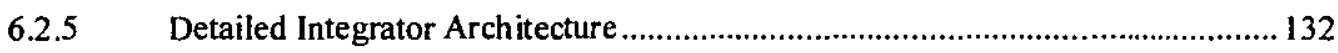

6.2.5.1 Discrete speech recognition system......................................................................132

6.2.5.2 Touch \& Gesture recognition system ...................................................................... 138

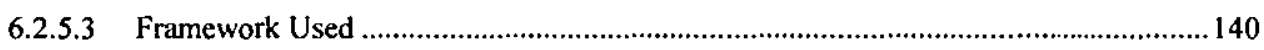

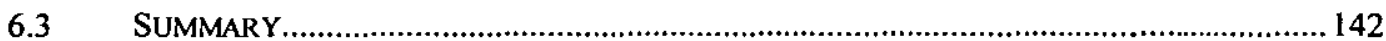

\section{CHAPTER 7}

7 MULTIMODAL EXPERIMENTAL DESIGN ……................................................................ 144

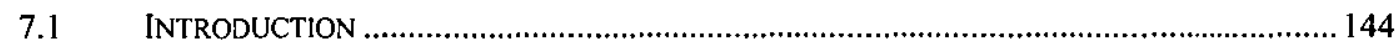

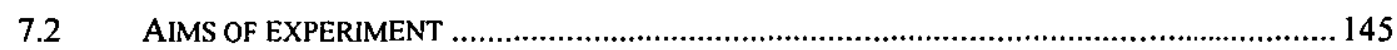

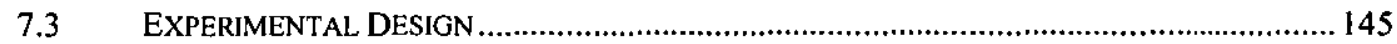

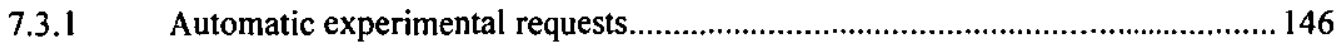

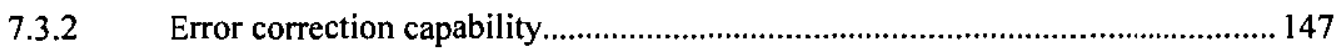

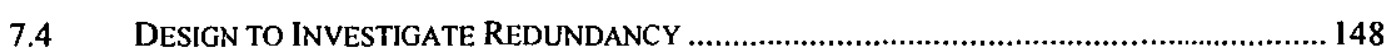

7.4.1 Calculating recognition rates ......................................................................... 150

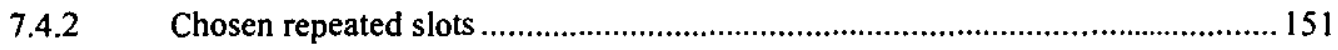

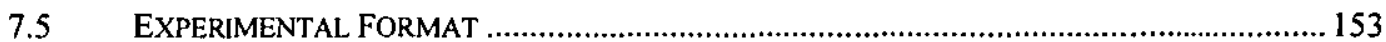

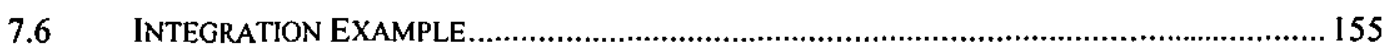

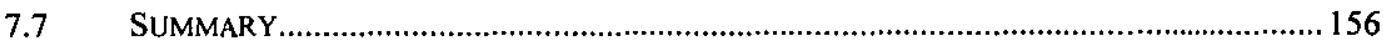

\section{CHAPTER 8}

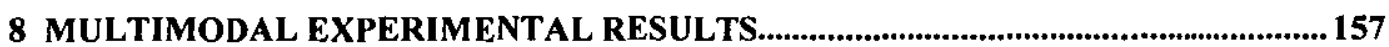

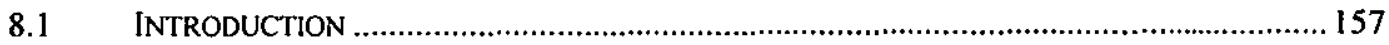

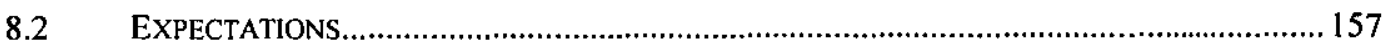

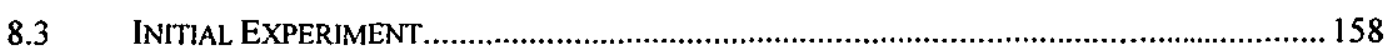

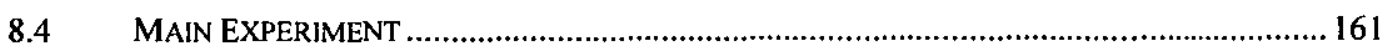

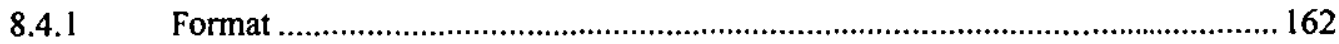

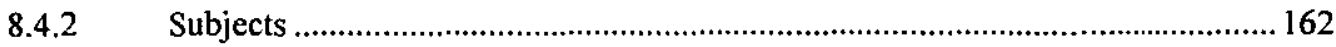




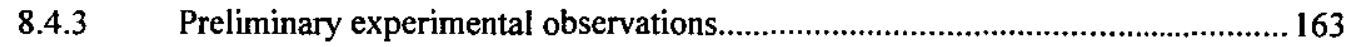

8.4.3.1 Errors made by subjects during interaction ................................................................. 163

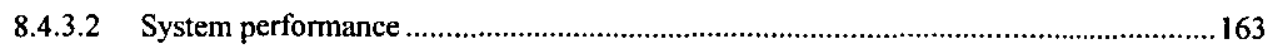

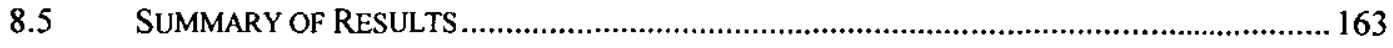

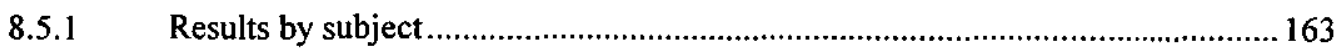

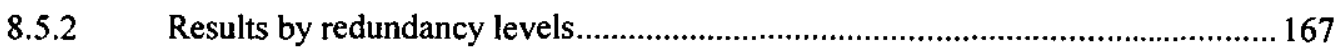

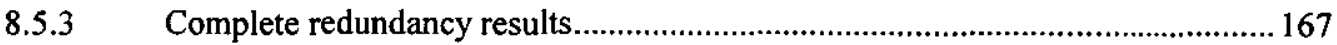

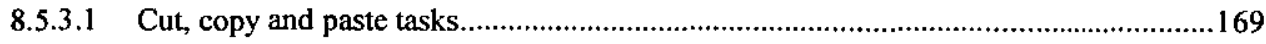

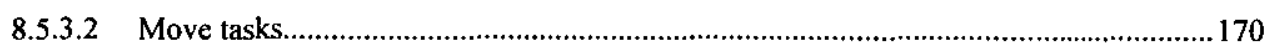

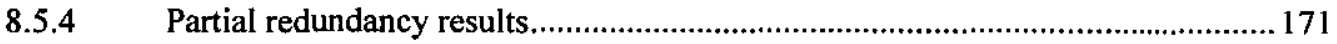

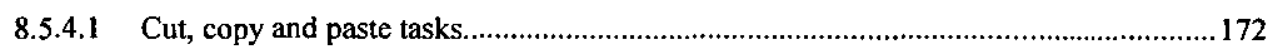

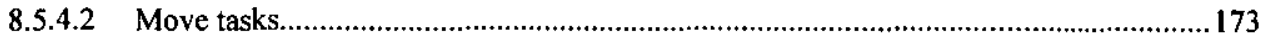

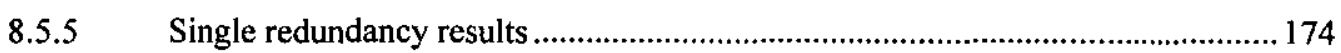

8.5.5.1 Cut, copy and paste tasks.................................................................................. 174

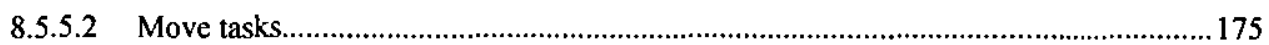

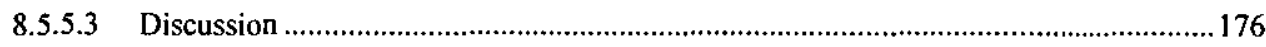

8.6 ANALYSIS OF MULTIMODAL SCORES AND REDUNDANCY …....................................... 177

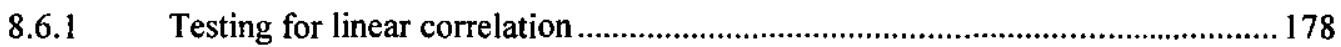

8.6.2 Investigating relationships between redundancy and recognition rates ................180

8.6.2.1 Five main redundancy levels ................................................................................ 180

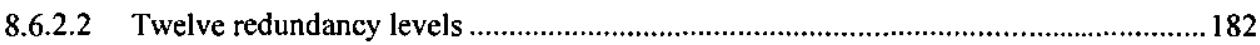

8.6.3 Confirmation of Higher Multimodal Rates ........................................................ 187

8.6.4 Optimum Redundancy level ......................................................................... 189

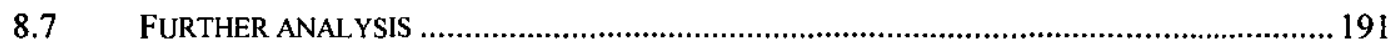

8.7.1 Comparison of primitives recognition over modes............................................. 191

8.7.2 Reinforcement and resolution of meaning with redundancy................................. 197

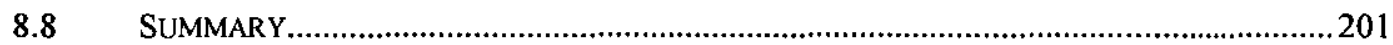

\section{Section 4: Summary \& Conclusions}

\section{CHAPTER 9}

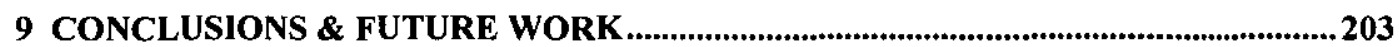

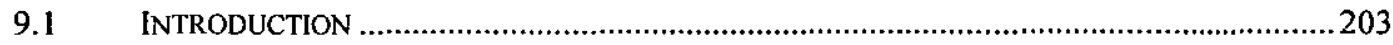

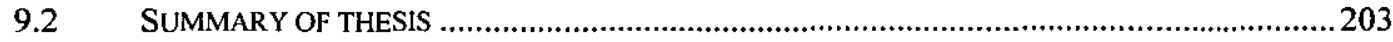

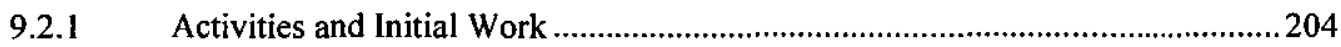

9.2.2 Two-dimensional gesture recognition system ......................................................204

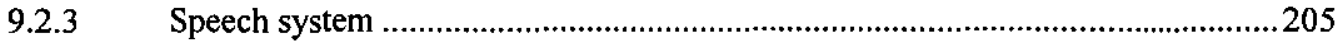

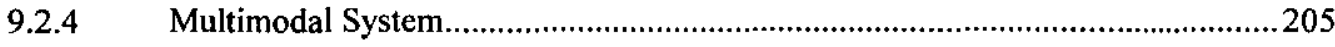

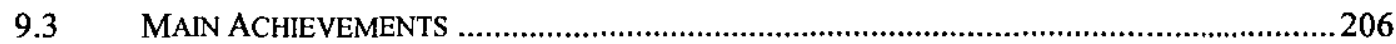




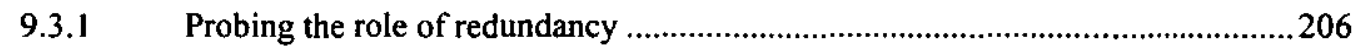

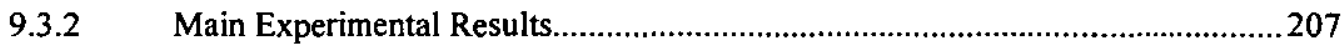

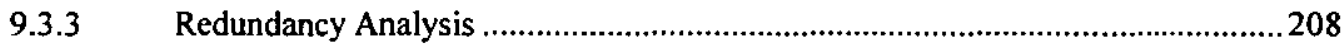

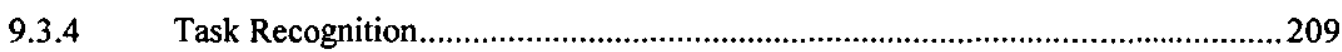

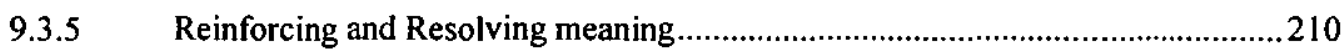

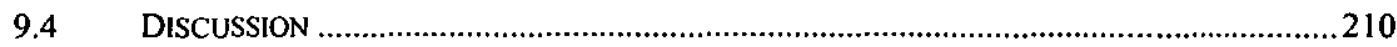

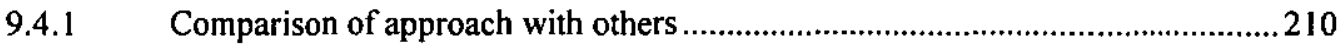

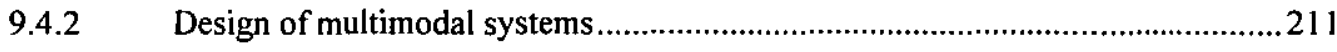

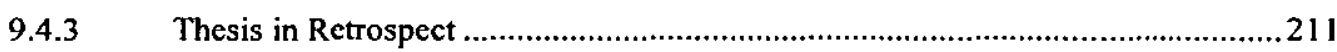

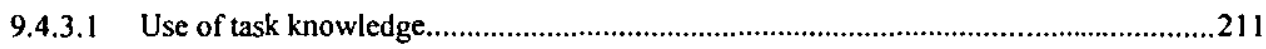

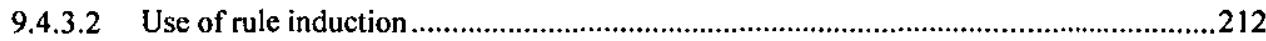

9.4.3.3 Use of truth maintained blackboard system.............................................................213

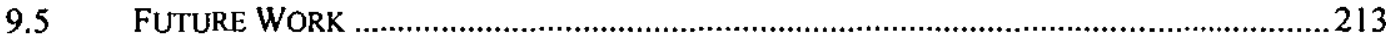

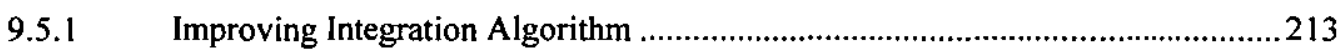

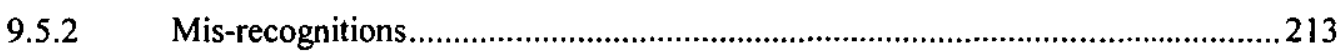

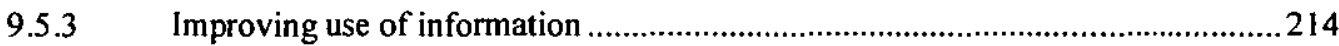

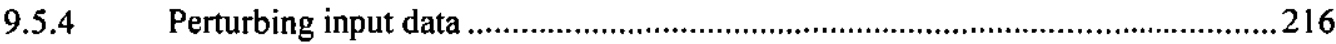

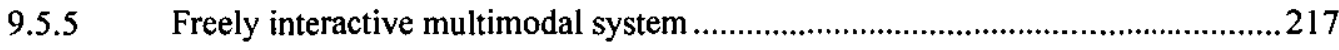

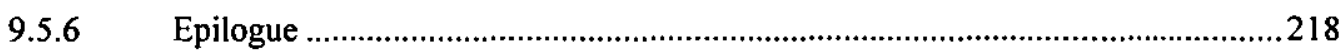

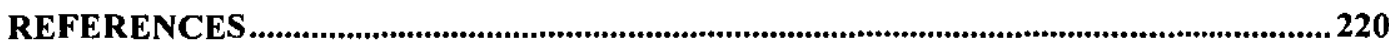

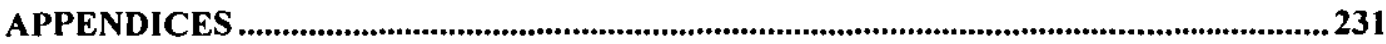

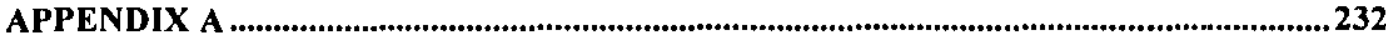

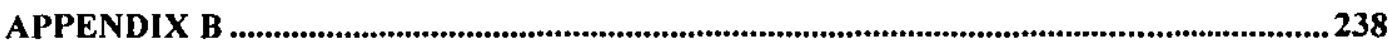

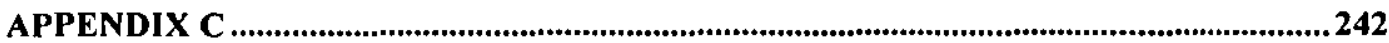


Section 1

\section{Introduction to the Topic}




\section{Chapter 1}

\section{Introduction}

\subsection{Aims of research}

In human-to-human interaction, multiple communication modes such as speech, gestures and body movements are frequently used. By mode, we mean a unique communication modality and interaction style which defines the type of communication channel used to impart information and the manner that the communication is performed by. Thus, speech and gesture modes differ in the method and style of interaction (spoken versus haptic input channels). Generally, humans are successful at communicating their intentions, actions and feelings to one another, because they use a rich variety of modes in combination.

The communicative power of speech, for example, is often enhanced through the use of gestures. Such gestures involve an extensive number of body movements (movement of eyes, limbs; body language, etc.). Frequently, channel content is complementary; that is, the channels provide different information (Oviatt et al, 1997). However, they also can contain redundant information. Human beings, 
therefore, constantly 'send' a mixture of complementary and redundant information and, as a result, achieve a high success rate in communicating their intentions to one another. Redundancy is particularly important where one or other of the channels become indistinct or noisy (Cherry, 1966). In contrast to human-human communication, current computer input techniques are almost never used in parallel and are rarely redundant.

Current human-computer interaction approaches use a number of modes to effect communication (input and output). Whilst the modes for output communication are a reasonable match to those used by human beings, current input modes are more limited. The standard input methods used, such as text input via the keyboard and location information from a mouse, do not provide the optimum communication environment. In addition to providing a more natural interaction, the use of multiple modes also enhances the input recognition task (Caloini \& Tosolini, 1994; Cassell et al, 1994; Edwards, 1992; Oviatt et al, 1997, Oviatt \& Olsen, 1994; Vo \& Wood, 1996). However, flexible input combinations more suited to human capabilities (for example, speech and gesture modes) are not yet a standard desktop combination for human-computer interaction.

The concept of multiple channel human-computer interaction is not new. As far back as 1980 the 'Put-that-there' study at MIT (Bolt, 1984; Bolt, 1987) involved a speech and three-dimensional gesture input system. However, speech and gesture input have yet to be fully integrated into popular interfaces. Currently we only really use the mouse and keyboard, which are limited as they do not exploit all of the communication skills that human beings possess.

Recently, efforts have been made to improve single mode input methods. Research in voice recognition has advanced sufficiently to provide continuous speech recognition software (Fishman \& Padilla, 1997), whilst discrete voice recognition software had been available for several years. Gesture recognition, covering two and three dimensions, ranging from two dimensional gesturing on screen to whole body movement is still in its developmental infancy (see Wachsmuth \& Frohlich, 1997 for detailed current research status). 
Research into the integration and combination of multiple modes is fairly new and little progress has been made in making the concept commonly available on our desktops. This is despite a large variety of initial studies reported between 1993 and 1998 (Ando et al, 1994; Ando et al, 1995; Bordegoni, 1994; Davis \& Shah, 1996; Duchnowski et al, 1994; Vo \& Wood, 1996).

In multimodal systems, where input can be achieved through multiple channels, there are multiple and parallel sources of information. Initial studies into the integration of input modes have focused on the combination of modes, and have shown that multimodal recognition levels were higher than the single mode rates (Ando et al, 1995; Ando et al, 1994; Koons et al, 1993; Nakagawa et al, 1995; Vo \& Waibel, 1993b), even when one mode was performing poorly. This benefit was attributed to the presence of redundant information across the modes, which was called upon at times of ambiguity, to resolve an outcome (Akamatsu \& Sato, 1994; Vetere, 1997; Vetere et al, 1997). This can be exploited for error resolution (Oviatt, 1996; Oviatt \& VanGent, 1996), as well as the resolution of ambiguities and contradictions (Nakagawa et al, 1995; Vo \& Waibel, 1993a).

Although research argues that multiple input modes have benefits over a single input mode, there is little empirical evidence of the benefits or why they occur. The majority of recent research work has insufficient analysis, results or considerations of how and why multiple modes can assist in the resolution of errors and ambiguities. One obvious area of study is information redundancy between input modes. Such a study could give insights into the advantages of multiple input modes and provide more rigorous analyses.

To gain insight, and to be able to progress in the design and creation of multimodal interfaces, we need to have an understanding of the components and how they fit together. Obviously, the initial inspiration for multimodal interfaces comes from imitating human beings. Human beings use their communication skills in parallel and their understanding of rich communication activity is based on their knowledge (of surroundings, what you see, what you hear). More pertinently, when human beings communicate (or give input) they may transmit 
the same information in more than one way, such as pointing to an object and speaking about it at the same time. Thus some of the information might be redundant at the end of the conversation if the receiver clearly understood from the speech alone which object was under discussion. However, if the receiver did not hear properly then the pointing gesture could resolve ambiguity and would not then be redundant.

The initial aim of this research was to create a simple, inexpensive multiple input system in order to investigate the role played by redundancy of information across input modes. The chosen input modes were speech, and two dimensional gesture.

The stress is laid on simple and inexpensive input modes, as we wished to investigate the key factor of multiple input systems, redundancy of information between modes, without the best possible quality of recognition of input. The success of the system would come through the exploitation of redundancy and additional knowledge in the system, mimicking how we, as humans, constantly communicate and interpret in our surroundings.

\subsection{Research Objectives}

This research presents an investigation into the role of redundancy between multiple input modes. Domain and task knowledge are used to resolve the human-computer interaction ambiguities, using the input modes of speech and two-dimensional gesture. 
The main research objectives are:

- To probe the role of redundancy in multi-modal interfaces

- To determine levels of recognition success at which redundancy has some or best effect

- To analyse the relationship between redundancy and multimodal recognition rates

- To investigate if multimodal recognition rates are higher than separate unimodal rates. If this is the case, where does the gain in recognition success come from?

- To investigate how often redundancy of information reinforces meaning when two input modes agree

- To investigate how often redundancy of information resolves meaning when two input modes disagree

As mentioned earlier, complex three-dimensional gesture recognition is being developed through many research efforts (Wachsmuth \& Frohlich, 1997). Some work concentrates on hand movement, gestures and sign languages while other work investigates whole body movements. Some of the most current and powerful computer technology can be used to 'solve' this problem of optimal performance when combining multiple input modes such as speech, touch and gesture. Neural networks are used for such complex gesture recognition and for speech recognition (continuous and discrete). It would seem reasonable to argue that use of the most up-to-date hardware and software could allow 'the best' recognition to occur or allow the best chance of optimal recognition.

However, we aim to show that without use of the strongest computational power (fastest processing, optimal hardware and software) a simple multimodal system can become a viable option. This comes through the exploitation of the critical factors that create the benefits of a multimodal system, namely, the reinforcement of meaning and resolution of ambiguities through redundancy and use of relevant prior knowledge. Indeed, we will go further. We aim to show that a simple multimodal approach will work effectively. 


\subsection{Outline of Thesis}

The thesis is divided into four main sections: Introduction (Chapters 1 and 2), Initial Work (Chapters 3,4 and 5), Multimodal Work (Chapters 6, 7 and 8) and Summary \& Conclusions (Chapter 9, References and Appendices).

The current chapter, Chapter 1, introduces the general topic of the research and provides the outline of the thesis. Chapter 2 discusses the properties of humanto-human communication which can be exploited in human-computer interaction. The chapter also introduces terminology, including modes and media of interaction. This is followed by a literature review of past and current research in the area.

Chapter 3 reduces the scope from the previous chapter, focusing on the properties of input only. A review of current input methods is presented, showing the need for exploitation of more natural methods of input to computers. The use of multiple input modes is discussed, with a section considering the possible relationships that may occur between multiple input modes. In particular the possibility of redundant relationships is examined. This chapter ends with a brief discussion of methods of experimentation, and of the speech and gesture systems used in the research.

Chapter 4 presents the initial developments for the multimodal input system. The requirements for the two dimensional gesture recognition system are discussed, and the design of the system is presented. The properties of two-dimensional gestures are discussed, and the preliminary results gained during the development of the system are also explained. The requirements for the speech recognition system are also discussed.

Chapter 5 presents the experimentation performed using the developed gesture recognition system. The use of rule induction on the data gained from the experiments is presented, which generated production rules. A truth maintained blackboard system (provided by Hinde et al, 1989) using the production rules to 
form the knowledge bases of the system is introduced. This system was used in the final gesture recognition system implementation, as part of the final multimodal input system.

Chapter 6 introduces the multimodal integrator, detailing the processing steps required, and the structure of the integrator used in the multiple input application. The requirements of the multimodal integrator are discussed, followed by the chosen properties of the developed structure.

Chapter 7 describes the multimodal experimental design, using the multimodal integrator introduced in Chapter 6. The aims and expectations of the experiment are also discussed. Chapter 8 examines the result of the multimodal experiments, and presents the novel empirical results about redundancy between modes.

Chapter 9 presents conclusions and details future work which could be conducted in this area. The references and appendices follow this final chapter. The appendices contain data to complement graphs displayed in chapters throughout the thesis. Where appropriate guide sheets used during experiments are also shown. 


\section{Chapter 2}

\section{Review of Multimodal}

\section{Interaction}

\subsection{Introduction}

In this chapter the terminology used in the thesis is introduced and the main properties of both human-to-human communication, and human-computer interaction are discussed.

Human beings are skilled, and successful, at conversing with each other. They are able to manage complex and varied styles of communication, connection and interaction (Cherry, 1966). This contrasts with the ways in which human beings interact with computers, which, in general, are less successful than human-human interaction techniques. An examination of the styles and methods of humanhuman communication, therefore, may provide pointers as to why interaction with computers is relatively impoverished. 
The discussion focuses on the nature of communication between human beings and computers, and in particular the interaction between them. The benefits of mimicking human-human communication in human-computer interaction are examined, specifically, human exploitation of many methods and channels of communication in parallel. This examination suggests a means of improving the input recognition task for human-computer interaction, by using additional information from parallel channels, to assist with the recognition process particularly in times of ambiguity.

\subsection{Terminology}

\subsubsection{Channels, Modes, and Media}

In discussions about multimedia and multimodal interfaces many terms are used which are often poorly defined - channel, medium, mode, sense, style. There is no agreed set of definitions for these terms (Alty et al, 1993).

The word medium is defined in the dictionary as:

'something through which an effect is produced (e.g. Air is the medium through which sound is carried to a distance): means (e.g. TV is a powerful advertising medium)'

(Chambers Dictionary)

There are at least two different uses of the term "medium" in computing. A medium can be a storage mechanism for information (for example a hard disk, a floppy disk or a magnetic tape). Alternatively, the term is used to describe a communication technology (or ways of representing and communicating input and output information). It is in this latter sense that the term medium will be used in this thesis." Computer professionals talk about "output media" such as printed text, graphics, video, speech and sound, or "input media" such as the keyboard, the mouse or function keys, but we need more formal definitions. 


\subsubsection{Definitions}

A Channel is a human sense used to receive or send a message. There can be Auditory channels, Visual channels, Haptic channels, Taste channels and Olfactory channels. All communications between human beings and computers require at least one reception or receiving channel, though communication can involve more than one channel simultaneously.

Our viewpoint of channel is always from the human perspective, that is, we are concerned with Sending channels for INPUT to computing devices, and Receiving Channels for OUTPUT.

A Transmission Mechanism is used to move a message from one place to another. Examples include radio waves, light waves, sound waves, electric currents etc. Usually, such mechanisms have Transducers at each end to convert from human senses into the transmission mechanism and vice-versa. For this reason, the transmission mechanism is not really of any importance for our purposes (except that its constraints may limit the expressiveness of the medium of communication carried).

Messages are communicated along Sending or Receiving channels. The term, Medium, refers to the way in which the messages are built, constructed, and how they are used. The properties of the Medium define the complete set of possible messages that can be transmitted, but not the content itself.

The properties which define a Medium are its Lexicon, Syntax, and Pragmatics (Mayes, 1992). The basic building blocks used in messages make up the Lexicon, the ways in which these building blocks are put together are called the Syntax, and the ways in which messages are used, are termed Pragmatics. Because the human sensory channel used is so basic to the nature of possible building blocks employed, it is also included in the definition of medium.

In theory, one need not include the channel in the definition of medium. However, without its inclusion the definitions for the building blocks-and syntax 
often become complex. Consider, for example, the two distinct media "written language" and "spoken language". Precise definitions for the building blocks for the two media could be developed, since there are subtle differences in the way sentences are constructed and how words are used in the two media. This would be unnecessarily complex however. It is much simpler to include the auditory and visual channels in the definition, so that the distinctions become obvious. Using the sensory channel also conforms to Frohlich's scheme (Frohlich, 1991), called 'Design Space', which classifies media into Audio, Visual and Haptic.

The following table gives examples of media and their associated building blocks.

\begin{tabular}{|l|l|l|l|l|}
\hline Medtum & Builuing blocks & Syntax & Practical use & Clianuel \\
\hline Keyboard input & $\begin{array}{l}\text { Characters, } \\
\text { Punctuation, } \\
\text { Numerics }\end{array}$ & Grammar & $\begin{array}{l}\text { Commands, } \\
\text { Carriage return, } \\
\text { Line feed, Fonts, } \\
\text { Layout }\end{array}$ & Haptic IN \\
\hline Speech input & $\begin{array}{l}\text { Words, Silence, } \\
\text { Phrasing }\end{array}$ & $\begin{array}{l}\text { Gonversation start, } \\
\text { Conversation end }\end{array}$ & Audio IN \\
\hline Gesture input & $\begin{array}{l}\text { Gesture strokes, } \\
\text { pauses }\end{array}$ & $\begin{array}{l}\text { Gesture language } \\
\text { grammar }\end{array}$ & $\begin{array}{l}\text { Gesture start, } \\
\text { gesture end }\end{array}$ & Haptic IN \\
\hline
\end{tabular}

Table 1: Examples of media and their characteristics

It can now be seen why a medium is more than a transmission mechanism. Radio waves are a transmission mechanism, but they can support a number of media. One example would be the medium of Morse Code. Another example would be the medium of Speech (the radio telephone) and a third example would be the medium of Music Broadcasts. All of these use the auditory channel for reception, and all can use radio waves for transmission. We can, however, change the mechanism of transmission without changing the medium. All of these could equally be transmitted down a copper wire. We cannot, however, change the reception or sending channel, without changing the medium. Written words are not the same medium as Spoken words.

\subsubsection{Modes or Styles}

In addition to channels and media, research often refers to the 'mode' of communication. The term can be used in two ways. 
- To describe the channel used (audio, visual, tactile)

- To describe the 'style' of interaction between human beings and computers

It is clear that the latter interpretation is more embracing than either medium or channel. It refers to the nature of the interaction being engaged in. In this sense a mode (or style) occurs over time and involves both participants. It might be termed the 'style' of interaction.

Frohlich (1991) defines only two modes - Language and Action. In his view, a mode defines a relationship between user actions and effects of these actions on a system. Bernsen defines input modality (Bernsen, 1995) similar to our definition of medium:

'..input modalities are forms of information representation communicated to the system (or computer) by its users'

Nigay and Coutaz (1993) define modality as a reference to the "..type of communication channel used to convey the information. It also covers the way an idea is expressed or perceived, or the manner an action is performed.' They also define Mode as a 'state that determines the way information is interpreted to extract or convey meaning.'

These mode definitions therefore seem to be connected with the manner in which an interaction is performed. An example would be the difference between direct manipulation and a language (conversational) approach. In both cases the description refers to the nature of the interaction itself rather than the disembodied (separate) input and output messages.

Since the concept of mode is one of interaction, it is not surprising that most of the definitions of mode do not make a distinction between input modes and output modes. Mayes (1992) defines mode in the context of 'interaction' without specifying separate input or output aspects, and Nigay and Coutaz (1993) define the extraction and conveyance of meaning in a mode, which relates to both input and output. Hutchins (1989) also demonstrates the difficulty in separating input 
and output in any definition of modes. In his work on metaphors in design, he defines 'mode of interaction' metaphors as metaphors which

'..organise understandings about the nature of the interaction with the computer. Mode of interaction metaphors concern the relationship between the user and the computer without regard for the particular task the user is attempting to accomplish via the computer.' (Hutchins, 1989)

He goes on to say 'The choice of metaphor... determines what sort of thing the user thinks the computer is. Is it a conversational partner? An environment for action? A tool box and materials shed?' (Hutchins, 1989). Hutchins is therefore proposing that how the user is interacting, and what the user is manipulating to perform the interaction, affects the mode of interaction. Therefore, we may conclude that it is not possible to completely define a mode of interaction without consideration of both input and output, and consideration of the complete interaction process.

In this thesis we are more concerned with the relationships involved in the joint use of input channels than with the interaction styles. Our definition of mode therefore is that of input modality - the senses used to communicate.

\subsubsection{Definition of Redundancy}

In the introduction to this thesis, the concept of redundancy was introduced, referring to the possibility of repeated or duplicate information being carried across multiple input modes.

There are several uses of the terms 'redundancy' and 'redundant'. For instance, in some of the work of Oviatt et al (Oviatt, 1995; Oviatt, 1996; Oviatt et al, 1997, Oviatt \& Olsen, 1994; Oviatt \& VanGent, 1996), the redundant use of multiple modes is frequently referred to as being equivalent to the simultaneous use of multiple modes. This is corrected in some later work, where redundancy is referred to as the provision of duplicate information. 
Redundancy can also refer to the inclusion of extra, related information (not duplicate or repeated but different) which may not always be utilised. A classic example is that of traffic lights (Edwards, 1992), where both colour and position information are supplied, but the position information could, in normal circumstances, be regarded as surplus, or redundant to the explanation of how the traffic lights operate. We associate green with go, red with stop, red and amber with prepare to go, etc. In theory, at least, the position order of lights does not matter.

The fact that these colours always appear (in some combination) in the top, middle and bottom positions, respectively, seems somewhat surplus (or redundant) to an understanding of how traffic lights operate. However, if sets of traffic lights did not have a standard position layout, drivers could be confused. Green might appear on the top light in some traffic signals, the middle light in another set, or the bottom light in a different set of lights. Therefore, the apparently redundant information has an important purpose: it clarifies possible ambiguities, avoiding confusion by providing valuable extra information. Marmollin also notes the effects of colour in multimedia presentations, commenting that the redundant use of colour '..facilitates the pick up and processing of information..' (Marmollin, 1991).

The traffic light example could be described as within-channel redundancy, as the same channel has 'transmitted' different information, exhibiting unimodality. Where more than one mode is involved, between-channel redundancy is possible.

Redundancy is defined as the provision of duplicate (repeated) information which can be used to support information, or resolve ambiguity of information in the same or another channel. 


\subsection{Human-human communication}

\subsubsection{Successful communication}

Human beings use a wide variety of methods and techniques to communicate with each other (Cherry, 1966). Communication can be a face-to-face interaction, or indirect activity (but not necessarily less successful) e.g. written communication, communication from radio, television, film etc. Human beings use various techniques for sending and receiving information, often simultaneously. We speak in conversations, point to the subject of the conversation, and gesture to highlight our speech. Additionally, our body posture, manner of listening to others as they speak, our facial expressions, and eye contact, can all transmit meaning to our conversation partner. (Cherry, 1966)

Human beings achieve successful communication in four main ways:

- They can interpret many sources of information in parallel, e.g. they process voice and gesture together, using either source to assist the other at times of confusion. Gesture is also used for emphasis (McNeill, 1996).

- They have an implicit understanding of the likely errors in different sources of information, and use this understanding to weight the sources e.g. if they mis-hear some parts of an utterance, they can make a best guess about the most likely missing part (Cherry, 1966; Oviatt \& VanGent, 1996)

- They rely heavily on knowledge about the content of likely messages, in context, to assist in disambiguation, e.g. they instinctively use gesture to support speech when ambiguity is possible or when their perception of understanding may be compromised (Cassell, 1994)

- They are able to use interaction to effect repairs to the conversation. When misunderstandings arise, they use interactivity to probe these misunderstandings (Waibel et al, 1997, Vo \& Wood, 1996)

The key factors which are most relevant to this thesis are the first and third properties outlined above - the benefits which arise from several communication techniques operating in parallel, and the application of task knowledge in context. The parallel information from gesture may be distinct from, or equivalent to, the speech, and in either case provides another dimension (either complementary or redundant) to the interaction. The use of several simultaneous (or parallel) 
communication techniques or media in human-computer interaction introduces benefits for both input and output communication.

\subsubsection{Human-human interaction}

Most human-to-human conversations are examples of interaction (Cherry, 1966). The connection and relation between the parties in a conversation, makes it a meaningful and reciprocal exchange of information, rather than a set of unrelated exchanges (of information). Therefore, interaction only occurs when some type of inter-related exchange of ideas, conversations, and representations occur (Cherry, 1966)

Many other forms of human-to-human communication are not interactive. For instance, although, a film director is communicating to his audience through a film, the audience cannot usually respond directly to the film whilst watching it. Therefore, this type of communication is one-sided, as the transmission of information is only occurring in one direction (from the film to the audience). The attention in this thesis will be focused on two-sided communication and interaction.

Consider the following, linear model of communication (from Adler \& Rodman, 1988), shown in Figure 1.

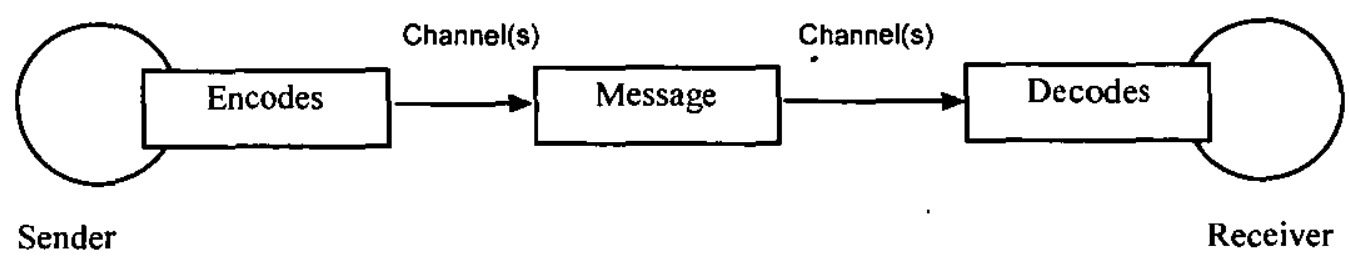

Figure 1: A linear model of human-human communication, taken from (Adler \& Rodman, 1988)

This model defines a sender who begins the process of communication, and who encodes their thoughts into some form of representation or message. This message is encoded by use of specific mechanisms of communication (e.g. 
speech, gesture, written communication etc.), and is then transferred to the receiver. The message must then be decoded by the receiver of the message back into the meaning intended (Adler \& Rodman, 1988).

While this model identifies the basic elements of communicating such as a sender, receiver, and means of communicating (the channels), it omits the concepts of feedback, reaction and inter-connection, that is, it does not include a representation of the interaction itself. Interaction could be represented in the model by the combined (and perhaps simultaneous) sending and receiving of information by the participants, defining them as both senders and receivers at any given time (Adler \& Rodman, 1988; Cherry, 1966; Tubbs \& Moss, 1994).

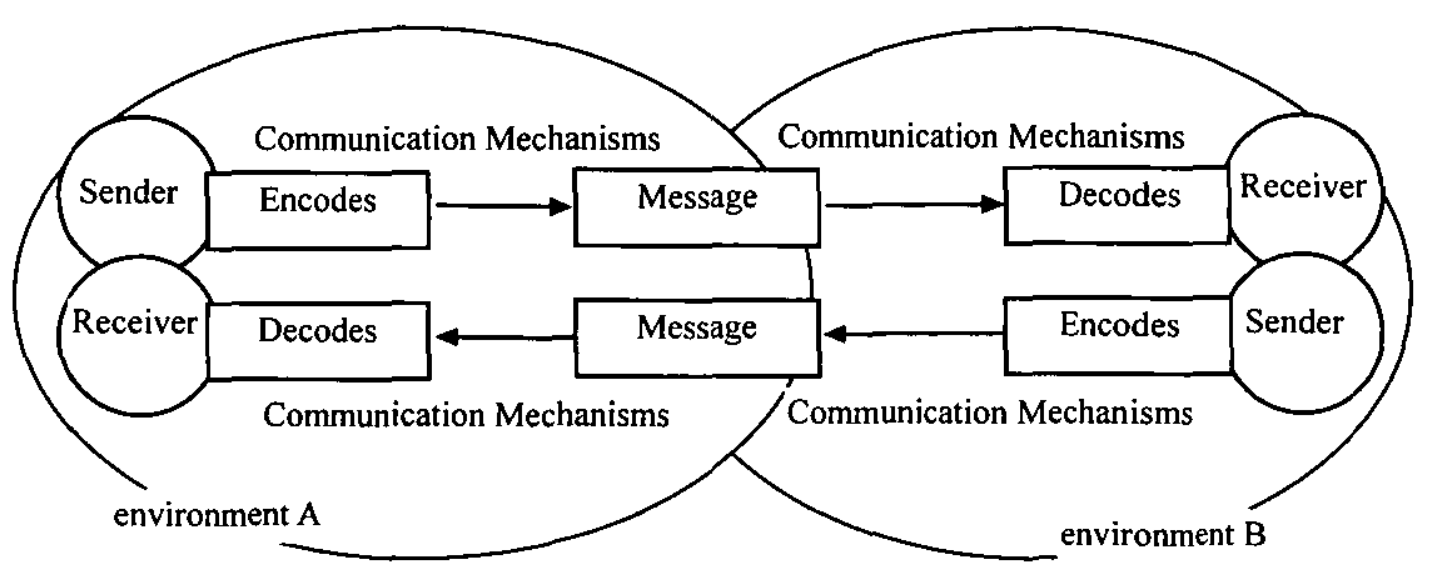

\section{Figure 2: Interactive model of communication, from (Adler \& Rodman,} 1988)

The model shown in Figure 2 demonstrates how the participants in an interaction are constantly sending and receiving communication messages, which may take various forms, and be transmitted through various mechanisms of communication. The environments of the participants also affect the interaction and communication process (where the environment could include the knowledge and experience of that participant which affects their responses). (Tubbs \& Moss, 1994) 
The model also suggests the possibility of the combined use of more than one communication mechanism in parallel. Thus, a message may be encoded in both speech and gesture, and the information contained in both communication mechanisms could have one or more of several different relationships to each other: either distinct (contradictory, emphasising, complementary), or duplicate and repeated. The scope of human communication is shown through the capability of utilising distinct information from several mechanisms. The communication bandwidth is increased by the addition of gestures which complement speech. (Adler \& Rodman, 1988)

Human beings rarely realise how important redundancy is (Adler \& Rodman, 1988). Consider for example, the interaction that takes place at a meeting with several people participating. There may be a blackboard or presentation screen in the room. The people at the meeting may discuss the contents of the board and gesture towards it while discussing something. These people will most probably gesture towards one another while speaking as well. They will use speech, pointing, visual presentation, eye contact, and will also be watching the facial expressions, posture and body language of others. If a tape recording is made of this meeting, however, it will be more difficult to comprehend, because the tape will only contain the verbal and sound content of the meeting, disembodied from all the other contributing communication activity. (Adler \& Rodman, 1988)

Some communication techniques are sophisticated. For example, we can communicate either explicitly or implicitly: explicitly through speech and gesture, or implicitly through glances, body posture, attitude etc. All of these activities can happen separately or in tandem, and taken together, they represent a highly complex process of communication.

In comparison to the wealth of communication techniques that human beings have at their disposal, a computer is a poor communication partner. It is missing many elements that humans take for granted. However, we can almost certainly learn to improve computer-human interaction by observing human-human interaction, and implementing relevant aspects of it. 


\subsection{Human-computer interaction}

\subsubsection{Current human-computer systems}

Human-computer interaction can be compared with our basic model of humanhuman communication (Figure 2, pp 17), where a human being represents one participant of a conversation, and the computer represents the other participant.

When human beings communicate information to a computer system and interpret its actions during interaction, they do so in order to achieve their goals using the system, and must transform their mental representations into input actions at the interface (i.e. digital signals). Thus, human goals and actions have to be expressed in mechanical terms using the available input techniques, such as a keyboard or mouse (Norman, 1986). This is known as the gulf of execution (Norman, 1986). This gulf of execution exists as the distinction between expressing a goal or action and how it physically has to be done.

To enable the user to evaluate the effect of their input, they interpret the system actions and states, and compare the apparent system state to their original goals. This is known as the gulf of evaluation (Norman, 1986). These gulfs of execution and evaluation can be overcome to an extent by matching the input and output characteristics of the system more successfully to the user needs, and by adjusting goals and intentions to match the system goals more closely (Norman, 1986).

New technologies such as eye and gaze-tracking, and lip-reading, (Baluja \& Pomerleau, 1994; Bodenhausen \& Manke, 1993a; Bregler et al, 1993a; Bregler \& Konig, 1994; Hanne, 1992) are being developed to provide new interactive human input mechanisms, which more clearly match the natural communication activities of human beings. It is interesting to note that computers do not respond equivalently for interactive output in this area, that is, computers do not 'observe' users through cameras (Bernsen, 1995). 
Other recent developments include touchscreens, speech recognition systems, and recognition systems for two and three-dimensional gestures. Although speech recognition systems can now provide recognition rates of $90-100 \%$ (Fishman \& Padilla, 1997) (in ideal conditions), spoken language interpretation remains ambiguous at optimum recognition levels. Ambiguity can be present in speech even for human-to-human communication. Flexible combinations of speech with other input modes, more suited to human abilities (e.g. gesture, handwriting, gaze-tracking modes), are not yet a desktop convention for human-computer interaction.

While speech recognition systems have now advanced in development to provide affordable, continuous-speech recognition applications, gesture recognition systems (especially those of actual body movements i.e. three-dimensional) are still in exploratory research and development stages (see Wachsmuth \& Frohlich, 1997). In addition to providing a more natural interaction, the use of multiple input modes also enhances the input recognition task, and can serve to resolve ambiguities in the input through the provision of additional information in other modes (Caloini \& Tosolini, 1994; Cassell et al, 1994; Edwards, 1992; Koons et al, 1993; Oviatt, 1997; Oviatt \& Olsen, 1994).

Input in human-computer systems therefore is not so well adapted to the capabilities of human beings (Buxton, 1987). The standard input methods used, such as text input through the keyboard, and pointer and location information from a mouse, do not provide the optimum, natural, communication environment. Contrasted with output media, input mechanisms are poorly matched to human requirements. This is a generally held belief:

'Relatively primitive input devices and interfaces, such as keyboard and mouse still dominate as interface.' (Waibel et al, 1994)

'In general, input has been neglected in relation to output'. (Buxton, 1987) 
In contrast, the communication of output information from a computer to a human being, is much more closely matched to traditional human-to-human forms of communication (Alty, 1991). We can receive information and observe feedback from a computer or machine through mechanisms already well established in human-human communication via text displays, graphical displays, written language, diagrams, real-time video, non-speech sounds, and audio warnings. Speech synthesis systems have been available for some years, for disabled users (usually text-to-speech systems) and there have been some applications which have incorporated speech for both input and output (Lu et al, 1995; Wolf et al, 1995).

However, not all possible modes of output have been exploited in conventional desktop systems. For instance, few man-machine systems include any significant tactile or haptic feedback, as standard, although there have been some research systems developed (Brooks, 1990; Defresne et al, 1995). Nor do current computer systems create output through gesture:

'..when it comes to gesture or bodily movement, it seems clear that current computers do not communicate information to us in these ways.' (Bernsen, 1995).

\subsection{Multimodal Systems}

\subsubsection{Position of this Research}

Many researchers have concentrated on developing novel and efficient ways of combining input media, without considering the interaction framework or analysing how the components combine (Ando et al, 1995; Ando et al, 1994; Bos et al, 1994; Bregler \& Konig, 1994; Bregler et al, 1993b; Duchnowski et al, 1995; Hunke \& Waibel, 1994). Most research in the field of modal combination examines the improved recognition performance gained through the use of multiple input modes, or presents subjective user reviews of the use of multiple modes, without considering why certain combinations of modes and tasks work 
well together (Akamatsu \& Sato, 1994; Vo et al, 1995; Vo \& Waibel, 1993a; Vo \& Waibel 1993b; Vo \& Waibel, 1996; Waibel et al, 1994). These examples are typical of the general trend of research in this area and it has been noted that empirical research and guidelines are lacking (Oviatt et al, 1997).

However, no attempts have been made to properly analyse the effect of redundancy: the result is a lack of guidelines for multimodal and multimedia interface design, evidence about the efficacy of multimodal systems, and guidelines for matching modalities to tasks. Consider these (still) relevant quotes from Alty, Bernsen, and Oviatt respectively:

'Much existing multimedia research has been disappointing partly because of a concentration on exploratory presentation approaches rather than on investigations of how media in combination can improve human computer bandwidth.' (Alty, 1991)

'...a systematic basis for analysing arbitrary input/output modality types and their multimodal combinations as to their capabilities of information representation and exchange is still lacking.' (Bernsen, 1995).

'Currently, little parallel work is available on modality integration during human-computer interaction, although such work will be crutial [sic] to guiding the design of planned multimodal systems.' (Oviatt et al, 1997)

The investigation of the effect of redundancy across modes presented in this thesis, involves an analysis of how additional information from other parallel modes can improve the input recognition task. Redundancy is suggested as the reason for the improvements seen in multimodal input recognition rates when compared with unimodal rates alone (see Akamatsu \& Sato, 1994; Ando et al, 1995; Ando et al, 1994; Bellalem \& Romary, 1997; Bellik, 1997; Bolt, 1984; for some examples of multimodality and redundancy). This thesis therefore attempts to address the lack of empirical data in the area by investigating the role of redundancy between speech and gesture input modes. 


\subsubsection{Research Overview of Multimodal Systems}

Most of the research in this field can be characterised by three main themes.

- the identification of redundancy as a factor where multimodal recognition rates have been observed to be higher than unimodal recognition rates

- the development of multimodal input systems, divided into two categories:

- exploratory research, focusing on system descriptions and architecture

- empirical research, some focus on performance of system after development

- the investigation of how humans integrate modes during human-computer interaction, usually studied by the use of simulated multimodal input systems ('Wizard of Oz' studies)

Research has identified redundancy as the pivotal factor allowing multiple modes to provide improvements in recognition rates over single modes (Ando et al, 1994; Nakagawa et al, 1995; Vo \& Waibel, 1996). However, these studies did not investigate the role of redundancy. Empirical studies of multimodal systems are rare, and more specifically, the question of how to integrate multiple modes, as well as temporal issues, is a neglected issue. Work is now appearing (Bourguet \& Ando,1997; Koons et al, 1993; Robbe et al, 1997) on temporal issues, and the concepts involved in integration (Faconti et al, 1996).

The temporal issues include the prediction of speech timing in multimodal interfaces (Bourguet \& Ando,1997), and a comparison of the effects of timing in constrained speech input as versus spontaneous speech input (Robbe et al, 1997). Issues in integration include the identification of properties of the fusion process for input modes such as temporal co-ordination, incompleteness, and redundancy (Faconti et al, 1996).

There are also a large number of studies involving the use of simulated applications. Simulated systems provide an opportunity for gaining research results by implementing only a front-end interface, using human 'wizards' or 
experts to simulate feedback to users. Wizards perform an integrator role for modal information and a decision maker for feedback to subjects, hence the name of 'Wizard of Oz' studies (Dahlback et al, 1993). Such simulated systems are a common theme in the work examining how humans interact multimodally, since the focus of research is concerned with identifying the characteristics of human beings in the interaction. Therefore, any implementation of a working system is not the main purpose of such research.

In contrast, this thesis examines the role of redundancy in the integration of data from multiple input modes, and this requires the implementation of a system. It is therefore relevant to examine the nature and performance of an implemented multimodal system.

For the reviews of the systems which follow, research in this area has been divided into the following groups and subgroups:-

- Multimodal applications

* Early multimodal input systems

* Empirical research

* Exploratory research

- typed natural language input

- speech and gesture input

- assistive technology (including hand-tracking, face-tracking, gazetracking combinations)

- Multimodal integration

* Integration by the system

* Integration by human beings

- preferred use of modes

- error resolution

- integration patterns 
- Component modes

* Gesture recognition

- two dimensional gesture input systems

- methods for two-dimensional gesture recognition

* Speech recognition and conversational systems

- Constrained and spontaneous speech input

- Multimodal design

* Architecture

* Formal methods

* Software design

- Redundancy

\subsubsection{Multimodal Applications}

Recent multimodal interface research has developed a variety of systems for discovering, noting and interpreting the intentions of users. For instance, a number of input methods have been exploited:

- face and attention tracking through the use of cameras connected to the computer

- eye-tracking

- speech recognition

- lip-reading

- gesture recognition including handwriting recognition

These techniques have been examined singularly, and in combination, to investigate the benefits that they may provide in a multimodal interface. Many have concentrated on particular combinations of input media, some concentrating on a speech and gesture input combination (Waibel et al, 1994), while others have considered force and acoustic/audio output combinations, aimed at blind or visually occupied users (Defresne et al, 1995; Kelley et al, 1995; Ramstein, 1995). However, while much current work has investigated the effects of 
combining modalities, there is little actual objective data and analysis of the benefits.

\subsubsection{Early multimodal input systems}

One of the earliest multimodal research studies was the 'Put-that-there' study by Richard A. Bolt at the MIT Architecture Machine Group Laboratory founded in 1968 (Bolt, 1980; Bolt, 1984; Bolt, 1987).

The 'Put-that-there' system was a speech and gesture prototype system where users were able to create and move a number of objects around a screen. A variety of actions could be performed on the objects e.g. change their colour, shape, position or remove them altogether. The user issued spoken commands via a microphone to a speech recognizer and could issue gestures via a pointing device, similar to a watch attached to the wrist, whilst seated in front of a large screen. The environment was a special room (called the Media Room) designed specifically for the system. The designers wanted users to feel as if they were surrounded by the computer rather than seated facing it (Bolt, 1987).

Speech and gesture input were considered together (in parallel) in order to interpret the user's intentions. Thus some input information could be redundant at times i.e. an action could include pointing to a green circle while saying 'Delete the green circle'. The system has twice received input about the green circle. However, if the speech was not clear on 'green circle' and the pointing was detected somewhere near the green circle but not directly at it, then the two sources of information can be combined to attempt to overcome the ambiguity of the input.

'In other words, the data taken separately from either mode are ambiguous. Combined, though, the evidence gains strength.' (Bolt, 1987)

Bolt goes further with his ideas on multimodal interfaces by suggesting 'selfdisclosing' systems, that would remove the need for manuals and vast quantities of printed material. The critical thing about such a system would be that 'this 
system would interact with its user just as one person would interact with another.' (Bolt, 1987). Bolt's vision encompassed totally natural interaction with the computer. Anything a human being could normally do would be received and interpreted by a computer of the future, providing intelligent feedback eliminating the need for a human being to know how to manipulate any particular computer system (Bolt, 1987).

The 'Put-that-there' study was therefore an exercise in natural, conversational interaction. It was programmed to infer possible conclusions based on the context of speech and gesture input, the current state of the system (e.g. existing objects, position of objects etc.). In cases of continued doubt it would question the user (e.g. "What direction?" would be asked if that part of speech or gesture could not be interpreted) (Bolt, 1987). A user of the system commented that interacting with it was

'..like dealing with a friendly, slightly deaf butler...From the standpoint of your expectations, you are willing to deal with it.' (Bolt, 1987)

No evaluation of the system appears to have been performed (Bolt, 1980; 1984; 1987), although development of this system demonstrated several points relevant to multimodal interfaces.

'Concurrent information in other modes - such as pointing and gesture - can amplify and clarify what is said. Conversely, speech can clarify and give meaning to gestures.' (Bolt, 1984)

This early example of a multimodal input and output computer system identified the key factor: that the parallel use of multiple sources of input provides an opportunity for improving communication success between user and computer, achieved through the resolution of ambiguities between the two sources of information (the natural expression of actions using the two modes of speech and gesture). 
Bolt's vision has not yet been attained. For approximately ten years following the first presentation of 'Put-That-There' in 1980, most research concentrated on the individual components of multimodal interfaces rather than on integration. Thus, speech recognition technology on its own has progressed significantly. More efficient and accurate recognition algorithms have been developed, many utilising Hidden Markov Models, producing commercial speech recognition packages which can operate realistically on desktop computers. Virtual Reality has emerged as a field, focusing on human body movement input. Gesture recognition including handwriting recognition has developed and input devices such as touch sensitive tablets and screens are now readily available. Systems using light pens and touchscreens are used for simple diagnostic systems in hospitals, etc.; with speech recognition systems being used in hands-busy applications etc.

Rudnicky and Hauptmann (1992) carried out research on speech input in multimodal input systems. They noted that the presence of other modalities, in addition to speech input, could provide necessary error correction capabilities. This was necessary since recognition of speech input can be particularly prone to errors. They also made a study of user preference, using the input modes of speech and three-dimensional gesture in a series of experiments. Subjects performed a graphic image manipulation task, using speech alone, gesture alone, or a combination of speech and gesture. They were observed by a wizard who interpreted their actions, and then initiated feedback. From these studies, they found that $58.8 \%$ of subjects preferred the combined use of speech and gesture. In those experiments where users could choose between the unimodal or multimodal use of speech and gesture, $70.6 \%$ of subjects used the multimodal combination of speech and gesture.

This study concluded that the integration of multiple modes of input represented a key goal for the creation of an optimum communication environment, providing more natural communication and complementing speech input as a single mode. Similar conclusions have been drawn in multimodal input studies using combinations of speech recognition and hand-tracking (Hanne \& Bullinger, 
1992), and text input and two-dimensional gestures (Weimer \& Ganapathy, 1992).

Multimodal systems using speech and pen input have been implemented for medical teaching applications, interactive map systems and air traffic control systems (Billinghurst et al, 1996a; Billinghurst et al, 1996b; Chatty \& Lecoanet, 1996; Moran et al, 1998). Other systems incorporating combinations of speech recognition, and two-dimensional gestures have been implemented.

\subsubsection{Empirical Research}

Nakagawa et al (1995) built a robot control simulation system where the user controlled a robot of the system by giving commands to a robot representation on screen. The system was evaluated by noting the performance standard reached by users on attempting specified tasks. Comparisons were made between tasks performed by touch only, speech only and touch and speech combined. Input time, number of input commands, number of misrecognized commands and, where appropriate, the number of 'second best' options chosen were noted. The results are shown in Table 2.

\begin{tabular}{|l|l|l|l|}
\hline & TOUCH only & SPEECH only & $\begin{array}{l}\text { TOUCH \& } \\
\text { SPEECH combined }\end{array}$ \\
\hline Input time (mins) & 19 & 26 & 22 \\
\hline Input commands & 129 & 135 & 112 \\
\hline Misrecognised commands & 4 & 17 & 11 \\
\hline$\%$ misrecognised & $3.10 \%$ & $12.59 \%$ & $9.82 \%$ \\
\hline 2nd best options chosen & not applicable & 9 & 5 \\
\hline
\end{tabular}

Table 2: Average values for Touch, Speech and combined touch and speech (from Nakagawa et al, 1995).

The combination of touch and speech had an average input time greater than that of touch alone, but less than that of speech alone. The number of input commands was less for the bimodal input in comparison to either of the unimodal inputs. Note that the percentage of commands that were not recognised properly was less for the bimodal case in comparison to the unimodal case $(9.82 \%$ for touch and speech combined in comparison to $12.59 \%$ for speech alone). However, the percentage of commands misrecognised was lowest for touch input 
only. This demonstrates the overall reliability of touch input and its low error rates.

These results show that the multimodal approach out-performed the speech-alone methods. The authors further reported that positioning and recognition errors were resolved with the combination of touch and speech modes, and that overall, the multimodal system showed improvements in error rates and speech recognition speed over their unimodal counterparts (Nakagawa et al, 1995).

Similarly, research in the INTERACT project at Carnegie Mellon University (Waibel et al, 1994), has produced several projects which utilise multiple input modes.

In their initial studies on combining speech recognition and lip-reading, statistics were collected on the separate and combined use of modes:

\begin{tabular}{|l|l|l|l|}
\hline Speaker & Acoustic & Visual & Combined \\
\hline msm/clean & 89 & 32 & 93 \\
\hline msm/noisy & 47 & 32 & 76 \\
\hline mcb/clean & 97 & 47 & 97 \\
\hline mcb/noisy & 59 & 47 & 70 \\
\hline
\end{tabular}

Table 3: Word Accuracy (\%) of Speech and Lip system (from Waibel et al, 1994)

These figures show when both the speech recognition and lip-reading accuracies are fairly low (around 50\%), the combined accuracies, when information from both input types is taken into account, are considerably higher (76\%) than either separate percentages $(47 \%$ and $32 \%)$. The addition of noise to the acoustic data severely degrades the accuracy of recognition. However, the combination of 'noisy' or corrupted acoustic input with visual input still gives recognition accuracy of around 70\% ( see also Duchnowski et al, 1994 for a discussion of related speech and lip-reading studies).

Thus, modalities can combine to reduce ambiguities and in certain cases one modality can compensate for another which has performed poorly. This compensation is the critical point about multimodal interfaces. 


\subsubsection{Exploratory Research}

Most research on multimodal systems belongs in the exploratory categories of development, demonstration and initial presentation of approaches, algorithms and architectures. The systems use multiple modes but also usually include some other aspect, such as the use of knowledge bases (intelligent interfaces), temporal issues, or examination of specific modal combinations (e.g. speech and headpointing, speech and gaze-tracking etc.). We review existing systems in this section, beginning with work from 1992, which examined combinations of conventional modes (typed natural language, command language) through to more recent work using more natural input modes, such as speech and gesture. Finally, recent work describing the implementation and early performance of a multimodal system using speech, two-dimensional gesture and handwriting recognition is discussed.

\section{Typed natural language input}

Kuijpers and Wilson (1992) developed a multimodal interface, to demonstrate possible dialogue between users and the system. The architecture of the system is a set of independent processing modules, which govern the operations in the system. The modes used are natural language, and command language, both through keyboard input, and limited mouse-generated two-dimensional gestures. Menu-based commands and icon selection were also possible.

Speech input or output was not included in the system, and this was acknowledged as a limitation. The authors concluded that the research demonstrated the viability of using a multimodal interface as a tool for investigating interactions between users and knowledge bases. The input devices, and resulting type of modes used in this study, were conventional: keyboard and mouse with feedback on a graphical user interface. The study did not give data on unimodal or multimodal recognition rates. Therefore, no data was available to indicate whether the tasks were carried out with a higher efficiency using the multiple modes of input and output. 
An important part of the research, however, is their method of fusing the information from the inputs. The approach is called 'Common Meaning Representation' which is independent of both the application and the modes involved. All input to the system is translated into its equivalent common meaning representation, and all output similarly is represented in this way. This integrated approach is necessary to enable the system to efficiently process information from multiple modes into a single user intention or action. Interestingly, the system also had the capability to provide feedback (output) in a combination of the (system definition) of modes: graphical, iconic, text.

This shows that research into multimodal systems need not involve 'novel' forms of input. Whilst the exploratory development of systems is useful, this type of work illustrates the lack of specific focus in the area. As so many applications have been developed, some inevitably repeat earlier work (see also Bos et al, 1994; Hanne, 1992 for similar types of applications, integrating typed natural language, graphical manipulation and command language or typed natural language and mouse-generated two-dimensional gestures).

\section{Speech and gesture input}

Ando et al (1994), describe a prototype multimodal interface. In their study they compared three input means: pointing gestures alone, command utterances and pointing gestures, and sentence utterances with pointing gestures. Pointing gestures are two-dimensional gestures made through a touch sensitive touch panel. Users were asked to perform organisational tasks in an application 'Interior Design System', in two experiments. The first experiment used software to perform the speech recognition, whilst the second experiment used a 'Wizard of Oz' format (Dahlback et al, 1993). This guaranteed 100\% speech recognition accuracy for the second experiment.

Evaluation results based on the preference responses of users indicated that for the first experiment, the combination of command utterances plus pointing gestures was the favoured input technique. In the second experiment, when 
speech recognition was simulated to be $100 \%$, the preferred input technique was sentence utterances plus pointing gestures. Therefore, the study indicated that natural speech input (the sentence utterances) was preferred when the speech recognition accuracy was perfect, and also indicated that multimodal input was preferred over the use of pointing gestures alone. However, no recognition results were given for the first experiment to allow a comparison or analysis of possible multimodal recognition rates.

Again, along similar lines, the CoSMoS system (Ohashi et al, 1995) is a prototype multimodal interface system which was designed to enable input via speech and three-dimensional gesture. The paper discusses the implementation of the system, and detailed its use in the manipulation of 'blocks' in a building environment. No evaluation or recognition data was presented, although the authors concluded that this system presented a more comfortable communication space for humans since

'A combination of these two modalities can provide a powerful and flexible communication environment.'

Another multimodal input system is that of Koons, Sparrell and Thorisson (1993), where the simultaneous integration of speech, gaze and hand gestures has been implemented. The focus of this research is the simultaneous processing of these inputs, performed freely (no time or order constraints) by users, into a single integrated intention or meaning, which is then compared to information from a knowledge base of the system. At the level of the single inputs, information from each mode is time-stamped and then processed into frame-like structures. Frames from each mode are then processed together, evaluated and resolved to produce a conclusion, to which the system could respond.

They concluded that most user messages could not be interpreted without the information from other modes to resolve the meaning. They also concluded that the opportunities presented by a free combination of input modes '..opens up a highly flexible communication style for the user...' (Koons et al, 1993). This 
research therefore illustrates the ease and flexibility made possible by the use of speech, hand gestures and gaze-tracking for simultaneous input. However, there was no evaluation of the system performance, or any comparisons against single modes for efficiency ratings.

Further work on the use of multimodal input in an intelligent environment can be found in Billinghurst et al (1996b). This system involved the use of speech and three-dimensional gesture input in a simulated surgical training environment. With the aid of an expert system which provides context knowledge for interpreting the multimodal input, and a knowledge of surgical procedures, an intelligent medical training tool was developed (Billinghurst et al, 1996a). Again, this work shows the advantage of a common method of representing the integrated input in order to resolve the meaning from the individual modes, in this case, the representation used was conceptual dependency frameworks (Schank, 1972). There was no evaluation of the system to indicate if there were improvements in the efficiency of multimodal input over unimodal. The authors, however, claimed that the multimodal approach provided more reliability in the interface through improvements in input recognition accuracy.

Waibel et al (1997) have developed a comprehensive multimodal system using speech, two-dimensional gesture, handwriting and face-tracking input modes. Again, the fusion process is similar to the other research examined, where the processed information from the input modes is represented in slots within partially-filled frames. The frames are the merged to give the combined multimodal interpretation. They argued that this approach provides uniform handling of all high level information giving modularity, and the possibility of extending the system to incorporate other input modes later.

Waibel et al (1997) examined the potential for error correction and flexibility which multimodal redundancy offers. They suggest that this might be exploited through user involvement with the system. The user, for example, could choose from a list of recognition candidates, or select a preferred way of correcting errors 
(by using a particular mode to provide correction information). No user evaluation of the system was carried out.

Related to the work of Waibel, is that of Vo and Wood (1996) who describe the implementation of a multimodal framework for the integration of speech, twodimensional gesture and handwriting recognition. The integrator module again uses semantic frames to merge the input from the individual modes into a single multimodal representation, in an interactive appointment scheduling application. Pilot experiments were conducted using the Wizard of $\mathrm{Oz}$ format, to gain examples from subjects of interactive use of the system. This provided a set of speech-only, gesture/handwriting-only and multimodal examples which were then treated in two separate ways.

Firstly, the examples were transcribed to provide a set of perfect inputs, representing what each interaction was, including the exact phrases spoken, gestures and handwriting input made. Additionally, the examples were processed by the individual modality processors (speech and gesture/handwriting) to provide a set of recognised inputs. Secondly, the perfect input was processed by the multimodal interpreter, followed by processing of the recognised input, which gave a comparison of the multimodal interpreter performance in the presence of correct input (the perfect input), and in the presence of recognition errors from the separate modes (the recognised input).

The multimodal interpreter made the correct conclusion in $80 \%$ of cases for perfect input, and in the range 35-62\% for imperfect input. Therefore, it could be concluded that the multimodal interpreter had fair performance, but would require improvement since perfect input is very unlikely in actual working systems. They also noted that lower interpretation errors occurred in the combined, multimodal examples. The recognised speech-only examples gave an error rate of $35 \%$ compared to only $12 \%$ from multimodal examples in one experiment, while another experiment gave interpretation errors from pen-only examples at $61 \%$, in contrast to the multimodal error rates of $49 \%$. 
Their work again stresses the importance of both complementary and redundant information from multiple input modes. They also hypothesised that the presence of redundant information across the modes might have contributed to the lower interpretation errors seen in the multimodal examples, compared to either the speech or gesture/handwriting examples. However, they were unable to analyse this hypothesis further within the presented experiments. It is exactly this concept, the compensation and reduction of possible error, which we examine later in this thesis (Chapter 8).

\section{Assistive Technology}

Another area in which multimodal input has implications, is that of assistive technology (i.e. specifically for users with impairments or disabilities which may prevent them from using standard input devices such as the mouse and keyboard). There have been studies conducted for those users with speech skills ranging from relatively unimpaired up to severe speech impairments, which propose that the integration of speech input and head-pointing input could compensate for those unable to use the mouse for pointing and spatial input (Smith et al, 1996). This study has not unfortunately advanced to the stage where the two inputs have been integrated.

There has also been a study examining the use of two types of gestures for multimodal input, for users with some motion impairments (Keates \& Robinson, 1998). The study looked at the use of input from both the head and the hand in a number of ways that could possibly improve the interaction process firstly by providing a choice of input mode to the user, secondly by requiring input from both gesture modes simultaneously to increase the recognition accuracy (i.e. redundancy) and finally, by implementing a complementary set of inputs from both gesture modes. The results of the study indicated that, for impaired users, multimodal input presented particular difficulties, such as cognitive overload, as well as the necessity of producing sometimes unfamiliar physical movements. This is not surprising given the different requirements and conditions required by impaired users. The authors concluded that gesture as part of multimodal input 
was feasible for impaired users, although consideration of cognitive and learning demands was obviously necessary.

The studies outlined above give an overview of the scope of multimodal systems, and their possible application for those users with impairments that limit their full use of conventional systems.

\subsubsection{Multimodal Integration}

\subsubsection{Integration by the System}

A formal framework has been proposed by Faconti et al, 1996 which describes an investigation of the fusion of input modes. They describe the problem of how the information from several input modes needs to be integrated into a single (multimodal) representation of all the information, which can be processed by the application. Their approach is based around formal process modelling where they maintain that, if the process of fusion is formally stated, this creates

'...a single clearly defined fusion process that behaves consistently across applications...' (Faconti et al, 1996).

In particular, they argue that a formal methods approach allows the proof of processes in a specification and examination of the truth of the properties of these processes. Specifically, they prove the validity of the properties of the fusion process, namely, incompleteness, redundancy and temporal co-ordination.

The property of incompleteness is described as the ability, of the fusion process, to deal with possibly incomplete data, in order to provide a conclusion of some kind. Redundancy is described as the property of repeated information which can allow for the disambiguation of possibly inconsistent input. Finally, the temporal co-ordination property specifies three types of fusion trigger (that is, when the fusion should occur). Firstly, a particular type of input could act as a trigger for fusion to occur (e.g. always fuse data after gesture input). Secondly, a condition could be set where fusion occurs only when a complete defined set of input has 
been made. Thirdly, a constraint could be set whereby fusion only occurs on input that has been made within a specific interval of time.

Although the formal methodology is not easy to comprehend, these definitions are very relevant. In particular, they conclude that they have proven that both the redundancy and incompleteness properties are unavoidable in multimodal systems, and must be dealt with in the architecture of the multimodal system.

An area from which new ideas may be gained is that of sensor fusion. Data/sensor fusion examines the integration of various forms of data, usually in engineering and control applications (e.g. robotics). Although the technical vocabulary differs, the basic concepts between data fusion and the integration of multiple modes remains the same. The benefits and complications of data fusion have been noted, and are similar to those complications that the use of multiple modes give for recognition tasks. A reduction in error rates for recognition tasks has been seen when data from different devices has been fused in surveillance applications, using acoustic and radar data (Zemany et al, 1993).

Fusion is also necessary in target recognition applications where data from only one sensor is insufficient to identify a target, again a factor in common with multiple input modes, where a single mode may not contain sufficient information for task completion. The temporal issue is also present in the sensor fusion area, where the question of when to fuse the data arises (as there is the possibility of information from individual sensors being incomplete due to differences in sensor operation). The integration methods used in sensor fusion span the areas of neural networks, bayesian networks, and probabilistic methods (Zemany et al, 1993). However, perhaps the difference in nature of the entities to be fused appears to prevent further comparison of integration methods, as little acknowledgement of similarities between the fields appear to have been made.

There has also been work done by Nigay and Coutaz (1993), who produced a classification system for multimodal interactive systems. They define three levels of fusion: lexical, syntactic and semantic, where lexical fusion is concerned only 
with temporal fusion and data synchronisation, syntactic fusion involves the combination of data to obtain a complete command, and semantic fusion combines the results of commands to produce new interpretations. The work also notes that fusion can be performed with or without knowledge of the type of data being combined.

For instance, fusion using only temporal information does not require knowledge about the components or the overall aim of the fusion process. By contrast, fusion using modes such as speech and gesture, where the possibility of anaphoric references arise (e.g. put-that-there), does require knowledge of the overall structure and result that needs to be produced. Therefore, in the fusion process, two main criteria are specified, the temporal (time-stamping) issue, and the structure of the objects being combined. The model proposed therefore covers the main aspects relevant to multimodal input systems, the temporal aspects of fusion and the necessity for knowledge of the data being combined.

It is interesting to note a common factor among the papers mentioned in this review. The research discussed has spanned almost two decades, from the presentation of 'Put-that-there' in 1980, through several studies in 1992, to more recent work from 1994, 1995 to the present. Most of these systems which have described their process of fusing the input data together, have all adopted some type of technique similar to natural language parsing methods, such as frames, conceptual dependencies, common representation formats etc. Perhaps this commonality can be attributed to the fact that such methods are the best way of representing information from several modes in one representation. The flexibility provided by this method is the overwhelming factor in its favour.

\subsubsection{Integration by Human Beings}

Research has been performed on the temporal aspects of modal integration by human beings. Bourguet and Ando (1997) conducted experiments to analyse the relationship between speech and pointing gestures during multimodal interaction. The aim of the study was to produce a quantitative model that could be used in 
the design of future robust multimodal systems. The study found that there was a synchronisation between the end of pointing gestures and the part of speech relevant to the pointing (deixis). In particular, the end of the pointing gesture was usually just slightly before the relevant speech part occurred.

The research concluded that speech timing could be predicted according to gesture timing which can be useful for future applications using speech and pointing gestures as input. The ability to predict the timings is particularly beneficial for reducing the possibility of error during the integration process, when the issue of when to integrate can be problematic for a sensible combination of modal information.

Substantial work has been performed by Oviatt et al (Oviatt, 1996; Oviatt et al, 1997; Oviatt \& Olsen, 1994; Oviatt \& Van Gent, 1996) on the ways in which humans actually integrate multiple input modes during human-computer interaction. There are several areas of focus in their work: firstly, they have examined the human preferences in use of multimodal input modes in specific application domains (Oviatt, 1996); studies have also focused on error resolution during multimodal human-computer interaction (Oviatt \& VanGent, 1996); and thirdly, patterns of human integration and synchronisation have been examined (Oviatt et al, 1997; Oviatt \& Olsen, 1994). Each of these areas will be discussed in the following sections.

\section{Preferred use of modes}

In a study examining the use of multimodal input in applications using 'interactive maps' (for geographical information systems), a simulated experiment was performed to analyse the human use of input modes (Oviatt, 1996). Possible modes were speech input and pen-input which included handwriting, two-dimensional gestures, symbols and other marks on the application display (maps with varying levels of detail). The multimodal input therefore consisted of a mixture of speech and pen input, with subjects provided with a free choice of which to use, either unimodal or multimodal. 
Results from the experiment indicated that, in comparison to speech-only input to a map, the combined use of speech and pen input decreased the task completion times, reduced error rates, and simplified the linguistic expressions necessary in the input (as pen input reduced the need for complex speech utterances in order to complete tasks). Additionally, $94.5 \%$ of subjects (17/18) preferred interacting multimodally with the map, while only one subject preferred unimodal written input, although none expressed a preference for unimodal speech input only. This study indicates the benefits of multiple input for the task recognition efficiency and also concludes that due to the relative complexity of multimodal interfaces, further empirical assessment would be useful for guiding future design.

\section{Error resolution}

One observed benefit of interacting multimodally is the reduction of error rates in task recognition. Further work by Oviatt and VanGent (1996) has analysed the tactics adopted by humans, during human-computer interaction, to correct recognition errors made on speech input. They also note that error avoidance can also occur during human-computer interaction, as humans appear to intuitively choose modes best suited for the tasks (e.g. the choice of writing a foreign surname as versus speaking it). Again, the study involved a mock application, a service transaction system, where errors in recognition were simulated in order to force subjects to adopt different strategies to obtain input recognition success, using speech and pen inputs, as in previous studies.

The results of the experiment indicated that, when apparent recognition errors were encountered, users would repeat input using the same mode, and if further recognition errors occurred, they would change to another mode of input. In only $0.5 \%$ of cases were both modes used simultaneously for specific error resolution activities. This research therefore indicates patterns of multimodal interaction during error resolution. However, the results cannot be used to conclude anything further about other activities outside error resolution. 


\section{Integration patterns}

Studies have also been conducted to analyse how human beings intuitively integrate their input during human-computer interaction. The combination of spoken and written input was examined in two simulated applications (Oviatt et al, 1997; Oviatt \& Olsen, 1994) with the common aim of providing guidelines for the design of robust multimodal input systems, to support natural human behaviour in human-computer interaction.

The first of these studies (Oviatt \& Olsen, 1994) investigated how humans used speech and pen input in a simulated service transaction system (see Oviatt $\&$ VanGent, 1996). Results indicated that several factors affected the choice of modes: task content, presentation format and contrastive functionality. In task content, digits were more likely than text to be written instead of spoken. For presentation format, form-based presentations attracted more written input than unconstrained presentations. Contrastive functionality refers to those cases where spoken input was used first, followed by pen input, or vice versa, the change in mode is attributed to specific changes in intent e.g. making a correction to entered input, changing from data input to command input, and the input of digits instead of descriptive references.

There was no objective analysis in the study of the combined use of speech and pen input. The subjects indicated an overall preference for multimodal over unimodal input, especially in the cases where the simulated task involved the use of digits. There was little evidence of the simultaneous use of spoken and pen input, although where this did occur, the task was focused on digits. However, there was no reported analysis of the observed use of both modes, the focus being on non-simultaneous use, for a single task.

The second of the studies (Oviatt et al, 1997) conducted an analysis of the multimodal interaction of humans. This study uses a combination of systems from earlier reported work, the simulated interactive map application (Oviatt, 1996) and the service transaction system (Oviatt \& Olsen, 1994). In this study, 
the combined use of spoken and pen input was examined, to determine when users were most likely to use multimodal input over unimodal input.

User patterns of interaction indicated that multimodal input was preferred over unimodal input, with all subjects using both spoken and written input at different points for the same task, and also reporting that they did prefer using multiple modes over single modes. The type of task involved also affected the likelihood of it being expressed multimodally. For instance, those tasks which had a spatial location content were most likely to be performed multimodally, involving graphic input to add, move, select or modify objects of interest. Results also showed that there was a tendency for duplicate information to be provided in instances where object identification or selection was involved. However, for the spoken and written modes, they concluded that overall, different linguistic information was supplied from both modes rather than redundant information across modes (see Section 3.5 for further discussion of this result).

The research of Oviatt $e t$ al, (1995 to 1997) provides the following conclusions.

- $\quad$ preference of subjects for using multimodal over unimodal input;

- detailed analysis of the synchronisation of spoken and written input;

- $\quad$ pre-knowledge of possible task commands is a valuable factor for the design of multimodal systems, particularly for the system integration of modal information, as predictions can be made of where each incoming input belongs, its context and its relationship to other input.

\subsubsection{Component Modes}

In a multimodal input system, there can be several component modes, and the inputs from these are integrated into an overall multimodal input stream. As we have seen in earlier sections, there are a variety of different possible input modes. For instance, speech recognition systems have been widely available for many years, various gesture technologies are being developed (two-dimensional gestures from basic drawing to handwriting recognition, and three-dimensional gestures from hand movement recognition through to whole-body tracking (Bordegoni \& Faconti, 1996)). There have also been developments in gazetracking, face-tracking, and lip-movement tracking, which can be combined with 
speech recognition to form 'speech-reading' systems (for examples of various input systems see Baluja \& Pomerleau, 1994; Bodenhausen \& Manke, 1993a; Bodenhausen \& Manke, 1993b; Bregler et al, 1993a; Duchnowski et al, 1994; Hunke \& Waibel, 1994; Manke \& Bodenhausen, 1994; Manke et al, 1995a; Manke et al, 1995b; Schiele \& Waibel, 1995; Weimer \& Ganapathy, 1992; Zhao et al, 1995).

In the following sections, the discussion will focus on the input areas of gesture recognition and speech recognition, which are of relevance to this thesis. In gesture recognition, the core of discussion is on two-dimensional gestures, as this is the type used in the research presented here. See Wachsmuth \& Frohlich (1997), Yang et al, 1998, Baluja \& Pomerleau, 1994, Hunke \& Waibel, 1994, Schiele \& Waibel, 1995 for an overview of three-dimensional gesture recognition incorporating the recognition of a wide variety of human movements. Most are body-part specific: for example, hand movement recognition especially for sign language applications; head-pointing; eye-tracking for monitoring of the gaze; lipmovement; pointing gestures.

A method of two-dimensional gesture recognition will be discussed, studies examining application areas of gesture recognition presented, and a brief discussion of speech recognition systems will be covered. Also, as a commercial speech recognition system was used for this research, the actual methods used in speech recognition are not covered in depth, although topics relevant to this research are briefly discussed (e.g. constrained versus spontaneous speech input).

\subsubsection{Gesture Recognition}

Two-dimensional gesture input systems

The earliest, and still prevalent, form of gesturing is that of two-dimensional drawing on a visual display, such as a pressure-sensitive touchscreen (or on graphic tablets). The drawing of marks on graphical interfaces arose out of a trend away from command language based systems. The development of the 
graphical user interface and the wish to be able to interact more naturally with systems, led to the advent of 'direct manipulation interfaces' and an associated ease-of-use (Shneiderman, 1982).

Early studies using two-dimensional gestures focused on their possible use for text editing tasks, and in particular, on the comparison of the use of gestures with keyboard and mouse combinations (Mack \& Lang, 1989; Mahach, 1989; Wolf, 1992; Wolf \& Morrel-Samuels, 1987). For instance, in a study examining the use of hand-drawn gestures for text editing using pen and paper, it was found that subjects were consistent in their use of editing gestures (Wolf \& Morrel-Samuels, 1987). Subjects also indicated that they found such gestures easy to use and remember. The study therefore concluded that machine recognition of gestures was a feasible option because of the regular behavioural patterns of users employing gestures, although noting that not all aspects of a pen and paper study would necessarily transfer to interaction in a human-computer system.

Further work from this study documented a comparison of gestural, keyboard and mouse interfaces (Wolf, 1992). In a spreadsheet application, two-dimensional gestures were used for command input, and handwriting input, and compared to a conventional system using keyboard and mouse. Two combinations of input and output hardware were used for the gestural interface. The first combination allowed the gesture input to be drawn on a transparent tablet mounted to the output display, so that it appeared that the user was gesturing directly onto the display. In the second combination, an opaque graphic tablet for input and a separate output display was used, so the user's input was performed on one device and feedback and output were presented on a different device (i.e. monitor).

Results of the experiments indicated that the time taken to complete application tasks was significantly shorter using the gestural interfaces, than through the conventional system using both keyboard and mouse. There was no significant difference in completion times between the two types of gestural interfaces, although subjects indicated their preference for the transparent tablet, where 
gesturing was performed directly onto the screen. Overall, the study concluded that these gestural interfaces had more benefits for ease of use than the keyboard and mouse interfaces, particularly because gesture required fewer operational steps to complete tasks, and the monitoring of input actions and feedback required less attention by users in the gestural interface.

These studies have demonstrated the feasibility of using two-dimensional gestures in graphical user interfaces, the performance benefits that this gesture mode supplied, and advantages over the conventional keyboard and mouse combinations (see Briggs et al, 1992, for a similar comparison of handwriting input versus keyboard input). The type of gestural interfaces described above were the forerunners of current pen computing. Pen computing exploits the merits of using a pen in computing environments, normally employing a digital stylus on a tablet display. It is commonly used in devices such as Personal Digital Assistants, Digital Notepads, hand-held PCs, and palm tops etc. (see Chatty \& Lecoanet, 1996; Kato et al, 1995; Geissler, 1995; MacKenzie et al, 1994; Manke et al, 1995a; Manke et al, 1995b; Masui, 1998; Zhao et al, 1995, for further pen computing related applications and reports).

\section{Methods for two-dimensional gesture recognition}

There have been studies examining various techniques for two-dimensional gesture recognition (Rubine, 1991). Most research work in this area, however, has usually involved examination of the feasibility of a gesture-based interface for the specific application, without a focus on a gesture recognition system alone (see previous section). Two-dimensional gestures usually take the form of drawing on a pressure sensitive touchscreen with a stylus, and the trace of the pattern drawn is fed back on-screen.

The trace of the gesture is converted into a set of co-ordinates received from the input device, and these are then processed for interpretation. The processing and interpretation forms the basis of the gesture recogniser. Usually gestures are regarded as single-stroke entities, where the start of a gesture is the first contact of 
the stylus to the tablet or touchscreen, and the end of gesture is marked by the cessation of contact with the screen (see Kramer, 1997 and Section 4.3 for further details of two-dimensional gesture characteristics).

Rubine (1991) developed a trainable gesture recogniser for single stroke twodimensional gestures. He acknowledged that there were disadvantages in omitting multi-stroke gestures, for instance, conventional compound strokes, such as crosses $(x)$ and arrows $(\rightarrow)$, were not included. The trainable gesture recogniser can be used to add gesture content to direct manipulation applications. The application must be developed using an associated tool-kit developed by Rubine, so that gesture options can be chosen and incorporated, where desired, in the system. The system must then be provided with examples of the chosen gestures, in order to produce classes of gestures, representing interpreted categories into which recognised gestures can be classified.

For the actual gesture recognition processes, statistical techniques are used. The co-ordinates of gestures are analysed and a set of features calculated, which are combined to produce a feature vector. The feature vector is then placed into one of the earlier defined classes by a statistical classification algorithm. The performance of the system is reported to be in the $96-98 \%$ range, with best performance seen when the number of gesture classes is limited, and the number of training examples per class maximised. The research described an efficient and relatively simple method of gesture recognition, with the added advantage of an available tool-kit for development of new applications incorporating gesture recognition technology.

This system was a possible candidate for use as a gesture recognition system in the eventual multimodal system that was developed for this thesis (see Chapter 6). However, the constraints imposed by the necessity of using the provided toolkit (which was not compatible with our speech recognition development environment), the single stroke constraint, and the necessity of initiating a gesture through a single mouse click ruled out its use. (For further work on the development of two-dimensional gesture recognition systems, see Kramer, 1997.) 
The two-dimensional gesture recognition system developed for the research presented in this thesis is discussed in Chapter 4.

\subsubsection{Speech Recognition and Conversational Systems}

Current technology that supports speech recognition has now advanced to the stage where continuous speech recognition is possible. Users of speech systems can speak at a relatively normal pace, and the systems have reported recognition rates at the higher end of the $90-100 \%$ range. The provision of continuous recognition is relatively recent (see Fishman \& Padilla, 1997), but discrete speech recognition has been widely available for a variety of platforms (PC, and Macintosh) for a number of years. However, speech has yet to become a conventional input mode in our standard desktop systems, although other applications of speech recognition technology have been more widely accepted (dictation for report creation (Lai \& Vergo, 1997; Sharman, 1994), and transactions through the telephone (Wolf et al, 1997; Wolf \& Zadrozny, 1998), reducing the need for employing human operators for transactions such as banking, bill paying etc. ).

The lack of acceptance of speech at the interface has probably resulted from the margin of error that still exists in speech recognition systems, the necessity for repetition to correct errors, and the cognitive load that the use of speech can cause. Much research has examined the possible ways in which speech input can be optimised.

\section{Constrained and Spontaneous speech input}

Natural, fully conversational speech interaction is not yet a realised goal of human-computer interaction. As technology advances with the advent of readily available continuous speech recognition software, the goal becomes closer. However, there are several non-technological issues which can contribute to the development of such interaction, such as an appreciation of how humans interact conversationally and strategies adopted by humans when system recognition 
errors occur. Also when, inevitably, constraints are adopted in systems, whether for processing or management considerations, knowledge of how humans interact within speech constraints also contributes to the development of limited conversational systems.

A study of constrained versus spontaneous speech input in a multimodal interface has demonstrated that when humans are limited to a subset of a given vocabulary (within a natural language subset), they responded positively to the constraints (Robbe et al, 1997). The comparative study noted that hesitations and incorrect utterances were reduced in the constrained speech version of the study. However, these comparative results were limited by the fact that users may have benefited from a training introduction in the constrained study, where a written description of the possible language and vocabulary was supplied. No such training or guidance was given in the spontaneous study. Nevertheless, the overall conclusion is positive from such studies.

Given a 'reasonable' interaction standard, users are compliant and willing to interact within some constraints, which may result in more efficient interaction and improved system recognition through the formulation of more precise, less ambiguous oral utterances (Robbe et al, 1997). It has been reported that in conversational studies of speech input, the longer the utterance, the higher the spoken 'disfluency' i.e. longer utterances tend to have '..increased planning demands' (Oviatt, 1995), leading to an increased chance of spoken disfluencies (Oviatt, 1995; Wolf et al, 1997). Also, a study of conversational interaction in comparison to keypad and keyboard input, has shown that it is not utterance recognition accuracy that can affect user satisfaction, but more the overall success in achieving the system goals (Wolf et al, 1997).

\subsubsection{Multimodal Design}

For user interface design, there are some (almost common sense) accepted rules for the development, layout and function of systems. The 'rules' have evolved from the experience of developing interfaces over a number of years, starting as 
heuristics (rules of thumb) and proven in use and research (Eberts, 1994; Shneiderman, 1998). These cover aspects such as consistency, clarity of display and feedback, and simple and effective handling of errors. These rules can highlight the practice of good design and are practical guidelines in the development of interactive systems.

There are also theories and frameworks for interactive systems, which offer a structure and context for analysis of the systems. For instance, the GOMS model (goals, operators, methods and selection rules) regarded users as the formulators of goals and sub-goals, achieved through the application of procedures and methods (Card, Moran and Newell quoted in Shneiderman, 1998, pp55). Other models have evolved from this work which could be used to predict learning times, performance times and possible errors in interactive systems (Shneiderman, 1998). There have also been action models summarising the stages of action that users go through when interacting with systems, which can help predict the steps taken by users, and patterns of behaviour and error which can arise in the interaction. Use of these models can obviously lead to improvements in design when the cause of user errors can be identified and corrected. Clearly, theories and frameworks only serve a helpful purpose if they can eventually lead to solutions of practical problems.

In the area of multimodal systems, there has been research into models, and characteristics of these systems. While frameworks have been suggested for consistent terminology in the area of multimedia interface design (Frohlich, 1991) the terminology has not yet reached an agreed constant state. Similarly, guidelines for the design and integration of modes in multimodal interfaces are numerous, but not necessarily consistent.

A number of frameworks and theories (Coutaz et al, 1993; Faconti et al, 1996; Hill et al, 1992) have been put forward to assist designers in the development of multimodal systems. They are incomplete and sometimes contradictory, but can be of use in certain defined circumstances (e.g. where they can be readily related to a particular system or situation). Generally speaking, design is a continuous 
process which cannot easily be constrained into a set of guidelines (Shneiderman, 1998).

\subsubsection{Architecture}

Hill et al (1992) discussed the architectural problems inherent in current multimodal interfaces and systems. They identified five qualities that were necessary for multimodal interfaces to become more significant.

- $\quad$ modality blending

- ambiguity resolution and encouragement of ambiguous, probabilistic input

- co-operation protocols in the system architecture to process such ambiguous input

- a modular architecture

- interaction and interpretation histories for evaluation and research purposes.

The authors refer to the necessity of modality blending since

'Multimodality per se is not enough' (Hill et al, 1992)

They describe modality blending as the ability to switch (in a seamless, fluent manner) between modes, at any point during input, as appropriate for the current task or action. Ambiguous and probabilistic input such as whole sentence speech input and stylus drawn gesture input should be encouraged in their view, and is desirable since this type of input is appropriate to certain tasks:

'Designers of previous generations of interface technology worked hard to drive ambiguous and probabilistic inputs out of interfaces because they were unmanageable. The next generation should let appropriate resolvable ambiguity and tolerable probabilistic input back into interfaces.' (Hill et al, 1992)

These characteristics are relevant in the design of multimodal systems. Evidence of ambiguity resolution, co-operation protocols, and modular architectures can be seen in the work of Koons et al, 1993, Oviatt \& VanGent, 1996, Vo \& Wood, 1996, Waibel et al, 1997, and Kuijpers \& Wilson, 1992. Waibel et al (1997) also demonstrate the use of a form of interpretation histories, which can be consulted 
in the course of resolving input information. Their system can consult context knowledge contained in previous interpretation frames to aid the multimodal interpretation process. The concept of modality blending has not received significant attention in implemented systems, although an aspect of the human use and blending of modes has been considered in the work of Oviatt, 1996, Oviatt et al, 1997, and Oviatt \& VanGent, 1996.

\subsubsection{Formal Methods}

There have been studies investigating the application of formal methods for specifying properties of multimodal interactive systems (Coutaz et al, 1993; Faconti et al, 1996). These studies detail how the formal specification of multimodal systems can provide solutions for designers for refinement of systems, and for the association of tasks to elements within systems. The study in Coutaz et al (1993) describes the comparison of two formal notations for specifying properties of systems. However, the application of such notations, through the use of developed specification languages appears to be laborious. Also, the study did not provide conclusions for multimodal systems. Another study also details a formal framework specifically analysing the fusion of input modes in multimodal systems (Faconti et al, 1996). This framework does supply relevant definitions for the area, covering redundancy between modes and characteristics of the fusion process. These have been discussed in Section 2.5.4.1.

\subsubsection{Software Design}

Another study of the design implications of multimodal systems is that of Nigay and Coutaz (1993). In their research they had the aim of specifying multimodal systems from a software engineering viewpoint, in order to identify software constraints and implications for designers. They identify two main properties of multimodal systems as concurrency of processing and fusion of both input and output modes. 
Their definitions are highly relevant to the area and do provide a positive summary of the characteristics and properties of multimodal systems, particularly input systems. They define how fusion in a system (of both input and output modes) can occur with and without knowledge of the meaning of data. For instance, fusion of a soundtrack and associated video footage, for output, can be based on purely temporal information, to provide an adequate combination of two media. In contrast, the fusion of speech and gesture input, in the 'Put-that-there' approach (using anaphoric reference) does require knowledge about the meaning of the data to provide a combined interpretation. A detailed discussion of their fusion theories has been discussed in Section 2.5.4.1 (see also Vetere et al, 1997 for a review of this area).

\subsubsection{Redundancy}

To recall from Section 2.2.4, we refer to redundancy here as the concept of repeated or duplicate information between (across) multiple input modes. There have been other uses of the term, but in this thesis we constrain ourselves to this definition.

There is relatively little work which addresses the concept of redundancy directly. As discussed in Section 2.5.3, there is some research which has identified redundancy of information as the pivotal factor in the increased recognition rates seen in multimodal input over unimodal input. However, studies discussing redundancy in depth have usually been confined to non-computing areas (Basil, 1994; Lang, 1995) although there is some work analysing redundancy in the context of interfaces for users with disabilities (Edwards, 1992). These studies also usually address redundancy from the viewpoint of output channels, rather than input channels.

Redundancy has also been examined in the context of human communication, and in particular written language. Shannon (1951) described a technique for assessing average redundancy levels within written texts. This enabled a measurement to be taken of how much prior information was used by readers 
when interpreting written language. Cherry (1966) refers to redundancy in language as:

'Syntactic redundancy implies additions to a text; something more is said or written than is strictly necessary to convey the message.' (Cherry, 1966)

He also comments that redundancy is perhaps a misnomer:

'..- a rather unfortunate term in view of the important role it plays.' (Cherry, 1966)

The importance of redundancy for error correction can be seen in error-correcting codes, used in the transmission of information. Extra information bits are added to the code to allow the detection, and possibly correction, of errors upon decoding, especially in noisy transmission conditions (Richardson, 1968; Iyanaga \& Kawada, 1977). This use of redundancy has been an important factor in the fields of information and coding theory, leading to the development of robust codes (e.g. Hamming codes, Reed-Solomon codes) which can be used where the efficient and secure transmission of information is required in possibly noisy circumstances (e.g. data transmission across a radio communication link from space).

When considering redundancy across output modes, there have been studies conducted in the area of information processing of television messages (Basil, 1994). Analysis of how much humans can process and learn from viewing television has been studied, and through this, the redundancy of the television output channels (audio and video) has been examined. Although this is in a different area to our area of interest (i.e. input) the definitions and discussion of redundancy are relevant.

Basil (1994) refers to definitions of the overlap of multiple modes used in interpersonal communication (see also Section 3.4.4 for discussion of overlap of multiple input modes). These overlaps are categorised as redundancy, substitution, complementing, contradicting and emphasising. Redundancy is 
defined as one channel repeating what is said in another channel, substitution where one channel replaces what is missing from another channel, complementing is where one channel adds information to the other channel, contradicting is where one channel is the opposite of the content contained in the other channel, and finally, emphasis was defined as the addition of information in one channel to stress what is in the other channel (Basil, 1994, quoting Ekman \& Friesen, 1969).

These definitions are equally applicable to the possible overlaps that may occur between multiple input modes. The investigation of redundancy in these studies generally concludes that redundancy can increase the information transfer to humans, and that there are particular combinations of channel types and presentation types which give optimum transfer rates (Lang, 1995). Therefore, these studies could be viewed as the reciprocal of the goal we wish to achieve here, the investigation of redundancy across input modes, rather than output modes.

Vetere (1997) examines redundancy from the perspective of human-computer interaction. He notes that the nature of redundancy is not well formulated, and reports that there is 'insufficient knowledge' to help designers manipulate redundancy to improve interactions. The report proposes that a framework for modelling redundancy in human-computer interaction will allow for an understanding of the concept, and therefore enable specific design approaches to incorporate redundancy at the interface. This also agrees with the observations of Alty (1991), Edwards (1992), and Marmollin (1991), who acknowledge the importance of redundancy in multimedia and multimodal systems for improving the quality of interaction (both input and output). 


\section{Section 2}

\section{Initial Work}




\section{Chapter 3}

\section{Focusing on Input}

\subsection{Introduction}

This chapter discusses the focus of this thesis: an examination of the role of redundancy between input modes. An overview of input devices is presented, and the possible overlaps that may occur when using multiple input modes are discussed. An outline of the necessary input components for an experimentation system is also given.

\subsection{Input devices and methods}

\subsubsection{Mouse and Keyboard}

Conventional desktop systems support two basic input devices: a keyboard and a mouse pointer. The keyboard is used chiefly for textual data input, while the mouse provides a method of direct manipulation through the feedback of relative position information. Clearly, these types of input devices do not make use of the physical and expressive potential that human beings possess. Buxton (Baecker \& 
Buxton, 1987; Buxton, 1987) illustrated this point by an amusing example. If a physical anthropologist of the future were to unearth a conventional computer system from today's society, assuming that information about us had been lost,

'...we would pictured as having a well-developed eye, a long right arm, a small left arm, uniform length fingers and a "low-fi" ear. But the dominating characteristics would be the prevalence of our visual system over our poorly developed manual dexterity.' (Buxton, 1987)

Buxton pointed out that input had been neglected in comparison to output, and argued if input were more tailored to the needs of human beings, computer systems would be easier to learn, easier to use and provide the possibility of reducing errors (Baecker \& Buxton, 1987). A fundamental example of the limitations of conventional systems is the fact that we use only one hand to provide input (excluding typing). He gives several examples where the use of the 'non-mouse' hand could improve the interaction, by speeding up the process, and prevent the necessity of eye movement between keyboard and display, to check the feedback on data entered.

Recent research simulating two-handed interaction has shown how the natural use of two hands is not exploited by conventional systems (Hummels et al, 1997). For example, the act of scrolling through text could be made easier, by the provision of a mechanism for anticipating selection during scrolling. Entering data into a spreadsheet could benefit from differences in keyboard layout, which would allow selection of cells with one hand (using the mouse) whilst the other hand is engaged in entering the data. Most current keyboard layouts (for righthanded users) place the keypad entry keys near the mouse (Shneiderman, 1998). Three-dimensional gesture recognition may make natural two-handed input a reality, through the ability of providing sign language input to computer systems and the development of natural gesture recognition (Wachsmuth \& Frohlich, 1997). 
Although the argument against keyboards as ideal input devices has been made many times, they still dominate because they are efficient and provide a reliable means of input (Shneiderman, 1998). Developments have led to a variety of different keyboard layouts, optimised for speed of input, and minimising of errors. A keyboard supplies a wide variety of key styles, grouped on the board to indicate different functional areas, such as cursor movement keys, numeric keys, function keys and keys such as space, shift, caps lock, all of which users are familiar with, and the functionality of which they are accustomed to. For instance, depression of the cursor movement keys results in repeated or rapid movement of the cursor in the appropriate direction, providing a convenient cursor movement method, with a minimum of effort (Shneiderman, 1998).

This level of sophistication will need to be applied to more intuitive input techniques (e.g. speech and gesture input, gaze-tracking, whole-body movement) and the associated hardware implementations (data-gloves, speech recognition systems, touchscreens, virtual reality body-suits) to provide the same level of acceptance, reliability and efficiency.

\subsubsection{Gestural Interfaces}

Studies of input devices have shown that users prefer certain types of gestural interface, over the standard mouse and keyboard combination. A study comparing the combination of hand-drawn gestures and handwriting, with the mouse and keyboard found that users were able to complete tasks more quickly using the gesture options against either using mouse and keyboard together, or mouse alone (Wolf, 1992). For the gesture options, a graphic tablet was used to perform the input. Early studies of handwriting recognition however, indicated that recognition accuracy would be the most significant factor in its acceptance and eventual integration into mainstream computing (Briggs et al, 1992; Mahach, 1989). In this study, a comparison of input through the mouse, alphabet keys, cursor keys and handwriting recognition, indicated that the handwriting was the least accurate of all the input methods. 
The general area of pen computing has developed through the introduction of new palmtop machines and electronic organisers, such as the Apple Newton and the 3COM PalmPilot ${ }^{\mathrm{TM}}$. Pen computing involves the elements of handwriting input, hand-drawn marks (which can be associated with commands) and the use of soft keyboards (a software generated keyboard rather than the actual device). Input is usually made with a stylus on an LCD display and digitiser, which allows precise selection and control of items on the display.

This method of interaction, using a pen on a surface, is similar to pen and paper, and is an attractive form of input, as it does not require users to learn new skills and has intuitive appeal (Wolf, 1992). Pen computing has had a significant role in applications such as air traffic control systems for several decades (Chatty \& Lecoanet, 1996; Mertz \& Lecoanet, 1997), and more recently, in engineering design applications (Zhao et al, 1995). However, the recognition accuracy of the handwriting input has constrained its widespread acceptance, especially for the input of Asian languages, where handwriting recognition is more complex. The $3 \mathrm{COM}$ PalmPilot ${ }^{\mathrm{TM}}$ has compensated for the accuracy problem by using a form of handwriting and gesture input called 'Grafitti', a stylised form of input made on the same area of the palmtop touchscreen. Each consecutive gesture is made over the last entry, and the performance and accuracy is reportedly high at $100 \%$ accuracy (3COM, 1998).

It has been noted that prolonged use of touchscreens and pen input can result in arm fatigue associated with raising the arm to point and select. The screen can also be obscured by the hand whilst entering input, and there were also problems with precision in the first widely available touchscreens (Shneiderman, 1998).

Touchpads have also been used, especially in notebook computers, as an alternative to trackballs, trackpoints and joysticks. As with touchscreens, the function of the mouse buttons can be simulated by tapping (single or double tap), or in the case of touchpads, with the presence of physical buttons near the touchpad, which can by operated by the index finger or thumb (Mackenzie \& Oniszczak, 1998). 


\subsubsection{Speech input}

There are now several speech recognition technologies available, with the most natural form of input provided by continuous voice recognition packages. In the past decade the capability of commercial voice recognition packages has improved, from constrained connected speech input, through discrete recognition systems, and finally to the provision of the continuous speech recognition software, which became commercially available last year. Most systems require that users have individual voice files, which they create by 'training' the software using provided vocabularies. The systems can also adapt over time to give a gradual increase in recognition performance, as the adaptation process 'learns' from the way individual users speak, dictate and pronounce.

An early example of connected speech input was used in the 'Put-that-there' study of the MIT Media Lab (Bolt, 1980; Bolt, 1984; Bolt, 1987 and Section 2.5.3.1). Connected speech involved several seconds of speech input, spoken naturally. The speech segment was then processed to determine a fixed number of words or utterances. This type of speech recognition typified the problems of processing human speech. Concepts such as co-articulation (where words in a stream would blend together, without clean boundaries) and segmentation (determining where the words start and stop) posed serious difficulties. The technological constraints of the day also limited the performance of speech recognition, where speaker-independent, continuous speech recognition was described as a 'formidable' task (Bolt, 1984).

Discrete recognition software performs voice recognition upon individual words, spoken with a slight pause between each word. Thus, each word is a 'discrete' utterance for recognition. Most systems are speaker dependent, where users are required to create vocabulary files, by repeating a selection from the overall vocabulary. Speaker independent recognition is also possible, but recognition rates are usually lower (Shneiderman, 1998). The size of vocabularies varies between individual commercial products, and is usually in the region of several thousand words (Fishman \& Padilla, 1997). Most discrete recognition 
applications provide the capability of commanding and controlling applications purely by speech, replacing the need for using the mouse and keyboard. Dictation can also be performed into text editors, although dictation into word processor applications (such as MS Word, v6.0) was not supported.

The portability and relative ease of use which the discrete recognition packages allowed, made them popular in particular situations, typically where hands-free control was required, or where speakers were involved in 'eyes-busy' tasks. Users ranging from baggage handlers to control inspectors have used speech recognition to reduce the 'writing down task' element of their work, when engaged in hands and eyes-busy occupations (DeHaemer et al, 1994; Shneiderman, 1998). There are also customised vocabularies available for specific professions such as lawyers, and medical specialities such as radiologists (Lai \& Vergo, 1997). Speech recognition technology has also had a significant impact on disabled users, who can gain mobility through the voice operated control of wheelchairs, and a wide variety of other equipment. 
Aside from the technological aspects of speech recognition, considerable care needs to be taken in the use of speech input to systems. As speech is used so readily by human beings, it may be assumed that the use of speech for human computer interaction would be beneficial, intuitive and easy to use (Nickerson, 1987). However, the design of any system needs to match speech input to suitable tasks, and be aware of the cognitive loads that may occur through the use of speech in some circumstances. Four main factors influencing speech interface design have been identified:

- identify the task types that may be executed more efficiently through speech input (DeHaemer et al, 1994)

- design a speech interface for improved user acceptance and efficiency (DeHaemer et al, 1994)

- $\quad$ provide adequate feedback so that users feel in control, and are able to correct errors (Sidhu \& Coyle, 1995)

- $\quad$ avoid complexity, which can strain users' memory, causing errors (Lai \& Vergo, 1997; Sidhu \& Coyle, 1995) (speaking commands is thought to be a more demanding task than those performed by the mouse (Shneiderman, 1998, p331))

While many studies of speech input indicate a user preference for speech input, some experiments have found that conventional means of input (mouse, keyboard) are more efficient, with lower error rates and task completion times (DeHaemer et al, 1994). This can be attributed, in part, to the unfamiliarity of users with the systems they are using (especially due to the lack of training in experimental situations), and the associated difficulty of remembering spoken commands (in vocabulary constrained or non-natural speech input systems) (Lai \& Vergo, 1997).

Continuous speech recognition software now supports dictation into a variety of applications such as word processing (Fishman \& Padilla, 1997; Shneiderman, 1998). The continuous speech recognition systems allow natural speech to be recognised, and although speaker independent recognition is possible, the speaker dependent approach provides more reliable and efficient recognition (by the creation of personal vocabulary files containing word and pronunciation models for individuals). The possibilities and capabilities of current systems is constantly 
improving. However, it will be the design considerations, and analyses of optimal ways to use speech input which will most likely drive progress in its use in computer systems. In particular, the possible roles of natural language and command and control strategies in our conventional desktop systems will be pivotal for progress (Cohen, 1992).

\subsection{Scope of thesis}

\subsubsection{Combination of input modes and redundancy}

The multiple ways in which humans can communicate includes verbal and nonverbal communication. Both verbal and non-verbal communication could be considered to encompass both vocal and non-vocal communication. Consider the following categories (Adler \& Rodman, 1988).

\begin{tabular}{lll}
\hline & Vocal Communication & Nonvocal communication \\
\hline Verbal Communication & Spoken Words & Written Words \\
Nonverbal communication & Tone of voice, sighs, screams, & Gestures, Movement, appearance, \\
& vocal qualities (loudness, pitch, & facial expression, etc. \\
& etc.) & \\
\hline
\end{tabular}

Figure 3: Types of Communication (taken from Adler \& Rodman, 1988)

In Figure 3, the definition of verbal communication implies the use of words and language. Non-verbal communication is any communication without the use of language (using the definition that language depends on the linguistic use of words, in contrast to naming gestures or posture as body language) (Adler \& Rodman, 1988). This is similar to the earlier definition from Frohlich about communication modes in human-computer systems, which he defined as language and action (Frohlich, 1991). The study of non-verbal communication also notes that it can be an ambiguous form of communication, and can be an explicit example of multiple channel communication, because there are so many forms of non-verbal communication which we can make.

Most non-verbal communication is performed by humans in tandem with verbal communication, and can fulfil a variety of functions including repetition, 
substitution, complementariness, accent, regulation, and contradiction (Adler \& Rodman, 1988). These functions are of interest to this thesis, as they highlight the possible information relationships that the use of multiple input modes in human-computer systems may provide.

The definition of repetition given by Adler \& Rodman (1988) is equivalent to our definition of redundancy, where equivalent information has been provided from distinct sources e.g. giving directions by speech ("Turn right at the next street, by the corner shop") and gesture (point to the shop). Substitution is the possible replacement of one channel of communication with another (a nod instead of a verbal "yes"). Complementing is information from another source which enhances or supports the main source in some way. Accenting (or emphasising) stresses content in another channel, while Regulation is the effect of non-verbal communication on verbal communication, through intonation and breath sounds, used to indicate possibly speaking will terminate. Contradiction is where two distinct channels contain conflicting information, providing an inconsistency between the channels.

These definitions match those outlined earlier by Basil (1994), (Section 2.5.7) on definitions of information overlap between communication modes.

\subsection{The Role of Information in Multimodal Input}

\subsubsection{Redundant and Complementary Information}

From these functions of non-verbal communication, two are of interest in this study of multimodal input: redundant and complementary information. One benefit of multiple input modes is the provision of additional information that the modes can supply. This additional information may be equivalent to, or different from, existing information in other modes. It may be redundant or it may be complementary. This distinction depends upon the input modes involved, the information quality of those modes (how successfully the information has been 
recognised and interpreted) and the distribution of semantic information across these modes.

\subsubsection{Choosing input modes}

The nature of the individual modes involved in multimodal input systems affects the degree to which information is redundant, complementary, enhancing or contradictory. For instance, a bimodal system with the input modes of speech and lip-reading will normally be expected to provide information on the same content - the spoken words. Thus, when both the speech and lip-reading input are interpreted, the information will be used for the same purpose, although the lipreading information may not be required if the speech recognition information is fully recognised.

In contrast, the input modes of speech and gesture have a more complex relationship with one another. They are independent of one another (the speech and lip-reading modes are dependent - lip-reading will not occur without speech). Therefore there are more possible information relationships between speech and gesture modes. Speech and gesture can be complementary to one another, they can contradict each another, or they can agree with one another (be redundant). This relationship, focusing on redundancy versus complementarity, between speech and gesture is discussed further in Section 3.5.

\subsubsection{Modal Quality}

If redundant information is present in one mode in a bimodal input system, and the information from that mode becomes corrupted through noise or some outside factor, then the previously redundant information may become critical for the resolution of meaning. In this situation previously unused redundant information, is no longer redundant, it may be the only source through which meaning can be resolved. 
Thus, in one sense, the term redundancy is incorrect, since the information becomes essential for further understanding. This was also noted by Cherry (1966), who referred to the term redundancy as a 'misnomer'.

\subsubsection{Semantic overlap}

The concept of redundant information therefore means that information is repeated in some way in another mode. All possible input information may be repeated between modes or only part of all possible information. For instance, a pointing gesture provides redundant information about the target pointed to in conjunction with speech about the same target.

With more than one input mode, the information carried in total by modes may not necessarily be redundant. The information could be contradictory, distinct (i.e. complementary), or some mixture of these properties. If there is partial redundancy in the system, then the information from one mode will be a subset of the other mode to some degree. Figure 4 shows examples of modal overlaps.

Example 1 shows complete redundancy between two input modes, as the information from one mode is also contained in the other mode. In example 2 , one mode is a strict subset of the other, it contains no other information distinct from the outer mode. The outer mode contains information not present in the inner mode. In example 3, part of the right-hand mode is a subset of the left-hand mode, while both modes contain information distinct from one another. Examples 4 and 5 are not redundant, showing contradiction and complementariness respectively. 


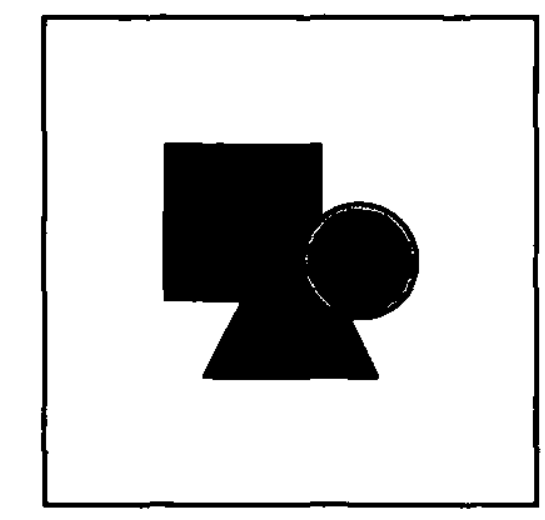

Figure 4: Diagram of Possible Modal Overlaps

$\square$ mode 1

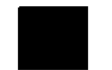

mode 2

Key:

- $\quad 1=$ complete overlap, redundancy between modes, equivalent information

- $\quad 2$ = subset overlap, redundancy, one mode a subset of the other

- 3 = partial overlap, partially redundant, partially complementary

- $4=$ no overlap, contradictory

- $5=$ no overlap, complementary, distinct information, not contradictory

We describe these concepts as semantic overlap in this thesis. This terminology has also been used by Vetere (1997) as 'semantic fit', where a highly positive semantic fit between modes implies repetition, and a highly negative semantic fit between modes implies contradiction. Vetere does not give further details of the intervals in between, although his discussion of general definitions in the area are helpful (Vetere, 1997). The semantic properties of repetition, substitution, augmentation, contradiction, or no relationship, are identified as common themes amongst available reports on the concept of redundancy (usually in noncomputing areas, see Basil, 1994; Lang, 1995 and Section 2.5.7).

\subsection{Complementary vs. Redundant}

The main contention of this thesis is that redundancy plays a major role in the interpretation of multimodal information and needs detailed investigation. However, there has been some recent research which argues for a change in 
emphasis towards complementarity in the area of speech and written modal combination.

Oviatt et al (1997) argue that spoken and written modes consistently supply complementary rather than redundant meaningful (semantic) information. Their research has provided empirical evidence about the integration of speech and written modes, as well as observations about temporal issues in integration, another area which has received little attention. Their work indicated that written input takes precedence over speech in multimodal interaction in $99 \%$ of cases where one mode of input occurred first (thus, sequential). Where input occurred in parallel (i.e. simultaneous), writing preceded speech in $57 \%$ of cases.

Others have argued that the information that gesture and speech convey is always complementary, and never redundant (Cassell et al, 1994). They argue that human gesture contains meaning not present in speech. They also discuss how gesture may be used to convey meaning which may be difficult to express in speech.

Complementary information may add meaning and may contain an essential element to facilitate understanding. Complementary information suggests information which expands and perhaps compensates other information sources. In contrast, redundant information suggests information which is not essential, and could be removed in certain cases (Cherry, 1966).

Cassell and Oviatt argue that if information is complementary, then it cannot be redundant (Cassell et al, 1994; Oviatt et al, 1997). However, the level of meaning at which this is applicable must be considered. For instance, if someone gives directions to a colleague's office, points up above and says 'He's upstairs', this combined action is redundant - people know that 'upstairs' is above, so the information 'above' has been received from two distinct sources. It could also be argued to be emphasis - if the person points first, before saying 'upstairs'. Therefore, information has been added from one channel which stresses the content of the other channel. As the emphasis adds information, it can be 
considered as a subset of complementariness. This example illustrates the difficulty involved in defining exactly what is considered to be redundant or complementary (Vetere, 1997).

Therefore, apparently complementary modes are not necessarily devoid of redundancy, especially when viewed in the context of gesture modes, as opposed to written modes. Clearly, the nature of the redundancy depends on the individual modes involved, the number of modes and the application dependent constraints. These factors determine application practicalities such as the mapping of tasks to modes, how tasks can be executed (via a combination of modes or through one mode only), and whether certain functionality is available through only one mode.

\subsection{Developing a framework}

A framework for investigating the role of redundancy in multimodal input has been used to develop an experimental system. The main requirement of an implemented system is the capability of probing the role of redundancy, through redundant input across two or more modes. Obviously, the system will need at least two input modes.

The chosen input modes are speech and two-dimensional gesture, as they provided a facility for varying redundancy in the combination of modes. Relatively simple input options have also been chosen, deliberately, for three reasons:

- We believe that redundancy can be exploited without the best possible quality of input recognition (the input modes of speech and two-dimensional gesture can be ambiguous and error-prone forms of input).

- Analysis of the information from these inputs and their processing requirements will be relatively simple.

- Any realistic implementation which is used widely will have to be inexpensive.

These factors will provide a basis for showing that redundancy alone can improve the input recognition task, without focusing on optimisation of input recognition and processing. 
In the multimodal system that we will develop, the information from the individual input modes will need to be combined into a multimodal representation. A consistent form of information representation will be required for speech, gesture and combined, multimodal information. Therefore, the type of information which will be generated from the separate speech and gesture recognition systems will dictate which information can be compared and combined to form multimodal representations.

\subsubsection{Speech input system chosen}

A commercial voice recognition system, and a software development kit are used for the speech input part of the multimodal system. The software development kit allows programming of an interface with a speech recognition capability and provides speaker-dependent and independent voice recognition (DragonVoiceTools Programmer's Guide, 1995). This speech input system was a relatively low cost option, and provided the required speech recognition capability. The system was the simplest available from the DragonDictate range, with a 5,000 word vocabulary provided as standard.

The speech recognition system has several options in the way it is used. Functions include:

- $\quad$ vocabulary training

- $\quad$ speech model adaptation

- language model adaptation

- vocabularies optimised for both low and high voices

The use of options such as vocabulary training and speaker adaptation increase the recognition rates for individual users of the system. Vocabulary training is the process whereby a user of the speech recognition system repeats a set of words. This process produces vocabulary (.voc) and user (.usr) files containing vocabulary information and the pronunciation information, respectively. The pronunciation information is the digitised speech model which is stored and is used for speaker-dependent recognition of that user's speech. Large speaker- 
- two-dimensional gesture recognition is a simpler problem

- real-time processing of three-dimensional gestures is more difficult to achieve than two-dimensional gestures

- three-dimensional gesture recognition has greater processing and hardware requirements than two-dimensional gestures

The implementation and integration of a three-dimensional gesture recognition system into a final multimodal system would have greater processing and hardware requirements than a two-dimensional gesture recognition system. Three-dimensional gesture recognition systems for gestures such as pointing, finger, hand, arm and body movement, facial expressions, and eye movement all require movement capture devices. These include tracking movement through observation with video cameras, and the use of datagloves which use transducers and attached sensors to monitor and record the gestures. Algorithms are then required for processing the gesture data, and classifying into appropriate categories. This area is currently receiving significant attention, and approaches for optimising the recognition and classification strategies are appearing (Wachsmuth \& Frohlich, 1997). By comparison, the problem of two-dimensional gesture recognition is simpler since both the gesture capture and processing are more easily accomplished with current technology. 


\section{Chapter 4}

\section{Initial Development}

\subsection{Introduction}

This chapter describes how the gesture recognition system was implemented and, in particular, gives details of the parameters used in the multimodal recognition process. Initial performance results from this gesture recognition system are presented. Preliminary ideas for the investigation of the speech system are also described.

\subsection{Motivation}

An initial motivation in the development of the gesture recognition system was simplicity. We wanted to design a simple gesture recogniser, which would characterise gestures as a set of information parameters which could be used in further analysis. These information parameters needed to be detailed enough to provide distinguishability between distinct gestures, and general enough to be capable of describing each of the gestures. (This approach is similar to feature 
detection and extraction, used in pattern recognition and image classification (James, 1987)). With these key information parameters from the gesture recognition system, and similar information parameters accessible from the speech recognition system, a system could be created whereby two independent sets of parameters would be available for analysis each time a multimodal action occurred.

The initial aim was to produce as many specific information parameters as possible from the given input in order to provide the analysis process with a rich data set. Other factors which affected the design of the recogniser were software and hardware constraints. The speech recognition software chosen was only available on a PC platform running MS Windows 3.1, so the gesture recogniser would have to be compatible with this software and would therefore be platform dependent. As a consequence, it was decided to develop a simple system, using the $\mathrm{C}$ programming language.

\subsection{Characteristics of $2 D$ Gestures}

Gestures, particularly two dimensional gestures, are inherently command-based. Whether considering circles, crosses, arrows etc., each can easily be mapped to a command (Bordegoni \& Faconti, 1996). Proof readers and editors demonstrate the use of such two-dimensional gestures or editing marks. Kramer has noted their command based nature and identified approaches for exploiting these properties (Kramer, 1997). Their command nature is different from that of a command provided through a pop-up or pull-down menu. Unlike a menu command selection, gesture command can, directly and in parallel, specify the operand (or target) of a command through its spatial information content.

Two dimensional gestures have been classified with a set of properties which belong to seven distinct categories: input device, display vs. input, content vs. gestures, segmentation, command execution, visualisation, and interpretation (Kramer, 1997). 


\subsubsection{Input device}

In the category of input device, Kramer (1997) describes the variety of possible ways that two-dimensional gestures can be created. He lists a pen, pen with modifiers, finger, mouse and multi-modal methods, describing how pen input may involve the contact of a pen on a surface, or the proximity of a pen to a surface (some digitiser surfaces sense proximity). Pen with modifiers, refer to mechanisms integrated with the pen which change the state of the pen in some way, usually to differentiate between the entry of commands and content marks (such as handwriting). Finger input is the use of the finger on a touch sensitive screen or touchpad, and mouse covers the creation of gestures by manipulating the cursor. He describes multimodal gesture input as the combinations of these input methods.

In our implemented system, input is made with either a pen stylus or finger, on a touch sensitive screen. The pen does not possess modifiers of any type, although modification of the 'state' of the touchscreen is possible, allowing changes to the button mode (changing mapping of touches from left to right mouse button presses etc.) to be made.

\subsubsection{Display vs. Input}

The type of surface on to which the input can be made is divided into two categories, display and input. As seen in earlier gestural studies (Section 2.5.5.1), the input surface and display may be equivalent, or they may be distinct. For instance, touch sensitive screens attached to the display monitor allow input 'on' to the display surface, as do personal digital assistants. In contrast, touchpads, the mouse, digitisers and graphic tablets all make input on a surface separate from the display.

Using the same input surface and display allows immediate feedback of input gestures to users. On distinct surfaces users may have to move their gaze from the input surface to the display, which distracts them from the input task. One disadvantage of coupled display and input systems however, is that input such as 
handwriting and manipulation of menus, can be obscured by having the hand, pen or finger on the display. In our system, a pressure sensitive touchscreen is attached to the display monitor, providing the display and input of gestures on the same surface.

\subsubsection{Content vs. Gestures}

Kramer classifies two-dimensional gesture input as either containing content for the interaction (e.g. handwriting), or a gesture command. For instance, the entry of a zigzag stroke could represent a delete gesture, if drawn on an object, but could also represent the letter $Z$, if drawn on white space (Kramer, 1997). Systems can be classified according to the way in which they cope with these possibilities. Some systems operate on the assumption that all gestures are command based, and contain no content, while others operate in reverse, assuming all marks contain content and not command marks. Some systems allow both types of gesture, with the context of where the gestures are entered determining how the gesture is interpreted. There are also more explicit methods for determining which interpretation of gestures will occur. This can be through a device (a button) on the pen stylus which allows easy switching from gesture to content mark ('lightweight modes'), or a more permanent switching of context may occur through a specific button press or gesture mark ('heavyweight modes').

As all two-dimensional gestures in our system are command based, and no content marks such as handwriting input are made, interpretation between these possibilities was not necessary.

\subsubsection{Segmentation}

Segmentation is the problem of deciding when a gesture begins and ends, and is required for the interpretation of the gesture. For instance, if a system contains compound gestures (combinations of other gestures) the system needs to make a decision about when a gesture is a single gesture, or the beginning of a compound 
gesture (compare an arrow $\rightarrow$ which consists of two gestures, with a line -, and the end angle $>$ ). There are several approaches to segmentation analysis:

- $\quad$ all gestures are assumed to be single strokes;

- no compound gestures are allowed in the gesture set;

- a delay between gestures indicates that they are separate gestures;

- if the time between one gesture and the next gesture is outside a specific time gap, they are regarded as separate gestures;

- the context of gesture marks determines whether a gesture is considered complete.

In our system a mixture of these properties is used. Most gestures are single strokes, with the exception of the cross gesture, which is a compound of two line gestures (see Section 4.4.1). Therefore, all strokes apart from the cross constituents are regarded as single strokes. When a line is gestured, if another line follows within a specific amount of time, it is interpreted as the second segment of a cross.

\subsubsection{Command Execution}

For two-dimensional gestures that are used for command input, the question of when to execute these commands arises. There are three basic possibilities, immediate, direct and deferred. With immediate execution, a gesture is executed whilst it is still being entered (for example, in drag-and-drop and text selection). Direct execution performs a command after it has been recognised, whilst deferred execution performs the command when users indicate this.

In our initial gesture recognition system, the deferred command execution approach is used. Users indicate that the gestures are complete by depressing a 'send' button. This was modified in the final implemented gesture system to the direct execution approach, where the gesture was executed after it had been recognised. In the final multimodal system, command execution depended on both speech and gesture modes, and was a variation on the direct execution approach. 


\subsubsection{Visualisation}

Visualisation of gestures describes the possible feedback that is given to users upon entering a gesture. Entered gestures may be invisible, such as certain scroll gestures, where no trace of the gesture is made on the display. Other gestures may have their trace drawn on the screen. Feedback on some gestures is indicated at the location of the gesture, during the gesture execution (e.g. dragand-drop operations where a file icon is moved with the path of the gesture).

Since it is important to analyse the gestures made and compare them with those the system has recognised, our system uses the trace style of visualisation.

\subsubsection{Interpretation}

Finally, the mapping of gestures to commands, can vary according to the application requirements. Some systems employ fixed gestures, which are mapped to the same commands throughout the application, while other systems may have a variation in the mappings according to either context or user choice. (see Content vs. gestures section above).

Our final gesture system uses fixed mapping of gestures to commands, where crosses represented cut commands, a circle represented a copy command, a tick gesture represented paste commands, and lines represented move commands. Also, as two-dimensional gesture input is ideally suited for direct manipulation, gestures can also implicitly contain object and location information in addition to command mapping.

\subsection{Gesture Recognition System Development}

Two-dimensional gesture recognition systems are mostly found in applications for pen computing, in personal digital assistants and palmtops etc., and especially in air traffic control systems (see Chatty \& Lecoanet, 1996 and Section 3.2). These types of recognition systems are more rare in conventional desktop computer systems, however. The most well known two-dimensional gesture 
recognition system is probably the work of Rubine (1991), who devised a trainable gesture recogniser for single stroke gestures. The system uses vectors of gesture features to classify families of gestures, and uses analysis of angles between certain points of gestures, calculating sine, cosine and tangent values for further feature extraction (see Section 2.5.5.1 for further discussion of Rubine's work). However, the system does not process multiple gestures.

The gesture recognition system implemented for this research, is deliberately basic and simple. The method of identifying gestures is very similar to the feature extraction approach, used in pattern recognition, in terms of how certain features or characteristics are measured in the input data (James, 1987; Tou \& Gonzalez, 1974).

\subsubsection{Initial gesture recognition system}

Two-dimensional gesture input was made through a pressure-sensitive touchscreen mounted on the computer monitor. Gestures could be made with the finger or with a simple pen dragged across the surface of the touchscreen, and the path of the gesture is traced out on screen. The system processes all the coordinates of the gesture, made by the user.

The initial gesture recognition system only used two tests to differentiate between two gestures, a tick and a circle. A tick was classified by the change of gradient which occurs in that gesture, and a circle was classified by its start and endpoints being relatively close together.

Early experimentation with the system indicated that there were a number of factors which could affect recognition when input was made with the touchscreen. Gestures which we would visually classify as ticks were rejected, when the relevant change of gradient was clearly present (Figure 5 and 6). This was caused by wobbles and jerks which can arise when too much pressure is applied to the touchscreen, as the wobble or irregularity is picked up by the system, rather than the relevant gradient change of interest. 


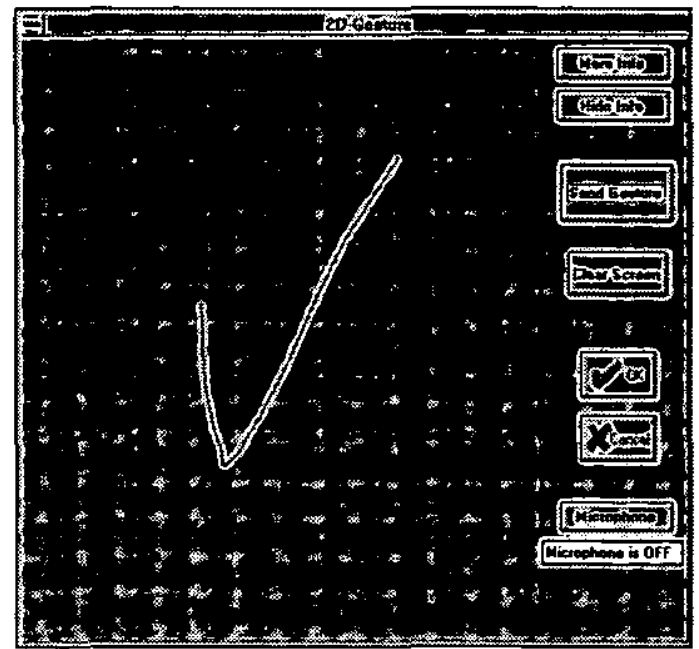

Figure 5: A simple tick gesture

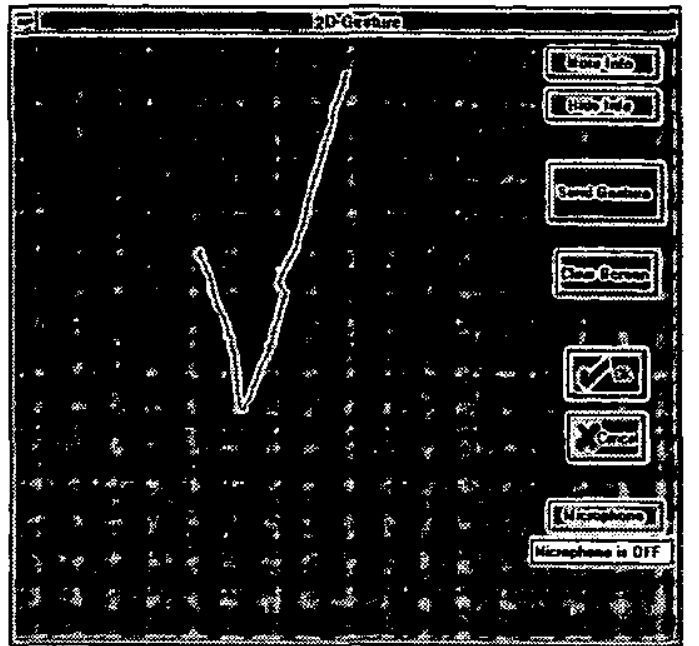

Figure 6: An irregular tick

This factor had considerable effect later when recognition statistics were examined (see Section 4.4.2).

Following the initial examination of the two gestures, ticks and circles, the scope was broadened to include more gesture types. The set of possible gestures was expanded to include circles, ticks, crosses, and lines (vertical, horizontal, positive and negative gradients). The gesture input was analysed for a selection of geometrical patterns, to enable a classification of the gestures to be made. The patterns and percentages of gradients in the co-ordinates produced by the gesture were analysed to calculate statistics about line content, gradient changes through the gesture, intersections between multiple gestures and gestures which selfintersect.

Further attributes including area, duration of gesture, time-stamps and measurements of the distance between start and endpoints of gestures were also calculated. This approach of searching for line content in gestures, is similar to the basics of syntactic pattern recognition, where the structure of patterns is used in the recognition process. In the case of gestures, the components of the gestures are line segments of varying length, which have distinct relationships for each gesture. In syntactic pattern recognition, the inter-relationships of components of a pattern are used to aid in the recognition process (Tou \& Gonzalez. 1974). 
From the co-ordinates received by the touchscreen, two sets of gradients were calculated, a gradient between every point of the gesture and a gradient between six points of the gesture. These gradients were then used in calculations of a set of parameters, shown in Table 4 .

\begin{tabular}{|c|c|c|}
\hline Gesture Test & Purpose & Result \\
\hline $\begin{array}{l}\text { Negative to positive } \\
\text { gradient test }\end{array}$ & Tests for a change in gradient from negative to positive & true or false \\
\hline Frequent gradient test & Tests for frequent changes in gradient & true or false \\
\hline Endpoint test & $\begin{array}{l}\text { Tests whether start and end points for the gesture were } \\
\text { near to one another or far apart }\end{array}$ & true or false \\
\hline Duration test & Calculates how long the gesture took (in mSecs) & time in mSecs \\
\hline Vertical test & Calculates the vertical line content & vertical gradient $\%$ \\
\hline Horizontal test & Calculates the horizontal line content & horizontal gradient $\%$ \\
\hline LineSlope test & $\begin{array}{l}\text { Calculates how much of gesture had significant sloped } \\
\text { line content (above } 50 \% \text { of gesture) }\end{array}$ & $\%$ of gesture \\
\hline $\begin{array}{l}\text { Positive and Negative } \\
\text { gradient test }\end{array}$ & $\begin{array}{l}\text { returns the percentages of negative and positive gradients } \\
\text { in a gesture }\end{array}$ & $\begin{array}{l}\text { negative \& positive } \\
\text { gradient } \%\end{array}$ \\
\hline Calculate_area & Calculates the area of closed or nearly closed curves & $\begin{array}{l}\text { area measurement } \\
\text { (pixels }^{2} \text { ) }\end{array}$ \\
\hline Intersection test & $\begin{array}{l}\text { Tests to see whether the strokes of a complex gesture } \\
\text { intersected one another }\end{array}$ & true or false \\
\hline Self Intersect test & $\begin{array}{l}\text { Tests to see whether a simple gesture intersected itself } \\
\text { e.g. a closed circle }\end{array}$ & true or false \\
\hline Area test & $\begin{array}{l}\text { determines whether to call calculate_area for a gesture } \\
\text { based on the value of Self_intersect test }\end{array}$ & true or false \\
\hline $\begin{array}{l}\text { Length of time between } \\
\text { current and previous } \\
\text { gesture }\end{array}$ & $\begin{array}{l}\text { calculates the time between the end of the previous } \\
\text { gesture and the beginning of the current gesture }\end{array}$ & time in mSecs \\
\hline Total points & $\begin{array}{l}\text { calculates the total number of points contained in the } \\
\text { gesture }\end{array}$ & number of points \\
\hline
\end{tabular}

\section{Table 4: Calculated gesture parameters}

Most of these heuristic tests arose from observing the performance of the system, during gesturing. The pattern of gradient changes was monitored and estimates of average points in each gesture was made. The test for a change in gradient from negative to positive was required to detect the shape of a tick gesture, where the shorter down stroke is usually of negative gradient ( ), and the longer up stroke is usually of positive gradient (/). The test for the frequent changes in gradient was used to identify possible circle gestures, as could the endpoint test, which calculated the distance between begin and end points of gestures. If this value was above a set level, the possibility of the gesture being a circle was rejected, and if the value was below this level, a circle gesture was a possibility. The threshold level was determined by observation of a set of circle gestures, by measuring the distance on average between the start and endpoints. 
The duration test returns the length of time taken to execute the gesture. This figure varies with each gesture, as line gestures take less time to perform than circle or tick gestures. The vertical and horizontal tests are mostly used in the identification of vertical and horizontal lines. These tests return the percentage of vertical and horizontal gradients in each gesture. The horizontal test is also applied to the identification of circle gestures, which were observed to contain a significant percentage of horizontal gradients, although a lesser amount than a horizontal line. The line-slope test calculates whether the amount of positive or negative gradient percentages in a gesture are above a particular threshold, which had been chosen as the significant level above which most line gestures of positive and negative gradients occurred.

The test for both positive and negative gradients in one gesture helps in the identification of tick gestures, which have particular levels of both these gradients. The area tests are used in the classification of circle gestures. The selfintersect test is another aid in the identification of circles, as this test determines whether a gesture has intersected with itself at any point. This is a common property of circle gestures, and not related to any of the other gestures.

A set of seven combinations of the tests were used. These heuristic combinations, based on experimental observation of the gestures made using the system, were used to decide upon the type of gesture made. 
The tests were of the following form:-

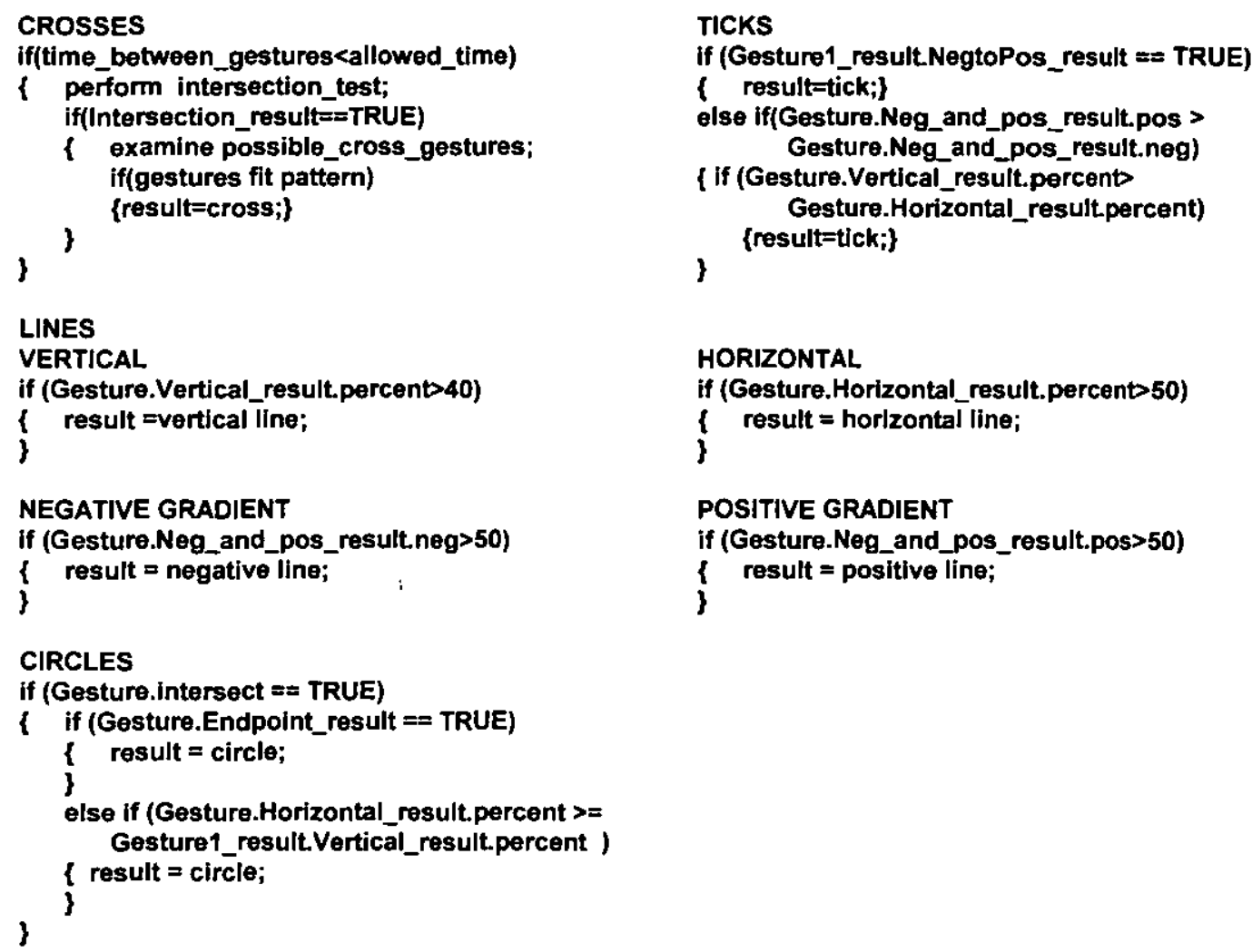

\section{Figure 7: Example of test combinations for gestures}

In the recognition process, after the gesture parameters had been calculated for the current gesture, these rules were applied to the parameters. The classification procedure returned one gesture conclusion for each gesture made, testing for each gesture type in sequence. If one combination of tests failed, the next combination was tried until a combination returned a positive identification of a gesture. If all the test combinations failed, a don't know conclusion was returned for the gesture. This was a simple sequential process, where the gesture tests were independent of one another. The gesture parameters were used in each test, and this was the only information common to them.

In preliminary experimental observations, the recognition system produced around $90 \%$ recognition success figures for line gestures (vertical, horizontal, negative and positive gradients), and lower rates for the more complex gestures such as circles, ticks and crosses (around 50\%). 
In particular, recognition of a cross gesture was more problematic than the simpler line gestures. A cross is considered to be a complex gesture as it has two component gestures in this system, a line of negative gradient and a line of positive gradient. Their intersection is necessary for the gesture to be considered to be a cross. The combination of negative and positive line gestures in contrast to vertical and horizontal lines was chosen, as the negative and positive gradient combination had a much higher recognition rate for the intersection test in preliminary trials.

There were also difficulties associated with finding the intersection of a vertical and a horizontal line as a true vertical line had to be classified specially since its correct gradient is equivalent to infinity, rather than a tangible integer value. The problem of infinite gradient could have been compensated for with the use of polar co-ordinates rather than Cartesian co-ordinates, (where $x=r \cos \theta$, and $y=r \sin \theta$, for a vector $\underline{\mathbf{r}}$ with angle $\theta$ between $\underline{\mathbf{r}}$ and $\mathrm{x}$-axis). However, it was decided to remain consistent in the treatment of all gestures, and constrain the processing to Cartesian co-ordinates.

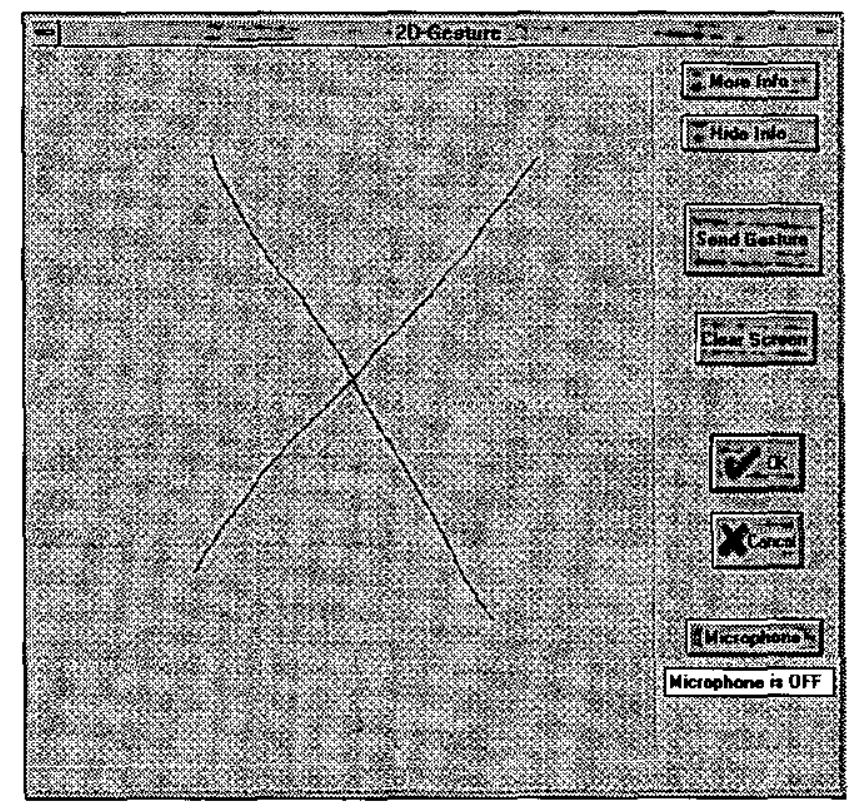

Figure 8: A complex gesture, a cross 
Rubine (1991) did not include complex gestures in his trainable gesture recogniser due to temporal problems of recognition, such as which two gestures belong together i.e. the segmentation problem (Section 4.3.4). In the early gesture demonstrator, where output of a cross is shown in Figure 8, the gesture was not sent for recognition consideration until the user had depressed the 'Send Gesture' button. This avoided the segmentation problem in the early systems, by using the user deferred execution style.

\subsubsection{Initial Results}

The initial two-dimensional gesture recognition system was implemented, based on the initial gesture recogniser. Possible gestures detected included: circle, tick, cross, or lines (vertical, horizontal, positive and negative gradients). Some preliminary tests were performed using the system, first using the touchscreen to make gestures, and then comparing these with gestures made using the mouse. This revealed a common problem connected with touchscreens as an input method: the precision quality of input can be poor (Shneiderman, 1998). 
It was observed that the quality of gesture input was affected by the input medium used. Irregularities in the gesture input occurred when using the touchscreen, in contrast to the mouse. Mouse created gestures were more precise in comparison to the gestures made using the touchscreen's 'fuzzy' input. Note the difference in the diagrams below. Figure 9 shows gestures made with the touchscreen, with detected self-intersections plotted (as the symbol $\nwarrow$ where the self-intersection was detected). These were plotted for information and program debugging purposes. Figure 10 shows gestures made with the mouse, with the selfintersection plot also in operation, although as can be seen, no self-intersects were detected in the mouse created gestures. Results in Table 5 also show the differences between the two associated streams of data for the twenty ticks shown on each screen.

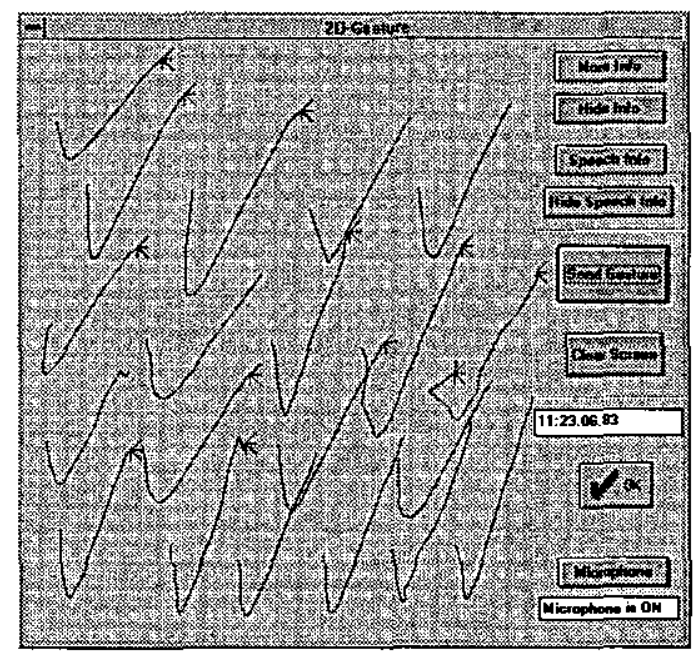

Figure 9: Tick gestures made using touchscreen

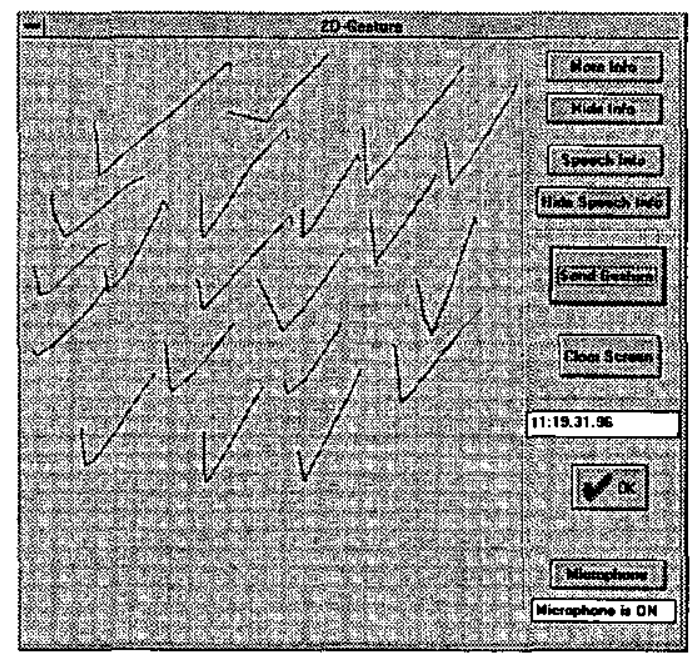

Figure 10: Tick gestures made using mouse

\begin{tabular}{llllll}
\hline Gesture & $\begin{array}{l}{ }^{1} \text { Positive }>= \\
\text { Negative }\end{array}$ & $\begin{array}{l}{ }^{2} \text { Vertical } \\
>=\text { Horizontal }\end{array}$ & $\begin{array}{l}{ }^{3} \text { Gradient } \\
\text { change found? }\end{array}$ & $\begin{array}{l}{ }^{4} \text { Line } \\
\text { slope } \\
\text { detected? }\end{array}$ & $\begin{array}{l}{ }^{5} \text { Self- } \\
\text { intersect } \\
\text { found? }\end{array}$ \\
\hline $\begin{array}{l}\text { Tick (by hand, } \\
\begin{array}{l}\text { Figure 2) } \\
\text { Tick (by mouse, } \\
\text { Figure 3) }\end{array}\end{array}$ & $100 \%$ & $100 \%$ & $25 \%$ & $50 \%$ & $55 \%$ \\
\hline
\end{tabular}

Table 5: Percentage of gestures matching criteria, 
KEY:

$1: \%$ of gestures with positive gradient content greater than negative gradient content

2: \% of gestures with vertical gradient content greater than horizontal gradient content

$3: \%$ of gestures where negative to positive gradient change found

4: $\%$ of gestures where significant sloped line content found

5: \% of gestures where a self-intersection has been found and plotted on-screen

Table 5 shows that the various gesture recognition tests have varying success dependent upon the medium used. For instance, ticks can be gestured which human beings clearly recognise as a tick, regardless of the medium used, but in which the gesture recogniser finds significantly differing characteristics. The medium used (touchscreen or mouse) is clearly responsible for many of these recognition variances. It is interesting to note that no self-intersections are found in the gestures made by the mouse whereas a high percentage of self-intersections are found in the gestures made by finger.

This difference is explained by the pressure exerted on the touchscreen during gesturing with the finger. The pressure is detected by the touchscreen and it attempts to plot the co-ordinates at the point where pressure has been detected. This can result in many points being detected by the system in a relatively small area of the screen (5-10 pixels wide). These points, when linked into a flowing gesture, are interpreted by the system as forming intersecting lines, therefore returning a positive result when the self-intersect test is performed. Obviously this pressure element is not present when the mouse is used.

The media used (touchscreen and mouse) have different properties (for example, method of input, rate and accuracy of data receipt) and it is this which perturbs the simple gesture recognition. The chosen solution for the two observed modally overlapping media (touchscreen and mouse) is to add further heuristic tests which desensitise the gesture recognition routine. This can be achieved because the two media have significant, but not extremely different, performance parameters. Perhaps, if performance parameters had differed significantly, two distinct recognition routines would have been necessary. This is perhaps a sub-set of a much larger issue of examining the implications of media with overlapping modalities. 


\subsection{Speech Recognition}

\subsubsection{Introduction}

To develop a system which incorporates a speech recognition element, it was necessary to have a speech recognition development environment. This provided access to the $\mathrm{C}$ programming language functions, which control the speech recognition elements and components.

This research makes use of the software developer's kit for the DragonDictate discrete voice recognition software. The software performs recognition on the analogue signal received from the microphone and returns one or more recognition results. The recognition results can be accessed through the software development kit, which allows manipulation of other recognition parameters such as:

- number of recognition results to return

- recognition level cut-off value

- percentage confidence values for the recognition results

\subsubsection{Developing Speech Aware Applications}

To provide a speech recognition capability for an application, three main decisions were required:

- to decide upon the use of speech recognition options (e.g. use the adaptation option, use the language model option, which parameters to adapt and change, use speaker-dependent or speaker-independent options)

- to decide upon the application vocabulary (e.g. use a specific vocabulary, use the provided vocabulary, use a combination of both)

- $\quad$ to decide upon the format of speech input (e.g. constrained with the program controlling the state of the microphone, or unconstrained with the microphone constantly on)

Speech recognition could occur without any prior vocabulary training, although recognition rates would be lower than if training had occurred. Use of the adaptation and language model options can increase the recognition rates. The adaptation option will automatically adapt and improve the stored speech models 
in a user file, providing improved recognition on future use of the system by that user. The language model stores information about words in a vocabulary, including their expected occurrence frequency, and information on other words which may occur with that word in speech.

Use of the adaptation option is only relevant if speaker-dependent recognition is being performed, where a vocabulary file containing the pronunciation information for individual users is stored. These files need to be loaded into memory before word recognition can occur. Some initial use of the software by users has to be performed to create personal vocabularies, and this involves the repetition of a selection of words associated with the application, to create vocabulary files containing phonetic (digital) word models of the speech provided. Speaker-independent recognition uses large scale provided vocabularies, which have been created by sampling the speech of hundreds of English speakers. Two versions of these vocabularies exist, one optimised for speakers with high voices, and one optimised for speakers with low voices.

A speech-aware application can use the speaker-independent vocabularies and create a specialised vocabulary which can be referenced in the recognition process.

\subsubsection{The process of recognising speech}

The Dragon system performed speech recognition in several steps. Speech input is made through a microphone, worn close to the mouth of the speaker (about 1 inch from the corner of the mouth). This speech is captured by a sound card, which produces a digitised model of the speech. The process from speech utterance to word recognition is shown in Figure 11. 
3-10 Speech Processing with the Dragon Speech Driver

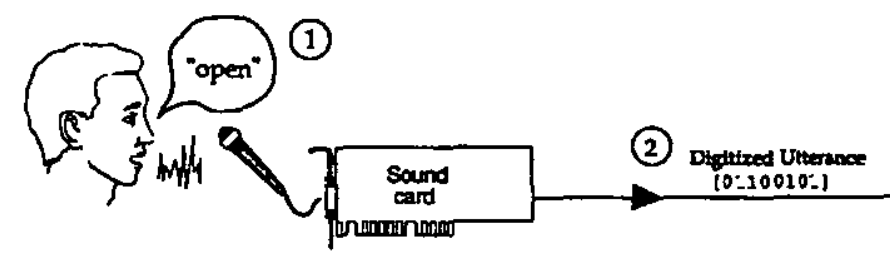

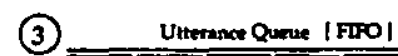
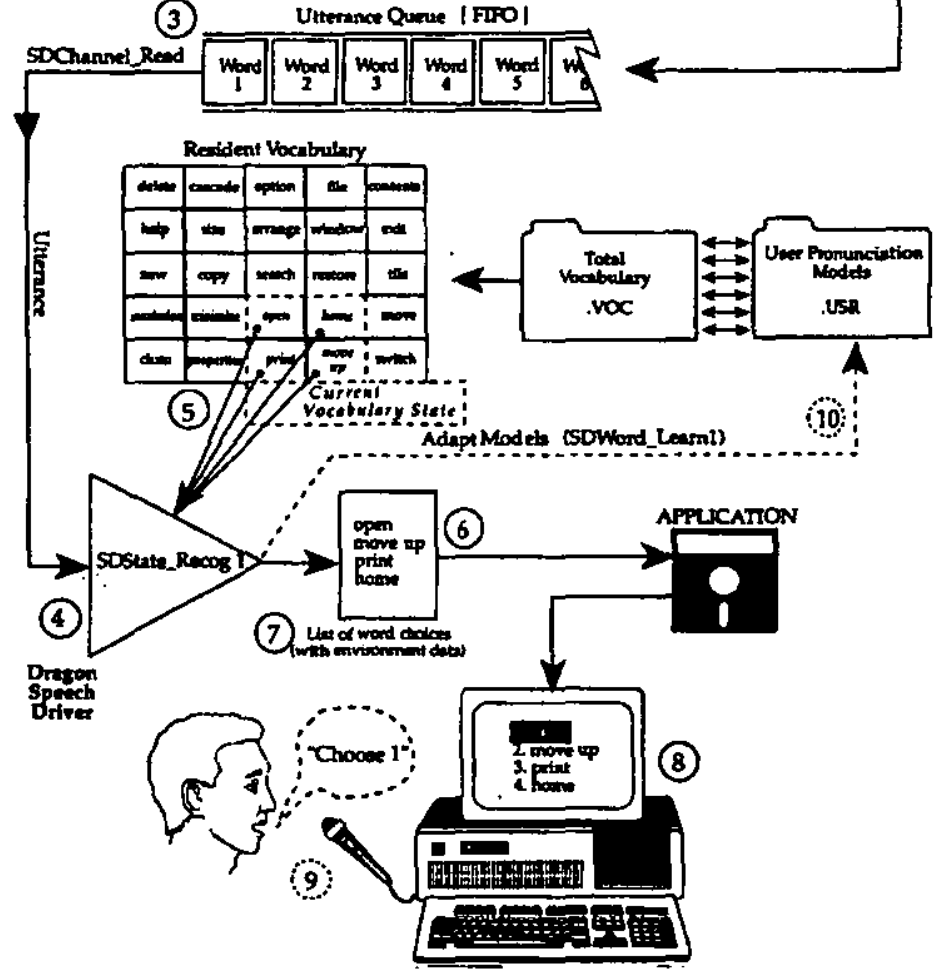

Figure 11: Speech recognition process in the Dragon ${ }^{\mathrm{TM}}$ system

The digitised speech is held in a buffer, and is processed in order by the recognition software. Consulting the resident vocabulary and user files containing the vocabulary information and pronunciation models, the system provides a set of recognition possibilities which the utterance matches to a degree. The number of possibilities provided depends on the options set in the system, the confidence value at which possibilities are rejected, and the maximum number of possibilities to be returned. The system also presents confidence values and 
distance values for these recognition possibilities, and if appropriate, environment data about the choices stored in the language model.

The application to which the recognition possibilities are sent provides the framework for deciding which possibility will be chosen. The choice may be made by presenting the user of the application with the choice, or the decision may be made by the system.

\subsubsection{Speech input system developed}

The development of the speech input component, for use in the final multimodal system, involved programming of the software components, including standard personal vocabulary and user file creation, choice of low or high voice speakerindependent vocabularies, and implementation of the software functions to provide speech recognition capability to an application.

Several initial decisions were made about the use of the speech system.

- Individual vocabulary files would be created for each user of the system, and would be used in addition to the speaker-independent vocabularies provided. This strategy increases the recognition rate for once-only users of the system, and requires an initial training period for users, where repetition of a limited vocabulary is required.

- The adaptation option was not used. The purpose of adaptation is to improve the stored speech models for individual users over extended use of the speech system (DragonVoiceTools Guide, 1995). Users of this system, the subjects in the experiments, would not be making use of the system in this way. Since on-going adaptation may increase the speech recognition rates through the duration of experiments, this would complicate the recognition results, as there was no equivalent adaptation for the gesture recognition system. Therefore, adaptation was not relevant for our purposes.

- The option of language models was not be used, as the effect of using this extra information in the recognition, while probably improving speech recognition rates (DragonVoiceTools, 1995), would have reduced the scope of recognition possibilities. This would defeat the aim of our input design, of providing a rich set of information possibilities and choices.

Based on these decisions, users were asked to repeat fourteen words, seven numbers and the sequence alpha, bravo, charlie, delta, foxtrot, golf (Figure 12) in 
the developed speech input application (which would be used in the final multimodal system).

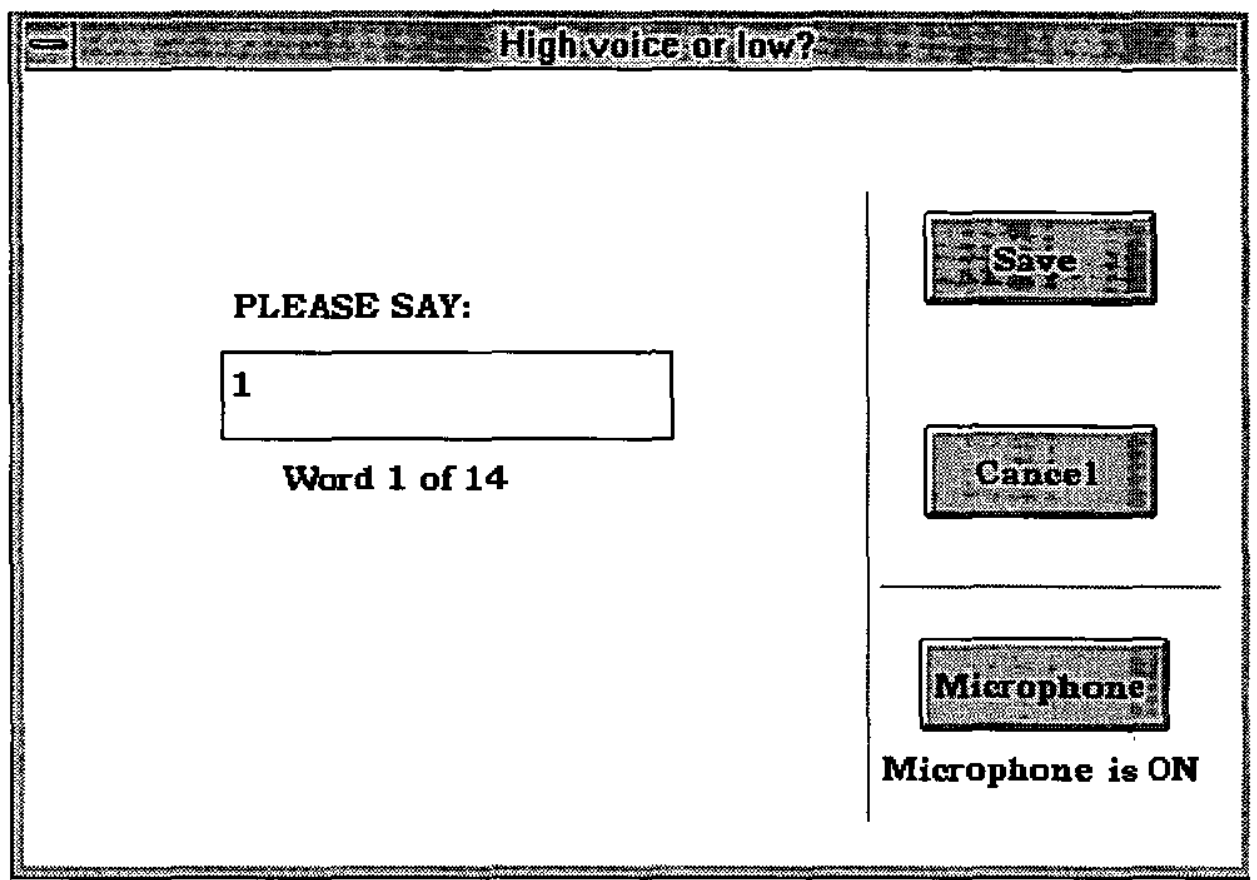

Figure 12: Choosing high or low vocabularies based on utterances

These utterances were then analysed by the system and matched to either high voice patterns or low voice patterns. After this match had been performed, all recognition from the speaker-independent vocabularies was then referred to the chosen high or low voice vocabulary. A further training session was then required to produce individual vocabulary and user files. Users were asked to repeat a selection of application dependent words, which included the final task vocabulary (Figure 13). 


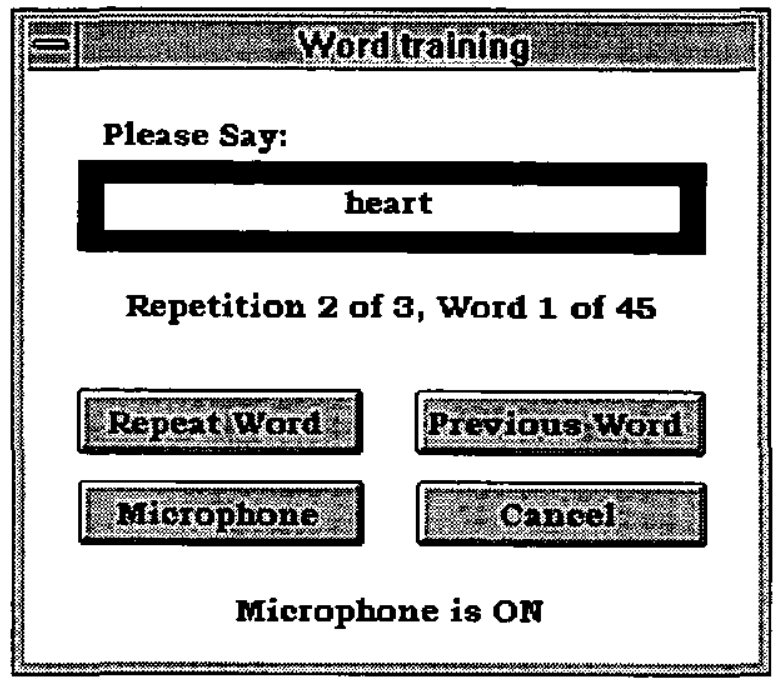

Figure 13: Vocabulary training window

The speech recognition system provides input information parameters. Key parameters are

- the word recognised

- a percentage confidence rating about the word

- duration of the utterance

These parameters are provided directly by the speech recognition software. The confidence rating is an arbitrary figure. It is not an absolute or constant rating and cannot be used to compare recognition efficiency between, for example, different vocabularies. Other useful information provided about the speech input includes:

- other possible word choices from the recognition process

- $\quad$ context of the word

- number of phonemes

- $\quad$ recognised phonemes

The last two of these possibilities although possibly interesting proved inaccessible. This type of information is part of the programming 'stack' several layers down in the Dragon system. This is accessed through the proprietary code and was therefore not available. These information parameters were therefore discounted. 
The parameters that were chosen to characterise speech input, for use later in the multimodal integration phase, were therefore:

- word recognised

- percentage confidence about this choice

- other recognition possibilities for this word

- values representing how 'correct' these choices may be

- value at which possibilities are rejected

Simple feedback was provided in applications about the first choice recognition possibility, to confirm that speech input had been received at the microphone and processed by the system.

Feedback window showing first recognition choice of 'copy'. Window below shows previous word recognised (no previous word spoken in this case)

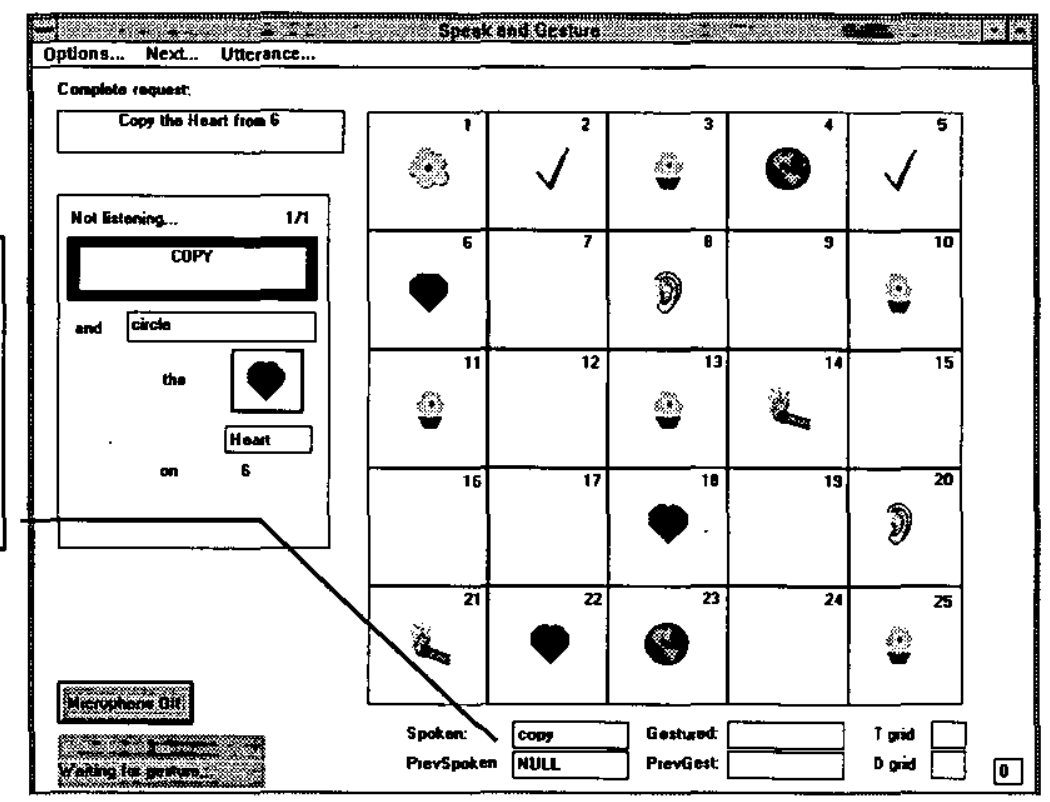

Figure 14: Speech feedback detail of application window

The format of speech input was constrained, so that comparison of intended with recognised speech input could be made. Although constrained speech input is restrictive for users, the experimental aims required that interpreted speech input was provided to the system. Natural, unconstrained speech input would have created complications in the handling of the speech input, and was beyond the scope of the experiments. 


\section{Chapter 5}

\section{Gesture Experimentation}

\subsection{Introduction}

This chapter describes the initial experimentation, which was performed using the two dimensional gesture recognition system, introduced in the previous chapter. The motivation for experimentation is outlined, and the experimental format is presented. A rule induction technique for classifying the gesture data is introduced, together with discussion of the results of the gesture experimentation. The use of production rules from the use of rule induction, in a truth maintenance system within a blackboard architecture is discussed. The application of the truth maintained blackboard system within the final gesture recognition system is presented.

\subsection{Motivation}

The aim of this set of gesture experiments was to test the robustness of the developed system, and to gain an understanding of its gesture recognition 
performance. The results obtained are used to design the final multimodal system. The two-dimensional gesture data produced from the subjects, are used to induce general gesture rules, in order to generate classification models for the gestures. As the gestures were obtained from a varied set of users, who each performed their gestures in an individual way, the induced rules were expected to be representative descriptions of all possible gestures in the set.

\subsection{Experimental Format}

In order to compare gestures made by subjects with the gestures actually recognised by the system, it was necessary to note which gestures users were attempting to make, and relate these to the gestures the system had recognised. Automatic logging of the attempted gestures was therefore implemented.

The task for the gesture experimentation also needed consideration. Early prototypes had involved the movement of coloured shapes from one part of a screen to another, using gestures in a simple shape manipulation application. This idea of manipulating shapes was developed into an application which involved the cutting, copying, pasting and moving of icons, around a numbered grid (Figure 15), by the use of two-dimensional gestures. This task allows the gesture recognition system to be tested for command, object, and location interpretation in an application. (The use of the terms cut, copy, and paste are in the standard text editing sense.) 


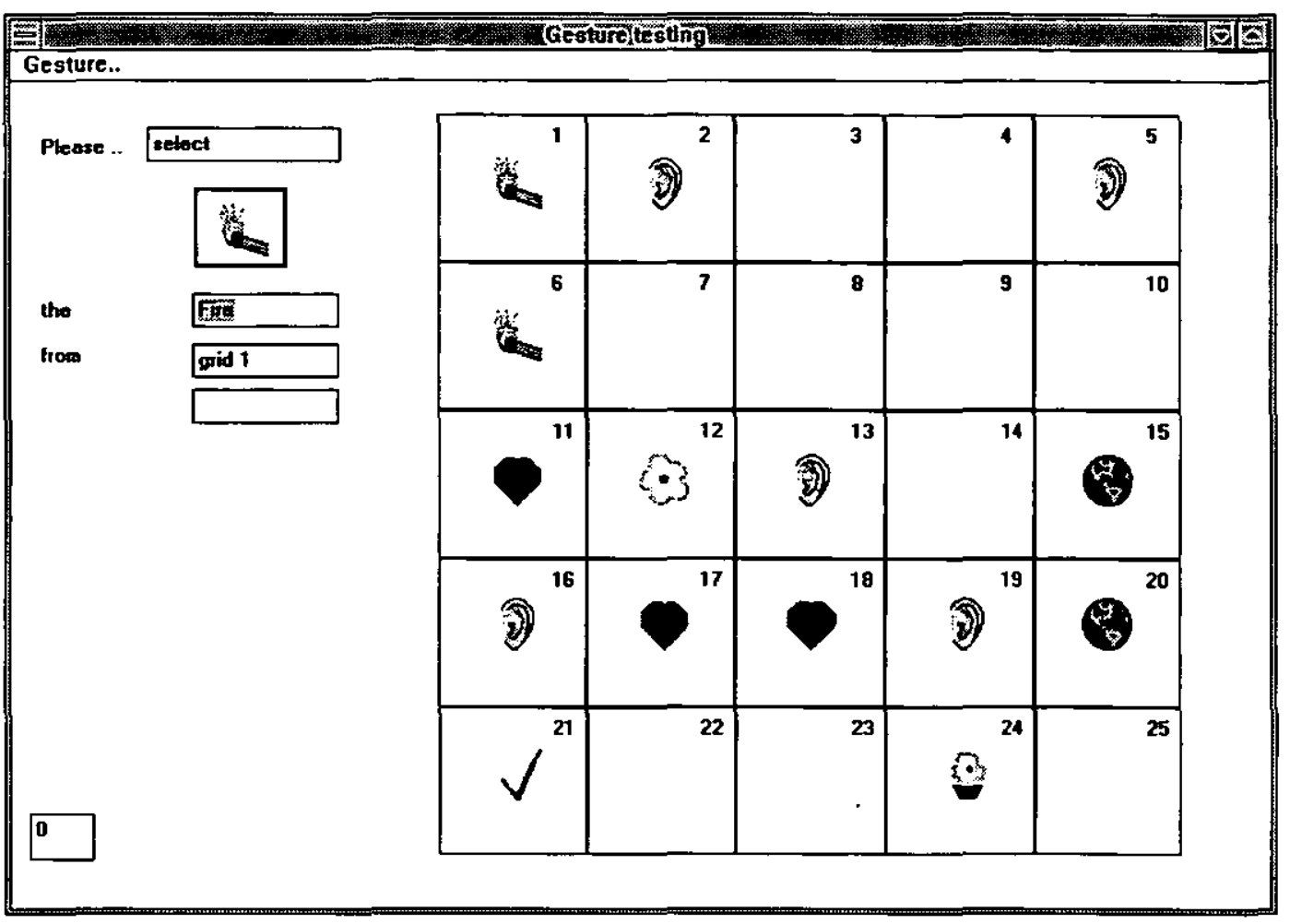

Figure 15: Shape manipulation application screen

In the gesture task, the user is presented with a square $5 \times 5$ grid (see Figure 15). Each grid location could contain one of seven different shapes: a heart, a flower pot, a flower, an ear, a lighted match, a world and a tick (either taken from the MSWindows clip art library or designed using MSWindows Paintbrush).

The user was asked to carry out a gesture by the automatic gesture request system (see Figure 16). All requests had the same basic format:

$$
\text { command-object-location(s) format }
$$

The number of locations was either one or two depending on the command. For example, the cut, copy and paste commands only requested a single location (in the case of cut and copy, the where-from location, and the case of paste, the where-to location). In contrast, the move command needed two locations: the where-from and where-to locations. 


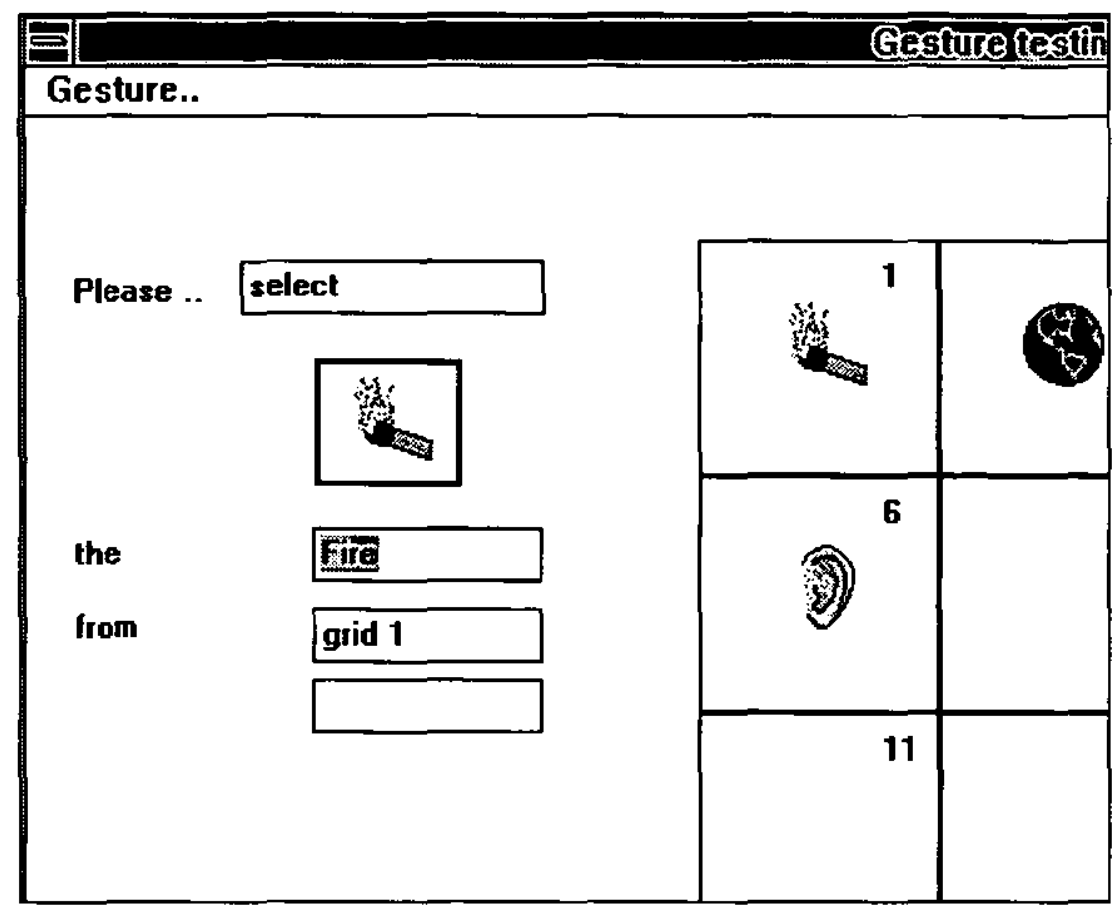

Figure 16: Screen shot of automatic gesture request area

Requests were of the form command-object-location(s), examples of which are shown below.

Cut the Tick from grid 12

Select the Ear from grid 19

Move the Heart from grid 1 to grid 5

Paste the Earth to grid 24

The gestures used to carry out these requests were a cross, circle, tick and lines of four different gradients, which were mapped to the four possible commands in the requests: cut, select (copy), paste, and move.

\begin{tabular}{lc}
\hline $\begin{array}{l}\text { Command } \\
\text { Request }\end{array}$ & Mapped Gesture \\
\hline Cut & $\boldsymbol{x}$ \\
Copy/Select & 0 \\
Paste & $\checkmark$ \\
Move & $/$ \\
\hline
\end{tabular}

Table 6: Mapping of command requests to gestures 
The automatic gesture request generator provided an on-screen prompt, requesting that a particular gesture be performed by the user. The system noted the expected gesture, and its recognition result and recognition parameters derived from the performed gesture. Based on this recognition result, the system performed the appropriate action, following the gesture made by the user (e.g. move the appropriate icon from one grid to another, if the move gesture had been recognised appropriately). In the cases where a cut gesture was requested, the system would wait for the two components of the gesture to be made by users, the first and second strokes of a cross. Gesture requests were generated randomly (using a pseudo-random number generator), and were therefore not in any particular order.

In the first experiment, no error correction capabilities were present. Therefore, errors arose when users did not follow the on-screen requests correctly. In most cases of error, users had misread the request, gesturing in the wrong grids, or became confused and performed a different gesture to the one requested. The second experiment therefore included error correction capabilities. The system rejected any attempted gesture which used incorrect grid numbers. This error correction more effectively 'forced' users to provide gestures as requested, and also limited error data. This feature was the only difference between the first and second experiments, and was not implemented in subsequent versions of the system. This feature was necessary at this stage to ensure that reasonable gesture attempts were recorded by the system, so that accurate gesture data was available for analysis (and subsequent classification).

\subsection{Experimentation}

A set of experiments was conducted, using twenty subjects in total. Ten subjects were used for the first experiment, and ten subjects for the second experiment. All subjects were computer literate but most were not familiar with using gesture as an input mode. In both experiments, users were asked to follow the on-screen requests, using the system described in the previous section and shown in Figure 15 and Figure 16. 
Before the experiments, users were given information on the format of the experiments and the actions they were required to perform.

The instructions and guidelines included the following:

- they were told the number of gestures that they would be prompted to make (128 gestures per experiment)

- they were informed that no time restrictions were in force

- they were asked to use the touchpen supplied (rather than the less precise use of the finger)

- they were instructed on the mapping of the possible system commands to the possible gestures

(see Appendix B for an example guide sheet).

Each user was asked to gesture across two sessions to minimise user fatigue, producing a set of 128 gestures per user. Thus in total, each experiment provided 1280 example gestures, stored in data files with a set of parameters for each gesture, the requested gesture, and the recognised gesture result. Each gesture produced a case, and each dataset from each user contained 128 cases.

As the first experiment did not have error correction capability, there were some cases in the datasets which were incorrect, as users had not performed the requested gesture. This made a classification of these gestures, based on a comparison between the requested and attempted gesture impossible. Therefore, these error cases were removed from the datasets of the first experiment, giving a total of 923 cases across the 10 datasets. The datasets from the second experiment were complete, giving a total of 1280 gesture cases.

\subsection{Classifying the gestures}

Most approaches to two- and three-dimensional gesture recognition use statistical methods to process the recognition data (Bordegoni \& Faconti, 1996). Newby (1993) used statistical similarity (the sum of squares approach), while Rubine 
(1991) used a linear classifier on a set of gesture features, which had been extracted from example gestures.

As the gesture recognition system was developed, and the requirements of a multimodal input system were considered, induction was an obvious classification model to use for our system. Inspection of the gesture data showed variability, as expected, between the subjects. A classification model had to take account of this variability, to produce general yet effective models.

The parametric approach of linear classifiers did not suit the nature of the gesture data, as the identification of critical attributes which recur in the induction rules, is crucial to the classification of gestures. The use of parametric models and linear machines is perhaps more suited to optimising gesture recognition as in the case of Rubine (1991). Also, the flexibility which rule induction provides, through the partial match possibilities which induction rules can allow, was an ideal approach for a gesture recognition system which was to be integrated into a final multimodal system.

Rule induction as a form of classification for two-dimensional gestures does not appear to have been used elsewhere. However, a related use is that of twodimensional object recognition in X-ray images (Maloof \& Michalski, 1995; Maloof \& Michalski, 1997). In these studies, various forms of inductive techniques for learning about two-dimensional shapes in X-ray images have been compared. Similarities to the work described here, include the decomposition of the focus of analysis (gestures in our case, objects on X-rays in their case) into sets of descriptive parameters and attributes. These sets are then provided as training examples to the induction programs, which produce general descriptions and rules, which can then be used to classify previously unseen objects or gestures.

The benefit of the classification process is described as the 'flexible matching method' (Maloof \& Michalski, 1997) which allows a 'degree of match' to be obtained in the recognition process, rather than the more inflexible approach of a 
strict yes-or-no match. It is this concept, the flexibility of degree of match which we concentrate on in the following discussion. This is a highly appropriate method for the classification of two-dimensional gestures, which have been described by a set of attributes, some of which may apply to the gesture and some of which may not. The approach also provides us with a set of parameters to be used in the multimodal system.

\subsubsection{Brief description of Rule Induction}

The use of rule induction is suited to particular classification tasks which have the following properties: (Quinlan, 1993).

\subsubsection{Attribute-value description}

The data used for analysis should be expressible as a set of fixed attributes or properties. The properties should not differ from case to case (a case, in the work described here, is the set of calculated parameters from one individual gesture).

\subsubsection{Predefined classes}

The classes into which cases can be grouped must have already been previously established. This is called supervised learning in the field of machine learning (our classes are the set of possible gestures).

\subsubsection{Discrete classes}

Classes must be distinct from one another i.e. mutually exclusive. A member of one class cannot be a member of another. There must also be considerably more cases than classes. This property also holds for the gestures, as one gesture cannot be classified as another gesture simultaneously. Additionally, there will be substantially more gesture cases than classes, as the experiments request more than 100 gesture examples per subject (over a set of eight gesture classes). 


\subsubsection{Sufficient data}

As induction looks for patterns in data, it follows that there must be enough cases for robust patterns to be identified. The number of attributes and classes, as well as the complexity of a classification model, all affect the amount of data required. Again the gesture data satisfies this condition, as each experiment provided around 1000 cases in total.

\subsubsection{Logical classification models}

The output from the induction software used here, produces a logical rather than an arithmetic model of classification. Arithmetic models are those such as linear classifiers or machines which calculate a class based on weights of attributes. These models assume that the concept of the class can be expressed as a linear combination of the attributes in some way. The class is then decided by the optimum combination of attributes which is the best fit. The logical models give more flexibility than the arithmetic approach, providing more flexible matching criteria.

\subsubsection{Using rule induction in gesture recognition}

As a form of classification, induction is suited to attributes which can be expressed either discretely or numerically. For instance, in our gesture data, there are some tests which return Boolean values of true or false. The gesture can then be described by these Boolean results, and are clearly not numeric. Standard statistical methods do not allow for such descriptors. Also many are parametric, i.e. a model is assumed and then appropriate values are sought for the model's parameters from the data. An example of this is a linear classifier, where the model is the class which can be expressed as a linear combination of the attribute values in some way (the arithmetic model as described above). Then, when training cases are supplied, the optimum linear combination of attributes that best fits is searched, so that the class can be decided (Quinlan, 1993). 
The machine learning program, with the programs C4.5 and C4.5rules (Quinlan, 1993) was chosen. The C4.5 program generated decision trees based on data supplied to it. C4.5rules generates production rules, by modifying the decision trees generated from the $\mathrm{C} 4.5$ program. This modification process firstly rewrites the decision trees as rules (where every path from the root of a tree to a leaf gives one initial rule (Quinlan, 1993)). The rules are then simplified, removing irrelevant conditions that did not discriminate between classes. Finally, the rules are ordered to minimize the possibility of false positive errors.

Quinlan noted that

'This process leads to a production rule classifier that is usually about as accurate as a pruned tree, but more easily understood by people.' (Quinlan, 1993).

Similar but not exactly equal performance rates were therefore expected from the decision trees and production rules. C4.5 and C4.5rules evolved from Quinlan's earlier work on ID3, which had more limited capabilities. For instance, early versions of ID3 did not accept numeric parameters in the classification models (Quinlan, 1993). This was not suitable for the gesture recognition system which produced both discrete and real-valued attributes. C4.5 and C4.5rules permitted both numeric and descriptive parameters, and also generated both decision trees and production rules.

\subsubsection{Preliminary results}

The data gained from these initial gesture-only experiments was first analysed using the C4.5 and C4.5rules machine learning software (Quinlan, 1993). The parameters from each recognised gesture were processed into a comma-delimited text format for the software, together with the classification of what the gesture attempt was. This classification information was taken from the gesture request information, which had been stored with the recognised gesture parameters during the experiment. Therefore, in the terminology of the machine learning system, each gesture represented a case, the calculated parameters of the gesture 
represented the attributes of the case, and the requested gesture represented the class of the case.

The cases from each subject were used as sets of training examples for the machine learning system, to produce decision trees (using the C4.5 program). These decision trees were then processed to give production rules (using the C4.5rules program), which indicated the likely class that a gesture belonged to, based on only that single user's data. The generated production rules varied in the number of conditions they contained, and in the individual parameters included in the body of the rules.

The format of the rules is as follows:

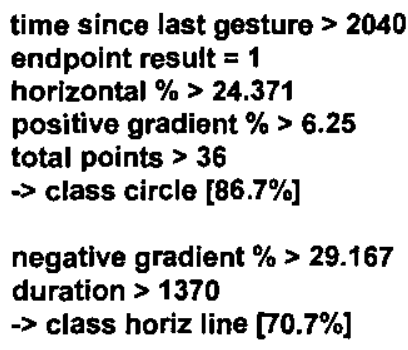

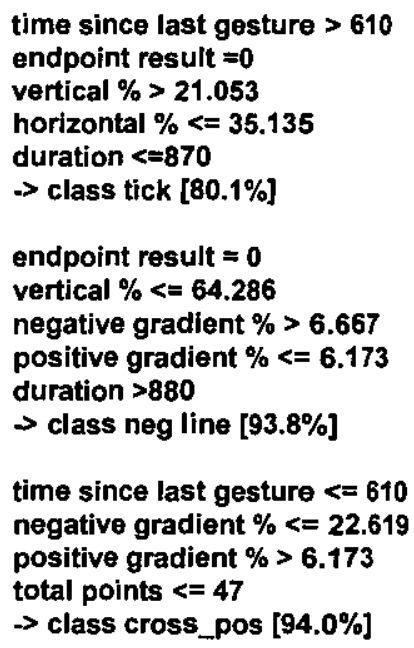

Figure 17: Example production rules

Production rules present a form of classification which is easier to read and understand, when compared to decision trees. Decision trees can have numerous levels, or nodes, which can be very difficult to follow, even for relatively simple classifications (Quinlan, 1993). Percentage confidence estimates for the efficiency of the trees and rules in classifying further, unseen data were also given by the C4.5rules software (as indicated in the example rules, class tick [80.1\%]). It was then possible to test the trees and production rules on the data from all the other users in the group, to gain an understanding of how efficient the models would be in classifying unseen gestures. 
The machine learning software (Quinlan, 1993) generated several structures and information parameters about the data:

- decision trees (using C4.5) and production rules (using C4.5rules)

- number of input parameters used to generate rules

- $\quad$ predicted error rate

- number of times the rule was used to classify cases

- number of cases which satisfied a rule but were of the wrong class

- confusion matrix produced to show where mis-classifications occurred

The decision tree analysis produced overall predicted error rates using the training data (the data from each subject), and also gave actual error rates using the test data (the data from every other subject). The production rule analysis of the data gave differing rules and results, with different parameters and ranges.

The C4.5rules software also generated analysis information about the supplied data. Evaluation tables and confusion matrices, showing the classifications on the training and test data, detailed the rules used to classify the cases and where misclassifications had occurred. The actual error rates were also given for the classifications presented. Examples of the matrices and evaluation tables are shown in Table 7 and Table 8. The example is a classification from one subject in the second experiment, using the production rules from that user, on the unseen cases from the nine other subjects in the experiment (test data).

The set of classes was defined to be the set of all gestures in the system. Several factors affected the rules and trees produced: the number of classes, and the inclusion or exclusion of a 'cross' class in the data. For instance, the cross contained two component gestures, a line of negative gradient and a line of positive gradient, in some order, which intersect one another. However, these would differ from the move gestures of negative and positive gradient, since crosses are only performed within a grid, rather than across grids in the manner of move gestures. Therefore two new classes were added, that of cross_neg and cross_pos and the total set of classes was defined to be as follows: \{circle, tick, vert line, horiz line, neg line, pos line, cross_pos, cross_neg\}. 


\begin{tabular}{lllllll}
\hline Rule number & Size & Error & Used & Wrong & Advantage & class \\
\hline 17 & 4 & $11.8 \%$ & 160 & $46-28.7 \%$ & 22 & circle \\
14 & 5 & $9.4 \%$ & 68 & $17-25.0 \%$ & 49 & tick \\
5 & 5 & $15.7 \%$ & 78 & $19-24.4 \%$ & 59 & horiz line \\
6 & 4 & $17.8 \%$ & 143 & $59-41.3 \%$ & 41 & neg line \\
7 & 2 & $29.3 \%$ & 98 & $64-65.3 \%$ & 29 & neg line \\
11 & 3 & $24.3 \%$ & 192 & $83-43.2 \%$ & 81 & pos line \\
8 & 3 & $50.0 \%$ & 36 & $26-72.2 \%$ & 6 & pos line \\
12 & 5 & $12.9 \%$ & 97 & $34-35.1 \%$ & 4 & vert line \\
10 & 2 & $48.5 \%$ & 75 & $28-37.3 \%$ & 45 & vert line \\
3 & 2 & $21.6 \%$ & 80 & $12-15.0 \%$ & 67 & cross_pos \\
1 & 2 & $22.9 \%$ & 95 & $30-31.6 \%$ & 56 & cross_neg \\
\hline
\end{tabular}

Table 7: Example of an evaluation table

The table shows the rule number used to classify the cases (Rule number), the number of parameters in the rule (Size), the estimated error of the rule in classifying other unseen cases (Error), the number of times the rule was used in classifying cases (Used), the number of cases incorrectly classified using the rule (Wrong), the net benefit of keeping the rule (Advantage) and finally, the Class which the rule applies to.

Confusion matrices provide more detail of where mis-classifications occur when using the production rules. The following confusion matrix is from a subject in the second experiment, whose production rules were used to classify the unseen data from the other nine subjects in the experiment.

\begin{tabular}{|c|c|c|c|c|c|c|c|c|c|}
\hline (a) & (b) & (c) & (d) & (e) & (f) & (g) & (h) & $\begin{array}{l}\text { Total } \\
\text { cases } \\
\text { per } \\
\text { class }\end{array}$ & \\
\hline 122 & 1 & 7 & & 1 & 3 & 1 & 9 & 144 & (a) circle \\
\hline 28 & 51 & 26 & & 2 & 23 & 7 & 7 & 144 & (b) tick \\
\hline 3 & 4 & 110 & & 7 & 18 & & 2 & 144 & (c) vert line \\
\hline 3 & & 6 & 59 & 56 & 18 & & 2 & 144 & (d) horiz line \\
\hline 6 & 1 & 7 & 8 & 118 & 4 & & & 144 & (e) neg line \\
\hline 3 & 10 & 1 & 8 & 3 & 119 & & & 144 & (f) pos line \\
\hline 16 & & 5 & & 2 & 42 & 68 & 10 & 143 & (g) cross_pos \\
\hline 9 & 1 & 10 & 3 & 52 & 1 & 4 & 65 & 145 & (h) cross neg \\
\hline 190 & 68 & 172 & 78 & 241 & 228 & 80 & 95 & 1152 & $\begin{array}{l}\text { Total cases } \\
\text { identified per } \\
\text { class }\end{array}$ \\
\hline
\end{tabular}

\section{Table 8: Example confusion matrix}

The matrix shown in Table 8 displays the classification details of all cases in the test data. Each row represents the total number of cases which were actually of 
that class, while each column represents the total number of cases identified as that class. Therefore, the diagonal through the matrix represents the correct classifications of each gesture.

In the first row, 122 circle cases were correctly classified as circles, 1 was classified as a tick, 7 as vertical lines, 1 as a negative line, etc. This provides a guide to the actual performance of the production rules in classifying the cases, which is of assistance later when the production rules are used in the final gesture recognition system. Inspection of the evaluation tables and classification matrices allowed selection of the rules which gave the best performance in classifying unseen data. This helps to produce a final gesture recognition system which is equipped to make 'best guesses' about gestures made.

\subsection{Analysis of gesture data}

A complete data set was generated from the first group of ten users, and the second group of ten users, to give overall decision trees and induced rules for each experiment. The predicted error rates could not give an insight into the possible performance of these rules in recognising gestures, unless tested on previously unseen cases. Therefore, to gain figures on how accurate and useful each set of trees and rules (from each individual user) could be, each tree and ruleset was tested on a dataset of unseen cases: the data from all the other users in the group.

The predicted error rates were produced by the software based only on the training data supplied at the time of compilation. These represent the prediction of the percentage of unseen cases which the system would expect to classify correctly. Additionally, the predicted error rates are based on the success of one user's rules classifying their own gestures, which results in too low a prediction of variability. These figures are more an opinion of the system than a tangible guide. This is evidenced in the fact that the predicted error rates are fairly low, with overall classification confidences all greater than $78 \%$. Clearly, only unseen 
cases analysed under the produced trees and rules give a guide to their actual efficiency. The data from other users also provides a greater variability and a realistic test set.

\subsubsection{First Experiment}

\subsubsection{Error rates}

The decision trees and production rules from each subject were then used to classify the data from all other subjects in the group (nine other datasets). The induction software (Quinlan, 1993) had the capability to use the generated trees and rules to classify further unseen data. This classification gave further evaluation tables, confusion matrices and error rates from the trees and rules. Three main error rates were generated: predicted decision tree error rates, actual decision tree error rates, and actual production rule error rates.

The predicted and actual decision tree error rates represented the performance of the whole tree on the provided test data (the other nine datasets). The actual production rule error rate represented the overall performance of all the rules on the test data. There was no overall predicted error rate for the production rules, as predictions were provided for each individual rule.

Figure 18 shows a comparison of the three main error rates for the first experiment, where the decision trees and production rules per subject were tested on the data from all other subjects in the group (recall that different error rates are generated by the decision trees and production rules, as the production rules represent a modified form of the decision tree, not a replica of the decision tree, in rule format). 
FIrst Dataset

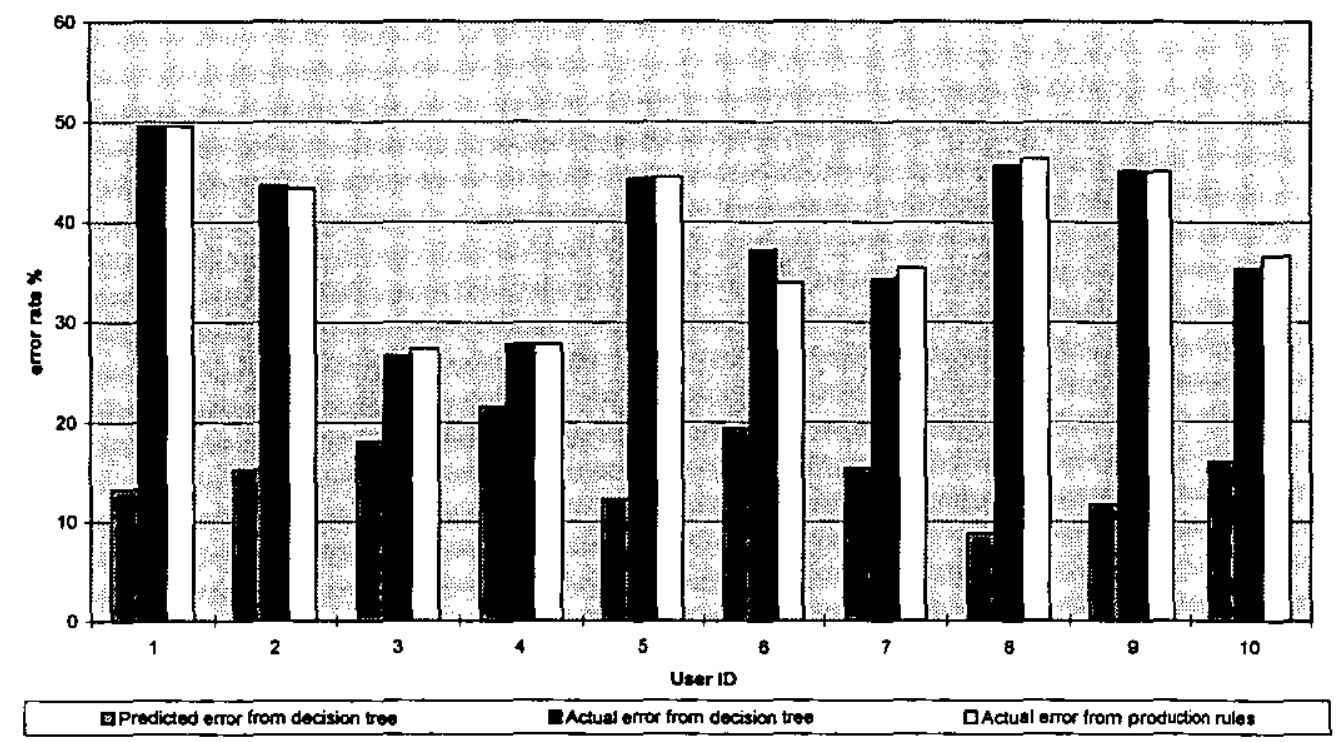

Figure 18: Comparison of predicted \& actual decision tree error rates, and actual production rule error rates, Experiment 1.

The test data which each tree and ruleset was applied to, was between 800 and 923 cases in size. The use of test data gave more realistic error rates nearer to expectations, than the predicted error rates. The results of some subjects showed that when their individual classification trees and rules were applied to the test data, these models performed the classification task poorly. For instance, the rules of User 8 (Figure 18), who had the lowest predicted decision tree error rate of $8.8 \%$, when applied to the test data, gave high error rates of $45.6 \%$ and $46.3 \%$ (actual tree and actual rule error rates, Appendix B, Table 24). This could be. attributed to the individuality of each subject, in the way they performed gestures, and the classification models which they generated.

The average error rates over all users, for each error rate type, were $15.13 \%$, $38.95 \%$, and $38.96 \%$ (predicted decision tree, actual decision tree, and actual production rule respectively). This shows that the actual error rates for both decision trees and production rules are almost equal for most users, as was expected from Quinlan's comparison of the two approaches. 
An overall predicted error rate for the production rules was not generated by the system. Instead, predicted error rates were given for each individual rule. In all cases the actual error rate was either higher or significantly higher than the predicted error rate, when unseen cases were tested using the rules. Analysis and comparison of individual rules was difficult. In some cases rules were not produced for a particular gesture in the first dataset. This was mostly due to the observed errors found in the data, when users had not performed the requested gesture and action.

The standard pattern of behaviour for most users was to be cautious in making gestures, both in the time they took in performing gestures, and the time they took between gestures. Most users exhibited an overall hesitancy in performing gestures, and this greatly affected the success of the cut actions, which were dependent on there being a reasonably small time gap between the strokes of a possible cross (this gap was set to 0.8 seconds for the gesture only experimentation.) Obviously, different results would have been achieved had this gap been set higher. Setting the time-gap to a higher level however could have proved counter-productive, since if it was too high it may have resulted in unconnected gestures being considered related by the gesture recognition system (the problem of segmentation, see Section 4.3.4). Many pairs of gestures would have been interpreted as possible cut actions. Therefore the timegap cut-off level was kept at the 0.8 secs level.

\subsubsection{Interpretation}

Comparison of the individual production rules was difficult. In some cases there were no rules found to classify a particular gesture. This could be attributed to the imbalance of gesture types in the first dataset. For instance, the first experiment generated random move requests which led to a random rather than controlled number of associated line gestures. Thus, in one dataset, no positive gradient line gestures were requested of the user and so, obviously, no patterns for this class of gesture were found. With these types of problems, the classification models gained from the first were inefficient and incomplete. 
These first experimental results were used to generate an improvement plan for the next set of experiments. Firstly, the improved system needed to take account of observed errors made by users, and to request that they repeat the gestures in such cases. For instance, errors generated by users performing gestures in the wrong grids to those requested, could result in the wrong gesture being made (e.g. a line with a gradient different from the request).

Also, the data needed to be balanced over all gestures to ensure relevant rules could be deduced about each gesture. Previously, the production of data was governed by the number of actions (cut, copy, paste, move) that had been performed instead of gestures ( 1 cut gesture [ 2 component gestures], 1 copy gesture, 1 paste gesture, and 4 move gestures). Finally, another set of gesture experiments would provide another dataset to generate decision trees and rules, and would also provide another test dataset.

\subsubsection{Second Experiment}

The second experiment used ten subjects (different subjects from the first experiment), each performing 128 gestures around the same numbered grid (recall Figure 15, page 97). The error correction facility automatically requested that subjects repeat a gesture if it was performed in the wrong grid. Therefore, there were no errors present in the complete dataset containing 1280 gesture samples. As in the first experiment, these gesture cases were processed by the machine learning system, using C4.5 and C4.5rules (Quinlan, 1993), per subject and over the total dataset, to provide decision trees and production rules.

\subsubsection{Error rates}

The generated decision trees and production rules from each subject were used to classify the unseen data from all other subjects in the second experiment (nine other datasets). As seen previously in the first experiment, this classification produced three types of error rates, to provide guidance on the performance of the trees and rules on classifying other unseen data. The predicted decision tree error 
rates, actual decision tree error rates and actual production rule error rates are plotted in Figure 19 below.

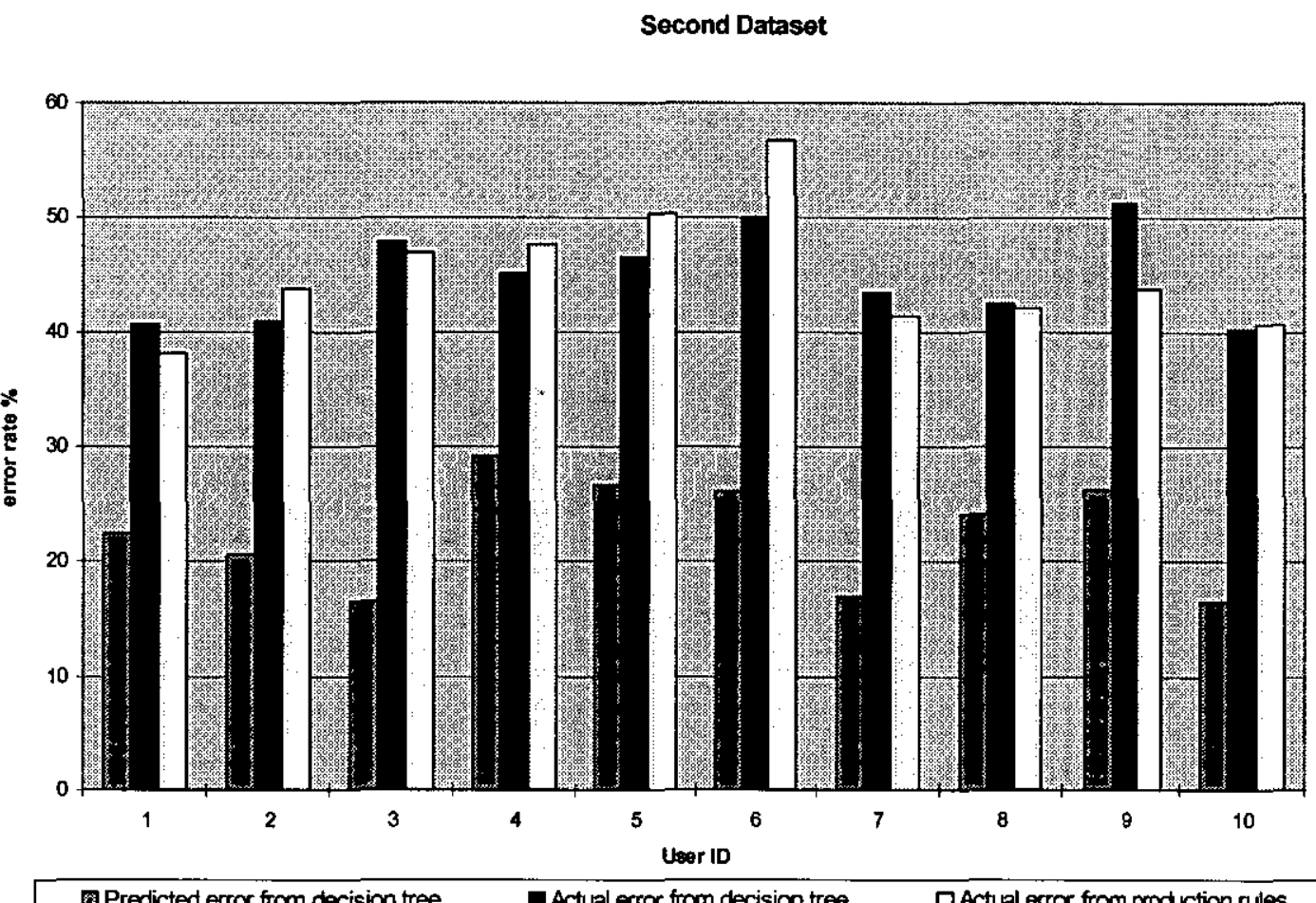

Figure 19: Comparison of predicted $\&$ actual decision tree error rates, and actual production rule error rates, Experiment 2.

The graph shows that the actual error rates were again always higher than the predicted error rates. The graph also shows that the comparison of decision tree error rates and production rule error rates varied, as expected. Some subjects gave tree rates higher than rule error rates, while other subjects demonstrated the opposite effect.

The error rates of the decision trees and production rules generated from the second dataset, were higher on average than those generated from the first dataset. A plot of the averages for the second dataset are shown beside the averages from the first dataset in Figure 20. 
Averages from First and Second Datasets

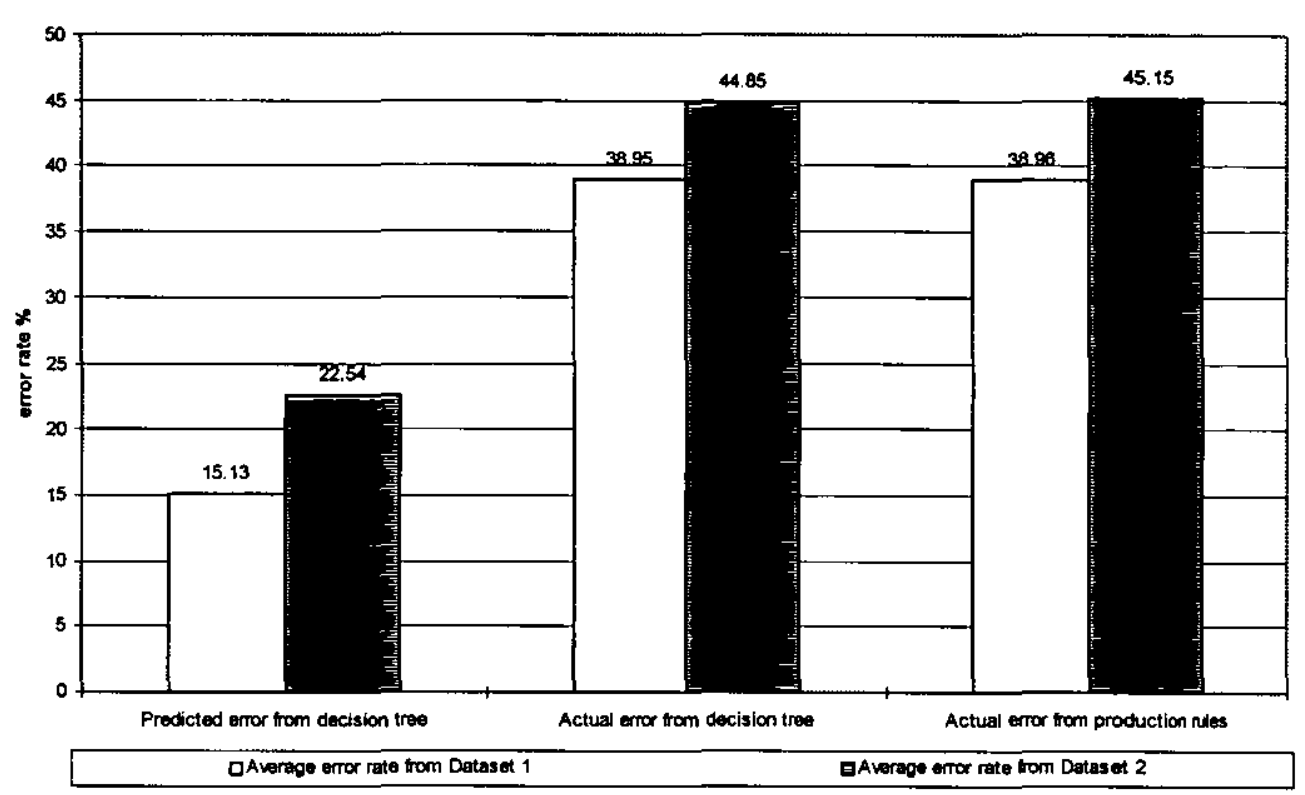

Figure 20: Average error rates per dataset, for predicted decision tree, actual decision tree, actual production rule

The averages shown in the first column of each group represent the average error rates of the decision trees and production rules, generated from the first dataset. Similarly, the second column of each group represents the average error rates generated from the second dataset. Therefore, the observation that the second dataset generated trees and rules with higher error rates than the first dataset is confirmed.

This can be attributed to two main factors: the error rates apply only to the training data which generated the models initially, and therefore the error rates represent only how successful one individual's trees and rules were at classifying their own data. This is not a particularly objective measure of the success of the model. Secondly, the lower number of cases in the first dataset (923 cases versus 1280 cases in the second set) provided less training cases per subject, and it could be argued that the generated models were less realistic and efficient than those from the second set, which had a higher number of training cases (recall that the 
number of training cases provided would affect the classification model produced, Section 5.5.1 and Quinlan, 1993).

As expected, the decision trees and production rules generated from both datasets show that both methods had similar performance in classifying the data. The error rates of around $40 \%$ are higher than desired for usable sets of trees and rules. However, a better test of the generated models would be their performance on classifying unseen gesture data. Therefore, the decision trees and production rules generated from the first dataset were used to classify the data from the second dataset (Case 1, Figure 21).

Similarly, the decision trees and production rules generated from the second dataset were used to classify the data from the first dataset (Case 2, Figure 21). Recall that the first dataset was smaller than the second, due to the removal of the errors, giving dataset 1 a total of 923 cases, while dataset 2 contained 1280 cases. Thus, column 1 results are made with the number of test cases greater than the number of training cases, while column 2 results are the opposite, the number of training cases exceed the number of test cases. 


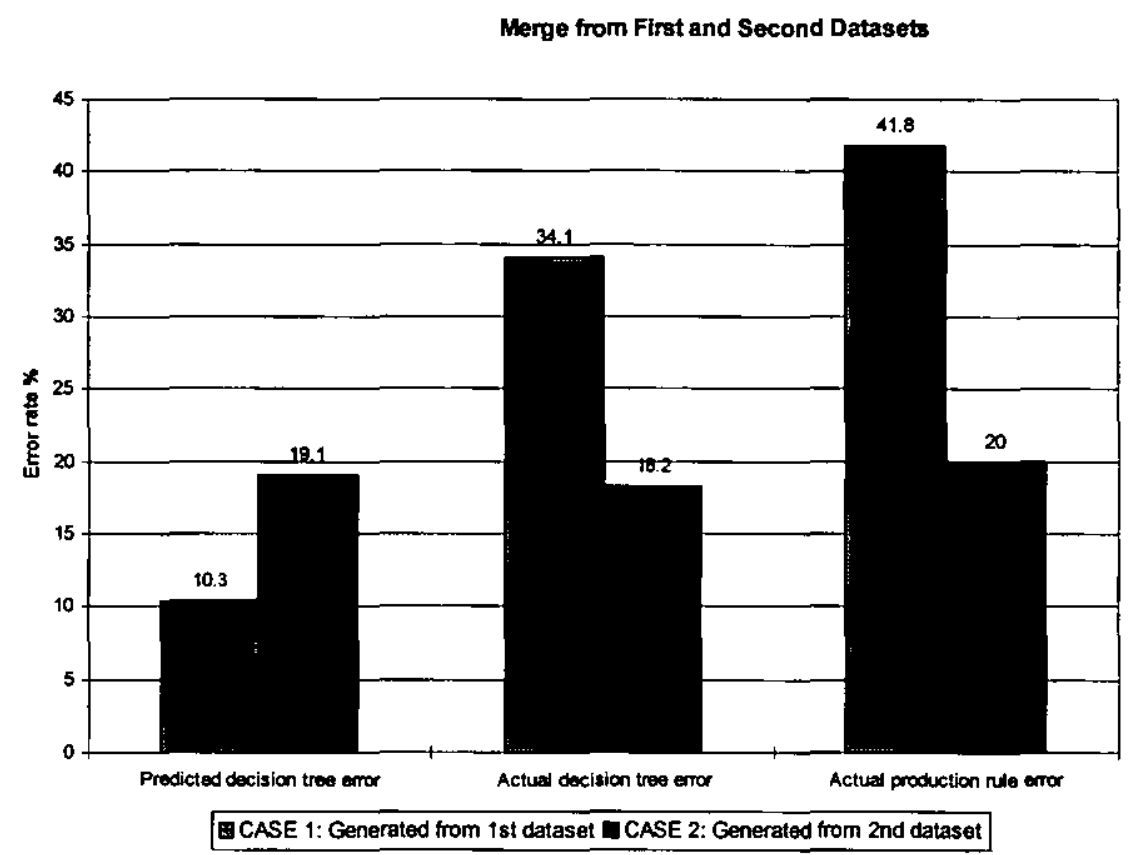

Figure 21: Average error rates (predicted decision tree, actual decision tree, actual production rule)

The chart shows that for the actual error rates, a lower error rate came from both the decision trees and production rules generated from the second dataset. The trees and rules generated from the first dataset gave higher actual error rates, although the predicted decision tree error rate was lower than that rate from the second dataset. This difference in performance and error rates can be attributed to the difference in the number of training cases which were involved in the generation of the classification models (decision trees and production rules). The number of test cases in comparison to training cases may also have had a bearing on the results.

The number of training cases was greater for the second set of trees and rules, which is more likely to have generated a more efficient set of models than from the first set. The number of training cases serves as evidence upon which the decision trees and rules are built. Therefore, the higher number of cases, the greater the amount of evidence to sort through to find classification patterns (Quinlan, 1993). Additionally, as the production rules and decision trees were 
generated from the data of ten subjects, this provided a more balanced model which was not dependent on the gesture patterns of one individual. This would eradicate possible peculiarities and characteristics associated with only one subject, and give a more general example gesture base, which could then generate a wider scope of classification models.

Further analysis showed an increase in error rates, when subsets of the training data were used to induce new trees and rules. This confirmed the explanation of why the second dataset gave lower error rates, since this reduced the number of training cases. However, some unexpected results were revealed. When the set of training cases for case 1 was reduced by one user, i.e. by 128 cases, the error rate fell. Similarly when another user's data was removed, again the error rate fell. Only when another user's data was removed and the number of training cases fell below the number of test cases did the error rate rise again. This could be attributed to inefficient nodes and rules being removed in the smaller training set examples (Quinlan, 1993).

Using the merged data gave lower error rates than any of the individual or average error rates, making the merged trees and rules the preferred choice when choosing the production rules for the final gesture recognition system. The decision tree produced lower error rates than the production rules. However, as the size of the decision tree and included sub trees amounted to twelve A4 pages, the production rules were chosen instead. The generation of a robust gesture recognition system was the main aim of these gesture-only experiments, rather than investigation of an optimal method of recognition. Therefore, the difference in error rates between the methods (1.8\%) was not of significant concern.

\subsection{Final gesture recognition implementation}

\subsubsection{Refining the gesture system}

Gestures were classified in the gesture recognition system, used in the experiments (Section 5.4), by a series of heuristic tests (Section 4.4.1). However, 
some of the calculated parameters were not included in tests for the identification of some gestures. While these tests made use of some of the calculated parameters for each gesture, observation alone was not enough to determine expected values for some parameters of particular gestures. For instance, the test determining the distance between the start and endpoints of gestures, the endpoint test, was introduced primarily for the basic identification of circles. However, this test may have relevance for other gestures, such as tick and line gestures. Therefore, the production rules highlighted parameters which may have been previously omitted in the classification of some gestures.

The set of production rules generated from the second experiment, was used to refine the gesture classification part of the system. On average between 5 and 8 rules were used per gesture. Any generated rules which had particularly high error rates were excluded from the set of rules. Subsets of the production rules were also used in the final system. Where a rule had, for example, six parameters, then several sub-rules could be included with between two and five parameters, with an appropriate reduction in the estimated success of the rule in classifying gestures. Sub-rules with only one parameter, were excluded from the set, as these would have limited use, and probably apply to all gestures.

These production rules were used in the knowledge bases of a truth maintained blackboard system.

\subsubsection{Truth maintenance and blackboard system}

The use of the chosen production and heuristic rules gave a framework for determining recognition possibilities. Therefore, a reasoned method of generating a set of possibilities for each gesture was required in the final gesture system. It was important to generate a number of possibilities, in contrast to the earlier systems, where only one choice could be made.

It was a requirement that a list of possibilities was produced, ranked into some order. A truth maintenance system could aid this process, since an assumption- 
based truth maintenance system operates by being able to maintain several, possibly inconsistent views of a problem. In our case, the inconsistent views would be the set of gesture possibilities, which would present an inconsistent view of one gesture, but provide suggestions for its possible recognition. The blackboard system would handle this problem, aiding in the efficiency of the problem solving.

Initially, when truth maintenance and blackboard systems were investigated for this research, the possibility of using these systems for the processing of the information from multiple input modes was considered. However, this plan was eventually discarded, as there were two main factors which made implementation of the integration of modes problematic within the truth maintenance system:

- handling of time-stamp information, crucial in the integration of modes, was difficult within the Prolog (Bratko, 1986) framework of the truth maintained blackboard system

- validation of the multimodal possibilities, using current system task and context knowledge (based in the $\mathrm{C}$ framework of the application) would have been difficult to implement in real-time

However, a truth maintained blackboard system was still appropriate for handling the gesture aspects in the final multimodal system.

\subsubsection{Properties of Truth Maintenance Systems}

An expert system is an expert problem solver, which has access to a knowledge base, and uses reasoning methods to attempt to solve the problem posed. The knowledge base is often in the form of rules, which can be consulted by the reasoning mechanism during problem solving (Rich \& Knight, 1991). A blackboard system is a system of managing the work done towards the solution of a particular problem. The analogy which provides the name, is that of a collection of experts around a blackboard, each with their own specialised knowledge of one area. Their only means of communication is a blackboard, upon which they write and update their current knowledge and interpretation of the problem. A blackboard system is involved more in the management of solving a problem, than in the actual solution itself. 
A Truth Maintenance System is a way of supporting possibly incomplete information and assertions, and of therefore supporting nonmonotonic reasoning (a method of reasoning which can work with incomplete, inconsistent information, and can make inferences based on a lack of knowledge, rather than the existence of knowledge) (Rich \& Knight, 1991).

Some truth maintenance systems also perform dependency-directed backtracking, which is a concept used to process inconsistencies more effectively. If a conventional blackboard system encounters inconsistencies, it performs some type of backtracking to distance itself from the inconsistency. However, a significant amount of work may be lost in this backtracking. If the backtracking is directed at the source of the inconsistency, this may improve the efficiency of the system (Hinde et al, 1989).

Standard truth maintenance systems (such as justification and logic-based systems) use this method of backtracking when inconsistencies are met, as only one line of reasoning is supported at a time (Rich \& Knight, 1991). Assumptionbased truth maintenance systems allow multiple paths of reasoning to exist in parallel, and therefore can maintain inconsistencies which the other types of system strive to avoid. Assumption-based truth maintenance systems do not therefore perform dependency-directed backtracking (Rich \& Knight, 1991). An assumption-based truth maintenance system is therefore the type of system used in the proposed gesture analysis system.

\subsubsection{Relevance to gesture recognition}

The parameters calculated for each gesture (Section 4.4.1) were general and could sometimes give an incomplete picture of a gesture. If a particular test using the parameters returned a negative rather than positive result, this could represent a gap in the knowledge about that gesture. The false result may not necessarily prove anything about that gesture, only that a particular property did not hold true at a certain level. This made it more difficult to make conclusions about gestures in the overall heuristic rules applied to the gestures. The alternative approach of 
the production rules which included more information about the parameters and specific levels which they should hold to, gave a level of information ideal for use in a truth maintenance system.

The ranked-bid truth maintained blackboard system used in the final gesture recognition system, was developed by Hinde et al (1989). Any facts or assumptions given to the blackboard system could be rated according their importance, so that when the system provided possible conclusions or solutions, it presented the most obvious conclusions before those of lesser weight or importance (Hinde et al, 1989). Therefore, in the context of our gesture recognition problem, the system could provide a list of possible gesture conclusions with associated ratings, based on the supply of a set of calculated parameters about that gesture. The parameters could be sent to the system in turn, the rules consulted, and the conclusions returned, to provide a consistent way of analysing and managing the data from the gestures.

\subsubsection{System implementation}

The system provided by Hinde et al (1989), was implemented in Prolog (Bratko, 1986), running on a SUN workstation. The gesture recognition system was implemented in the $\mathrm{C}$ programming language, running on a $486 \mathrm{PC}$. Therefore, an initial investigation of how to connect or combine the systems was required.

The most obvious initial solution was to have the capability of running the truth maintained blackboard system from the PC, in order to allow the system to be consulted from the $\mathrm{C}$ framework of the gesture recognition system, and from the same platform. One possibility was the use of the BinProlog environment, which could be installed on a PC platform. However, the conversion of the considerable system developed by Hinde et al (1989) from Sicstus Prolog on a UNIX machine (SUN workstation), to BinProlog proved to be both costly in time, and difficult to achieve without affecting the performance and integrity of the system. Therefore, another solution was required which would preserve the system in its original environment. 
TCP/IP sockets were therefore used to maintain a connection between the two systems via socket wrapper code (provided by Perkins, 1997) which passively redirected the socket input and output to the standard input and output on the UNIX machine (Figure 22). Sockets represent a communication interface between processes, whether these processes are local on the same computer or connected on remote computers. The socket interface was introduced initially for use on UNIX machines, and was then developed for MSWindows applications, usually known as Winsock (Sinha, 1996). This was the interface used for our system.

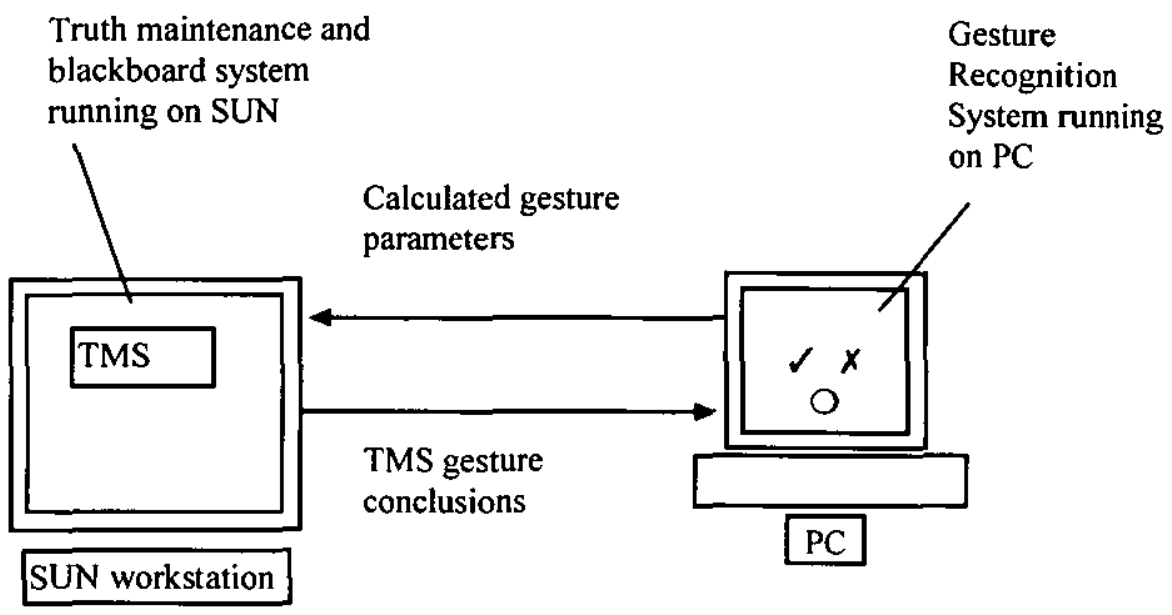

\section{Figure 22: Operation of truth maintained blackboard system between two platforms}

The truth maintained blackboard system was invoked each time a connection was made to the socket on the UNIX machine, from the gesture recognition system. The TELNET protocol was used to connect into the system from the gesture recognition system, running on the PC. The gesture recognition system accepted the raw gesture input (co-ordinates traced on the touchscreen), calculated a set of parameters, and then sent these parameters for the current gesture to the truth maintained blackboard system. The system on the SUN then relayed the blackboard conclusions back to the gesture system.

The gesture system then performed a considerable amount of parsing, to remove the blackboard syntax and extract the gesture conclusion from the blackboard 
messages. Parameters had to be sent in strict time order and format from the gesture system. The sending of any input from the PC was also dependent upon the successful response from the blackboard system, as a send could not occur if a receive was in progress. Similarly, the response from the gesture system to initiate and terminate the conversation was dependent on the response from the blackboard system.

Figure 23 shows the order of send and receives between the truth maintenance and blackboard system on the SUN workstation, and the gesture recognition system on the PC. This process would continue until all the gesture parameters for a single gesture had been sent to the truth maintenance system, and all output had been relayed back from this system to the PC.

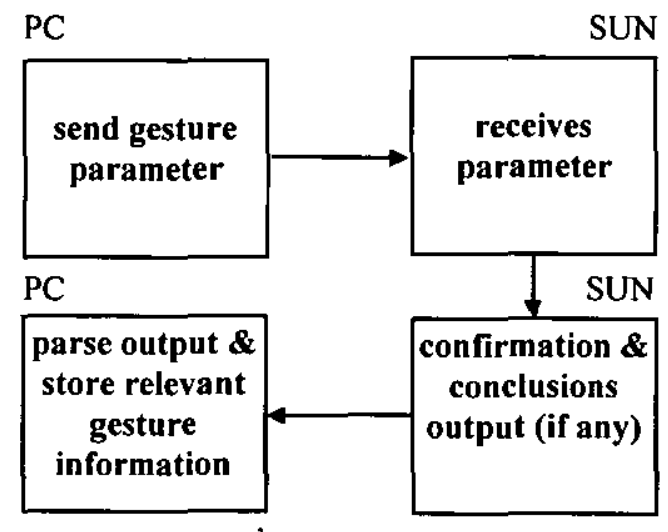

\section{Figure 23: Conversation between SUN workstation and PC}

Each time a gesture was made, the parameters would be calculated and sent to the truth maintenance system in this way. This process was therefore invoked each time a gesture was performed on the PC.

\subsection{Summary}

This chapter has described the experiments conducted using the two-dimensional gesture recognition system. The experiments provided data upon which induction could be performed. 
The induction generated a set of production rules which were used in the knowledge bases of a distributed truth maintained blackboard system, running on a SUN workstation, separate from the PC platform which accommodated the gesture recognition system. This was necessary due to the constraints of the PC which could not run the blackboard system efficiently. This actually proved to be efficient in terms of the real-time operation of the truth maintenance system, which could process the gesture data and return conclusions in real-time during the operation of the final multimodal system. Therefore, a distributed truth maintained blackboard system has been implemented. 
Section 3

\section{Multimodal Work}




\section{Chapter 6}

\section{The Design of the Multimodal Input System}

\subsection{Introduction}

This chapter examines the design decisions involved in the construction of the full experimental multimodal input system. The techniques used to integrate information from the component speech and gesture systems are outlined. The integration architecture of the multimodal interpreter is also described.

\subsection{Requirements of the Implemented System}

\subsubsection{Temporal Co-ordination}

In multimodal integration, the time-stamping of input information is important. This has been identified by Bellalem \& Romary, 1997; Koons et al, 1993; and Nigay \& Coutaz, 1993. Time-stamping is needed to map modes to tasks and to 
provide the system with the ability to determine relevant semantic, syntactic and context information. It assists the integrating system in determining when it is appropriate to fuse together the information from the separate modes. This property was called the fusion trigger by Faconti et al (1996), and referred to as the lexical aspect of fusion by Nigay and Coutaz (1993), who identified temporal fusion and data synchronisation as two important fusion properties.

Temporal fusion of information, from separate modes, can take place in several ways. The fusion may occur upon receipt of a particular type of input, which serves as a signal for the process of fusion to begin (for example, the fusing of available data after receipt of a gesture). Alternatively, fusion may occur only after a specific set of inputs has been processed (for example, fuse after a speech and gesture pair has been received). Fusion can also depend upon a specific temporal condition, where all the data received in a particular time frame is combined.

The best choice for temporal fusion is usually problem dependent, and the type of information being handled, its nature, and the outcome requirements all have a bearing on the decision. For instance, if incomplete data is a likely property of the information being handled, then fusion based on the condition of receipt of particular sets of information, may be appropriate. This ensures that the maximum possible amount of information takes part in the fusion process. In high volume input situations, fusion based on input received in a particular time interval may be the optimum choice ensuring that only the most relevant information (in the temporal context) is fused.

In our case, the decision as to when to fuse the data from independent speech and gesture input sources, into a single multimodal representation, is dependent upon how speech and gesture are handled in interaction with the system. The initial version of the multimodal system was a freely interactive version where speech and gesture could be used in any order. However, for experimental purposes, a constrained version was constructed in which the system defined the order of input. This system provided a far greater degree of experimental control. 


\subsubsection{Multimodal Interaction Style}

The main platform used for this project was a 486PC using Windows 3.1 (in the first two years of the project, Windows95 and high performance PCs were not available). Early on, performance tests indicated that whilst system execution was acceptable (in a real-time sense) for either gesture or speech input, processing of simultaneous input was not possible. Initially, it was thought that this might require a complete re-implementation on a Pentium PC. However, it was realised that simultaneous processing was not necessarily required for experimental purposes when investigating redundancy between modes. Furthermore, the sequential approach offered the advantage of more experimental control.

While sequential input may affect the natural style of interaction, it does not reduce the tangible benefits that arise from using more than one mode. In most systems the benefits occur because of the presence of additional information that is used in the multimodal integration process, so the temporal occurrence of the input is irrelevant in the context of multimodal recognition rates. It is accepted that sequential input will affect the users overall view of the usability of the system (there is little doubt that users will prefer simultaneous input because it is more natural (Oviatt et al, 1997)). However, our emphasis is on recognition rather than usability.

Thus, in the system a command involves either a speech act followed by a gesture act or vice-versa. For example, the command "Move the Earth from 12 to 16 " would involve a request to the user to speak this command, followed by a request to the user to execute a gesture to carry out the same command. Alternatively, the user could be asked to carry out the procedure in reverse, although this format was not actually used in experimentation.

The system also used constrained speech. Natural, spontaneous speech recognition packages are still not available at a reasonable price, and natural conversational interaction is not yet available in conventional systems. Users were asked to repeat speech phrases to input the speech command, pausing 
between words in the discrete speech recognition system used. The implementation of a more spontaneous speech input system would have presented a set of problems for both the multimodal integrator and the practical management of the experimentation. Spontaneous speech would have presented difficulties of resolving what to say, and when to speak, which were not suited to the experimental design. This is a classical problem when unconstrained speech is used human-computer interaction (Robbe et al, 1997).

\subsubsection{Information situations arising from fusion}

When fusing the input modes of speech and two-dimensional gesture, a number of possible situations can arise in connection with the match (or mis-match) of the information from the two modes. In this thesis we restrict ourselves to three possibilities:

- The information from both modes contradicts

- The information from both modes agrees (partially or completely).

- The information from both modes is incomplete. The necessary information may be missing, preventing a conclusion being made. An extrapolation or guess may have to be made in these cases, or a request for extra information, in order to enable conclusion to be reached.

These possibilities are termed agree, disagree and incompleteness (or only one mode). The system therefore needed a strategy for coping with these possibilities.

In Section 4.5, it was pointed out that the speech recognition system provided arbitrary confidence ratings about its assessment. In like manner, the ranked-bid truth maintained blackboard system used in the gesture recognition system also provided ratings about the gesture conclusions (Section 5.7). These ratings are used in the multimodal integrator, as they provide a mechanism for choosing between information from modes, in the cases where ambiguity arises. Although the systems were independent, making any comparison arbitrary, it formed the basis of a simple algorithm for resolving the speech and gesture input information. 


\subsubsection{Representing Information}

In order to integrate the information from two independent systems into a single (multimodal) representation, a consistent method of analysing, choosing and combining information was required. A first step towards achieving this is the adoption of similar representations for the information from both sources. The representation needs to be rich enough to support different applications, as this has an effect on the nature, volume and type of information involved. An efficient, modular design is required which will also allow the accommodation of other information types when required, making the system re-usable and more application-independent.

An equivalent semantic form of input for each mode is also required, since the system is to be used for investigating redundancy between input modes. This ensures that redundancy can be easily identified across the distinct inputs, and can also be controlled and manipulated for experimental needs.

A method of representing information using slots was devised. The information from the speech system and the gesture system is categorised into slots. These independent slots are compared and combined with one another to create multimodal slots. This approach is similar to the natural language processing technique of conceptual dependency, a simple and elegant form of representation (Schank, 1972).

Schank's conceptual dependency framework represents every .action as being composed of component parts, which can fill a constant set of slots, regardless of the differences in the description of that action (Schank, 1972). This format of representation is a common theme in the integration of information from multiple modes. It can be seen in most of the work on integrating information from input modes (Billinghurst et al, 1996a; Billinghurst et al, 1996b; Koons et al, 1993; Nigay \& Coutaz, 1993; Vo \& Wood, 1996) so the technique has already been validated for holding the single, multimodal representation required. The method 

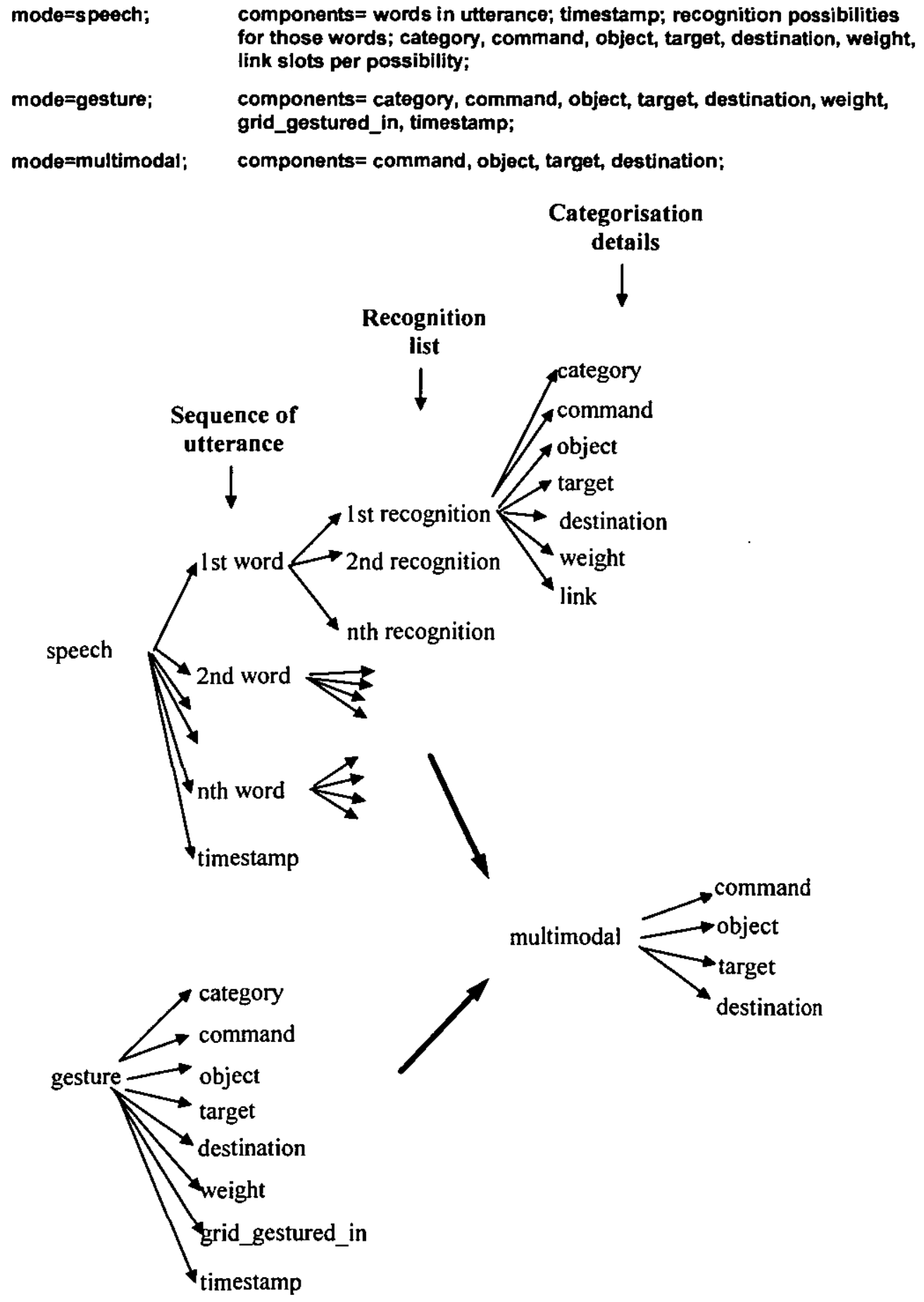

Figure 24: Slot structures for speech, gesture and multimodal information

The multimodal slots are filled in during the integration process, by comparing, combining and choosing from the speech and gesture slots. The three circumstances of modal agreement, modal disagreement, and modal incompleteness dictate the integration process. 


\subsubsection{Detailed Integrator Architecture}

System and context knowledge are used in the integration process. Information from the speech and gesture systems is processed to create representations of the information in appropriate forms.

\subsubsection{Discrete speech recognition system}

The commercial speech recognition package, DragonDictate, used for speech recognition, provides a recognition choice for that word, and if appropriate, a list of secondary recognition possibilities for that word. A percentage confidence for the recognised word, and estimates of confidence for the other recognition possibilities are also provided by the recognition software. The utterances may be one word only, or phrases of up to seven words.

The set of information for each word is processed further, within the context of the multimodal system, into the speech slot structure. Each recognition possibility is categorised against the pre-set system vocabulary, into one of four possible groups: commands, objects, grid numbers, link words (e.g. prepositions, definite and indefinite article) dependent on the value held in the category slot. This process is repeated for each word in the utterance.

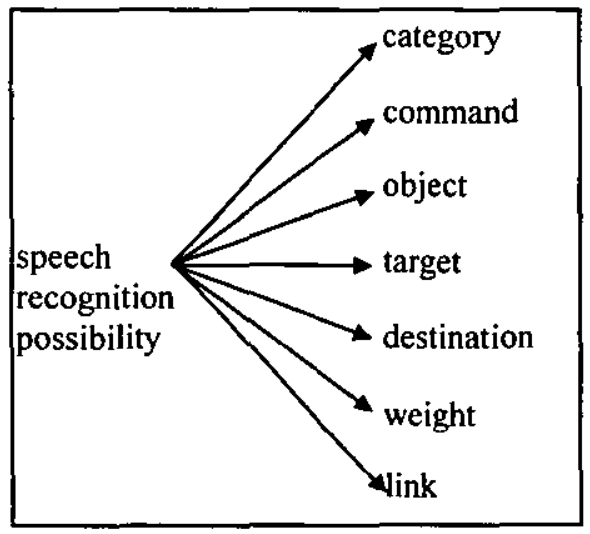

Figure 25: Available speech slots

After categorisation, each appropriate speech slot for that possibility is filled with the recognition value. Therefore a speech structure is produced for each spoken 
phrase, with a variety of different recognition possibilities completed for each word in that phrase.

\section{Categorising speech possibilities}

The categorisation sequence is:

$$
\begin{aligned}
& \text { phrase broken down into->component words } \\
& \text { each word has->recognition possibilities } \\
& \text { each recognition possibility is->categorised }
\end{aligned}
$$

The categorisation of the recognition possibility is achieved by comparing it with a list of predefined categories (command, object, grid number, link) and finding a match. The category value is then stored in the category slot of the speech structure. This process is therefore making use of task knowledge to perform the categorisation, and this effects the choices made by the speech interpreter to fill the slots. Following the categorisation of the recognition possibilities, the remaining slots of the structure are filled with codes representing: command, object, location (target or destination) or link information. A slot is left blank in a recognition possibility if it has not been categorised as being of that type (Figure 26).

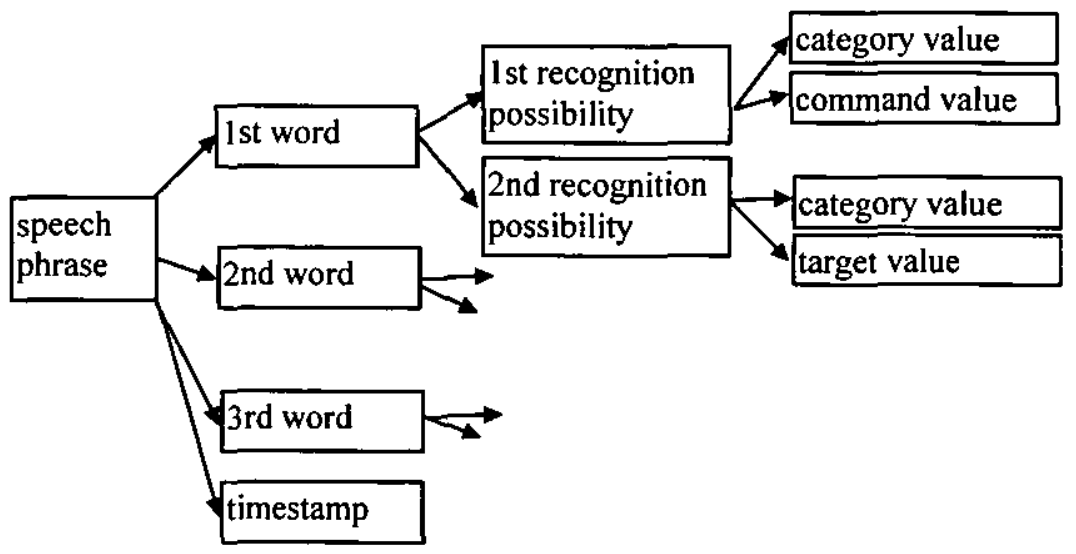

Figure 26: Structure of a speech phrase with filled slot values 
Therefore, the final structure for speech phrases can be represented more accurately as:

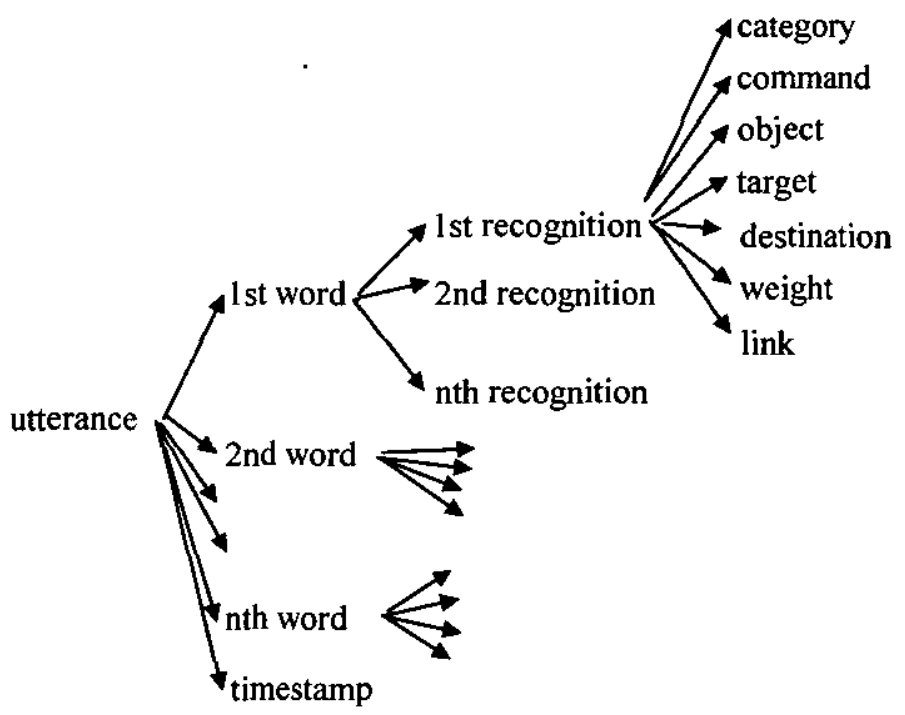

Figure 27: Utterance slot structure

Figure 27 shows the structure used to accommodate all the information from a spoken utterance, containing more than one word. Each recognition possibility of each word in an utterance was assigned a category, command, object, target and destination slot. Slots, noting the time that the speech utterance was recorded and processed, are also completed. This temporal information is used later in the multimodal integration process.

If the recognition possibility is categorised as a command word, the associated command slot is filled with that value. Similarly, if the possibility is classified as an object, the object slot is filled with that value. If the possibility is categorised as a link word, then the link slot is filled with the value. If the possibility was classified as a number, two situations are possible: the number is a target, or the number is a destination.

The difference between target and destination is determined by the value contained in the link slot of the previous word. If the link slot contains the word 
'from', then the number of the current slot is classified as a target number. If the link slot contains the word 'to', then the number of the current slot is classified as a destination number. Otherwise, if the link slot was empty, and the system could not determine if the number was a target number or a destination number, then both of these slots were filled with the value. Another attempt to determine the difference is then made later in the integration process. When a slot of either command, object, target, destination or link has been filled, the percentage confidence rating for that filled slot is placed in the associated weight slot.

This structure is illustrated in Figure 28 with an example speech phrase "Copy the Heart from 11", its recognition possibilities and categorised slot values. 


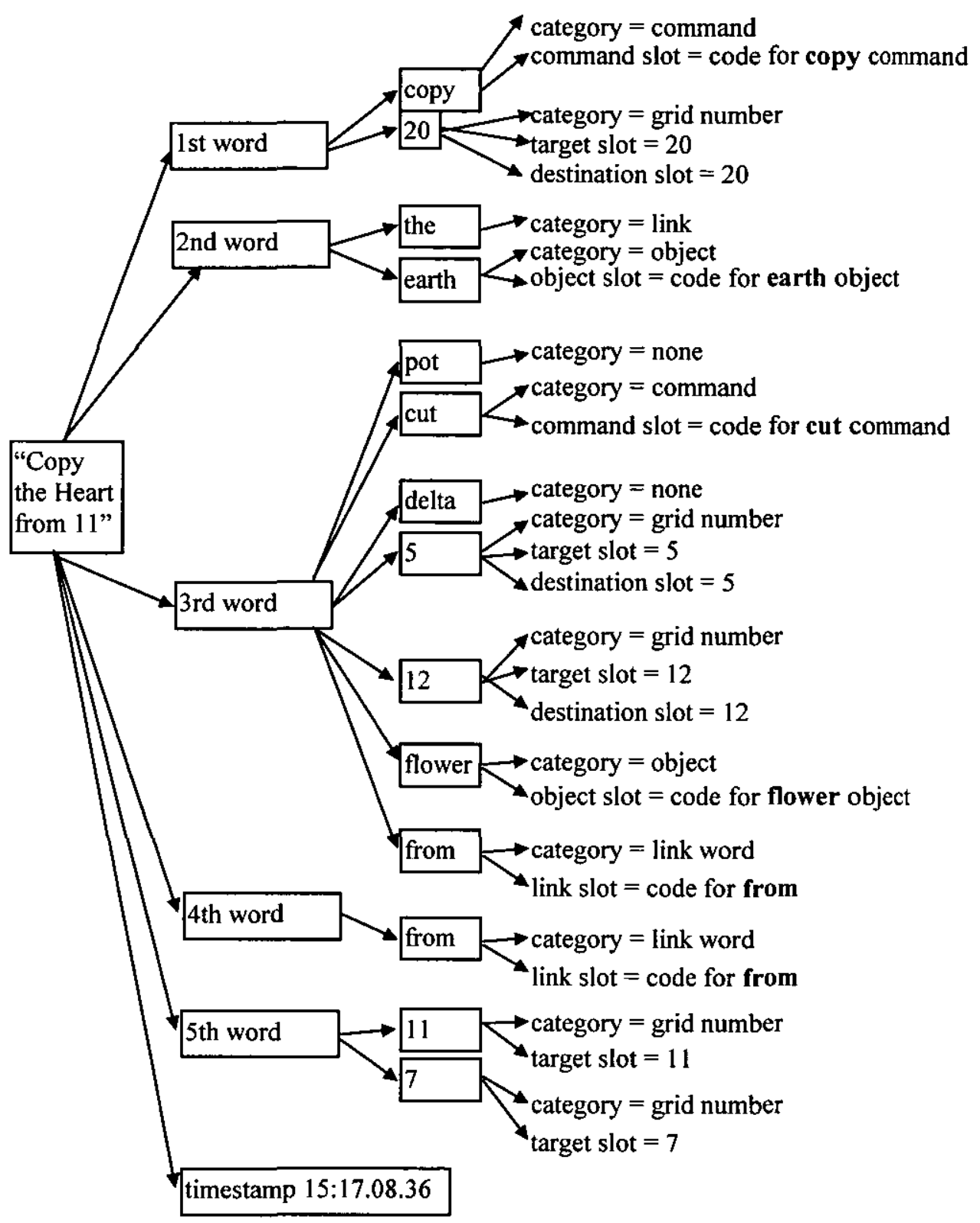

Figure 28: Illustrated speech phrase structure

Although only one slot out of those available (command, object, target, destination) is filled, the presence of all the slots is necessary to ensure that when combination and comparison with the gesture slots occurs, a consistent slot by slot comparison is possible. Although each recognition possibility cannot contain a complete set of filled slots, this may be a property of the whole utterance. The complete utterance structure contains the complete set of interpreted values for command, object and location information, as appropriate for that utterance. 

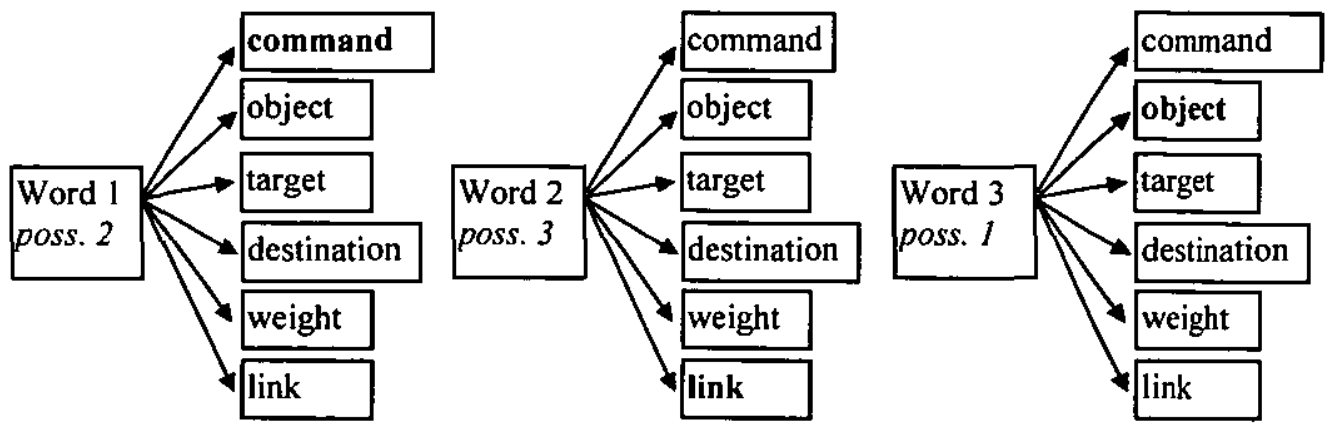

Figure 29: Distribution of values slots within words of an utterance

Figure 29 shows the possible distribution of category values, within the recognition possibilities of the words in a spoken utterance. The filled values are shown in bold. Therefore, when the utterance is viewed over all its component words, the necessary information for a complete interpretation is available across the words and recognition possibilities.

An example based on the same phrase of "Copy the Heart from 11" is shown in Figure 30 .
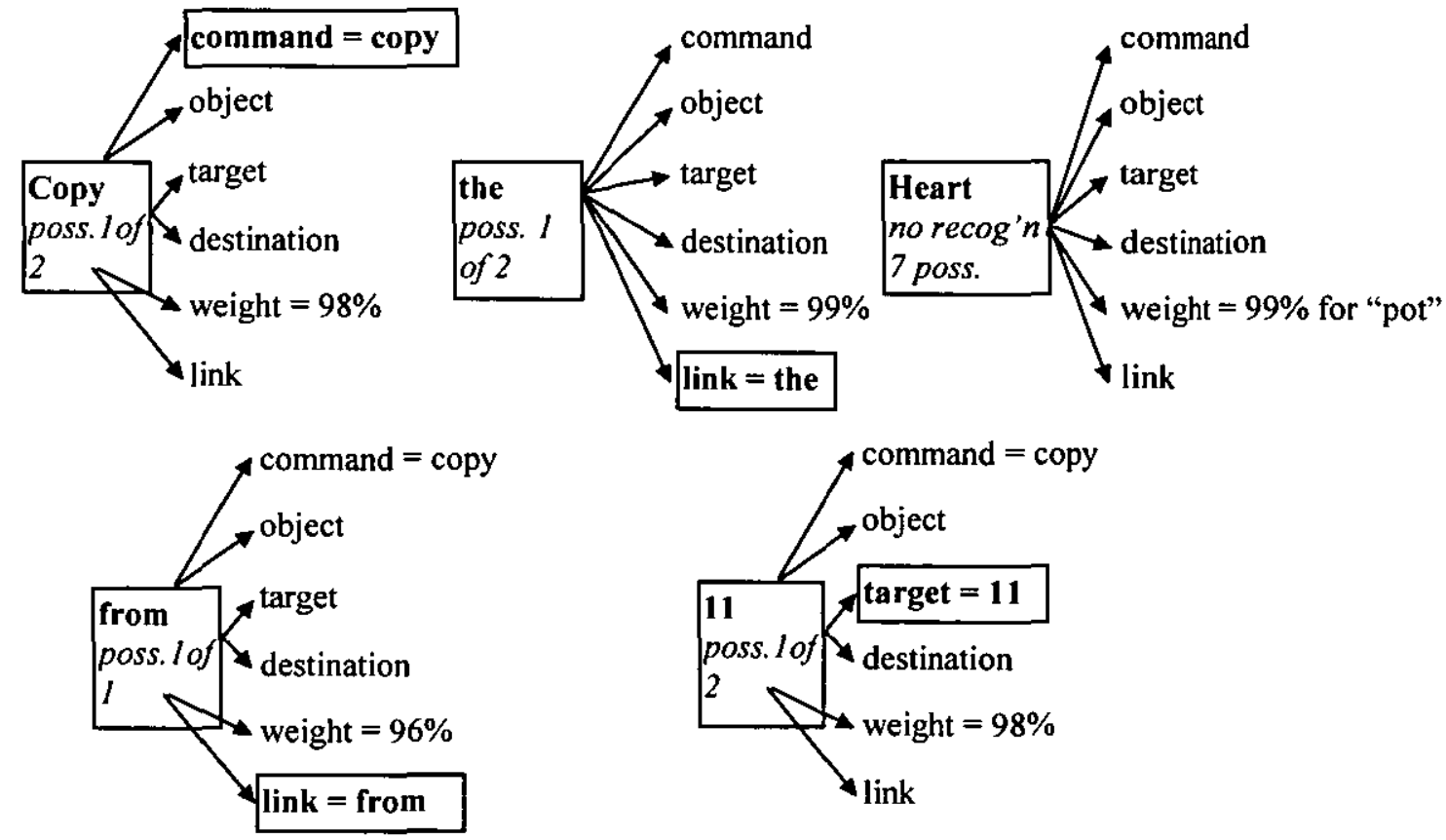

Figure 30: Distribution of recognition values in the phrase "Copy the Heart from 11" 
The complete interpretation of the phrase was therefore "Copy the - from 11", as the word "heart" was not interpreted by the system. The other recognition possibilities for "heart" can be accessed later, in the integration process, when the multimodal integrator will inspect the secondary possibilities and compare with the gesture information, if appropriate.

A similar, although simpler, structure is used for representing the gesture information.

\subsubsection{Touch \& Gesture recognition system}

As described in Chapters 4 and 5, the two dimensional touch and gesture recognition system used a truth maintained blackboard system (Hinde et al, 1989) to provide the gesture recognition conclusions. The raw co-ordinate data gained from the gestures, made on the touchscreen, was processed by the gesture system, which then forwarded the parameters based on this data to the truth maintained blackboard system (Section 4.4).

The blackboard system returns its conclusion about what it considers the gesture type to be, along with an estimate of confidence for that conclusion, since the knowledge bases in the system contain percentage ratings for each possible gesture rule embedded within them. Therefore, the blackboard system returns the necessary information to fill the command and weight slots of the gesture structure. 


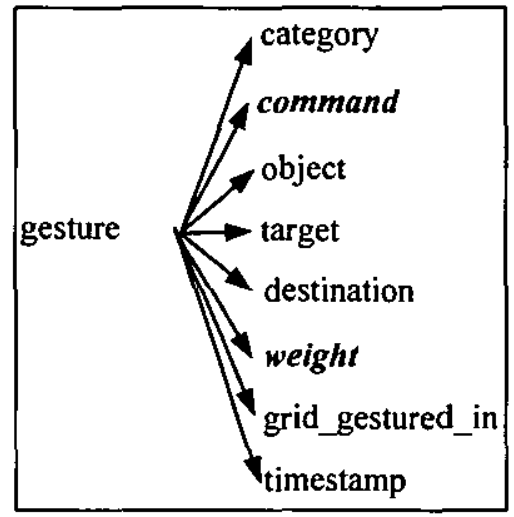

Figure 31: Slots of gesture structure

The gesture recognition system then provided the object and location information (target and destination), as well as the grid_gestured_in slot. The grid_gestured_in slot represents the area of the screen where the majority of the gesture had occurred, irrespective of the location of the target and destination points. A slot recording the time of the gesture execution is also completed. This time slot will be used in the integration process to determine context and relevance of speech and gesture to one another.

In contrast to the speech system, each gesture always has a command, object and location content. A gesture is only broken down in one stage into its component slots. There is no parallel with the speech structure, where an utterance was broken down into words, then into recognition possibilities, which are further decomposed into slots. For each gesture, all slots are filled, and each gesture is therefore on the same level as the utterance structure of speech, as the main representation level of the gesture input.

While the property of decomposing speech input into component words is necessary for interpretation, a similar treatment of the gesture input is more difficult to achieve. As two-dimensional gestures are inherently command-based (recall gesture properties from Section 4.3), decomposition into a 'command-less' or 'object-less' gesture is a more complex process than the equivalent treatment of speech input. As a result, this approach was not adopted for the experimentation, and is_not represented in the structures used in the system. This 
property of the speech and gesture input results in redundancy being controlled through the speech input, rather than through the gesture input, as the speech input can be varied in a way that gesture input cannot. Chapter 7 discusses the control of redundancy in the experimentation in greater detail.

\subsubsection{Framework Used}

The aim of the integrator is to complete the set of multimodal information slots, creating a multimodal 'phrase', in the structure introduced in the previous sections. The slots represent command, object and location information. In some cases the location is represented by one slot (either a target or destination) or by two slots (target and destination).

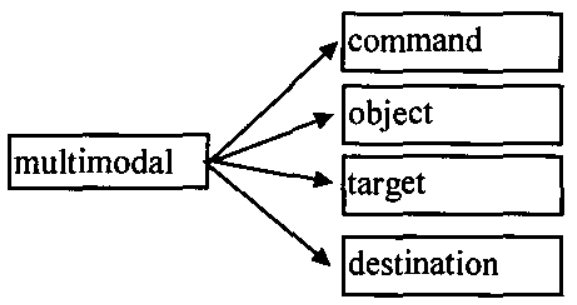

Figure 32: Multimodal slot structure

Once the speech and gesture input has been processed separately, the interpreted speech and gesture information is sent to the integrator for combination and validity checks. Three possible situations are dealt with by the integrator: where recognised speech and gesture information agree; where conflicts occur between the speech and gesture; and where only partial information is available across the modes. Speech and gesture timestamps are compared to ensure that the correct speech and gesture information is being considered together (by checking that the time gap between the inputs is acceptable, in our case, that gesture followed speech).

In all cases, at the start of the combination process, the first aim of the integrator is to determine the intended command. The first speech recognition possibility which had been categorised as a command is compared with the first gesture 
possibility. If the two slots agreed with one another, and are a valid value (nonzero), then this value is used to fill the multimodal command slot. If the two slots disagree with one another, then the one with the highest weighting is chosen to fill the multimodal slot. If only one option is available, preventing a comparison, e.g. only speech information available, no gesture available (because of misrecognition, non-recognition, non-categorisation etc.), then that option is chosen to fill the multimodal slot.

This same process is performed to fill the other multimodal slots, so object, target and destination slots are handled in the same way. A complete set of multimodal slots is produced, giving an overall interpretation of the original speech and gesture input.

This multimodal phrase is then checked for validity, and if true, the process stops. If the possibility is false, the next gesture choice is then combined with the current speech utterance, and this combination sent through the above process again. If none of the gesture choices make a valid combination with the given speech choice, the next speech choice is used. Thus, a simple linear search of the two-dimensional space is performed until a valid combination is found. 


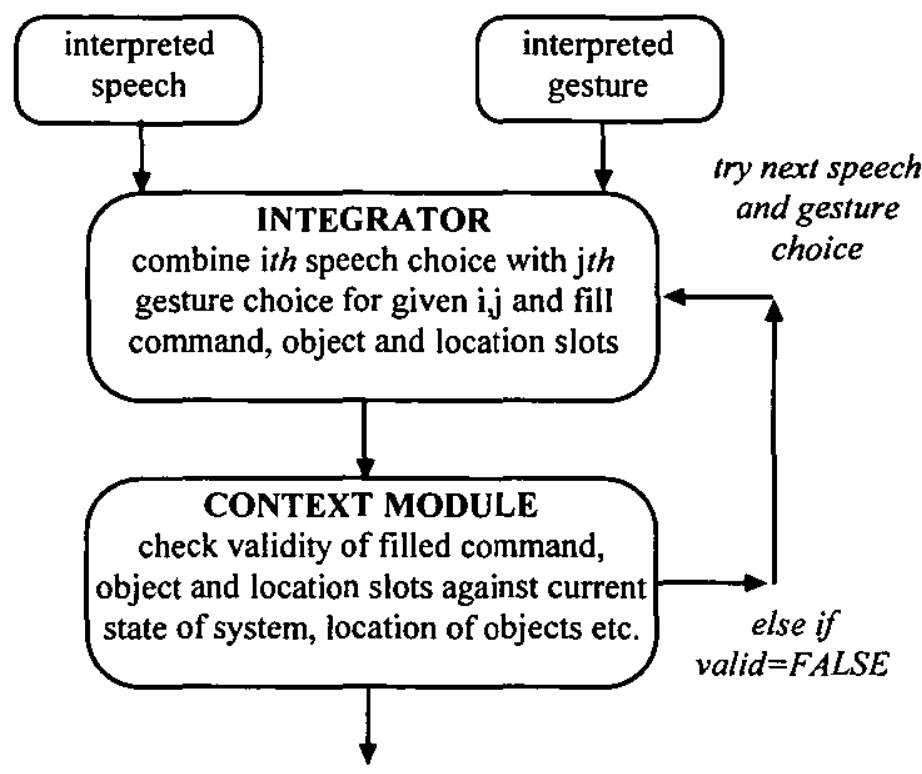

if valid $=$ TRUE, STOP, we have a valid multimodal interpretation

\section{Figure 33. Modal integration process}

The validity check is the process (referred to in Figure 33 as the Context module or task knowledge) where the validity of the value of each combination of information slot is checked against the current system knowledge. This consists of explicit and implicit system knowledge such as current object positions, the relevance of objects to commands (e.g. pastes are invalid if there is no object on the clipboard), and for commands, whether the location information is valid (e.g. moves must have two location information slots to be valid).

Thus, the integration of speech and gesture in the multimodal input system is performed by combining a speech choice (there were at most ten possibilities) with a gesture choice (again, there are most ten) until a valid combination is found which is consistent with the task knowledge.

\subsection{Summary}

Thus the integration of speech and gesture information into a single multimodal representation was achieved by the use of conceptual dependency type slot 
structures. These were used to represent all of the available information from the speech and gesture input, and generated the multimodal conclusion. This style of representation provides a modularity which can be added to in future, if additional modes are required. It also lends itself to changes in the type of information being fused, as the information types can be easily changed to accommodate different information types.

The basic algorithm of the integrator and its handling of three integration possibilities (agree, disagree, incomplete information) while effective (see Chapter 8), could have been improved upon further. Discussion of these possible improvements is presented in the conclusions, Chapter 9. 


\section{Chapter 7}

\section{Multimodal Experimental}

\section{Design}

\subsection{Introduction}

In this chapter the experimental design is described and the environment for experimentation is presented. The requirements for the environment are discussed, and in particular, the design to ensure redundancy across speech and gesture modes is presented. 


\subsection{Aims of experiment}

In Chapter 1, we listed our objectives:

- To probe the role of redundancy in multi-modal interfaces

- To determine levels of recognition success at which redundancy has some or best effect

- To analyse the relationship between redundancy and multimodal recognition rates

- To investigate if multimodal recognition rates are higher than separate unimodal rates. If this is the case, where does the gain in recognition success come from?

- To investigate how often redundancy of information reinforces meaning when two input modes agree

- To investigate how often redundancy of information resolves meaning when two input modes disagree

\subsection{Experimental Design}

The application has already been briefly described in previous chapters. It consists of a numbered grid on which coloured shapes are manipulated by a combination of speech and gesture (Figure 34).

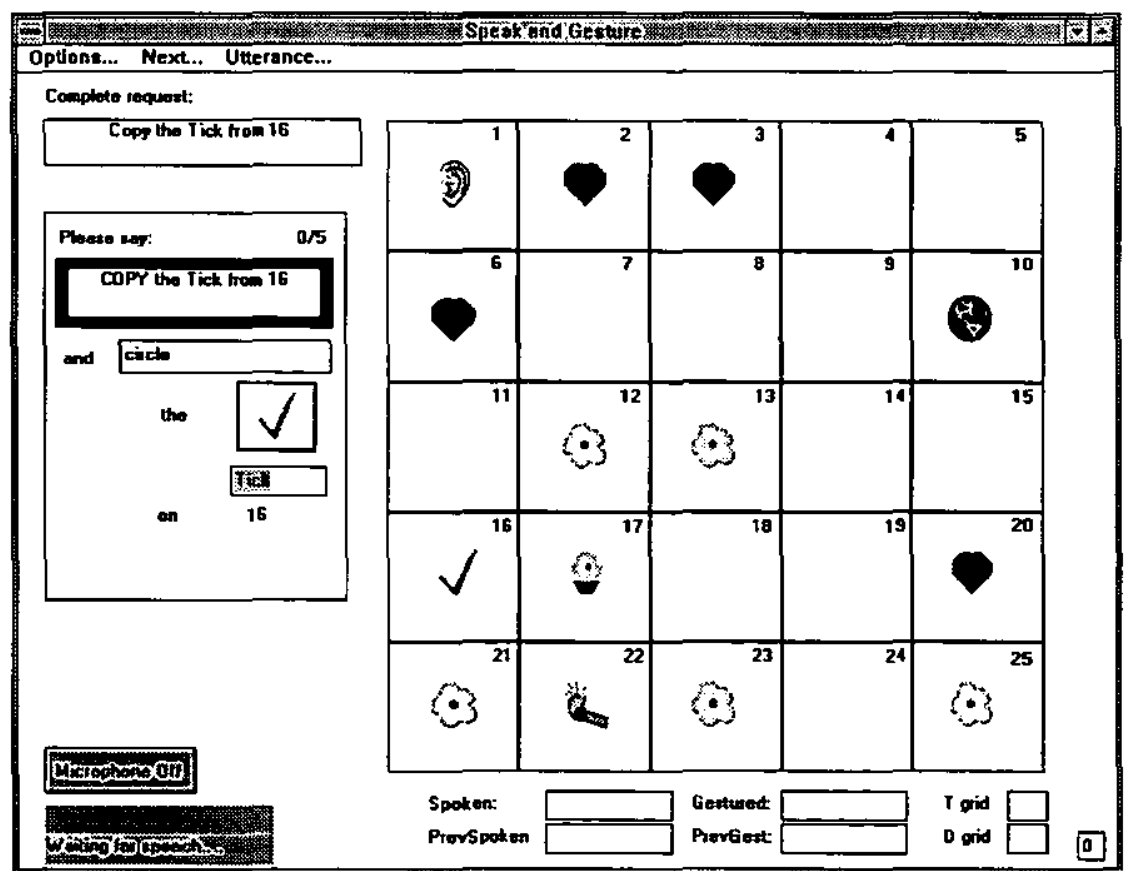

Figure 34. Experimentation interface 
Users are asked to carry out a number of requests on the grid, through speech and gesture input. An on-screen task guide generates the speech and gesture requests (see the dialog box on the left-hand side of the screen, Figure 34).

The four tasks which users are asked to complete are:

- $\quad$ cut [remove from grid]

- $\quad$ copy [store a copy on the clipboard]

-. paste [place from the clipboard to the grid]

- move [from one place on the grid to another]

These tasks are performed on the seven objects organised randomly in the grid (ear, earth, flower, flowerpot, heart, match, and tick) in locations numbered from 1 to 25 on the 5 by 5 grid.

\subsubsection{Automatic experimental requests}

One aim of experimentation was to analyse the effects of redundancy of information on multimodal recognition figures. Therefore an experimental method was required which would allow evaluation of recognition success. The recognition success of speech, gesture and multimodal inputs could only be measured if the intended speech, gesture and multimodal inputs were known.

Thus, if an utterance was recognised as the word "cut" and we are aware that the user was attempting to say "cut", then we have a recognition success. If an utterance is recognised as "pot" but the user was attempting to say "cut" then we have a recognition failure. Similarly for gestures, if a gesture was recognised as a circle and we know the user was attempting a circle gesture, then this is a recognition success. If a gesture was recognised as a tick when the user was attempting a circle then we have a recognition failure.

The attempted input from a user is logged so that it can be compared to the associated recognised input. It is more effective to automatically record users' attempts, rather than perform the complex and error-prone task of asking users to perform a particular speech and gesture combination, and then attempt to note the 
request manually. Therefore, an automatic task request system was devised, similar to the system used in the gesture experimentation (Chapter 5). Speech and gesture task requests were generated on-screen in random order (Figure 35), and recorded along with resultant user actions. In general, during the experimentation, all subjects followed the requests correctly. Only a few errors were made, on average 1 per person (a rate of considerably less than $1 \%$ ).

The speech and gesture requests were presented on-screen simultaneously (Figure 35). The user was required to perform the speech first, followed by the gesture. After the user had performed both the speech and gesture input, the system interpretation of the users' input could then be compared to the requested input. The request is regarded as the correct answer against which success is measured.

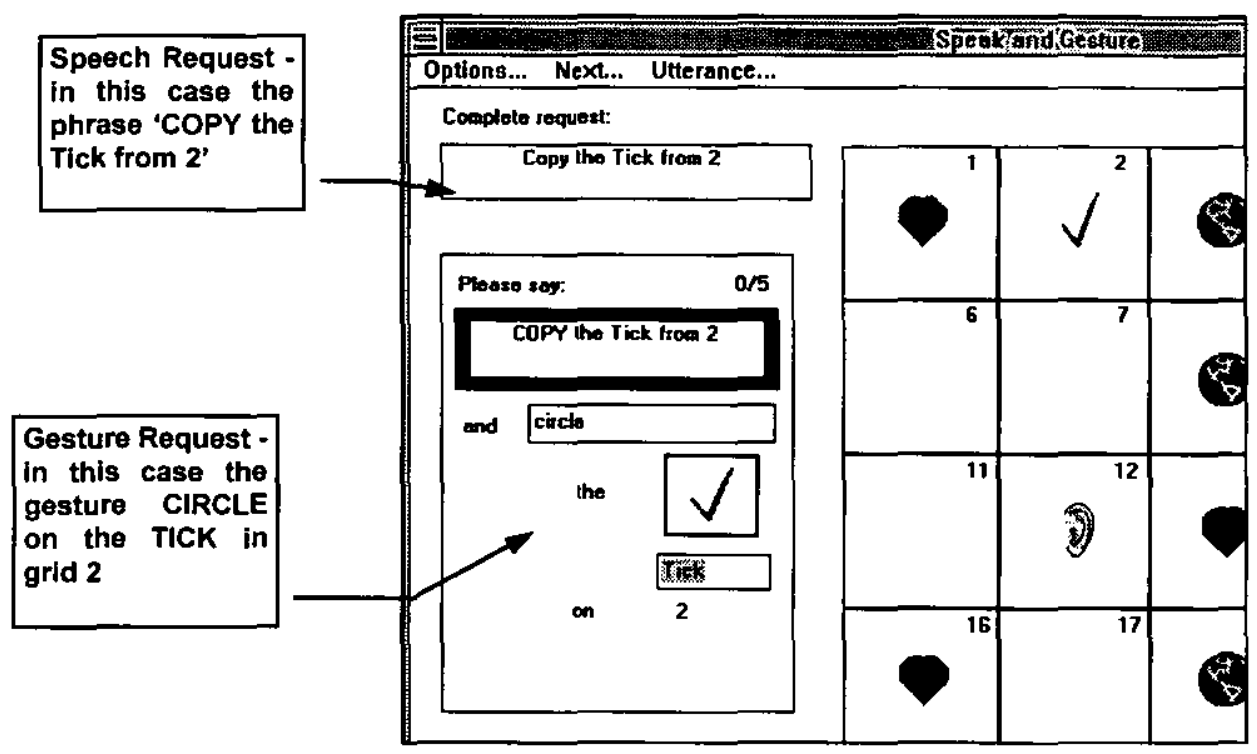

Figure 35. Request area of experimentation interface

\subsubsection{Error correction capability}

Errors made during speech could include the utterance of a word different from the requested word. Similarly a gesture could be made which differed from the request, in a different location to the requested location etc. Any significant errors made during speech could be corrected, by resetting the request and making users repeat an utterance. 
For instance, a laugh, sneeze, cough etc. could be detected by the microphone and processed as part of the utterance. Only the experimenter had control over whether an error should be corrected, users did not make this decision. If a significant error occurred during a gesture, such as a user accidentally touching the touchscreen before gesturing, the whole speech and gesture request could be repeated if necessary. However, in practice, these error correction options were rarely used as the occurrence of such errors was minimal.

\subsection{Design to Investigate Redundancy}

The system had to provide an environment for investigating redundancy, by allowing for the repetition of input information across modes. Therefore, an equivalent semantic form of input across the modes is required to ensure redundancy. In the system, three basic information fields must be filled for a complete input interpretation: command slot; object slot; and location slot(s). These slots are common to both the speech input and the gesture input.

Users are asked to follow action requests which were a combination of these slots of information, and the request is fulfilled through the joint inputs of speech and gesture. The speech input or utterances are single words or phrases of up to seven words generated by the system. In some cases, the speech request is equivalent to the complete action request, whilst at other times the speech request is only part of the complete action (e.g. 'Move the Heart'). Speech utterances were therefore divided into three categories: single, partial and complete utterances, representing various types of slot repetition. All gestures included the complete set of slots.

It was theoretically possible, in maximal cases, for the speech input to complete the multimodal slots, without reference to the gesture input. In all cases, it was possible in theory for the gesture input to complete the multimodal slots, without reference to the speech input. All gesture cases had the capability to provide this, as there was no partial information content in the gesture input. 


\begin{tabular}{|c|c|c|}
\hline Action:-2010 & GiticonviPgiste & \\
\hline Single Speech Request & $33 \%(1 / 3$ slots repeated $)$ & $25 \%$ (1/4 slots \\
\hline Partial Speech Request & $66 \%(2 / 3$ slots repeated $)$ & $75 \%(3 / 4$ slots $r$ \\
\hline Complete Speech Request & $100 \%$ (3/3 slots repeated) & $100 \%(4)$ \\
\hline
\end{tabular}

Table 10: Possible redundancy scores dependent on speech type \& action type

\subsubsection{Calculating recognition rates}

The requests and resultant user actions were recorded in requested slots (what was asked) and interpreted slots (the result). To calculate the recognition rates for speech alone, gesture alone and multimodal input (integrated speech and gesture), the values of the interpreted slots were compared with the values of the requested slots. As discussed in the previous chapter, interpreted speech input was used to fill speech command, speech object and speech location(s) slots. Similarly, interpreted gesture input processed by the truth maintenance system, filled gesture command, gesture object and gesture location(s) slots. The information from these speech and gesture slots was then used to generate multimodal command, object and location(s) slots in the multimodal integrator.

The automated task request system filled speech, gesture and multimodal task request slots. For cut, copy, and paste requests, three slots in the speech, gesture and multimodal knowledge base need to be filled: command, object and target. For move requests, four slots need to be filled: command, object, target and destination. Speech requests were, in some cases, intended to be incomplete (one, two, three or four slots filled). This allowed us to examine the concept of degree of redundancy between speech and gesture. When speech had the maximum slots filled, there was complete redundancy between speech and gesture.

A scoring system, by which recognition success was measured, was required which would take account of the variability in the number of slots involved in the requests and results. The number of slots actually requested in speech and gesture therefore governed the maximum possible score value of the speech and gesture requests. For instance, a speech request of the form 'Paste the Heart' includes only two requested slots, command and object, and the target slot is missing. If 
both 'Paste' and 'Heart' are correctly recognised, a comparison of interpreted against requested slots would find that the requested command and object equalled the interpreted command and object. This would give a score of $2 / 2,2$ slots requested, and 2 slots correctly interpreted. For that overall request, the speech score would therefore be $100 \%$. If only one slot had been correctly interpreted (e.g. 'Paste') the score would have been $1 / 2$, or $50 \%$.

The gesture and multimodal scores were also calculated on the same basis. For all of the 128 requests which each user performed, all speech, gesture and multimodal scores were calculated and then the average taken to give overall speech, gesture and multimodal recognition rates per user.

These main modal recognition rates represent the success of the interpretation of the input from that mode, for the associated task for that mode. Since the speech task is not always equivalent to the complete task, precise comparison of speech, gesture and multimodal task recognition rates should be done with caution. Modification of the modal speech recognition rates is therefore required (the gesture task and the resulting multimodal interpretation are always equivalent). To compare with regard to complete task interpretation, the speech recognition rates need to be calculated with a factor representing the percentage of the complete task that the speech task represented (i.e. the redundancy level). If the speech request represents $1 / 4$ of the total task, and achieves a recognition rate of, for example, $79 \%$, then the task recognition rate is $1 / 4$ of $79 \%(=19.75 \%)$, and the maximum rate is at most $25 \%$ in this case.

\subsubsection{Chosen repeated slots}

Given the four possible slots of command, object, target and destination $(\mathrm{C}, \mathrm{O}, \mathrm{T}$ and D) to be filled there was a total of $2^{4}-1=15$ possible combinations, detailed in Table 11. Based on the varying levels of redundancy, the speech requests used were one of the following nine possibilities: COTD, COT, COD, CO, CT, C, O, T, D. The associated redundancy levels relate to the commands that the speech phrases mapped to. For instance, the COT slot represented $100 \%$ redundancy for 
a CUT, COPY or PASTE command, but only represented $75 \%$ redundancy for a MOVE command. Similarly the $\mathrm{C}$ slot applied to all commands, representing redundancy levels of $25 \%$ for MOVE commands and $33 \%$ for CUT, COPY and PASTE commands. T slots were only repeated for CUT, COPY and PASTE while D slots only applied for MOVE commands.

\begin{tabular}{clc}
\hline Ref \# & $\begin{array}{l}\text { Possible slot } \\
\text { combinations }\end{array}$ & $\begin{array}{c}\text { Redundancy level } \\
\text { (Cut, Copy \& Paste) / Move }\end{array}$ \\
\hline 1 & COTD & $100 \%$ \\
2 & COT & $66 \% / 75 \%$ \\
3 & COD & $75 \%$ \\
4 & CO & $66 \%$ \\
5 & CT & $66 \%$ \\
6 & C & $33 \% / 25 \%$ \\
7 & O & $33 \% / 25 \%$ \\
8 & T & $33 \%$ \\
9 & D & $25 \%$ \\
10 & CTD & not used \\
11 & CD & not used \\
12 & OTD & not used \\
13 & OT & not used \\
14 & OD & not used \\
15 & TD & not used \\
\hline
\end{tabular}

Table 11: Chosen slot combinations

During design of the experiment the single, partial and complete speech requests were based around the use of four single-slot possibilities (slot numbers 6-9, Table 11), two of the two-slot partial possibilities, CO and CT (slot numbers 4 and 5), two three-slot possibilities, COT and COD (slot numbers 2 and 3) and the only four-slot possibility COTD (slot number 1 ).

This gave a range of redundancy levels between speech and gesture, two slots produced $100 \%$ redundancy, two at $75 \%$, two at $66 \%$, three at $33 \%$, and three at $25 \%$. Hence six possible combinations were omitted CTD, CD, OTD, OT, OD, and TD (slot numbers 10-15). 
The choice of speech slots was governed by three main goals:

- Minimise repetition between slots. For example, because two number slots were possible in move combinations, we judged the number slots to be similar to one another in information content, and did not want unnecessary repetition of information (e.g. CT and CD similar, so CD omitted).

- The speech requests were constructed to form natural phrases with appropriate definite and indefinite articles (the, a) and prepositions, the link words (from, to). Some combinations would not naturally form natural speech phrases, such as the OTD, OT, OD and TD slots (there is no command slot present).

- Balance the number of combinations that produce redundancy levels. For instance, only two combinations produce levels of $100 \%$ (COTD \& COT). Therefore, two combinations have been chosen which produce redundancy of $75 \%$ (COT \& COD; CTD omitted), two have been chosen which produce $66 \%$ (CO \& CT). Three combinations produced a redundancy level of $33 \%$, all of which were used $(\mathrm{C} 33, \mathrm{O} 33$, and $\mathrm{T} 33)$. Three combinations have also been chosen for the $25 \%$ redundancy level, C25, O25 and D25.

The chosen slots and the final associated redundancy levels are shown in Table 12.

\begin{tabular}{ll}
\hline Repeated Slots & Redundancy level \\
\hline C25, O25, D25 & $25 \%$ \\
C33, O33, T33 & $33 \%$ \\
CO, CT & $66 \%$ \\
COD, COT75 & $75 \%$ \\
COT100, COTD & $100 \%$ \\
\hline
\end{tabular}

Table 12: Chosen repeated speech slots and associated redundancy levels

The choice of redundancy levels assumes that different individual slot types merit the same weight. The individual command slot is given the same redundancy level $(25$ or $33 \%$ ) as the individual number slot (destination at $25 \%$ or target at $33 \%$ ). The results may demonstrate that the levels need to reflect the different information types, as there are differences in the recognition success between words and numbers.

\subsection{Experimental Format}

Eight users were involved in a pilot experiment, thirty users in the main experiment. All subjects used computers regularly, but had no significant experience of using either speech or gesture input technology. Each user was 
asked to follow the on-screen task guide which generated (in a pseudo-random order and combination) speech and associated gesture instructions. The task required users to manipulate coloured objects around a grid using speech and gesture (recall the application screen in Figure 34, p145).

Before beginning the shape game, users were asked to train the speech software on a selection of words associated with the application. This gave users the opportunity to familiarise themselves with the use of the microphone and speech recognition. The training also provided vocabulary files that the system used in recognising speech during the main experiment. The training of vocabulary by users was used to improve the possible speech recognition rates that could be achieved by the software. Without training, the speech recognition is unreliable, giving poor recognition results.

In total, each user was asked to perform 128 combinations of speech and gesture, split over two sessions to prevent fatigue. Speech input was through the microphone with discrete voice recognition software, whilst gesture input was made with a touchpen on a pressure-sensitive touchscreen. No time restrictions were imposed upon users although other restrictions were in force, as described below.

Each combination of speech and gesture required that speech be performed before the gesture. Further, as the voice recognition software was discrete, users were asked to pause slightly between each word in an utterance. A word count in the guide window provided feedback about the number of words uttered and received by the system.

Users were given instructions before the experiment on these restrictions. They also received instructions on the predefined gestures and the associated commands of cross for cut, circle for copy, tick for paste and line for move, (where cut, copy and paste are defined as in standard text editing meaning). 


\subsection{Integration Example}

During each experimental session, the log files record the speech and gesture input which users are attempting, and the recognition results of this input. The files contain detailed information of the speech, gesture and multimodal slots, and the attempts in the integration process as well as the final multimodal phrase produced are noted.

Examination of this information gives insight into the operation of the multimodal integrator, its success, and also generates suggestions for improving the algorithm. The following example of the integration process is from the first subject of the experiment, attempting to speak the phrase "Move the Heart from 4" and make the gesture Move the Heart from 4 to 21 (a line of positive gradient).

\begin{tabular}{lllll}
\hline Word number & Recognition choice & Result & Category & Confidence \\
\hline First & 1st & 2 & grid number & $99 \%$ \\
& 2nd & to & link word & $99 \%$ \\
& 3rd & grid & link word & $97 \%$ \\
& 4th & 9 & grid number & $37 \%$ \\
& 5th & move & command & $36 \%$ \\
\hline Second & 1st & the & no category & $96 \%$ \\
& 2nd & 5 & grid number & $33 \%$ \\
& 3rd & earth & object & $16 \%$ \\
\hline Third & lst & pot & no category & $98 \%$ \\
\hline Fourth & lst & from & link word & $97 \%$ \\
\hline Fifth & Ist & 4 & grid number & $94 \%$ \\
\hline
\end{tabular}

Table 13: Speech recognition results

The gesture recognition results are:

\begin{tabular}{clll}
\hline $\begin{array}{c}\text { Gesture possibility } \\
\text { number }\end{array}$ & Command Result & Confidence & $\begin{array}{l}\text { Object and Location } \\
\text { slots }\end{array}$ \\
\hline 1 & Positive gradient line & $68 \%$ & heart, at location 4, 21 \\
2 & Positive gradient line & $64 \%$ & heart, at location 4,21 \\
3 & Positive gradient line & $63 \%$ & heart, at location 4, 21 \\
4 & Positive gradient line & $49 \%$ & heart, at location 4, 21 \\
5 & Tick & $45 \%$ & heart, at location 4 \\
6 & Positive gradient line & $45 \%$ & heart, at location 4, 21 \\
7 & Positive gradient line & $30 \%$ & heart, at location 4, 21 \\
8 & Positive gradient line & $27 \%$ & heart, at location 4, 21 \\
9 & Circle & $25 \%$ & heart, at location 4 \\
\hline
\end{tabular}

Table 14: Gesture recognition results 
The combination attempt is:

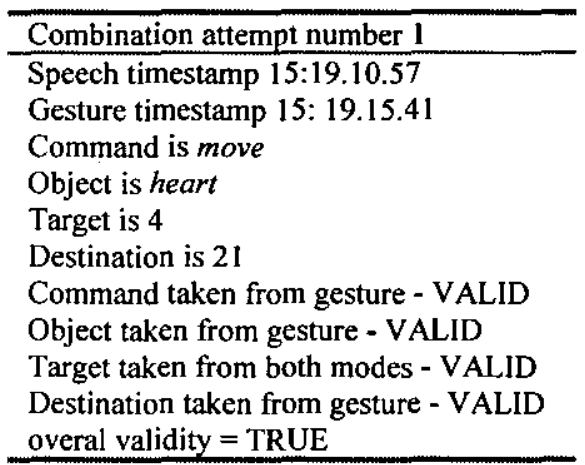

The integrator has found a valid combination on the first attempt, using the first gesture recognition possibility and the first speech recognition possibility. The integrator has taken the multimodal command and object from gesture, as gesture was the only mode to contain interpreted command and object information. Speech has not interpreted the command word until the fifth possibility of the first word, and no object slot was interpreted ("heart" was recognised as "pot"). The speech contained interpreted target information, as did the gesture, and the values agree. Only the gesture could provide the destination information in this case.

\subsection{Summary}

This chapter has presented the multimodal experimental design. The error correction capability of the system has been described, and the calculation of the recognition rates has been discussed. The design of the system to investigate redundancy has been presented, to ensure that variable redundancy is present between the modes. An example of the multimodal integrator operating in the application has also been described. 


\section{Chapter 8}

\section{Multimodal Experimental}

\section{Results}

\subsection{Introduction}

In this chapter the results from the multimodal experimentation are presented. The data from the final experiment is analysed for significant results and patterns according to the expectations and trends set out at the beginning of the experimentation. Descriptive statistics are shown where appropriate and the reasons for use of applicable statistical tests are described. Significance levels are also shown where appropriate and further analysis, where useful, is presented.

\subsection{Expectations}

From the experiment, three main results were expected. It was expected to find that the multimodal recognition rates would be higher than speech or gesture _recognition rates alone. It was also expected to find that there would be a 
correlation between the coarse calculated redundancy levels and the achieved multimodal recognition rates. Finally, it was expected to find that within the coarse redundancy levels, the differing slot repetition types would give distinct multimodal results for each category. From these results we expected to identify where the multimodal gain actually occurred and hypothesise on guidelines for multimodal integration.

\subsection{Initial Experiment}

An initial experiment with eight subjects was conducted to test the operation of the system and to test its effectiveness. Two users of this initial version demonstrated that it was possible, in certain circumstances, for the speech input to 'queue' up in the speech input buffer. As speech recognition is a discrete process, each word is spoken with a slight pause before the next word, whilst the system processes that word. Any noise is captured by the microphone and relayed to the system, which then attempts to process and recognise the noise, assuming it is speech. In this initial version of the system, the microphone was constantly switched 'on'.

The experimental system expects to receive a specific number of words per speech request. In the case of the speech request being 'Move the Heart to 11', the system expects to receive five words. If the utterance is actually "Move the [cough] Heart to 11", the system stops processing that utterance after the word "to", as this is the fifth word. The number " 11 " is lost for this speech request, but is stored in the speech buffer and processed as if it is the first word of the next speech request. The speech input has 'queued' up because of the additional [cough] input, and any additional noises made between speech utterances are also added to the speech buffer for processing. While the speech timestamps helped resolve which speech and gesture pairs belonged together, the additional processing of these extra noises and words delays the overall processing of the intended speech. It was decided to adapt the final experimental system so that these extra noises would not be captured, by switching the microphone off between utterances. 
The speech recognition figures for the two previously mentioned users, whose usual way of speaking included lots of sighs and tongue-clicking between words, ( 2 and 6 in Table 15) were lower than expected. It is interesting to note that their corresponding multimodal recognition rates are at similar levels to the other subjects, with subject 2 actually giving the highest overall multimodal recognition figure. The multimodal recognition rates in these two cases might have been expected to show significant differences from the other subjects in the experiment. This result is interesting because it shows that even when the speech input recognition is poor, multimodal recognition rates are higher than either the speech or gesture recognition rates alone.

The percentage recognition rates, shown in Table 15 and plotted in Figure 36, represent the average figures from each user, where interpreted information was compared with the requested information. For each task, the recognition rate (for speech, gesture and multimodal) is calculated by dividing the number of slots correctly recognised across the tasks by the total number of slots across the tasks. Gesture and multimodal recognition rates are calculated over the same task content. Since the speech input was controlling the redundancy between modes, the task content in the speech varied. The average speech recognition rates shown are therefore calculated over a smaller number of slots, and thus over different task content. Therefore, comparison of the overall rates provides a general view of system performance, but precise conclusions can only be made with detailed comparison in each redundancy level. Only the general comparison is presented for the initial experiment as the sample size of eight subjects is fairly small, and scope for significant conclusions is limited.

The overall gain percentage in the multimodal recognition rates over the gesture recognition rates is also shown. The multimodal gain represents the improvement made to the gesture recognition figures by the presence of additional speech information. 


\begin{tabular}{|c|c|c|c|c|c|c|c|c|}
\hline (USerf & II & 2 & $\overline{3}$ & $\bar{Q}$ & 5 & 6 & 9 & 8 \\
\hline Speech Recognition \% & 55.1 & 29.6 & 60.2 & 63.8 & 48.6 & 38.1 & 53.6 & 65.7 \\
\hline Gesture Recognition \% & 78.4 & 81.5 & 82.8 & 59.6 & 76.7 & 75.9 & 76.8 & 73.5 \\
\hline Multimodal Recognition \% & 82.7 & 89.0 & 88.7 & 74.1 & 85.1 & 79.1 & 83.3 & 81.4 \\
\hline$\%$ Gain & 4.3 & 7.5 & 5.9 & 10.3 & 8.4 & 3.2 & 6.5 & 7.9 \\
\hline
\end{tabular}

Table 15: Percentage Speech, Gesture and Multimodal Recognition Figures for 8 users

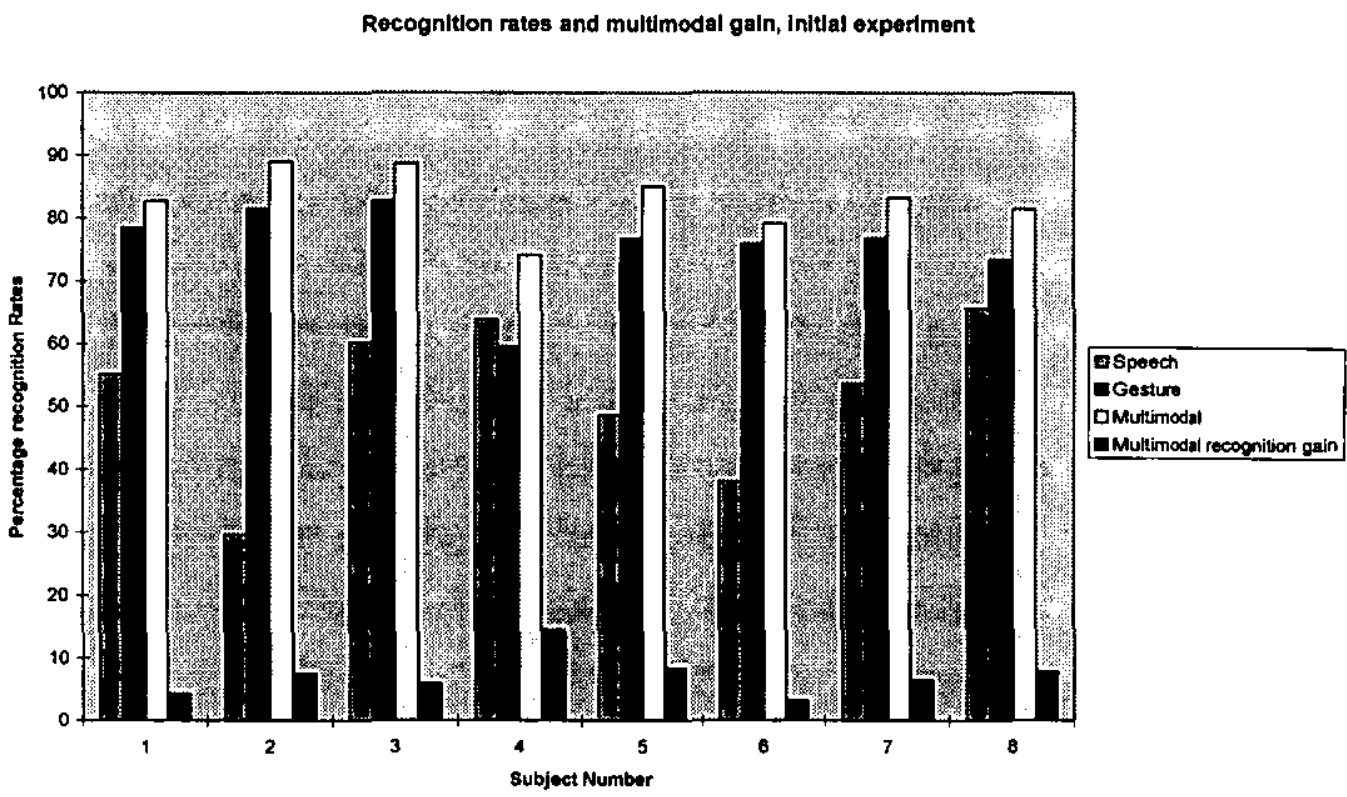

Figure 36: Plot of Speech, Gesture, Multimodal Recognition Rates, with multimodal gain shown, 8 subject experiment

For each user, the multimodal recognition rate was always higher than the gesture recognition figure. Over all eight users the average speech recognition rate is $51.8 \%$, the gesture recognition rate is $75.7 \%$ and the average multimodal recognition rate is $82.9 \%$. The descriptive statistics for these figures are shown in Table 16.

\begin{tabular}{llllllll}
\hline Vagriable & Mean & Sta.Dev & Variance & Min. & Max & N \\
\hline Speech & 51.84 & 12.61 & 158.95 & 29.6 & 65.70 & 8 \\
Gesture & 75.65 & 7.15 & 51.09 & 59.60 & 82.80 & 8 \\
Multimodal & 82.93 & 4.93 & 24.29 & 74.10 & 89.00 & 8 \\
\hline
\end{tabular}

Table 16: Descriptive Statistics for eight subject pilot experiment

To prove this difference between the means of speech, gesture and multimodal results is significant, an ANOVA might have been performed. However, the 
variances in the samples violate the assumptions for an ANOVA test, namely that the largest variance is no more than twice as large as the smallest sample variance. As a consequence, a non-parametric test was applied since it requires less assumptions about the data (Moore, 1995; Norusis, 1993). A test for several independent sample means, a Kruskal-Wallis 1-Way Anova was performed, as shown in Figure 37.

\begin{tabular}{|c|c|c|c|c|c|}
\hline \multicolumn{6}{|c|}{$\begin{array}{ll}\text { Kruskal-Wallis 1, SCORE by } & \text { SODE } \\
\text { Way Anova } & \text { MODE }\end{array}$} \\
\hline $\begin{array}{l}\text { Mean Rank } \\
4.88 \\
13.50 \\
19.13\end{array}$ & $\begin{array}{l}\text { Cases } \\
8 \\
8 \\
8 \\
24\end{array}$ & $\begin{array}{l}\text { MODE }=\text { Speech } \\
\text { MODE }=\text { Gesture } \\
\text { MODE }=\text { Multimodal } \\
\text { Total }\end{array}$ & & & \\
\hline $\begin{array}{l}\text { Chi-Square } \\
16.4850\end{array}$ & $\begin{array}{l}\text { D.F. } \\
2\end{array}$ & $\begin{array}{l}\text { Significance } \\
.0003\end{array}$ & $\begin{array}{l}\text { Corrected fo } \\
\text { Chi-Square } \\
16.4850\end{array}$ & D.F & $\begin{array}{l}\text { Significance } \\
.0003\end{array}$ \\
\hline
\end{tabular}

Figure 37: ANOVA table for 8 subject pilot experiment

It can be seen that multimodal rates have a higher average rank than the gesture rates. Therefore the observed significance level of $\mathrm{p}=0.0003$ suggests that the mode does have a bearing on the recognition scores, and the multimodal rates are significantly higher than gesture rates at $\mathrm{p}<0.001$.

We conclude that the average multimodal recognition rate is statistically significantly higher than the gesture recognition rates, and the speech recognition rate. The pilot experiment has shown the usefulness of the approach. Therefore a full study involving 30 users was performed.

\subsection{Main Experiment}

This section presents the results from the thirty subject experiment. Comments on the format of the experiment, subjects used, errors made by subjects and initial expectations at the outset of analysis, are covered. Raw data can be found in appendices, indicated as appropriate in the text. 


\subsubsection{Format}

The format for this experiment was as described in Chapter 7. Analysis of the data from thirty users was performed. A revision to the experimental design ensured that speech input could not "queue up", as the microphone automatically switched off between utterances. Any noises made between utterances were not picked by the speech system to distort the input (recall the poor speech recognition rates from the initial 8 subject experiment described in Section 8.3, where speech "queued up").

The data from each user was analysed for the following properties (across redundancy levels):

- average speech recognition figures

- average gesture recognition figures

- average multimodal recognition figures

- where speech and gesture modes agreed, the percentage of these cases leading to the correct multimodal conclusion

- where speech and gesture modes disagreed, the percentage of these cases leading to the correct multimodal conclusion

- relationships between which information slots were repeated (the redundancy) and overall multimodal recognition success

\subsubsection{Subjects}

Thirty subjects were used for the main experiment. All had experience of using computers, ranging from simple word processing skills to experienced use. None of the subjects had used speech or gesture input technology to any significant degree. Subjects had a wide variety of accents, with 23 having British accents, while 7 subjects had non-British accents. Since the speech recognition software was optimised for British accents, it might have been reasonable to expect lower recognition figures for those subjects with non-British accents. As the results show in the next section however, there was not a significant difference in the speech success figures between the British versus non-British speakers. (This is 
in contrast to the findings of Oviatt, who demonstrates that 'native' versus 'nonnative' speakers gave significantly different speech results in a multimodal environment (Alty, 1998)).

\subsubsection{Preliminary experimental observations}

\subsubsection{Errors made by subjects during interaction}

During interaction some users did become confused, performing actions contrary to those requested. For instance, in some cases where the overall action was Move the Heart from 6 to 9, some users performed the requested gesture backwards and so gestured from grid 9 to 6 , when the correct gesture would have been from grid 6 to grid 9 . One subject had particular difficulty in performing the gesture as requested, and repeatedly confused gestures between tasks. Where the requested gesture was a circle, for the copy task, the subject frequently performed a move task, with a line gesture. The effects of these errors were not corrected during gesturing and remained in the data.

\subsubsection{System performance}

Command and object input was handled well by the system, both in speech and gesture. However, the recognition and interpretation of number input caused the system more difficulty in the interpretation. This affected the overall execution of tasks in the application.

\subsection{Summary of Results}

\subsubsection{Results by subject}

First, the gesture and multimodal recognition rates are displayed per subject. This provides an overview of the general performance of the system. The average recognition rates are calculated, per mode and per subject, by dividing the number of slots correctly recognised by the total number of possible slots that could have been recognised: 


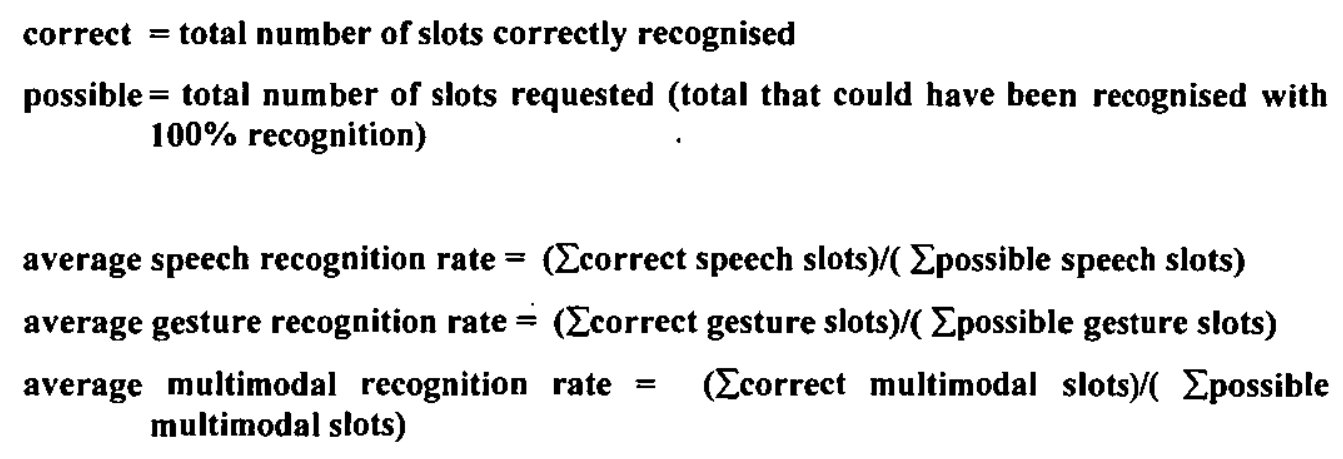

Since speech input is made over varying levels of task content: single word input, partial phrase input, and complete phrase input (which represent the single, partial, and complete redundancy levels in the system (Section 7.4)), the average recognition rates are not directly comparable with the gesture and multimodal rates.

For example, a speech recognition rate of $100 \%$ might refer to the correct recognition of a single word (e.g. 'Cut'), while in other cases a recognition rate of $100 \%$ could refer to the correct recognition of a complete phrase ('Copy the Heart to 4', 'Move the Fire from 6 to 12 '). Gesture and multimodal rates, in contrast cover the complete task. Since the number of cases of speech rates which cover the complete task are lower, this speech data is likely to have higher standard deviations and we therefore do not compare it with the gesture and multimodal rates at this stage.

Average gesture and multimodal recognition rates are in Figure 38 for each subject. 


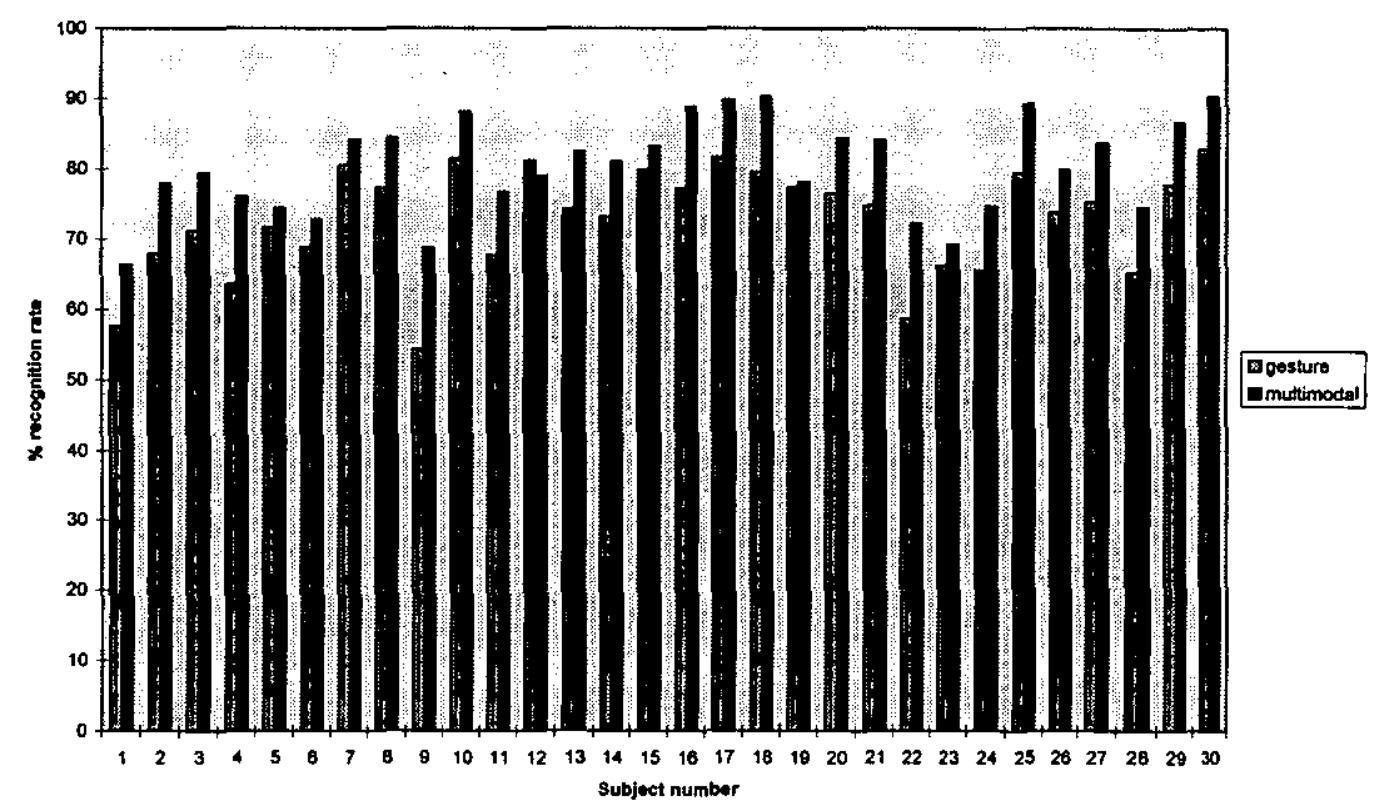

Figure 38: Comparison of gesture and multimodal recognition rates from 30 subjects

These results show that 29 out of 30 subjects generated average multimodal recognition rates which were higher than the gesture rates.

Inspection of the gesture recognition rates show that they vary from approximately $55 \%$ to $80 \%$. This variability in the performance of the gesture recognition per subject was expected, based on the earlier gesture experiments conducted (Chapter 5). Observation of subjects during experimentation had shown that individual variations, in the way gestures were performed, affected the results produced. Whilst the use of rule induction on the data, compensated for the variability by producing general classification models, extreme variations in gesture input can be expected to defy these classifications.

Boxplots have been chosen to display the data in the following sections. A boxplot is a way of showing the distribution and variability of data, by plotting summary statistics such as the median, percentiles, outliers and extreme values.

A dataset is displayed as a box and the median is the centre value in a dataset, shown as the centre line through the box. Half of the data values lie below this 
level, and the other half lie above. The percentiles (or quartiles) represent the middle half the data, the first quartile (25th percentile) being one quarter of the way through the data, and the third quartile (75th percentile) is three-quarters of the way through the data. Outliers are defined as values which lie more than 1.5 box lengths from the 25th or 75th percentile, and extreme values are those which lie more than 3 box lengths from the 25th or 75th percentile (plotted as circles and asterisks respectively). The bottom line (or whisker) indicates the smallest observed value that is not an outlier. Similarly, the top line indicates the largest observed value that is not an outlier.

The averages of all the subject gesture and multimodal recognition rates is shown in the boxplot of Figure 39.

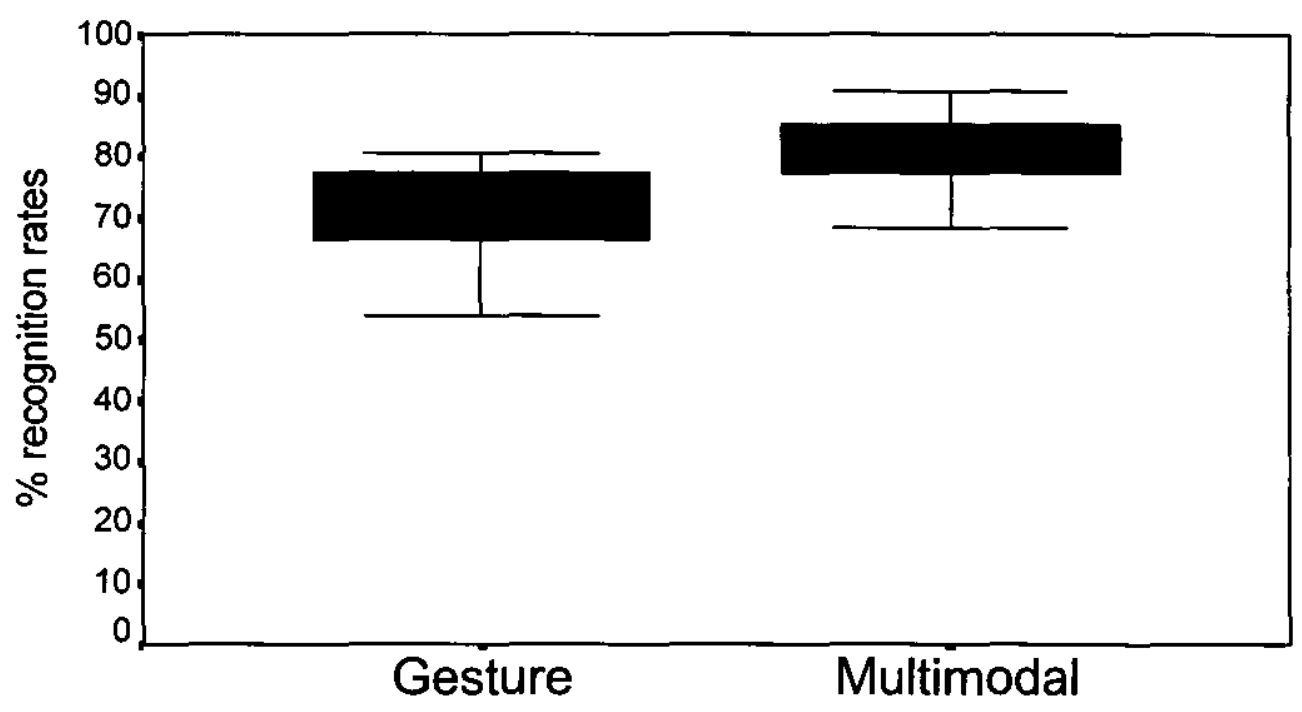

Mode

Figure 39: Boxplot comparing average gesture and multimodal recognition rates

The boxplot indicates that in general, the multimodal recognition rates gained from each subject are higher than the individual gesture rates. The average gesture rate is $72 \%$ and the average multimodal rate is $82 \%$. While these figures broadly indicate that the multimodal interpretation is an improvement on the 
gesture interpretation, detailed analysis according to the redundancy levels is required.

\subsubsection{Results by redundancy levels}

The speech, gesture and multimodal rates are displayed according to two main factors:

- $\quad$ Task type

$\diamond$ cut, copy and paste tasks (all containing three component slots: command, object and one location)

$\diamond$ move tasks (containing four component slots: command, object and two locations)

- $\quad$ Redundancy level

$\checkmark$ results from complete redundancy between speech and gesture input are shown (the $100 \%$ redundancy cases which were the complete speech phrase inputs)

$\diamond$ results from partial redundancy between speech and gesture input are shown (the cases of $66 \%$ and $75 \%$ redundancy, the partial speech phrase inputs)

$\checkmark$ results from single redundancy between speech and gesture input are shown (the cases of $25 \%$ and $33 \%$ redundancy, the single speech inputs)

Presentation of the results in this format allows precise comparison of recognition rates in each redundancy level. The rates can be taken as verbatim performance figures for a redundancy level within a task. Additional analysis categorised by all redundancy levels can be performed following these general results, to reveal if different information content has affected the recognition rates achieved.

Standard one-way ANOVAs are also performed on the speech, gesture and multimodal recognition rates, where appropriate, to determine whether differences in the means are significant.

\subsubsection{Complete redundancy results}

The speech, gesture and multimodal recognition rates are plotted in Figure 40 for the $100 \%$ redundancy level across all actions (where the amount of task 
information in speech input is equivalent to the amount of information present in the gesture).

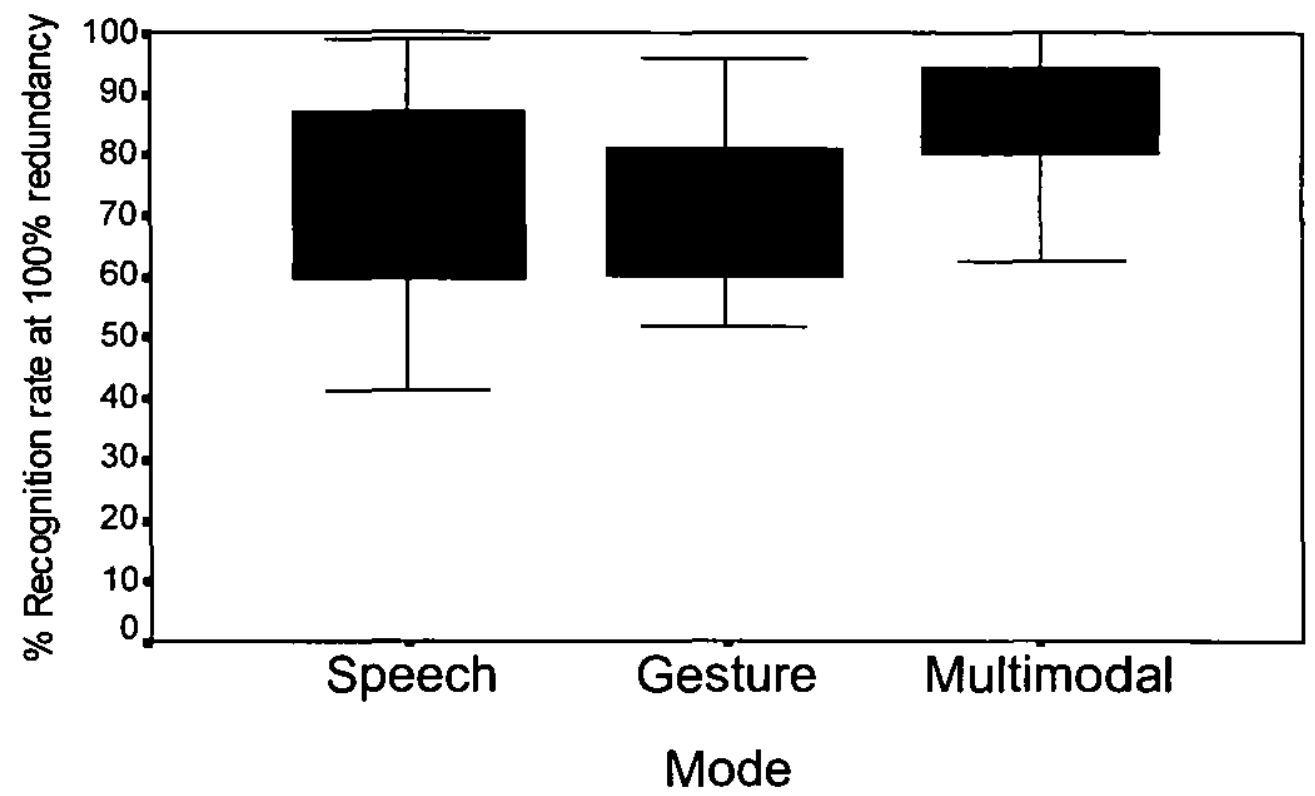

Figure 40: Comparison of speech, gesture and multimodal rates at $100 \%$ redundancy

The mean speech recognition rate is $71.12 \%$. The mean gesture recognition rate is $70.31 \%$ and the mean multimodal recognition rate is $87.43 \%$. This difference between the means is statistically significant at $\mathrm{p}<0.0001(\mathrm{~F}(2,177)=32.05$, $\mathrm{p}<0.0001$ ), and the multimodal recognition rates are therefore statistically significantly higher than the speech and gesture rates. This result shows that when $100 \%$ redundancy exists between the speech and gesture input modes, the multimodal interpretation has improved upon either mode alone. 


\subsubsection{Cut, copy and paste tasks}

The general $100 \%$ redundancy result does not take account of the different task content between cut, copy and paste tasks containing three information fields (or slots, e.g. Cut the Heart from 1) and move tasks containing four information fields (e.g. Move the Earth from 11 to 12). The results for cut, copy and paste tasks alone are shown in Figure 41.

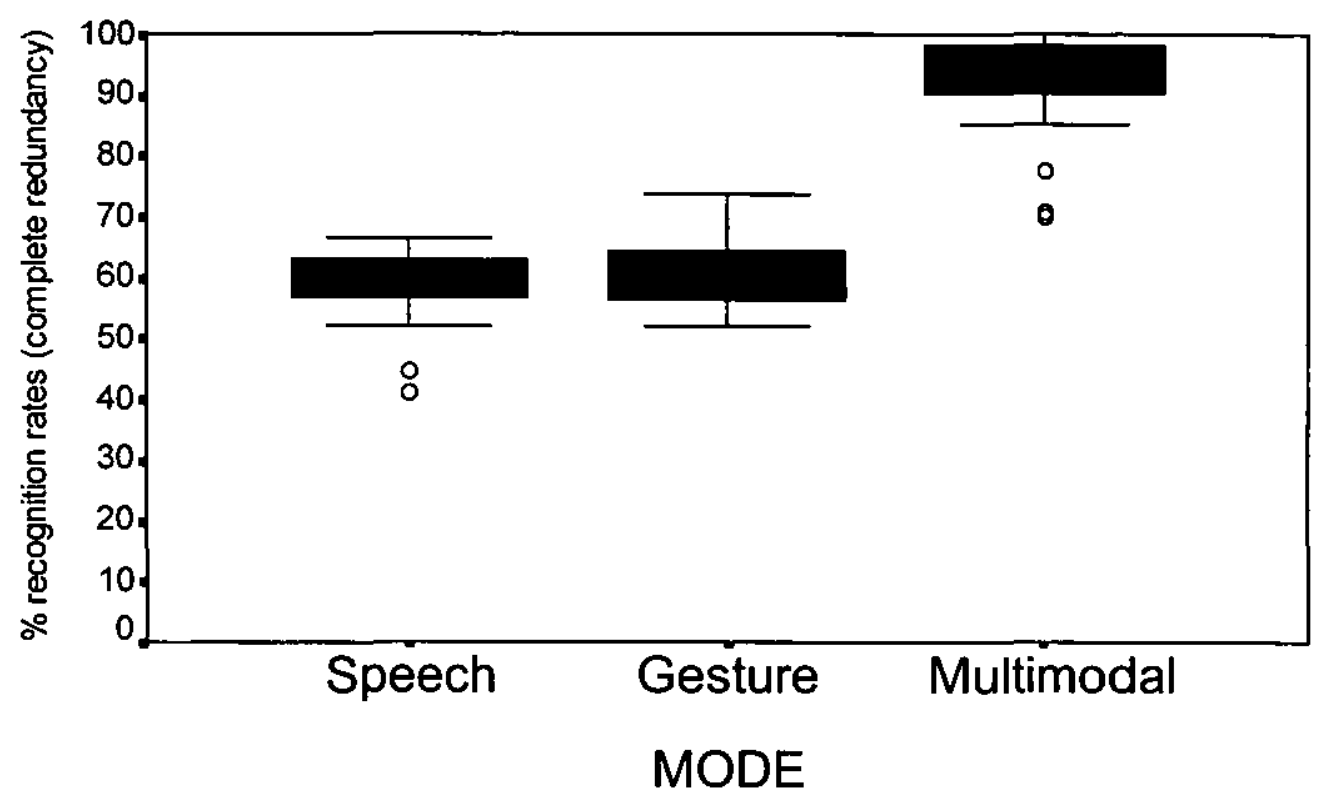

Figure 41: Comparison of speech, gesture and multimodal rates, $100 \%$ redundancy level in cut, copy \& paste tasks

The mean speech recognition rate is $59.26 \%$, and the mean gesture recognition rate is $61.15 \%$. The mean multimodal recognition rate is $92.60 \%$. The multimodal recognition rates are statistically significantly higher than either the speech or gesture rates $(F(2,87)=251.8743, \mathrm{p}<0.0001)$. This results shows that $100 \%$ redundancy between the speech and gesture modes in the cut, copy and paste tasks has given a very high multimodal rate, which is a substantial improvement on either of the unimodal rates. The speech recognition rates represent the success of the system in recognising complete speech tasks including command (cut, copy or paste), object and target fields. The gesture recognition rates have achieved similar levels, showing that recognition of the 
cross, circle and tick gestures (i.e. cut, copy and paste gestures) appears reasonable.

\subsubsection{Move tasks}

The results categorised for move tasks, in complete redundancy cases, is shown in Figure 42.

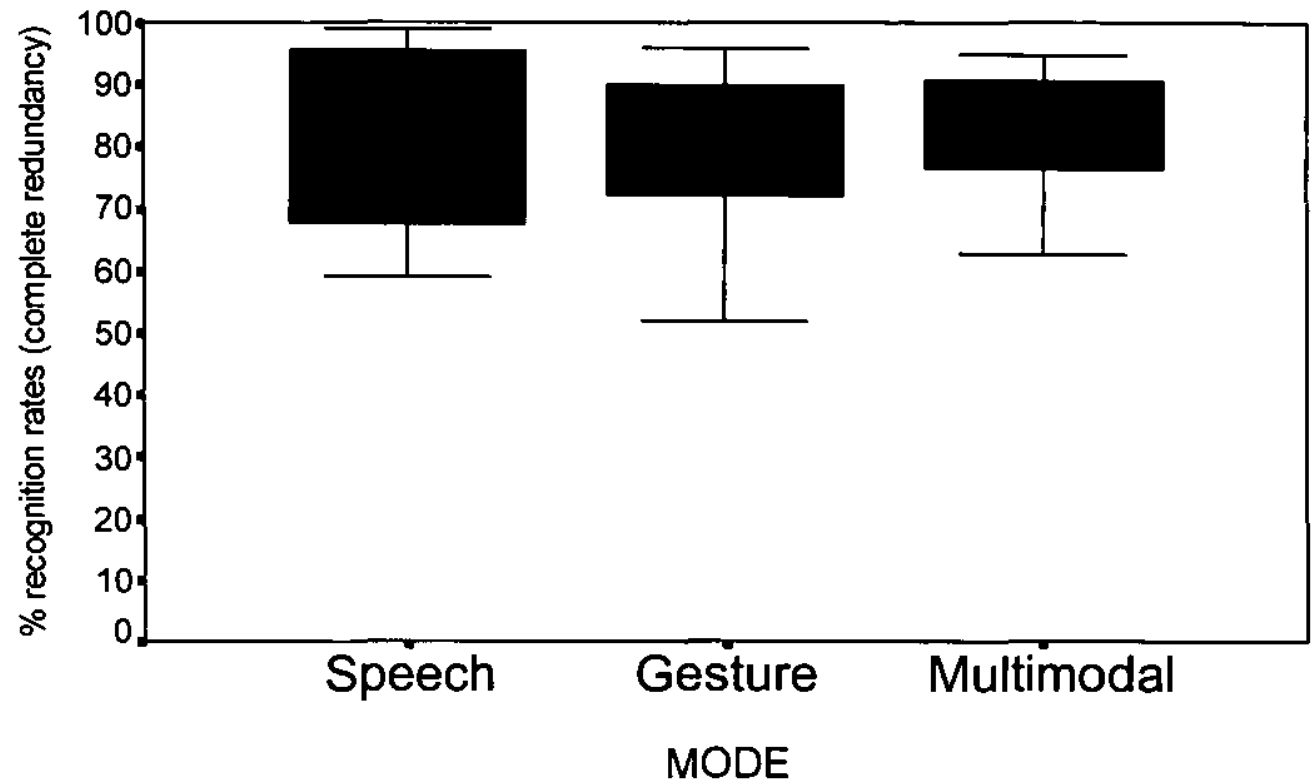

Figure 42: Comparison of speech, gesture and multimodal rates, $100 \%$ redundancy level in move tasks

The mean speech recognition rate is $82.97 \%$ and the mean gesture recognition rate is $79.47 \%$. The mean multimodal recognition rate is $82.25 \%$. There is no statistical difference between any of the means $(F(2,87)=0.7285, p=0.4855)$. This indicates that the presence of redundant information within the move tasks has not created an improvement in the mean multimodal recognition rates, although the spread of the multimodal results is smaller than speech or gesture. This suggests that the combination has created more uniform multimodal recognition rates, indicating that the integration of speech and gesture removes extreme values in the resulting multimodal interpretation. The average speech and gesture recognition rates are also relatively high in this case, and in general 
the rates in the move tasks are higher than in the cut, copy and paste tasks. As both rates are so high, this result may indicate that the multimodal integrator has not been able to improve upon recognition rates at this level. Further analysis will reveal whether this is the case.

Generally, in the complete redundancy case, the presence of redundant information has had more effect in the cut, copy and paste tasks, than in the move tasks The greater complexity involved in the interpretation of the move tasks may explain this result, as these tasks have two number information fields to interpret, compared to one number field in cuts, copies and pastes. It was observed that interpretation of the number input appeared to be poor during experimentation. Further analysis will reveal whether this factor has affected the results.

\subsubsection{Partial redundancy results}

The results in the partial redundancy cases include the $66 \%$ redundancy level (cut, copy and paste tasks) and the $75 \%$ redundancy level (move tasks). The level of redundancy dictates the maximum value which the speech recognition rates can achieve. In the $66 \%$ case, speech recognition rates will be at most $66 \%$ (e.g. the speech input was 'Copy the Heart', the target number field has been omitted). Similarly, speech recognition rates will be at most $75 \%$ in the $75 \%$ redundancy case (e.g. the speech input was 'Move the Ear from 6', the destination field has been omitted). 


\subsubsection{Cut, copy and paste tasks}

The partial redundancy results for cut, copy and paste tasks are shown in Figure 43.

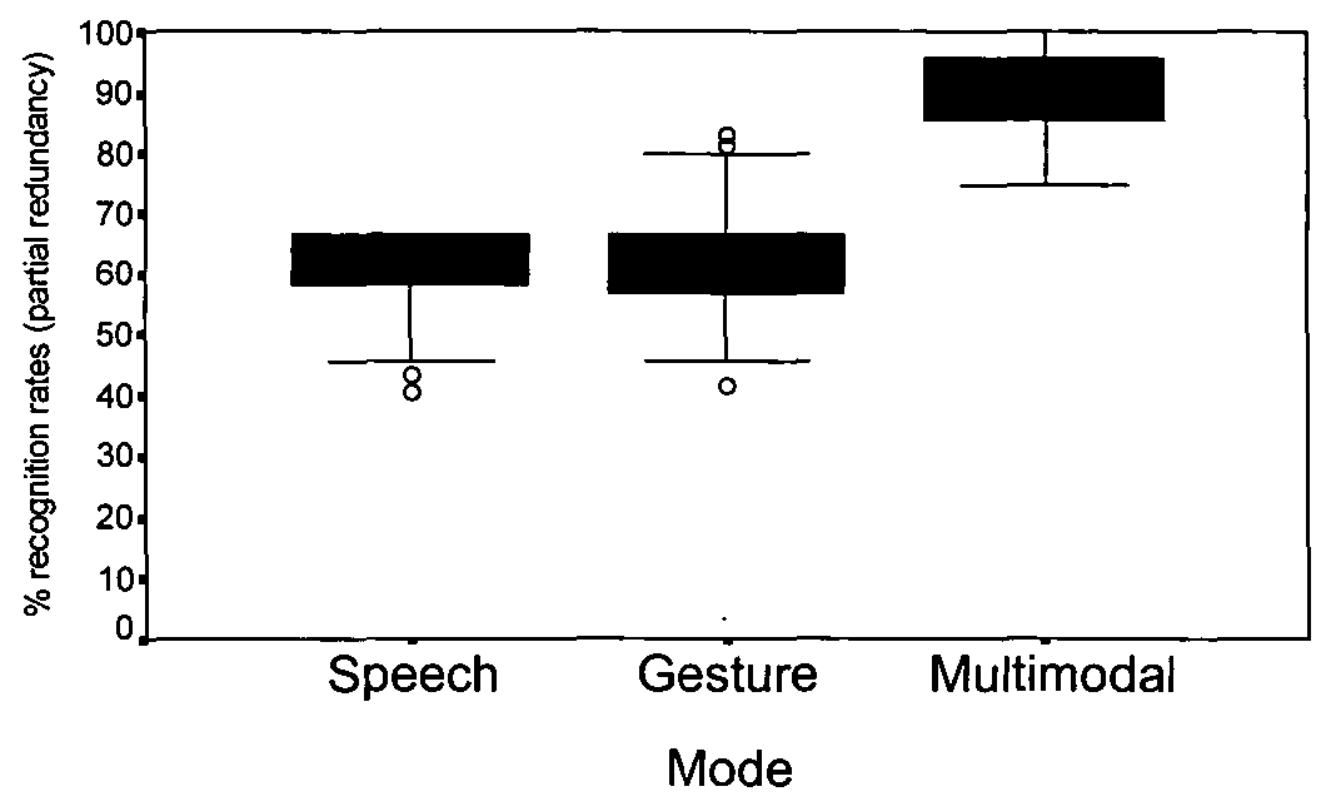

Figure 43: Comparison of speech, gesture and multimodal rates in partial redundancy cases for cut, copy and paste tasks

As we expect the speech recognition rates are lower than the complete redundancy case. The mean speech recognition rate is $60.73 \%$ (the maximum possible is $66.6 \%$ ). The mean gesture recognition rate is $62.28 \%$ and the mean multimodal recognition rate is $89.99 \%$. The multimodal recognition rates are significantly higher statistically than either speech or gesture $(F(2,177)=$ $281.4073, \mathrm{p}<0.0001)$. This result shows that even in the presence of only partial redundancy, the multimodal interpretation has significantly improved upon the unimodal rates alone in cut, copy and paste tasks. 


\subsubsection{Move tasks}

The partial redundancy results for move tasks is shown in Figure 44.

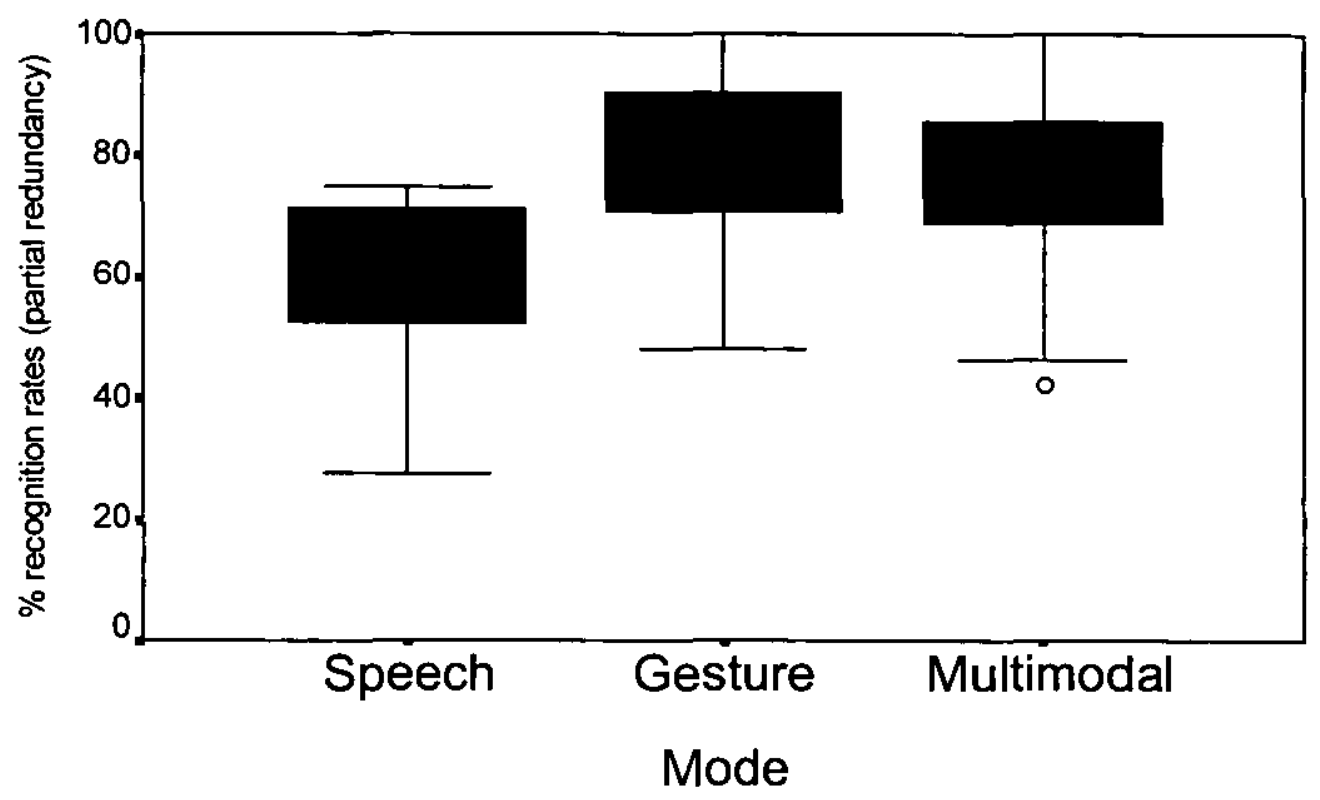

Figure 44: Comparison of speech, gesture, and multimodal recognition rates in partial redundancy cases for move tasks

The mean speech recognition rate is $61.66 \%$ (the maximum possible is $75 \%$ ). The mean gesture recognition rate is $80.30 \%$. The mean multimodal recognition rate is $76.90 \%$. Although the speech recognition results differ significantly from the gesture and multimodal rates, as we expect, there is no significant difference between the gesture and multimodal recognition rates $(F(2,177)=37.7689$, $\mathrm{p}<0.0001)$.

Again the presence of redundant information within move tasks has not provided a significant improvement in the multimodal interpretation. The spread of the multimodal rates is less than either speech or gesture, suggesting that there is some improvement in the results although it is not visible in the mean rates. As in the complete redundancy case, the multimodal rates are more evenly spread, showing that the combination of speech and gesture removes extreme values from the results. 
It is interesting to note that the gesture recognition rates in the partial redundancy move tasks are again relatively high, at around $80 \%$ (recall that the gesture recognition rate was also high in the complete redundancy move tasks at around $80 \%$ ). The corresponding gesture recognition rate for cut, copy and paste tasks is around $60 \%$ in both partial and complete redundancy cases. The possible move gestures are lines (either vertical, horizontal, positive or negative gradient) which the gesture recognition system has more success in recognising when compared to the possible cut, copy and paste gestures of crosses, circles and ticks respectively. This result indicates that the high gesture success in interpreting move gesture tasks is limiting the amount of gain that may be made in a multimodal interpretation.

\subsubsection{Single redundancy results}

The single redundancy results represent the redundancy levels of $33 \%$ for cut, copy and paste tasks, and the $25 \%$ redundancy level for move tasks. Again, the maximum speech rates attainable are governed by the redundancy level: $33 \%$ for cut, copy and paste tasks, and $25 \%$ for move tasks.

\subsubsection{Cut, copy and paste tasks}

The results for the single redundancy level in cut, copy and paste tasks is shown in Figure 45. 


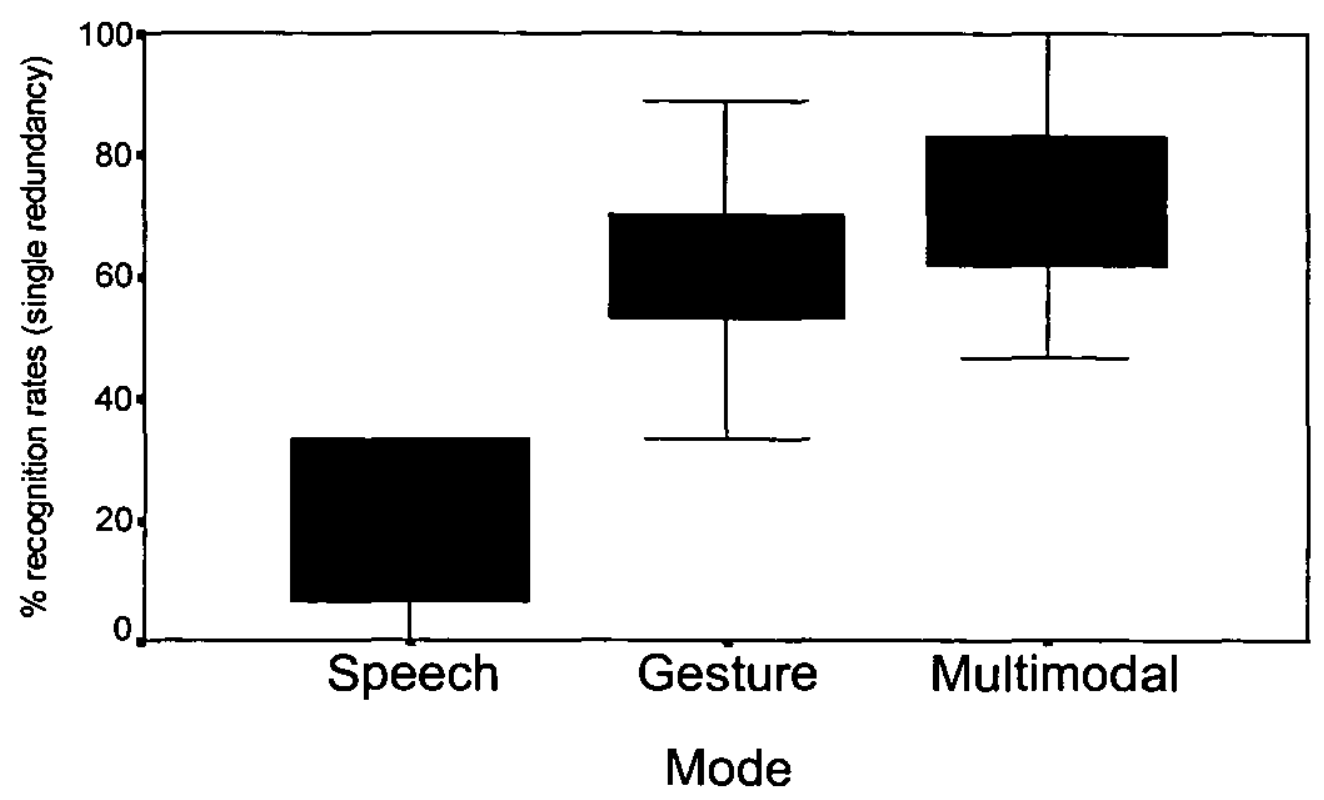

Figure 45: Comparison of speech, gesture and multimodal rates, single redundancy in cut, copy and paste actions

The mean speech recognition rate is $21.44 \%$ (the maximum possible was $33 \%$ ). Again the gesture rates are around $60 \%$, with a mean gesture recognition rate of $61.13 \%$. The mean multimodal recognition rate is $73.65 \%$. The multimodal rates are higher with statistical significance $(\mathrm{F}(2,267)=397.2102, \mathrm{p}<0.0001)$ than either the speech or gesture rates. Even in the presence of limited redundant information, the multimodal interpretation is a significant improvement on the unimodal rates in cut, copy and paste tasks.

\subsubsection{Move tasks}

The single redundancy results are shown in Figure 46. 


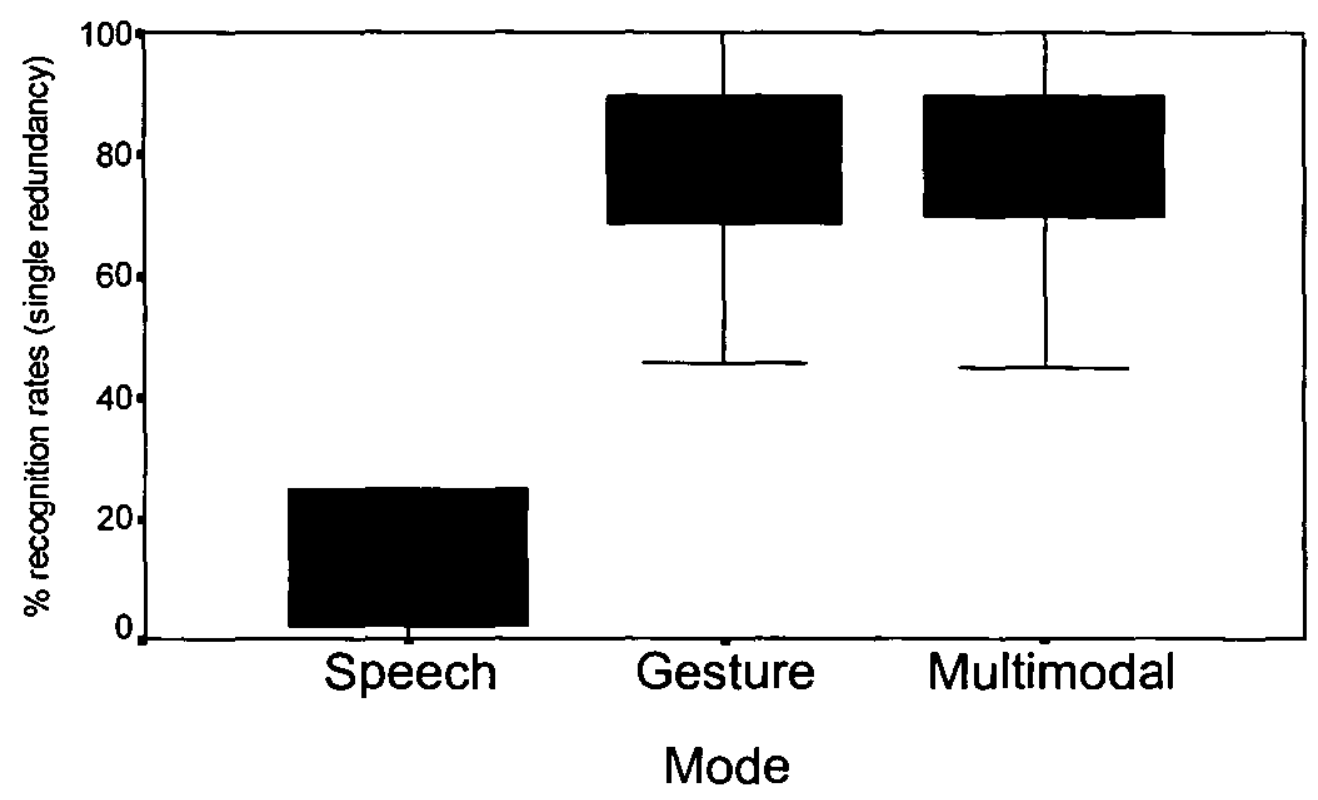

Figure 46: Comparison of speech, gesture and multimodal rates, single redundancy level in move tasks

The mean speech recognition rate is $14.67 \%$ (the maximum possible was $25 \%$ ), and the mean gesture recognition rate is $79.08 \%$. The mean multimodal recognition rate is $79.40 \%$. The difference between gesture and multimodal recognition rates is not significant, although as expected, there is significant differences between the speech and other rates $(F(2,267)=750.9265, p<0.0001)$. Again the results have shown that the presence of redundant information within move tasks has not created a significant improvement in the multimodal interpretation, although the spread of data is slightly smaller in the multimodal case. Additionally the gesture recognition rate for moves has remained around $80 \%$ (the gesture rate in the corresponding cut, copy and paste case was $61 \%$ ). This result shows that in the cases where the gesture recognition success is consistently high, the presence of additional information does little to improve upon an already high rate.

\subsubsection{Discussion}

These initial results are very encouraging. Whilst the interpretation of move tasks has not shown significant improvement with the presence of redundant 
information, the interpretation of cut, copy and paste tasks has been substantially improved. The highest rate achieved in the complete redundancy case is $92.6 \%$. The results also indicate that for move tasks, the gesture recognition rates of around $80 \%$ are high, and significantly higher than in the cut, copy and paste rates of $60 \%$. The multimodal rate has shown little improvement over an already successful recognition rate.

The multimodal recognition rates have been generated with actual, imperfect data from the speech and gesture sources, and in particular with variable speech rates. The imperfect data from the speech and gesture sources included contradictions between the modes, and recognition errors of some or all of the parameters in a phrase. Therefore, even in the presence of possible ambiguities and contradictions, the multimodal interpreter succeeded in correctly combining and recognising input, on average, in $82 \%$ of cases (and as we have seen this rate rises in particular redundancy cases). This compares very favourably with other reported work from implemented multimodal systems.

Vo and Wood, 1996 (Chapter 2) presented a multimodal system (using speech, two-dimensional gesture and handwriting input) which performed recognition at a rate of $80 \%$ in the presence of perfect transcribed input, that is, when the modes did not contain recognition errors. When the actual recognition data from each mode was used, this introduced recognition errors into the system, and the multimodal integrator recognition rate dropped to $35 \%$. This was attributed to the poor performance of the gesture and handwriting recognition system, where some of the heuristics employed had failed, giving rates of around $32 \%$.

\subsection{Analysis of Multimodal Scores and Redundancy}

To explore the effect of the detailed redundancy levels on the final multimodal rates, it was important to investigate the possible relationships between them. To provide a general overview of possible relationships, patterns and effects, an analysis of the sample means of group multimodal performance, according to their redundancy level was carried out. 
Redundancy can be quantified in several ways (Section 7.4). In Table 17, redundancy is expressed as one of five possible values: $25 \%, 33 \%, 66 \%, 75 \%$ or $100 \%$. Several sub-categories within these categories provide further possible groupings. For instance, three types of speech repetition produce $25 \%$ redundancy: repetition of commands, objects and destination slots within move actions. The various sub-categories are shown in Table $17(\mathrm{C}=$ command, $\mathrm{O}=$ object, $\mathrm{T}=$ target, $\mathrm{D}=$ destination).

\begin{tabular}{|c|c|}
\hline Repented Stots & Redondancylevel: \\
\hline $\mathrm{C} 25, \mathrm{O} 25, \mathrm{D} 25$ & $25 \%$ \\
\hline $\mathrm{C} 33, \mathrm{O} 33, \mathrm{~T} 33$ & $33 \%$ \\
\hline CO66, CT66 & $66 \%$ \\
\hline $\mathrm{COD}, \mathrm{COT} 75$ & $75 \%$ \\
\hline COT 100, COTD & $100 \%$ \\
\hline
\end{tabular}

\section{Table 17: Redundancy scores and repeated speech slots}

The sub-categories are labelled according to the repeated slots used and the overall redundancy level they belonged to. For instance, the case CO66 represents the speech requests consisting of command and object combinations in cut, copy and paste tasks. CT66 represents command and target combinations in cut, copy and paste tasks. Where possible ambiguity exists about the overall redundancy level, the level is appended to the combination e.g. COT75 represents command, object and target combinations for move actions ( 3 out of 4 , or $75 \%$ of slots have been repeated). COT100 represents the command, object and target combination for cut, copy and paste tasks.

\subsubsection{Testing for linear correlation}

A test for correlation between multimodal recognition rates and redundancy levels, can be carried out using the five main levels of redundancy. The twelve sub-categories are categorical measures of redundancy, and cannot be used for correlation tests (Moore, 1995). Therefore, a test for correlation between the five redundancy levels of $25 \%, 33 \%, 66 \%, 75 \%$ and $100 \%$ and multimodal scores was performed. This gave $\mathrm{r}=0.2784$ at $\mathrm{p}<0.0001(\mathrm{~N}=360)$. As the sample is large, this is not a significant figure and scatterplots of multimodal scores and redundancy values (Figure 47 ) indicate that the relationship is not linear. The 
result does indicate however, that there is a positive correlation between multimodal recognition rates and the redundancy levels.

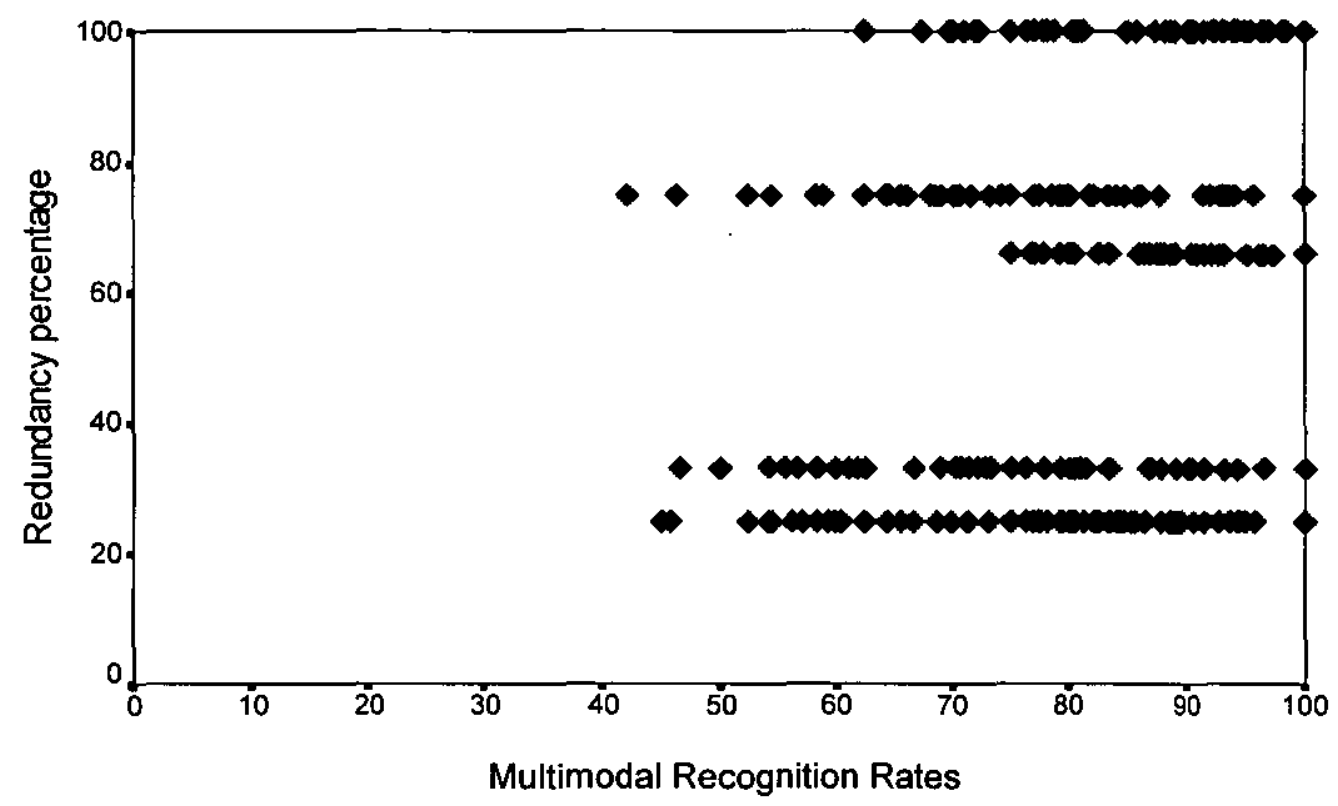

\section{Figure 47: Scatterplot of multimodal recognition rates by redundancy rate}

Further analysis to determine other relationships between redundancy and multimodal scores was therefore required.

To help determine possible relationships for each redundancy level, the data is organised by two main factors:

- $\quad$ Task type, which dictated number of information slots. Cut, copy and paste tasks are analysed together (three slots required for the complete task), and move tasks are analysed separately (four slots required for the complete task).

- Redundancy levels within the tasks. In cut, copy and paste tasks the redundancy levels are 33\% [C33, O33 and T33], 66\% [CO66 and CT66], and $100 \%$ [COT]. In move tasks the redundancy levels are $25 \%$ [C25, O25, and D25], 75\% [COD and COT75] and 100\% [COTD].

This approach will be used for the analysis which follows. 


\subsubsection{Investigating relationships between redundancy and recognition rates}

\subsubsection{Five main redundancy levels}

The speech, gesture and multimodal recognition rates, for cut, copy and paste actions, are shown in Figure 48. These graphs are grouping the results from Section 8.5 together for comparison of the rates per redundancy level. The results for move actions are shown in Figure 49.

The lines at $33 \%$ and $66 \%$ in Figure 48 indicate the maximum possible values that these speech recognition rates could achieve in the $33 \%$ and $66 \%$ redundancy cases respectively (as the speech input had partial task content e.g. 'Cut', 'Copy the Flower'). Recall that the circles plotted are outliers which represent values that fall outside the general spread of the data.

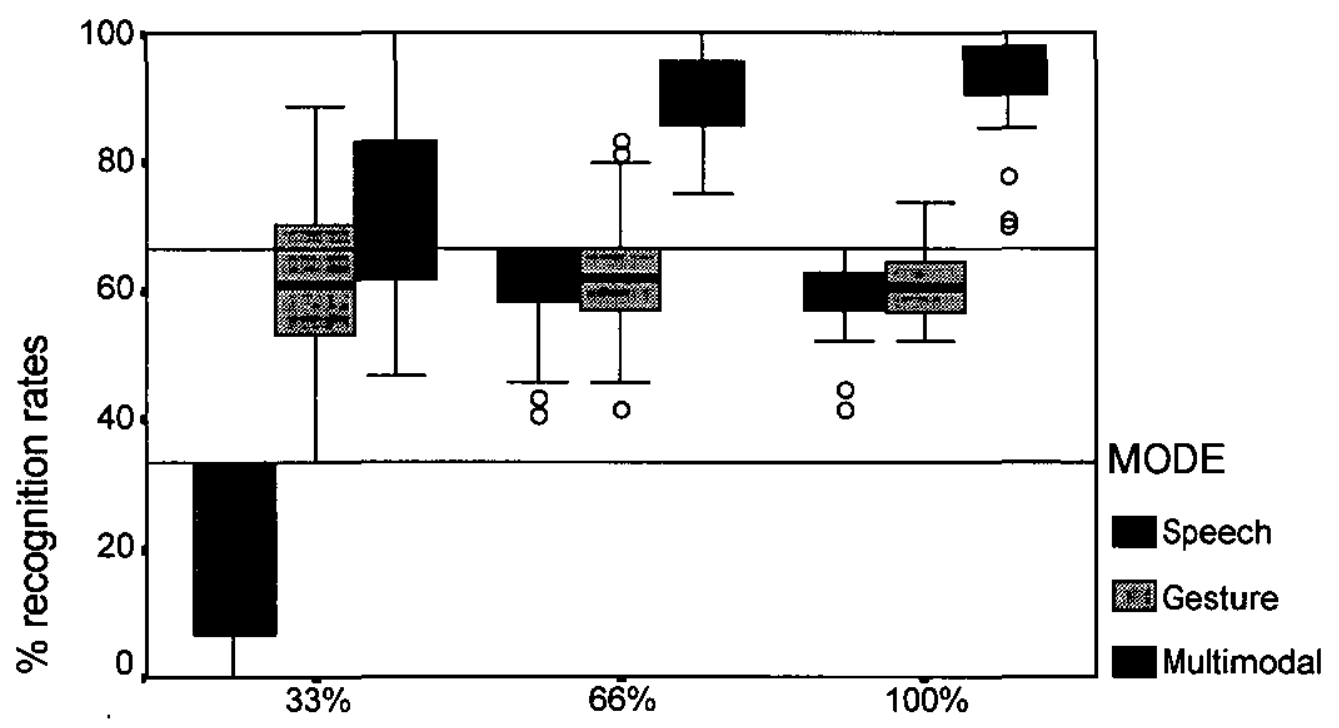

Redundancy level

Figure 48: Boxplot of average speech, gesture and multimodal rates for cut, copy and paste actions

These general results show that there is a high degree of variability in the speech rates in the $33 \%$ case, while the $66 \%$ and $100 \%$ rates show less variability. The multimodal recognition rates show a general upward trend as redundancy levels rise, which is encouraging,-and these rates are also higher than the gesture rates in- 
each case. Analysis of the sub-categories of redundancy may reveal more about the recognition rates, and whether the presence of different information had an effect on rates (e.g. words versus numbers).

Figure 49 shows the results for the move actions. The lines at $25 \%$ and $75 \%$ indicate the maximum possible speech rates for the $25 \%$ and $75 \%$ redundancy cases respectively (as the speech input contained partial task content e.g. 'Move', 'Move the Heart to 11', 'Move the Flowerpot from 23').

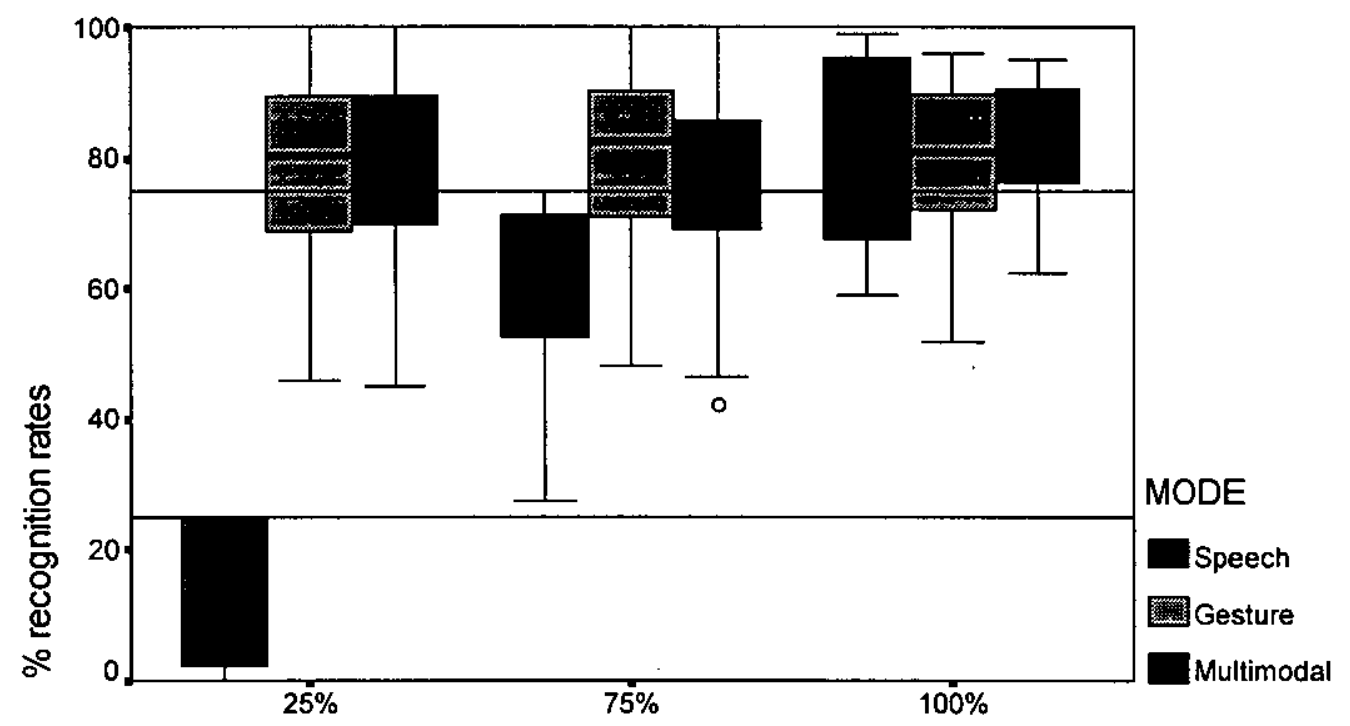

Redundancy level

Figure 49: Boxplot of average speech, gesture and multimodal scores for move actions

The general results for the move actions show that, again, there is high variability in the low redundancy speech cases $(25 \%)$. Multimodal recognition rates are higher in the $25 \%$ redundancy cases, but marginally lower in the $75 \%$ and $100 \%$ cases. There is no indication of an increase in multimodal recognition rates as the redundancy levels rise. Analysis of the twelve sub-categories is therefore also required for move actions.

The analysis over the five redundancy levels and all task types shows that multimodal recognition rates are generally distributed over a smaller range than 
either speech or gesture rates. This indicates that the multimodal recognition rates are more invariant and stable than the unimodal rates. In the simpler cut, copy and paste tasks, the multimodal recognition rates are higher in all redundancy cases, while the more complex move tasks have shown less success in recognition rates.

\subsubsection{Twelve redundancy levels}

Cut, copy and paste tasks

The speech, gesture and multimodal recognition rates for cut, copy and paste actions, across the redundancy sub-categories, are shown in Figure 50. The lines drawn at $33 \%$ and $66 \%$ indicate the maximum possible rates that speech recognition can achieve in the $33 \%$ and $66 \%$ redundancy cases.

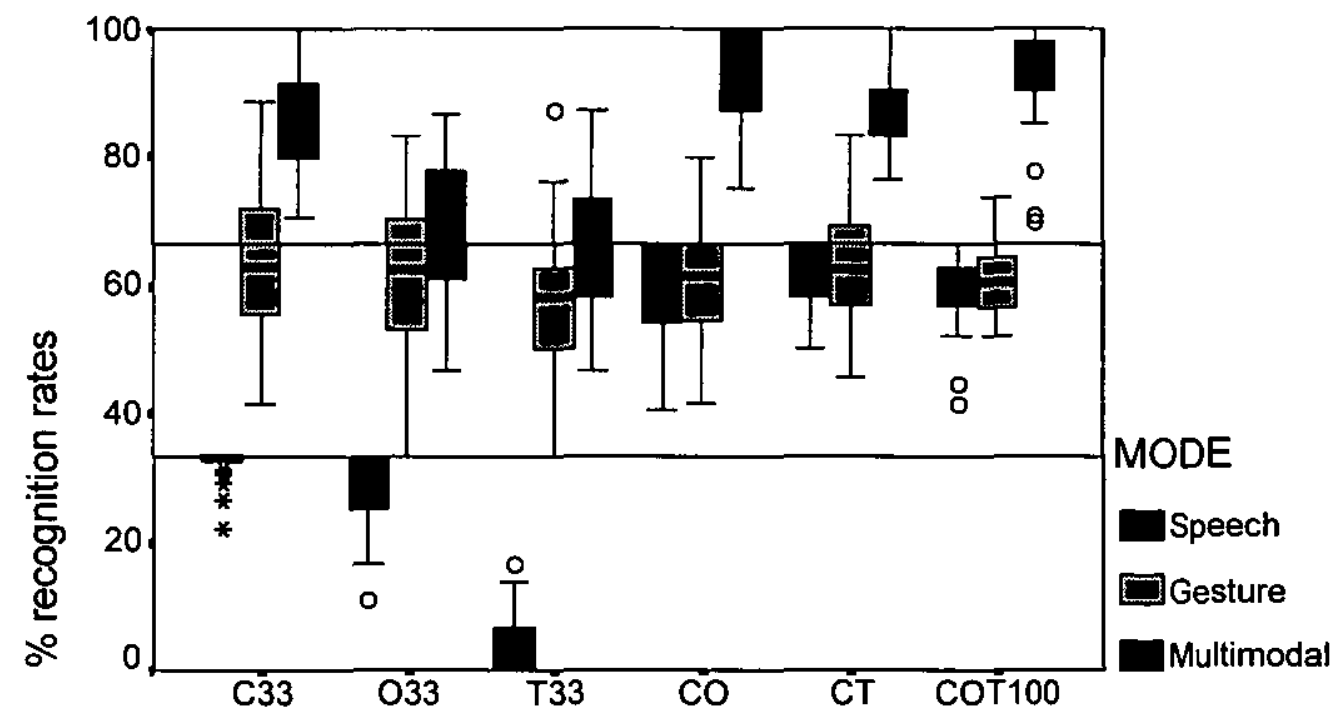

Redundancy level

Figure 50: Speech, gesture and multimodal recognition rates for cut. copy and paste tasks

The results for cut, copy and paste actions indicate the following:

$33 \%$ case - the multimodal recognition rates are highest in the presence of redundant command information, slightly lower in the presence of redundant 
object information and lowest in the presence of redundant target number information. Multimodal recognition rates are higher than gesture recognition rates, although in a similar range in the 033 and T33 cases.

- C33 - speech recognition rates in this case are very high (lying on the maximum line). This means that the speech recognition of the words cut, copy and paste was very successful. Multimodal rates are higher than gesture recognition rates, indicating that the extra information from speech has improved the overall task interpretation. The presence of extra command information had generated significant improvements.

- $\quad 033$ - the presence of redundant object information has also generated substantial improvements in the multimodal rates, although less significantly than the command case.

- T33 - speech recognition of the target numbers is very poor. However, the multimodal recognition rates are higher than the gesture rates, indicating that even a small improvement can be generated with the presence of some redundant information.

$66 \%$ case - the multimodal recognition rates are substantially higher than gesture recognition rates in both $66 \%$ cases. The speech and gesture recognition rates have achieved similar ranges of recognition success.

- $\quad$ CO66 - In the presence of redundant command and object information, the speech and gesture rates are similar. Multimodal recognition rates are substantially higher than gesture rates, and are the highest out of this task set (cut, copy and paste tasks). The presence of pivotal redundant information has had a significant effect on the multimodal interpretation.

- CT66 - Multimodal recognition rates are substantially higher than gesture rates, although the gain is lower than in the CO66 case. It is suspected that the multimodal interpretation of the target numbers is less successful than the command and object interpretation (as displayed in the C33 and T33 results). Further analysis will be required to confirm this suspicion. This explains why the multimodal rates of the CT66 case are lower than those of the CO66 case.

$100 \%$ case

- COT100 - Speech and gesture rates are at similar levels, slightly lower than the other cases. The multimodal recognition rates are substantially higher than speech and gesture, although slightly lower than the multimodal rate achieved in the CO66 case. Again, this can be attributed to the poor interpretation of the target numbers, which may have acted to lower the overall multimodal rates.

The descriptive statistics for these results are shown in Table 18. 


\begin{tabular}{llllll}
\hline Redundancy level & & Mean & Std Deviation & Maximum & Minimum \\
\hline C33 & Speech & 32.16 & 2.57 & 33.33 & 22.22 \\
& Gesture & 64.48 & 12.17 & 88.89 & 41.67 \\
& Multimodal & 86.35 & 8.50 & 100.00 & 70.83 \\
\hline O33 & Speech & 28.07 & 5.81 & 33.33 & 11.11 \\
& Gesture & 60.87 & 12.78 & 83.33 & 33.33 \\
& Multimodal & 70.06 & 10.67 & 86.67 & 46.67 \\
\hline T33 & Speech & 4.10 & 4.48 & 16.67 & .00 \\
& Gesture & 58.03 & 11.18 & 87.50 & 33.33 \\
& Multimodal & 64.54 & 10.19 & 87.50 & 46.67 \\
\hline CO66 & Speech & 59.57 & 7.78 & 66.67 & 40.74 \\
& Gesture & 61.26 & 8.93 & 80.00 & 41.67 \\
& Multimodal & 93.00 & 7.29 & 100.00 & 75.00 \\
\hline CT66 & Speech & 61.90 & 4.63 & 66.67 & 50.00 \\
& Gesture & 63.30 & 9.15 & 83.33 & 45.83 \\
& Multimodal & 86.98 & 5.52 & 100.00 & 76.67 \\
\hline COT100 & Speech & 59.26 & 5.78 & 66.67 & 41.48 \\
& Gesture & 61.15 & 5.66 & 73.81 & 52.17 \\
& Multimodal & 92.60 & 7.73 & 100.00 & 70.10 \\
\hline
\end{tabular}

Table 18: Descriptive statistics of speech, gesture and multimodal rates in cut, copy and paste tasks

These results show that the multimodal recognition rates are higher than the gesture rates in each of the redundancy levels. The multimodal recognition rates are substantially higher in each category, suggesting that the presence of extra information has created a multimodal rate which is an improvement on the gesture rate.

The CO66 redundancy level gives the highest multimodal recognition rates, and the COT100 level also generates high multimodal rates. The presence of redundant number information had not produced as substantial an effect as the other cases, where non-number task information (command and object information) is present.

In the cases where substantial redundant information is present, and speech and gesture recognition rates are comparable, the multimodal recognition rates are a substantial improvement upon the either of unimodal recognition rates alone. Multimodal recognition rates have risen as the amount of redundancy between modes is increased. 
Move tasks

The results for move actions are shown in Figure 51. The lines at $25 \%$ and $75 \%$ indicate the maximum possible speech rates in the $25 \%$ and $75 \%$ redundancy cases.

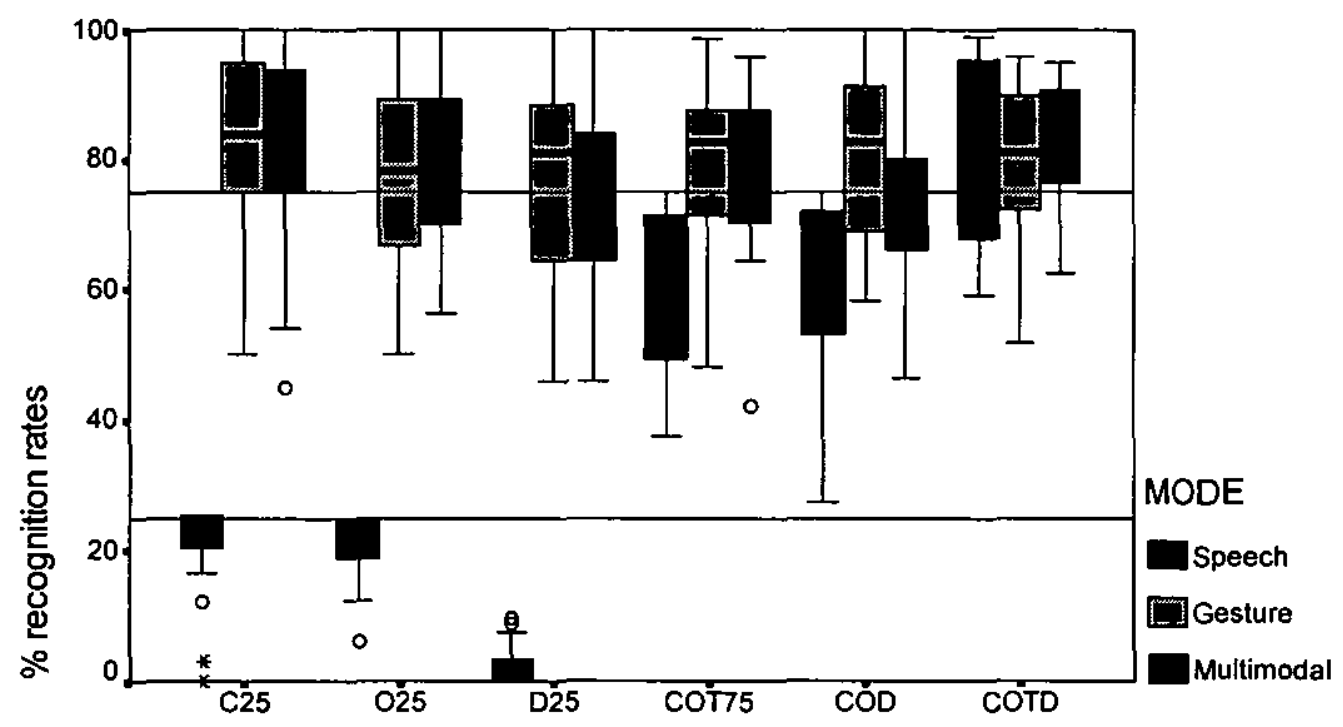

Redundancy level

Figure 51: Speech, gesture and multimodal recognition rates for move tasks 
The results for the move tasks indicate the following:

$25 \%$ case - multimodal recognition rates have mostly improved upon the gesture rates, although the destination number case is an exception.

- $\quad \mathrm{C} 25$ - multimodal recognition rates are marginally higher than the gesture recognition rates. The speech input has been successfully recognised, with recognition rates of around the maximum $25 \%$. The presence of this redundant command information has created a small improvement in the multimodal recognition rates over the gesture recognition rates.

- $\quad$ O25 - Similarly, the speech recognition rates of the object information are good, around $25 \%$. This redundant object information has again created multimodal recognition rates which are higher than the gesture rates. Both the gesture and multimodal recognition rates are lower in the object case than the command case.

- D25 - The speech recognition and interpretation of destination numbers is very low. The multimodal recognition rates have not improved upon the gesture recognition rates in this case.

$\mathbf{7 5 \%}$ case - The multimodal recognition rates are lower than the gesture rates in both cases, although speech recognition rates are reasonably high (average of around $60 \%$ in both cases)

- $\quad$ COT75 - The multimodal recognition rates, which are lower than the gesture rates, do not appear significantly lower.

- $\quad$ COD - The multimodal recognition rates appear substantially lower than the gesture rates, and both rates are lower than in the COT case.

\section{$100 \%$ case}

- COTD - multimodal recognition rates are marginally higher than gesture rates, while speech rates are high.

The descriptive statistics for the speech, gesture and multimodal rates over the sub-categories of redundancy levels are shown in Table 19. 


\begin{tabular}{llllll}
\hline Redundancy level & & Mean & Std Deviation & Maximum & Minimum \\
\hline C25 & Speech & 20.62 & 8.43 & 25.00 & .00 \\
& Gesture & 82.90 & 14.08 & 100.00 & 50.00 \\
O25 & Multimodal & 83.09 & 14.12 & 100.00 & 45.00 \\
& Speech & 21.10 & 4.53 & 25.00 & 6.25 \\
& Gesture & 77.23 & 13.73 & 100.00 & 50.00 \\
D25 & Multimodal & 78.94 & 12.30 & 100.00 & 56.25 \\
& Speech & 2.27 & 3.01 & 10.00 & .00 \\
& Gesture & 77.10 & 14.70 & 100.00 & 45.83 \\
COT75 & Multimodal & 76.16 & 13.91 & 100.00 & 45.83 \\
& Speech & 62.95 & 11.66 & 75.00 & 37.50 \\
& Gesture & 79.53 & 12.39 & 98.68 & 48.08 \\
COD & Multimodal & 78.90 & 12.22 & 95.83 & 42.31 \\
& Speech & 60.37 & 13.15 & 75.00 & 27.50 \\
& Gesture & 81.07 & 12.70 & 100.00 & 58.33 \\
COTD & Multimodal & 74.90 & 13.02 & 100.00 & 46.43 \\
& Speech & 82.97 & 13.62 & 99.00 & 59.09 \\
& Gesture & 79.47 & 11.98 & 96.00 & 51.79 \\
& Multimodal & 82.25 & 9.55 & 95.00 & 62.50 \\
\hline
\end{tabular}

Table 19: Descriptive statistics of speech, gesture and multimodal rates in move tasks

By inspection of the descriptive statistics and the boxplots, multimodal recognition rates are more variable in the move tasks. Multimodal recognition rates are higher than gesture recognition rates in the $\mathrm{C} 25$ and $\mathrm{O} 25$ redundancy cases, and marginally lower in the COTD case. In the other cases the multimodal recognition rates are lower than the gesture rates.

Overall, these results show that the more complex move tasks, where the complete interpretation is dependent on the correct recognition and interpretation of target and destination numbers, presented more difficulty to the multimodal integrator. It was observed that overall interpretation of target and destination numbers was poor during experimentation. Statistical analysis to confirm these observations is required. Analysis also helps to identify optimum redundancy levels.

\subsubsection{Confirmation of Higher Multimodal Rates}

To test the hypothesis that various levels of redundancy has an effect on the multimodal rates produced, a series of ANOVAs has been performed. The speech, gesture and multimodal recognition rates are analysed for significant differences, grouped by the distinct levels of redundancy, and organised by task type. The results are for cut, copy and paste tasks are shown in Table 20. 
Non-parametric one-way ANOVAs (Kruskal-Wallis one-way ANOVA) are used since the samples have uniform or non-normal distributions. The ANOVA results are shown as $\chi^{2}$ figures in the tables, with associated $\mathrm{p}$ values indicating the significance of difference (if any) between speech, gesture and multimodal. The statistically significant highest mode is indicated where appropriate.

\begin{tabular}{lll}
\hline $\begin{array}{l}\text { Redundancy } \\
\text { level }\end{array}$ & Mean Ranks & $\begin{array}{l}\text { ANOVA result \& } \\
\text { Significance level }\end{array}$ \\
\hline C33 & speech $=15.50$, gesture $=47.63$, multimodal $=73.37$ & $\chi^{2}=75.18, p<0.0001$ \\
O33 & speech $=15.70$, gesture $=54.00$, multimodal $=66.80$ & $\chi^{2}=62.47, p<0.0001$ \\
T33 & speech $=15.50$, gesture $=55.55$, multimodal $=65.45$ & $\chi^{2}=61.74, p<0.0001$ \\
CO66 & speech $=29.50$, gesture $=31.60$, multimodal $=75.40$ & $\chi^{2}=59.35, p<0.0001$ \\
CT66 & speech $=29.75$, gesture $=31.72$, multimodal $=75.03$ & $\chi^{2}=57.82, p<0.0001$ \\
COT100 & speech $=29.25$, gesture $=31.88$, multimodal $=75.37$ & $\chi^{2}=58.99, p<0.0001$ \\
\hline
\end{tabular}

Table 20: Results of ANOVA for cut, copy and paste tasks

The results show that the multimodal recognition rates are significantly higher than the gesture rates for all of the distinct redundancy levels. Thus, redundancy affects the multimodal recognition rates.

All redundancy levels have produced multimodal recognition rates which are higher than the gesture recognition rates, and obviously higher than the speech recognition rates. The CO66 and COT100 cases appear to have generated the highest multimodal recognition rates (shown in the comparison of the mean rank scores). The redundancy levels containing redundant command information have given the statistically significantly highest multimodal results.

The ANOVA results for the move tasks are shown in Table 21. 


\begin{tabular}{lll}
\hline $\begin{array}{l}\text { Redundancy } \\
\text { level }\end{array}$ & Mean Ranks & $\begin{array}{l}\text { ANOVA result \& } \\
\text { Significance level }\end{array}$ \\
\hline C25 & speech $=15.50$, gesture $=60.37$, multimodal $=60.63$ & $\chi^{2}=60.13, \mathrm{p}<0.0001$ \\
O25 & speech $=15.50$, gesture $=59.40$, multimodal $=61.60$ & $\chi^{2}=59.58, \mathrm{p}<0.0001$ \\
D25 & speech $=15.50$, gesture $=61.68$, multimodal $=59.32$ & $\chi^{2}=59.75, \mathrm{p}<0.0001$ \\
COT75 & speech $=25.93$, gesture $=56.30$, multimodal $=54.27$ & $\chi^{2}=25.34, p<0.0001$ \\
COD & speech $=25.33$, gesture $=60.70$, multimodal $=50.47$ & $\chi^{2}=29.17, p<0.0001$ \\
COTD & speech $=50.32$, gesture $=40.43$, multimodal $=45.75$ & $\chi^{2}=2.15, p=0.3410$ \\
\hline
\end{tabular}

\section{Table 21: Results of ANOVA for move tasks}

The ANOVA results for the move tasks show that there are no significant differences between the gesture and multimodal rates in any of the redundancy levels. As expected there are differences between the speech rates and the other rates. Although the multimodal recognition rates are marginally higher in some of the move redundancy levels, these differences are not significant. This is attributed to the complexity of interpreting between target and destination numbers in the move tasks. This complication was not a factor in the interpretation of cut, copy and paste tasks which contained only one number field. Another factor is the very high gesture rates which the multimodal rates have had difficulty improving upon. The presence of redundant speech information has had little effect on an already high gesture recognition rate of approximately $80 \%$ in each redundancy level.

The results show that there are significant differences between the effect of redundancy in simple versus more complex tasks. Multimodal recognition rates are higher in the cut, copy and paste tasks, which have at most three information slots to complete, compared to the four slots of move tasks.

\subsubsection{Optimum Redundancy level}

To discover whether an optimum multimodal rate is produced from any particular redundancy level, a non-parametric ANOVA was performed on the average multimodal rates by redundancy levels. The results are shown in Table 22 . 


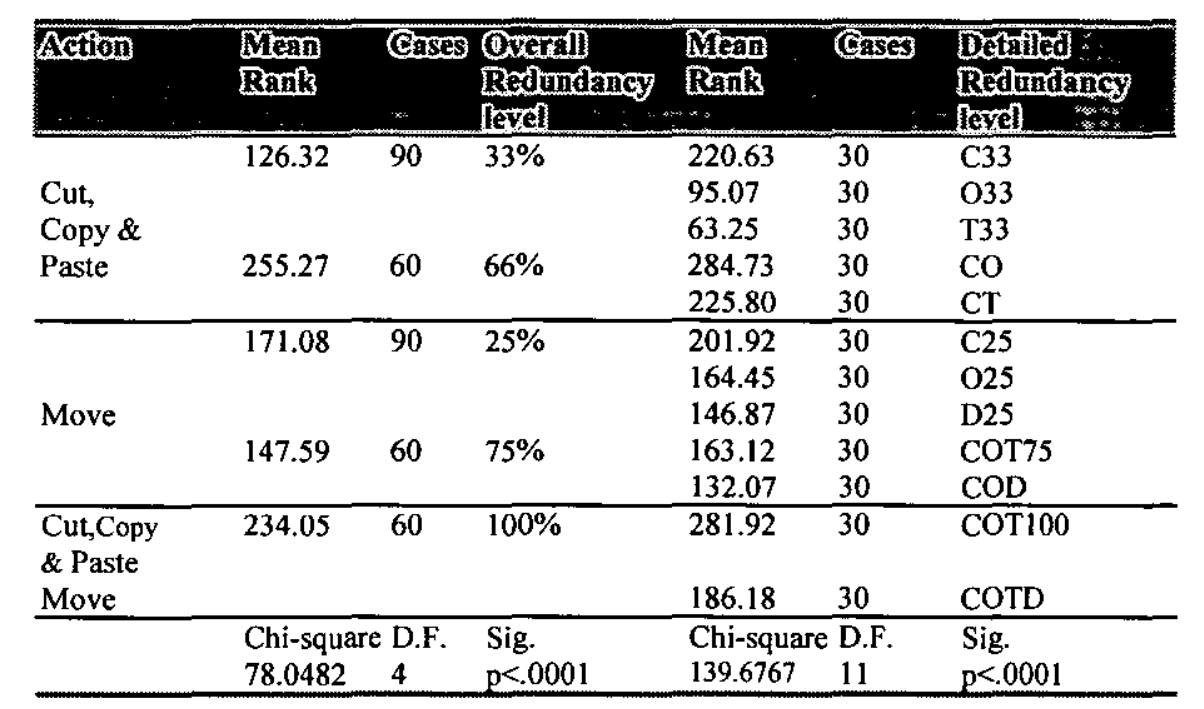

Table 22: Kruskal-Wallis ANOVA results for multimodal scores by five and twelve redundancy levels

The results of the ANOVAs in Table 22 indicate that there were significant differences between the groups.

The mean ranks show that a redundancy level of $66 \%$ gives the significantly highest multimodal score, closely followed by the $100 \%$ redundancy level. This is reflected in the detailed redundancy level where the CO66 redundancy slot (command and object slots were repeated in cut, copy and paste actions) gives the highest multimodal scores, again closely followed by the COT100 slot (command, object plus target slots repeated in cut, copy and paste actions).

Therefore, these preliminary results, while indicating that redundancy of information has a bearing on improving the input recognition task, also show that more complex requests (i.e. the move actions) have presented particular recognition difficulties to the system. The optimum redundancy levels apply to the cut, copy and paste actions, indicating that these actions, which are simpler in structure than move actions, are processed more successfully by the integrator. The actual redundancy levels identified as generating the best multimodal recognition rates are consistent with expectations. The cases of $66 \%$ and $100 \%$ redundancy produce the highest multimodal recognition rates, therefore showing that the exploitation of as much redundant information as possible in the system, does provide significant recognition benefits. 
The results also show that the repetition of the command slot, provides significant improvements in the multimodal recognition rates. The presence of extra information to enable interpretation of the command part of actions contributes significantly to the recognition rates. Those actions where redundant command information is not available show significantly lower multimodal recognition rates (for example, the cases O33, T33, O25, and D25 all have a mean rank of less than 200 in Table 22). Also, on average the mean ranks of the multimodal scores for the move actions are also less than 200 in Table 22. This indicates that the move actions were processed less successfully by the multimodal integrator, because of the difficulty in resolving target and destination references.

\subsection{Further analysis}

Further analysis of the data was performed. In particular the recognition rates of the individual slot types is examined.

\subsubsection{Comparison of primitives recognition over modes}

Another method of analysing the figures gained from the experiments included measuring the overall percentage of successfully recognised cases for each main 'primitive' present in the task requests. These primitives were the command, object, target and destination components of each task. This analysis can confirm if the general multimodal interpretation of target and destination numbers is low, as expected.

The complete vocabulary applying to speech tasks consisted of four command primitives [cut, copy, paste, move] and 7 object primitives [heart, flower, fire, ear, earth, flowerpot, tick] as well as 25 possible target and destination grid numbers [ 1 to 25$]$.

The complete vocabulary applying to gesture tasks consisted of seven command primitives [cut, copy, paste, move vertical, move horizontal, move negative, move positive] , and 7 implied object primitives [heart, flower, fire, ear, earth, flowerpot, 
tick] (objects are implied as the object information can always be deducted when a gesture is performed on a grid location). There are also 25 possible target and destination grid numbers [1 to 25]. For multimodal slots, the primitives were regarded as being the equivalent of the gesture primitives, of which the speech primitives were simply a subset.

For each subject, the number of cases where the first choice of the recognised speech, gesture and multimodal slots matched the requested slot was counted, and divided by the total number of slots that had been requested. This provided summary scores across the complete experiment. Gesture and multimodal rates could then be compared to determine whether generally, multimodal interpretations had improved upon the gesture figures. As in previous sections, the speech recognition figures are not used in the comparison, since they are calculated over a different number of total slots.

Figure 52 shows the speech results for command, object, target and destination slots. These figures show the relative performance of the speech recognition on the individual slots. Figure 53 shows the results for gesture recognition and Figure 54 shows the multimodal recognition results (Table 25 and Table 26, Appendix A, for recognition data). The object gesture recognition rates are dependent on the target and destination gesture recognition rates, since interpretation of the object was dependent on the interpretation of the location information. The object rates shown in Figure 53 are therefore a function of the target and destination results in that figure. As in previous sections, average speech recognition rates are not calculated as task content differed between the speech and other (gesture and multimodal) rates. 


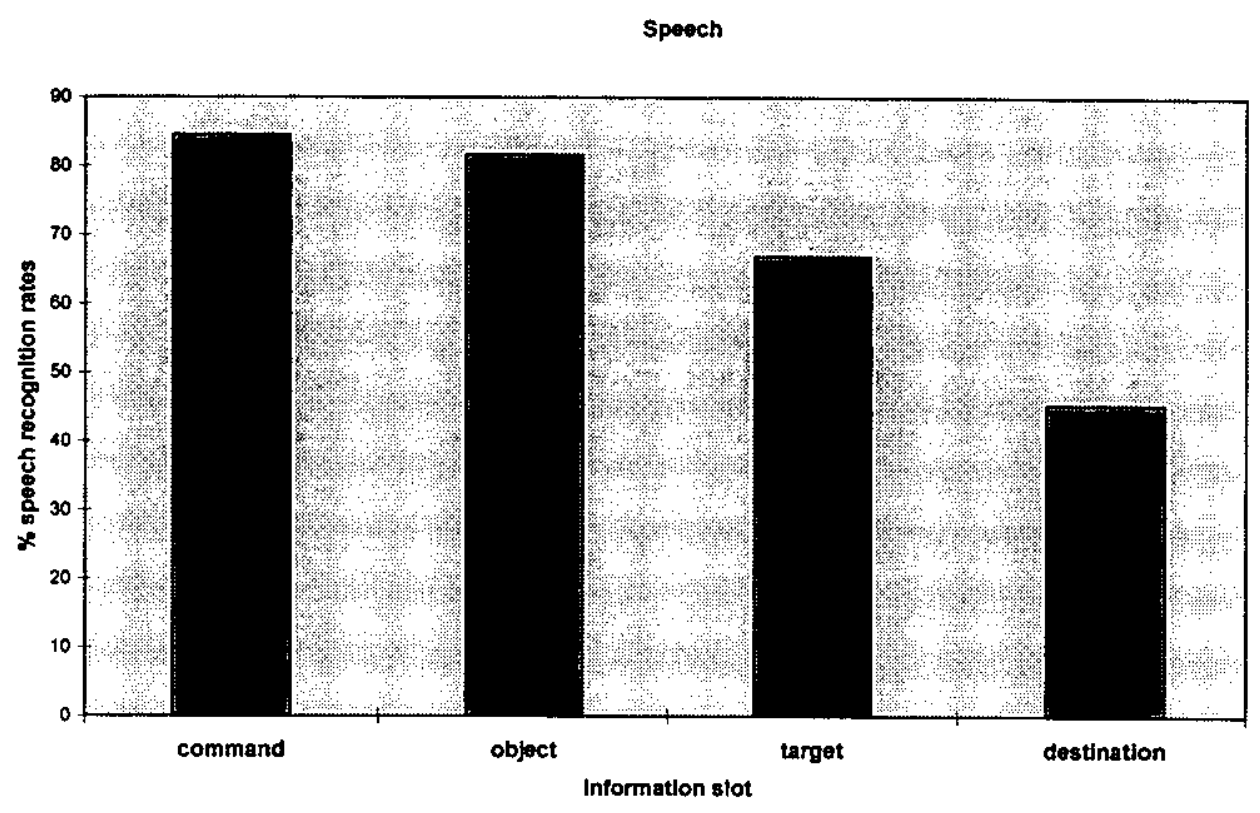

Figure 52: Average first choice speech recognition results for command, object, target and destination types

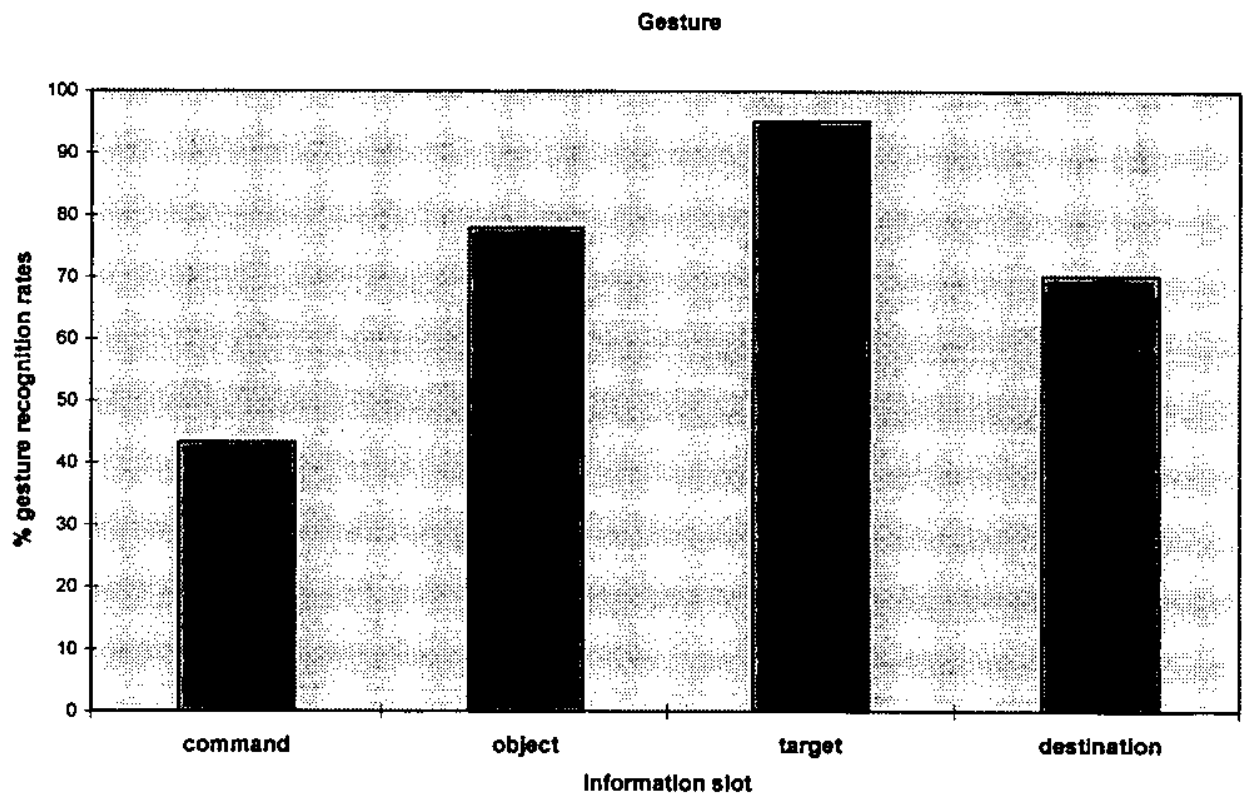

Figure 53: Average first choice gesture recognition results for command, object, target and destination types 


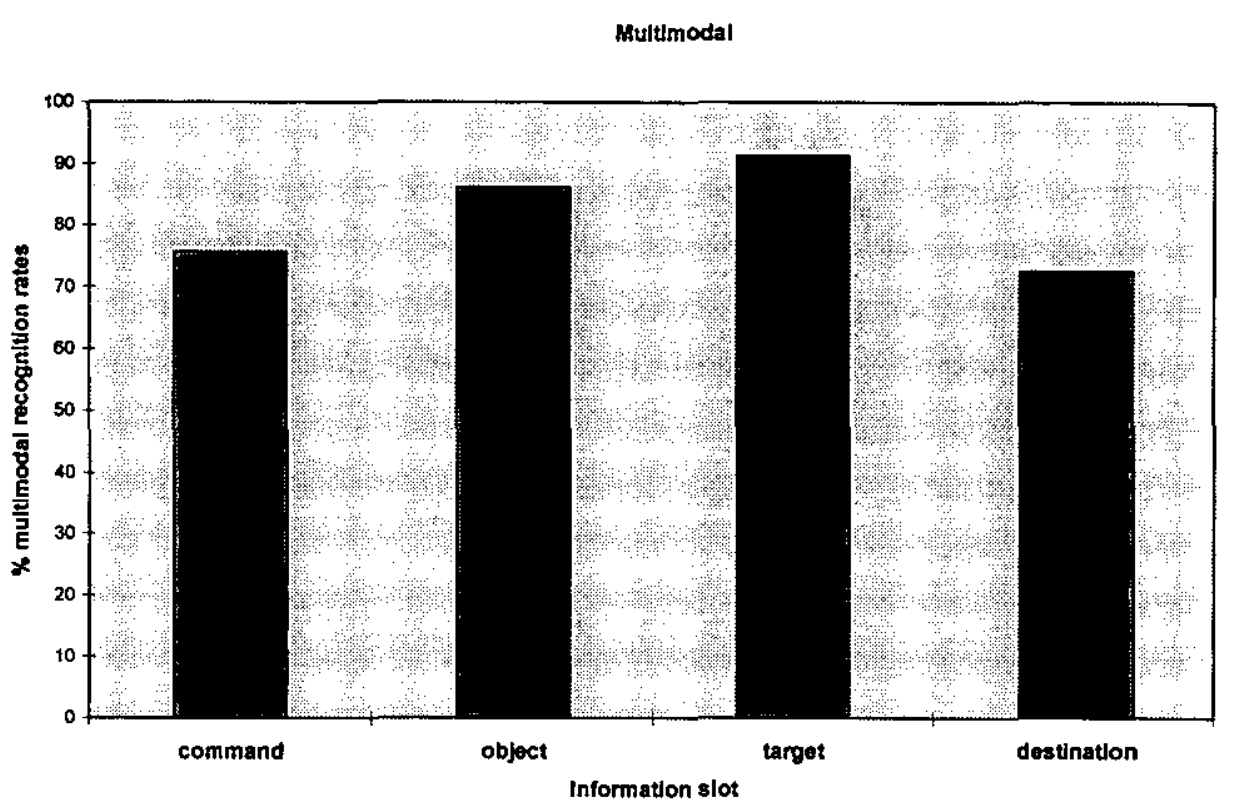

Figure 54: Average multimodal recognition results for command, object, target and destination types

The results show distinct differences between the slot types. In the speech recognition results, the commands were recognised most successfully, followed by the object. The recognition results fall in the recognition of target numbers, and the destination number recognitions show the lowest rate of all the cases. This result agrees with earlier observations that speech command and object input was handled well by the system, while difficulties existed in the handling of speech number input.

In the gesture recognition case, the recognition of commands is low. Object and target number recognition are higher, and target recognition gives the highest result. The recognition of destination numbers is better than commands but lower than object or target.

In the multimodal case, it is expected to see that multimodal recognition rates are an improvement over gesture recognition rates. The multimodal results show the same trend as the gesture recognition results, where command gives the lowest result, destination gives the third highest result, object recognition is second 
highest and the target results are the highest. Generally, the multimodal rates are higher.

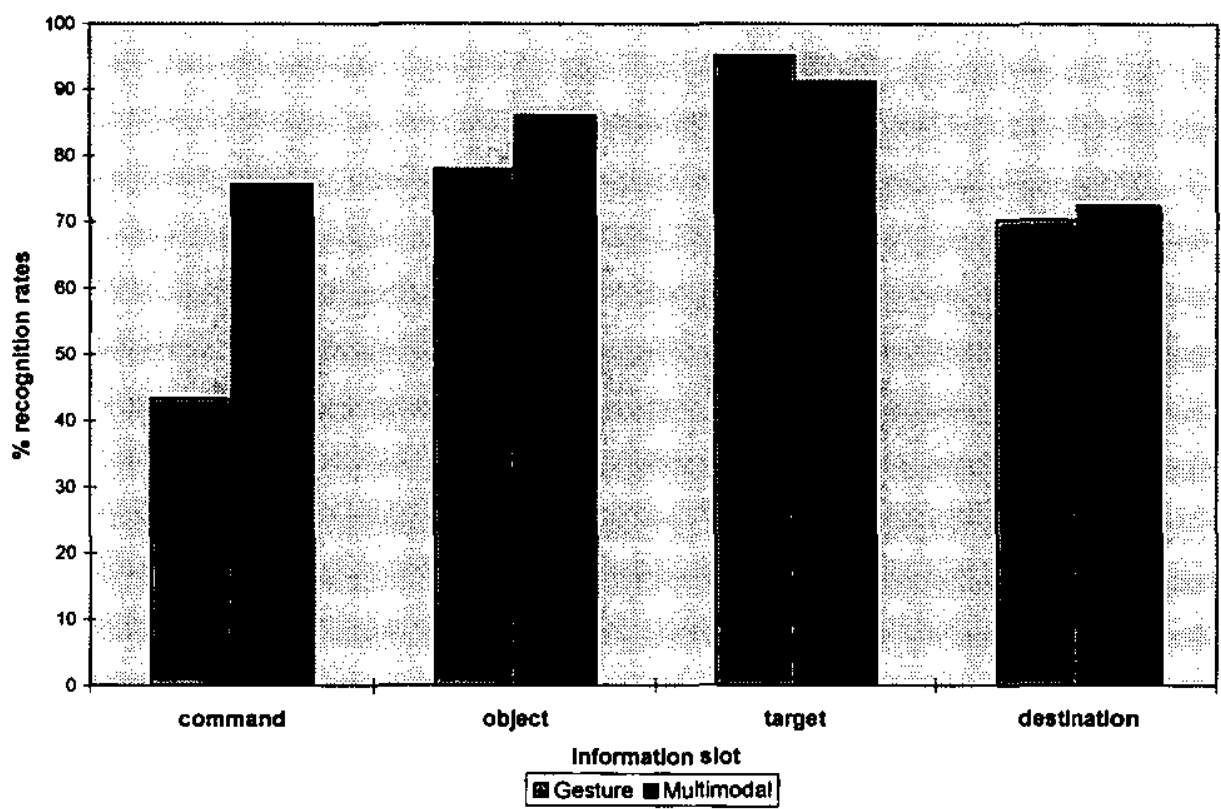

Figure 55: Comparison of gesture and multimodal recognition results for command, object, target and destination types

When gesture and multimodal recognition results are compared (Figure 55), it shows that multimodal recognition rates are higher than gesture rates in three cases: command, object and destination. The multimodal rates are marginally higher than gesture rates in the object and destination cases, and marginally lower than gesture rates in the target case.

In the command case, the multimodal rates are substantially higher than the gesture rates, as the gesture rates are much lower than in the other cases. The command results show that the multimodal rates are statistically significantly higher than the gesture rates $\left(\chi^{2}=6.8612, \mathrm{p}<0.01\right)$. The gesture recognition rate of crosses in the final multimodal system was problematic, due to the slight time delay introduced to the processing by the use of the truth maintained blackboard system. (In Table 26, Appendix A, the gesture recognition rate for crosses across the whole experiment is $0.00 \%$ ). The set time-gap allowed between gestures for components to be interpreted as cross parts was 0.8 seconds. 
The time taken from the end of one gesture until the system was ready to receive another gesture was greater than 0.8 seconds. During this time, the gesture parameters were sent to the truth maintenance system, a conclusion produced, which was sent back to the gesture recognition system (Chapter 6). After this conclusion had been processed by the multimodal system, another gesture was then expected by the system, if a cross had been requested. All cross recognitions failed as the time-gap was not adapted to take account of the delay.

The object recognition rates are statistically significantly different from one another, with multimodal rates higher than gesture $\left(\chi^{2}=9.800, p<0.01\right)$. The multimodal recognition rate for target numbers is slightly lower than the gesture rate, and the rates do not show significant statistical difference $\left(\chi^{2}=0.1020\right.$, $\mathrm{p}=0.7494)$.

The multimodal recognition rate for destination numbers is slightly higher than the gesture recognition rate. The gesture and multimodal rates are not significantly different $\left(\chi^{2}=0.9184, \mathrm{p}=0.3379\right)$. The interpretation rates of the destination numbers over speech, gesture and multimodal are lower than any of the other categories of command, object, and target numbers. These results confirm that the multimodal interpretation of numbers is less successful than the interpretation of command and object information.

The low rates for the target and destination numbers can be attributed to the difficulty in categorising whether the numbers were targets or destinations. The difference was determined using knowledge about other words in an utterance (e.g. "from" before target numbers, and "to" before destination numbers) as well as knowledge about the start and end of gestures. Frequently, "to" was confused for " 2 " by the speech recognition system, which contributed to the low speech recognition rates for destination numbers. "From" was also recognised poorly at times by the speech software, which made interpretation of target numbers in speech, based on this context knowledge, difficult. This was a difficulty unique to the parsing of the numbers. The other cases of command, object and target did not have similar parsing difficulties to affect the overall recognition. 
Therefore, by analysing the recognition data by the information type (command, object, target and destination), this has revealed that two main cases, command and object gave multimodal rates which were significantly higher than gesture rates. There is no statistical difference between the target and destination cases. These results are consistent with the earlier results from the redundancy analysis (Section 8.6) where multimodal interpretation of the target and destination input appeared to be poor. The command and object interpretation has been shown to be robust.

\subsubsection{Reinforcement and resolution of meaning with redundancy}

The data from the multimodal experiment was also analysed for evidence of reinforcement of meaning and resolution of ambiguities through the presence of redundancy. Recall that from the objectives of analysis (Chapter 1), cases of interest included investigation of how often redundancy reinforced meaning (speech and gesture information agreed), and how often redundancy resolved meaning (speech and gesture information disagreed).

Additionally, these cases determined how the speech and gesture were combined into a single multimodal representation in the integrator. Therefore, the success of these cases in resolving input into a correct multimodal interpretation was directly related to the overall multimodal recognition rates.

The data was analysed to determine the percentage of correctly interpreted multimodal representations for command only, object only, target only and destination only input in three possible cases. 
These cases were:

- final speech and gesture choices agree

- final speech and gesture choices disagree

- $\quad$ only speech-alone information available (no gesture) or vice versa: only gesture-alone information available (no speech)

This raw data is shown in Table 28, Appendix A. The average figures of this data are shown in Figure 56.

Average percentage multimodal scores

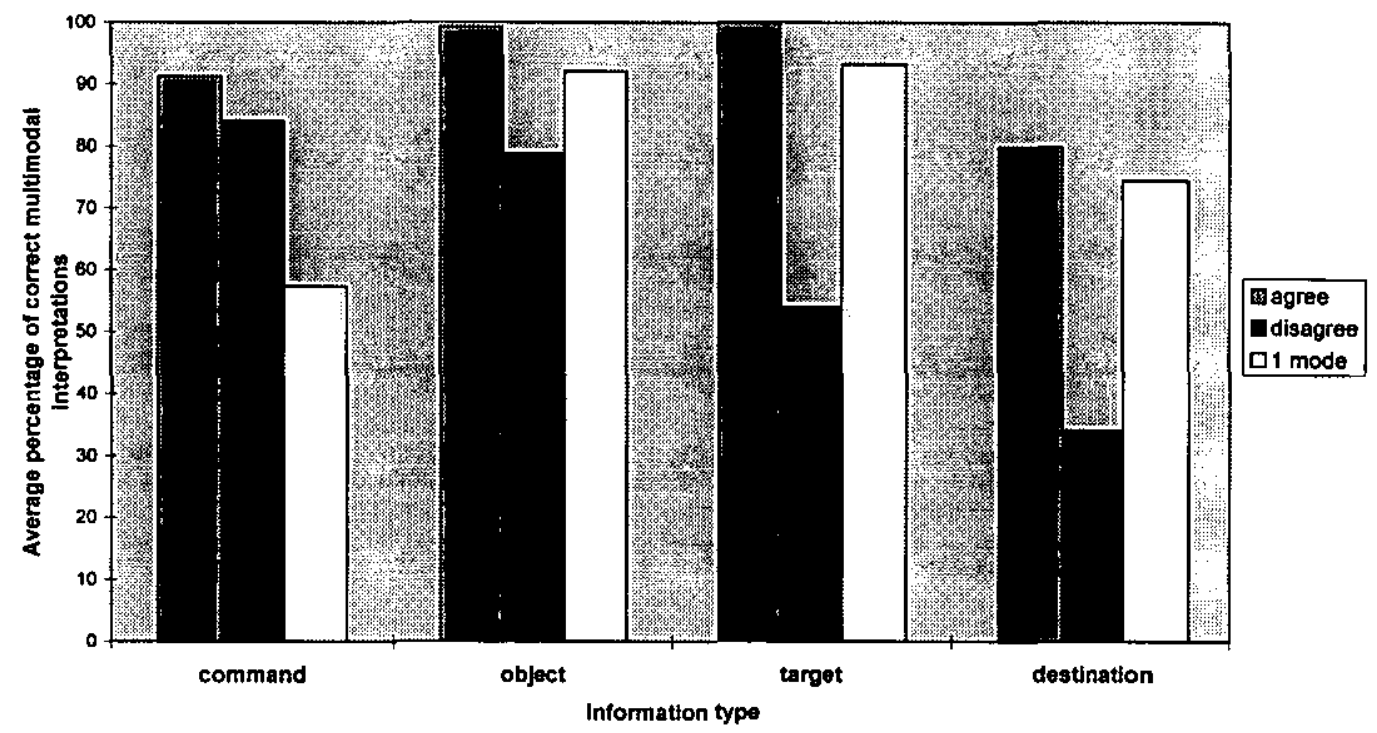

Figure 56: Average percentage of correct multimodal interpretations

The average rates of correct multimodal interpretations for command input alone are: agrees $=91.2 \%$, disagrees $=84.1 \%$, one mode $=57.3 \%$. This can be observed in the graph, where the one mode results are lower than both agreement and disagreement cases in 28 out of 30 cases. These figures are statistically significantly different, $\chi^{2}=52.48, \mathrm{p}<0.0001$.

The average rates of correct multimodal interpretations for object input alone are: agrees $=99.2 \%$, disagrees $=78.8 \%$, one mode $=92.1 \%$. In 23 out of 30 cases, the disagreement cases are lower than both agreement and one mode cases. These figures are statistically significantly different, $\chi^{2}=50.83, p<0.0001$. 
The average rates of correct multimodal interpretations for target number input alone are: agrees $=99.8 \%$, disagrees $=53.9 \%$, one mode $=93.2 \%$. In 23 cases out of 30 , the disagreement cases are lower than both agreement and one mode cases. These figures are statistically significantly different, $\chi^{2}=65.12, \mathrm{p}<0.0001$.

The average rates of correct multimodal interpretations for destination number input alone are: agrees $=79.9 \%$, disagrees $=34.1 \%$, one mode $=74.4 \%$. In 28 cases out of 30 , the disagreement cases are lower than both agreement and one mode cases. These figures are statistically significantly different, $\chi^{2}=53.39$, $\mathrm{p}<0.0001$.

The graph indicates that the best multimodal interpretations occurred when the speech and gesture modes agreed. This shows that redundant information successfully reinforced meaning in the majority of cases. When ambiguities occurred between speech and gesture, i.e. the information from each mode disagreed, the multimodal interpretation rate was lower than when the modes agreed. This is a reasonable result to expect given that the method of creating a multimodal representation, and attempting to resolve the ambiguity, was dependent on arbitrary confidence weights. Therefore, the 'wrong' choice of mode could be made because that mode had a higher weighting. The ANOVA results for the individual inputs, in the speech, gesture and multimodal cases are shown in Table 23. 


\begin{tabular}{|c|c|c|c|}
\hline information type & mean rank & chi-square, $\chi^{2}$ & significance level \\
\hline command & $\begin{array}{r}\text { agree }=65.58 \\
\text { disagree }=52.60 \\
\text { one mode }=18.32\end{array}$ & $\chi^{2}=52.48$ & $p<0.0001$ \\
\hline object & $\begin{array}{r}\text { agrees }=71.75 \\
\text { disagrees }=25.62 \\
\text { one mode }=39.13\end{array}$ & $\chi^{2}=50.83$ & $p<0.0001$ \\
\hline target & $\begin{array}{r}\text { agrees }=72.97 \\
\text { disagrees }=20.00 \\
\text { one mode }=43.53\end{array}$ & $\chi^{2}=65.12$ & $p<0.0001$ \\
\hline destination & $\begin{aligned} \text { agrees } & =63.83 \\
\text { disagrees } & =17.50 \\
\text { one mode } & =55.17\end{aligned}$ & $\chi^{2}=53.39$ & $p<0.0001$ \\
\hline
\end{tabular}

Table 23: Results of Kruskal-Wallis one way ANOVA for agree, disagree and one mode cases for command, object, target and destination input

Over all information types, the average interpretation rates are shown in Figure 57.

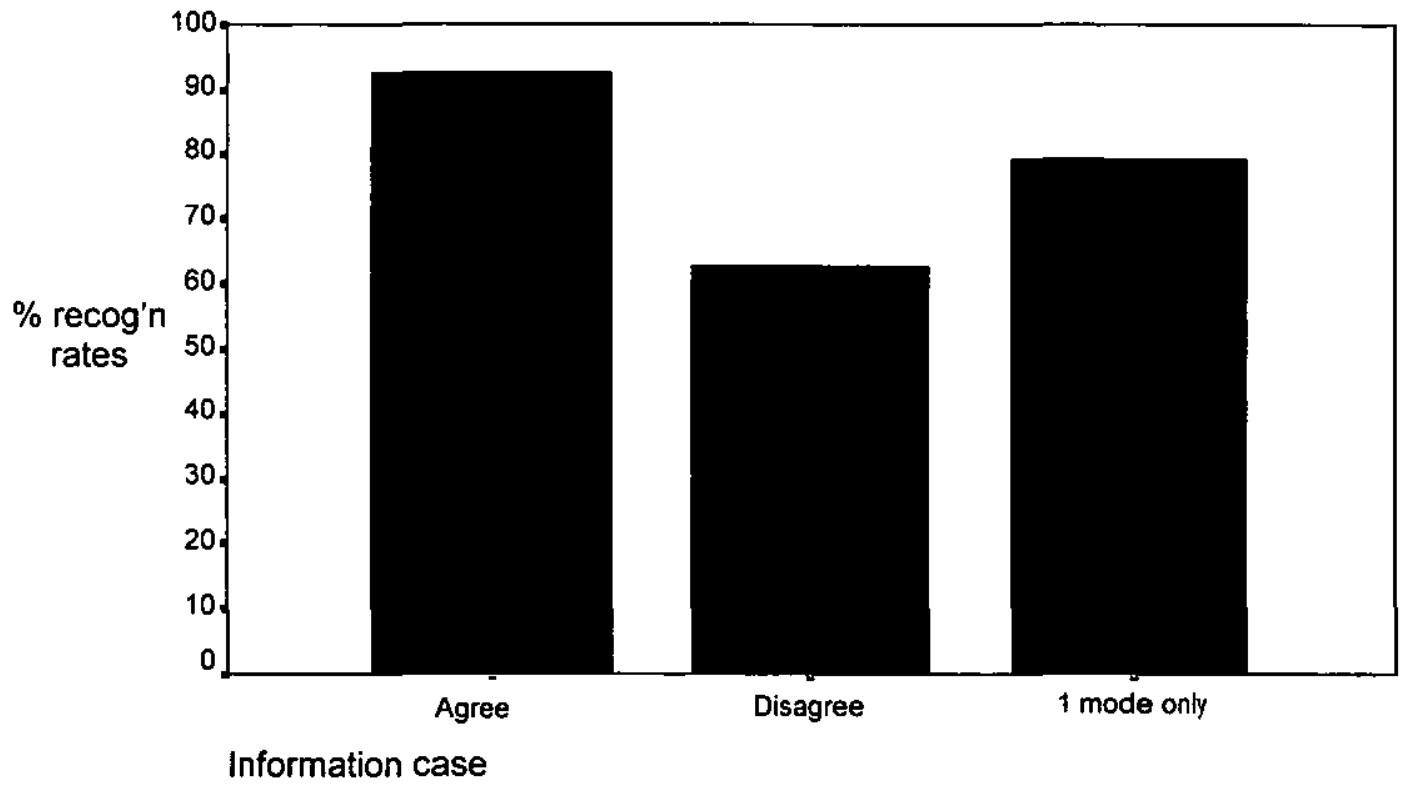

Figure 57: Average multimodal interpretation rates for agree, disagree and one mode cases over all input

The average rates across all input (command to destination) indicate that overall, when speech and gesture information agreed, this produced the statistically 
significantly highest multimodal recognition rates $(92.54 \%$ ) (Figure 57 ). The disagreement case gave the lowest rates $(62.71 \%)$, while the one mode only case generated multimodal rates which were between the agree and disagree cases (79.24\%). The three rates were significantly different from one another $(\mathrm{F}(2,357)=65.1144, \mathrm{p}<0.00001)$.

\subsection{Summary}

Empirical results have been presented in this chapter from an initial experiment, conducted using 8 subjects. A further experiment using 30 subjects generated substantial results about the role of redundancy across input modes. Multimodal rates were found to be statistically significantly higher than both speech and gesture rates for average recognition rates across complete tasks, at the $100 \%$ redundancy level.

An analysis of redundancy levels and multimodal recognition rates found that although there was no significant linear correlation between the figures, more complex relationships existed. In particular, the highest multimodal rates were generated where redundant information was present in cut, copy and paste actions (especially in the presence of redundant command information). The multimodal recognition rates rise as the amount of redundant information between the modes increases. Redundant information was less successfully exploited by the multimodal integrator in move tasks. This has been attributed to the difficulty which the integrator had in determining the difference between target and destination numbers. The lack of improvement in the multimodal recognition rates in move tasks has also been attributed to the high gesture recognition rates in these cases which we suggest are difficult to improve upon. Gesture recognition of the move gestures (lines of vertical, horizontal, positive and negative gradient) was more successful than the recognition of the cut, copy and paste gestures (crosses, circles and ticks) as they are simpler gestures.

Analysis of the recognition rates from the individual components of the tasks, the command, object, target and destination information, revealed that the 
multimodal interpretation was statistically significantly higher than the gesture interpretation in the command and object cases. In the target and destination cases, there was no significant improvement in the multimodal rates. This confirms the expectations that while command and object interpretation was robust, the integrator encountered difficulty in the interpretation of the target and destination numbers.

The multimodal recognition rates were also analysed in three cases: when speech and gesture information agreed, when speech and gesture information disagreed, and when only one mode was available (through recognition errors, or lack of redundancy across modes). The highest multimodal recognition rates were generated when speech and gesture information agreed. This shows that redundant information successfully reinforced meaning in the majority of cases (Figure 56, pp198), and also resolved meaning in some ambiguous cases, although less frequently in the disagree case than the agree case. 


\section{Section 4}

\section{Summary \& Conclusions}




\section{Chapter 9}

\section{Conclusions \& Future Work}

\subsection{Introduction}

This chapter discusses the conclusions of the research. A summary of the thesis is first presented, followed by discussion of the results. A review of the thesis considers the improvements which could be made, and analyses possible future work in the area. A comparison of the initial aims and objectives of the thesis with the actual achievements is also presented.

\subsection{Summary of thesis}

The thesis began by outlining a characteristic of human to human communication, the degree of redundancy in communication. The property of redundancy provides a flexible and rich combination of information in communication, which supports the interpretation and transfer of information in conversations. This benefit of redundancy has also been noted as desirable in human-computer communication and interaction. It has been observed that multimodal recognition 
rates can be higher than their unimodal counterparts, attributing the improvement to the provision of redundancy.

\subsubsection{Activities and Initial Work}

The initial motivation for this thesis was to investigate the exploitation of redundant information in a simple system, without the best possible quality of input recognition. This approach would emphasise redundant information, and the use of task, system and context knowledge, to create improvements in multimodal recognition. An inexpensive, commercial speech recognition system was chosen to handle speech input, and it was decided to implement a simple two-dimensional gesture recognition system.

\subsubsection{Two-dimensional gesture recognition system}

The two-dimensional gesture system was developed to produce parameters which characterise a set of chosen gestures. The gestures which it recognised were: tick, circle, cross, and a line (with various gradients). In the initial system, the gesture parameters were broad enough to capture elements of each gesture, but it was difficult to determine the bounds of the parameters for each individual gesture. Some form of description or classification of the gestures was therefore required. Rule induction was identified as a flexible way of generating classification models, and in particular, production rules.

A set of experiments was subsequently conducted to generate gesture data for classification by the rule induction system. The production rules generated from the rule induction system, showed that there was a large degree of variability in the gesture data from individual users. The production rules captured this variability and were also general enough to classify the standard gesture characteristics. A method was then required which would allow the best choice from the production rules to be made, on more than an ad-hoc basis to maintain consistency across gesture recognition. 
These rules were therefore used as the knowledge bases of a truth maintained blackboard system, as part of the final gesture recognition system implementation. The truth maintenance system had the capability of retaining several views of a problem, which in our case is gesture recognition. As a result the system could sustain a number of recognition possibilities for a gesture, thus providing a choice of gesture suggestions to the recognition system.

\subsubsection{Speech system}

The commercial speech software used was a discrete speech recognition system which provided parameters about the word recognised, other recognition possibilities and confidence ratings for these possibilities. The speech system was programmed with an appropriate set of vocabularies and the speech recognition capabilities were investigated in order to provide speech aware properties to the final multimodal input system.

\subsubsection{Multimodal System}

The multimodal input system was developed using the input modes of speech and two-dimensional gesture, which provided a simple and inexpensive method of investigating redundancy between input modes. The multimodal system exploited the information parameters and characteristics of both speech and gesture, integrating this information into a single multimodal representation, using a multimodal integrator designed for the system. The integrator used an information slot structure similar to conceptual dependency structures, to represent the information from the speech, gesture and combined multimodal information.

This system was used in experiments, with constraints on the order and type of input, to analyse the role of redundancy between the input modes. An initial experiment to test the system showed that multimodal rates were significantly higher than gesture recognition rates for eight subjects. The format of the experiment requested that users provide sequential speech and gesture input not 
simultaneous), to perform manipulation tasks of coloured icons around a grid. Users were asked to follow on-screen guides which generated utterances for them to repeat, and gestures for them to perform. Possible task commands were cut, copy, paste and move, and complete tasks contained command, object and location (target and/or destination) information.

The microphone was constantly switched on in this initial experiment, and therefore noises between utterances were detected by the microphone. This reduced the speech recognition rates, as these noises (e.g. coughs, sneezes, other speech) were processed as part of the intended input by the system, which attempted to resolve this input as part of the multimodal task.

Analysis showed that the speech, gesture and multimodal rates were significantly different from one another. It was expected to find a difference between the speech and other rates, as speech input was made over different task content (a smaller task set). The multimodal recognition rates were statistically significantly higher than the gesture recognition rates. The average speech recognition rate for this experiment was $51.8 \%$. The gesture rate was $75.7 \%$, and multimodal rate was $82.9 \%$. This initial experiment confirmed that the data was being generated and recorded properly. Subsequently, a larger study with thirty subjects was undertaken, giving a larger sample to draw conclusions from.

\subsection{Main Achievements}

\subsubsection{Probing the role of redundancy}

An investigation of redundancy between the input modes of speech and twodimensional gesture has shown that redundancy does have a significant effect on multimodal recognition rates. 


\subsubsection{Main Experimental Results}

A multimodal experiment using thirty subjects was conducted using the same format as the initial experiment, with one change to the operation of the speech system. The microphone, which had been constantly left on in the initial experiment, was switched off between utterance input in the main experiment. This ensured that other noises were not detected and processed by the speech system, and allowed better performance of the speech recognition system within the multimodal application.

The speech, gesture and multimodal recognition data generated from the main experiment was analysed for the following figures:

- $\quad$ average speech recognition figures across all complete tasks

- $\quad$ average gesture recognition figures across all complete tasks

- $\quad$ average multimodal recognition figures across all complete tasks

The data was also analysed for the following:

- relationships between the amount of multimodal gain and unimodal recognition rates

- relationships between the levels of redundancy and the multimodal recognition rates

- relationships between the difference of recognition success between modes and the multimodal recognition rates

- evidence of the reinforcement and resolution of meaning through the presence of redundancy by calculating

- percentage of correct multimodal interpretations when speech and gesture information agrees

- $\quad$ percentage of correct multimodal interpretations when speech and gesture information disagrees

- percentage of correct multimodal interpretations when information from only one mode is available (due to lack of redundancy between speech and gesture modes, non-recognitions and mis-recognitions in one mode) to compare with reinforcement and resolution figures

The main experiment generated substantial empirical results. The multimodal recognition rates were significantly higher than both the speech and gesture recognition rates. 


\subsubsection{Redundancy Analysis}

An analysis of the relationship between redundancy levels and multimodal recognition rates was then performed. Redundancy was measured as the amount of information which was constantly present in the gesture information, and was repeated in the speech information. The five main redundancy levels were expressed as the percentages of $25 \%, 33 \%, 66 \%, 75 \%$ and $100 \%$. Within these levels were a total of twelve sub-levels, which represented the type of information repeated within the redundancy level, a combination of command, object, target and destination information. The twelve sub-categories were C25, 025, D25, C33, O33, T33, CO66, CT66, COD, COT75, COT100, and COTD (Table 17, pp 178).

The relationship between redundancy and multimodal rates, was influenced by two main factors: the task type, and the type of information involved. This was revealed through various analyses. For instance, a test for linear correlation between the five main redundancy levels and multimodal recognition rates did not reveal a significant linear relationship between the variables. Investigation of other relationships between redundancy and multimodal recognition rates was therefore required.

These showed that multimodal rates were statistically significantly higher than speech and gesture rates in the $33 \%, 66 \%$ and $100 \%$ cases. In the $25 \%$ and $75 \%$ cases there was no significant difference between speech, gesture and multimodal rates. In particular, the $25 \%$ and $75 \%$ redundancy levels did not show an improvement in the multimodal recognition rates over gesture rates. This result was attributed to the fact that these levels represented move actions, which presented a larger number of ambiguities for the integrator resolve, as there was a higher number of slots to fill. Another factor was the successful gesture recognition rates which were consistently at the $80 \%$ level for move gestures (compared to approximately $60 \%$ in the cut, copy and paste tasks). Move gestures (lines of various gradients) were easier to recognise than the more complex cross, circle and tick gestures of the other tasks. Therefore, the multimodal recognition quality showed an improvement of the gesture rates in 
cut, copy, and paste actions, while it was difficult to improve upon the already high gesture rates in the interpretation of move actions.

For cut, copy and paste tasks, all of the sub-levels of redundancy produced significantly higher multimodal scores, most noticeably in the $\mathrm{C} 33, \mathrm{CO}$, and COT100 cases which contributed to the high multimodal rates in the overall redundancy categories of $33 \%, 66 \%$ and $100 \%$.

The multimodal rates were then analysed to determine which redundancy level gave the highest multimodal recognition rate. This showed that the $66 \%$ and $100 \%$ redundancy levels gave the highest rates. This analysis also showed that recognition of those tasks which included redundant command information was significantly better than those tasks without redundant command information present. An exception to this again were the redundancy levels for the move tasks, COT75, COD, and COTD.

The results show that it is the type of redundant information present and how it is used which has more effect than the volume of redundant information. Although the cases with a higher redundancy level did produce higher multimodal recognition rates, the exception to this pattern were those cases involving the move actions. Two distinct task types, a set with only one location slot (cut, copy and paste actions) and another set with two location slots (move actions) gave distinct results. The interpretation of the numbers for the location slots was poor, and this decreased the multimodal recognition rates in these move cases. The result also suggests that the presence of redundancy information has most effect at particular levels of unimodal performance, namely where it is possible to create a significant improvement in the unimodal rates.

\subsubsection{Task Recognition}

An analysis of the recognition rates of the components of each task, the command, object, target and destination information was carried out. This analysis showed that the multimodal rates were statistically significantly higher 
than the gesture rates in the interpretation of the command and object and destination information. There was no significant difference between the modes in the target and destination cases. The multimodal rates were lower than the gesture rates in the target cases.

\subsubsection{Reinforcing and Resolving meaning}

Finally, the multimodal recognition rates were examined in three cases: when speech and gesture information agreed, when speech and gesture information disagreed, and when only one mode was available (through recognition errors, or lack of redundancy across modes). These rates were obtained for command input, object input, target input and destination input alone.

When redundant information was present across speech and gesture, and when that information agreed from both modes, the correct multimodal interpretation was made in $92.54 \%$ of cases. When the redundant information did not agree, the redundancy still produced the correct multimodal interpretation in $62.71 \%$ of cases, which was lower than the rate when information from only one mode was present $(79.24 \%)$.

Therefore, when redundant information was correctly interpreted, it reinforced meaning in the majority of cases. However, when ambiguities were present in the input information, the redundant information was less successful at resolving (although still a relatively high figure of more than half of cases). Broadly, both cases of resolution and reinforcement benefited from the presence of redundant information.

\subsection{Discussion}

\subsubsection{Comparison of approach with others}

The performance of the multimodal input system presented here compares very favourably with other similar approaches. Limited performance data is available 
about such multimodal input systems. For those systems where performance data is available (Vo \& Wood, 1996) the multimodal recognition rates, and the performance of the multimodal integrator were poor in comparison to the system presented here. The multimodal system developed by Vo \& Wood gave average multimodal recognition rates of $35 \%$, as compared to the average rate of $82 \%$ generated by the system presented here.

There is no other reported work of the manipulation of levels of redundancy between modes, to analyse multimodal recognition rates in this context.

\subsubsection{Design of multimodal systems}

The evidence that redundancy helps to provide multimodal recognition rates which are higher than unimodal recognition rates, based on a simple multimodal integrator algorithm, suggests that systems which use similar integration approaches will benefit from the presence of redundancy. The presence of redundancy when used with task knowledge, improved the effectiveness.

\subsubsection{Thesis in Retrospect}

A number of improvements in the development of the system could have been performed. These improvements would have allowed analysis of other factors in the performance of the system.

\subsubsection{Use of task knowledge}

The context and system knowledge used by the integrator was embedded within the system framework. More flexibility and further analyses could have been performed if a more modular structure had been developed, to produce a separate context knowledge module as part of the entire framework. With a modular structure, it would have been possible to more readily change the system and context knowledge, in cases where the application or task type was changed, just by adding or modifying the module. 
Expansion of the capabilities of the context knowledge base would also be desirable. For instance, the context and system knowledge was used to check the validity of task details, such as chosen object validity on particular grid numbers, and the validity of chosen destinations (e.g. was another object already placed there?). However, the context knowledge was not used to explicitly check chosen commands. Knowledge about the tasks could have been used to make guesses about commands in cases of ambiguity (e.g. if the task was performed on an empty grid, then the command is a paste or a move; if the task is on a non-empty grid, then the command is a cut or a copy).

\subsubsection{Use of rule induction}

More consistency could have been introduced in the analysis by performing rule induction on the speech data as well. Rule induction was very useful in capturing the variability of the gesture data, providing a flexible classification model. Its application to speech was rejected for the work described here, as the speech parameters were fairly limited in scope. Although the parameters included other recognition possibilities, there was a lack of actual word parameters, such as phonetic information. Future work could address this lack of speech parameters and investigate the use of rule induction on the speech data.

The use of rule induction on the gesture data proved successful as a form of classification. The nature of the gesture data was ideally suited to this form of classification, as there were distinct categories (the gestures), several parameters describing the categories (the gesture characteristics) and a fairly large data set to work on (the data from the experiments). The rules generated from the rule induction were descriptive, and were easy to follow and apply to gestures. Such a classification would not have been possible on an ad hoc basis, or through other methods such as statistical linear classifiers, which classify through arithmetic properties. The average recognition rate from the system was $80 \%$ using the rules. 


\subsubsection{Use of truth maintained blackboard system}

Another strategy which could have been exploited was the use of the truthmaintained blackboard system for integrating the speech and gesture input. This approach was not used in the system because of the embedded context knowledge, and the use of time-stamps which was difficult to implement in the Prolog framework.

The use of the truth maintenance system in the gesture recognition system proved successful, as it was an ideal way to interpret the production rules. The capability to provide several possible gesture candidates in response to a set of gesture parameters was ideal, as these possibilities were used in the final multimodal interpretations along with the speech recognition possibilities.

\subsection{Future Work}

\subsubsection{Improving Integration Algorithm}

The integration algorithm could be improved in a variety of ways.

As separate speech and gesture input systems were used, the associated weighting systems were independent of one another, and hence arbitrary in any comparison. If the same weighting systems were used for each mode, the comparison of speech and gesture possibilities would be more effective.

\subsubsection{Mis-recognitions}

Mis-recognised cases can be expected from the system. The integrator was programmed to make a best guess about the intended actions, based on the weightings given to the inputs by the speech and gesture systems. Due to the use of independent speech and gesture recognition systems, the rating mechanisms were different. Therefore, as well as being arbitrary within the individual systems, any comparison of the weights was also arbitrary. Hence it would be 
reasonable to expect this system to choose an option (based on the weights) that was incorrect, simply because the 'wrong' answer had the higher weighting.

Another factor in mis- and non-recognitions was the dependency of the complete multimodal combination on passing a total validity check. The validity check analysed the proposed multimodal combination in total, instead of considering possibilities slot by slot. This provided an efficient way of checking the complete interpretation and was less time consuming than modular checking of each slot before combination. As the total multimodal combination had to be estimated to be correct, one or more slots that may have contained valid (and correct) values would have been discarded in the search for the best overall combination. Another possible validity check could have filled each slot with a possibility, checked that option for validity, and then attempted combinations of this slot with other slots, ending with a final validity check of the complete combination. This may have taken longer but may have provided more thorough validity checking and use of task knowledge.

For instance, there was the possibility of one particular multimodal slot being filled with the correct value from one or both modes (e.g. the command slot), in combination with a value for another multimodal slot which was incorrect (e.g. the object slot). This combination would then be rejected by the validity checker if it was known by the system that the particular object was not at the indicated target. When another combination was formed, the correct value for the command slot was changed for a second (or other) choice, whilst the incorrect object slot was filled with another choice. Possibly, in this iteration, the command was incorrect but the object was correct.

\subsubsection{Improving use of information}

The design of the multimodal integrator was based on a linear search through a maximum of 100 possible speech and gesture combinations. The search stopped when the first valid combination of parameters from speech and gesture was identified. This strategy did not exploit all the available information however. 
Choosing the first valid option gave an efficient strategy but overlooked further information that may have been available in the non-1st choices of the recognition possibilities.

Consider the following example:-

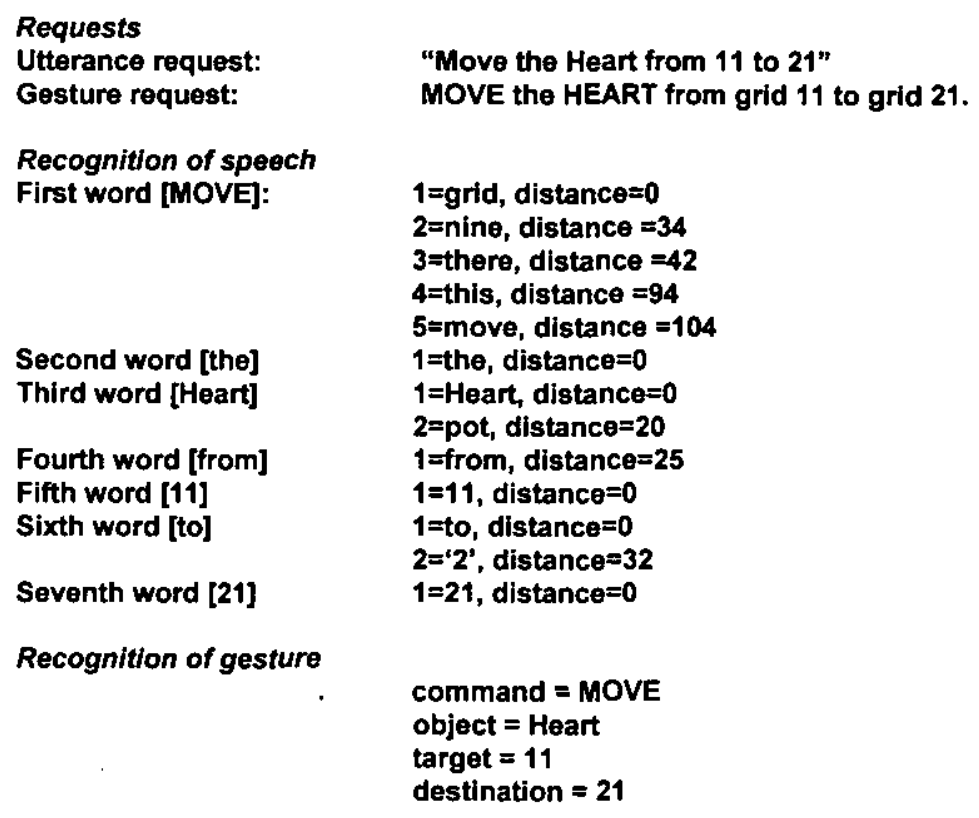

The distance value represents how far in accuracy the speech recognition believed the recognition possibilities were from the first choice, and is an inverse confidence rating (the lower the distance value the more confident the system is about a possibility). The gesture was interpreted correctly as were the object and location information slots.

When the first speech possibility is interpreted (grid), the speech information slots are filled from the first choices from the recognition possibilities and have the following values:

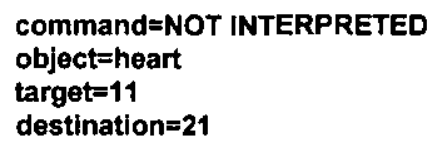

Similarly the gesture information slots are filled from the first choice recognition possibilities and have the following values: 
command=move

object=heart

target=11

destination $=21$

When the speech and gesture slots are combined to fill possible multimodal slots the multimodal command slot becomes move, taken from gesture, object is heart (taken from both), target is 11 (taken from both modes) and destination is 21 (taken from both modes). Then when the combination is checked for validity against current context knowledge (e.g. is there a heart already present on grid 11 which can be moved?), the combination passes and the system decides that a valid combination has been found.

The final multimodal command of move has been taken solely from gesture, a less certain conclusion than if speech had also provided some information towards this conclusion. The object and location slots have been filled from both speech and gesture adding a greater certainty to these slots. If all of the speech possibilities had been examined to find an agreement between speech and gesture, the 5th recognition possibility of speech could have been identified as an agreement for the first gesture choice, and given a stronger conclusion for the final multimodal command.

Scope for reinforcement of this kind would be especially useful at times when there is greater ambiguity and doubt about the possible conclusion. This type of further development would lend further 'intelligence' to the integrator and it could be speculated that the multimodal recognition rates could be improved further.

\subsubsection{Perturbing input data}

A further development of this experiment to generate an additional study of redundancy with the same data, would be a perturbation of the data with the addition of noise. Refined perturbation could be performed, where the raw input data (e.g. co-ordinates of the gesture data) could be randomly adjusted to modify the input. The data could then be re-analysed based on the noisy (modified) data to determine the effect of the noisier input. This refined perturbation, using the 
raw data could only be performed on the gesture data, as the basic input information (the co-ordinates) are available. The raw speech data was not available since a commercial speech recognition system was being used. Therefore, coarse perturbation of the speech data would be possible just by randomly changing some of the numerical representations of the stored speech results.

This could be considered less appealing than the refined method, but still valid to analyse the behaviour of redundancy. Thus analysis, with perturbation of the statistically better performing mode could be achieved. The approach could be justified when an early consideration of redundancy is revisited: that especially at times of poor input quality the presence of an additional mode gave recognition gains.

\subsubsection{Freely interactive multimodal system}

An initial version of the multimodal input system was developed before the constrained experimental version. This version of the multimodal system was in the form of a game, where speech and gesture could be used in any order and combination to operate the game. The game, similarly to the format of the experiments, involved the manipulation of coloured shapes around a grid.

In this version, input could be made in any order: speech/gesture, gesture/speech, speech alone, gesture alone. Experimental observation may provide results on the effects of reversing the order of modes, confirm user preferences, and examine whether the mode choices affected recognition rates. 


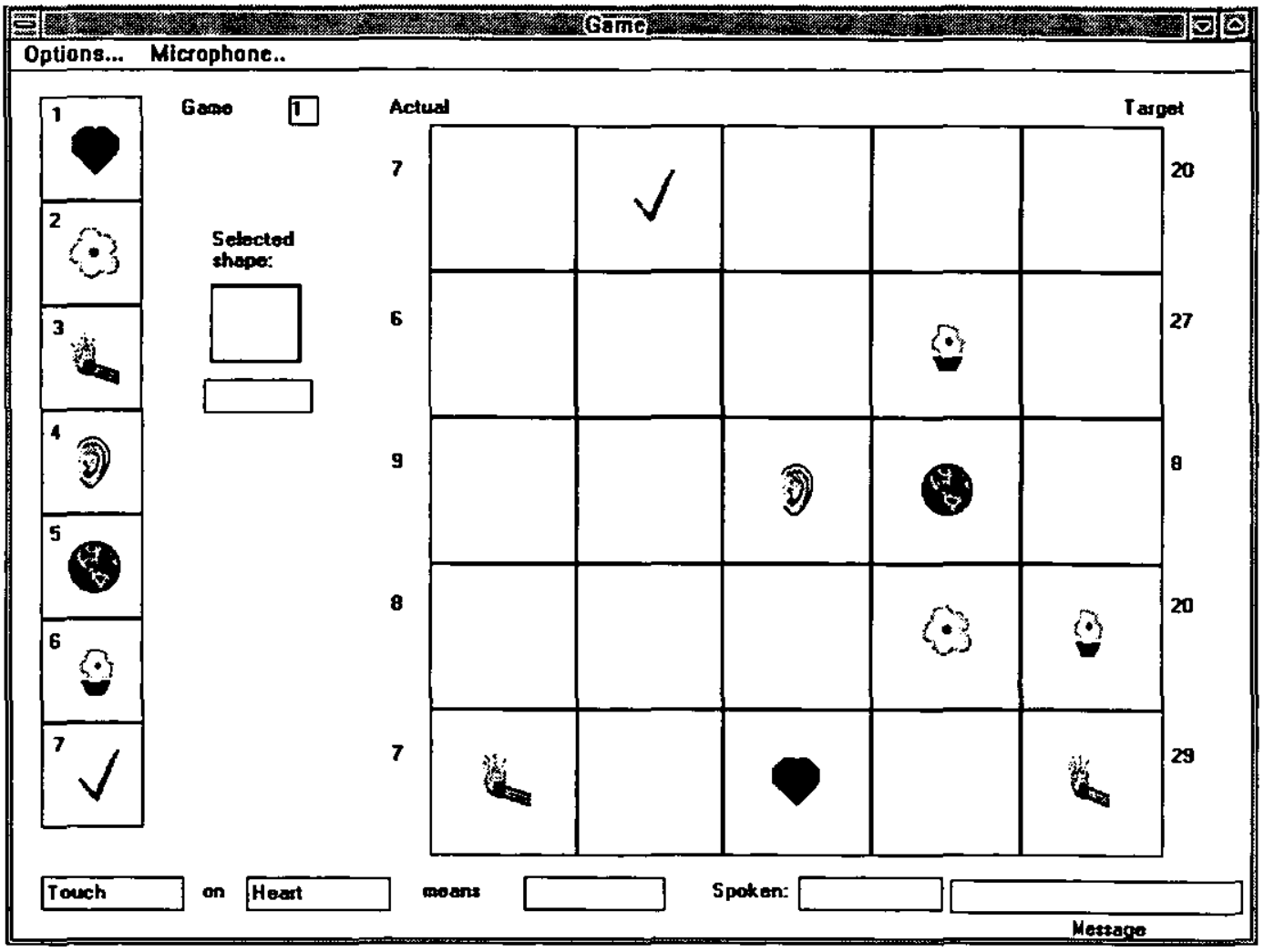

Figure 58: Free interactive game application of multimodal input system

Analysis of subjects whilst using the game would give details of the use of multiple modes by humans, such as temporal co-ordination, information on preferences for modes, and preferences on the repetition of speech in multimodal systems.

\subsubsection{Epilogue}

A simple multimodal system has been created for this thesis, using the input modes of speech and two-dimensional gesture. Initial investigation of the individual input systems showed that they each had reasonable recognition performance. The performance of the multimodal system using these component systems, was also successful, and task recognition was better in the multimodal system.

The experimental performance of the multimodal system was very encouraging. Average multimodal recognition rates across all subjects and all redundancy levels were approximately $80 \%$, and in particular redundancy levels a multimodal 
recognition rate of $92 \%$ was achieved. These rates compare very favourably with other reported figures available. There are reported systems, where the multimodal recognition rate has not improved upon the unimodal recognition rates (Vo \& Wood, 1996).

The empirical data gained about the role of redundancy is novel and shows that the presence of redundant speech information has improved upon the interpretation of the tasks presented here, creating multimodal recognition rates which were higher than the comparable gesture recognition rates. In particular, the effect of redundancy was seen most in simple tasks, where the gesture recognition rate was reasonable but scope existed for improvement. The effect was less noticeable in the more complex move tasks, where gesture recognition rates were higher, and the multimodal interpretation was more difficult to perform. This was due to the different information content between the tasks. The presence of redundant command information produced the best effects, and was pivotal for complete task interpretations. Redundant object information also improved the multimodal interpretation. Redundant number information had less effect, and proved difficult to interpret, particularly in determining the difference between target and destination information.

The simple integrator algorithm, using a weighted comparison of the inputs, and making use of task and content knowledge proved successful in creating better multimodal interpretations. Such a simple approach, exploiting redundant information, particularly in situations where individual input can be error prone, has shown that redundancy has made a significant contribution to a multimodal interpretation. 


\section{References}

Akamatsu, M.; Sato, S. (1994); A multi-modal mouse with tactile and force feedback. International Journal of Human-Computer Studies, Vol. 40, pp443453, 1994.

Adler, R.B.; Rodman, G. (1988); Understanding Human Communication; 3rd edition, 1988.

Alty, J.L. (1991); Multimedia - What is it and how do we exploit it? Proceedings of $H C I$ '91, pp31-44, 1991.

Alty, J.L.; Bergan, M.; Craufurd. P. (1993); Experiments using Multimedia Interfaces in Process Control: Some Initial Results. Computing and Graphics, Vol. 17, No. 3, pp 205-218, 1993.

Alty, J.L. (1998); Private Communication.

Ando, H.; Kikuchi, H.; Hataoka, N. (1995); Agent-typed Multimodal Interface Using Speech, Pointing Gestures and CG. Symbiosis of Human and Artifact; Anzai, Ogawa; Mori, eds.; pp29-34, 1995.

Ando, H.; Kitahara, Y.; Hataoka, N. (1994); Evaluation of Multimodal Interface using Spoken Language and Pointing Gesture on Interior Design System. International Conference on Spoken Language Processing, pp567-570, 1994.

Baecker, R.M.; Buxton, W.A.S. (1987); Readings in HCI - A Multidisciplinary approach, Chapter 8, pp357-365, 1987.

Baluja, S.; Pomerleau, D.A. (1994); Non-Intrusive Gaze Tracking Using Artificial Neural Networks. Advances in Neural Information Processing Systems (NIPS), Vol. 6; Cowan, J.D., Tesauro, G.; Alspector, J., eds., 1994. 
Basil, M.D. (1994); Multiple Resource Theory I: Application to Television Viewing. Communication Research, Vol. 21, No. 2, pp177-207, 1994.

Bellalem, N.; Romary, L. (1997); Structural Analysis of Co-verbal Deictic Gesture in Multimodal Dialogie Systems. Proceedings of Gesture Workshop 1996: Progress in Gestural Interaction, ppl41-153, Springer Verlag, 1997.

Bellik, Y (1997); Modality Integration: Speech and Gesture. Survey of the State of the Art in Human Language Technology; Cole, R, ed; Available from the Centre for Spoken Language Understanding, Oregon Graduate Institute, USA, 1997.

Bernsen, N.O. (1995); A taxonomy of Input Modalities available from Centre for Cognitive Informatics, Roskilde University, Roskilde, 1995.

Billinghurst, M.; Savage, J. (1996a); Adding Intelligence to the Interface. Proceedings of the IEEE 1996 Virtual Reality Annual International Symposium, pp 168-176, Piscataway, NJ, IEEE Press, 1996.

Billinghurst, M.; Savage, J.; Oppenheimer, P.; Edmond, C. (1996b); The Expert Surgical Assistant: An Intelligent Virtual Environment with Multimodal Input. Medicine meets Virtual Reality IV: Health Care in the Information Age, pp590607, Amsterdam, IOS Press, 1996.

Bodenhausen, U.; Manke, S. (1993a); Automatically Structured Neural Networks For Handwritten Character and Word Recognition. ICANN '93, 1993.

Bodenhausen, U.; Manke, S. (1993b); Connectionist Architectural Learning for High Performance Character and Speech Recognition. Proceedings of the 1993 International conference on Acoustics, Speech, and Signal Processing (ICASSP '93), 1993.

Bolt, R.A. (1980); "Put-that-there": Voice and Gesture at the Graphics Interface. Computer Graphics, Vol. 14, Part 3, pp262-270, 1980.

Bolt, R.A. (1984); The Human Interface - where people and computers meet, Chapter 3, pp35-52, 1984.

Bolt, R.A. (1987); Conversing with Computers. Readings in Human-Computer Interaction: A Multidisciplinary Approach, pp694-702, 1987.

Bordegoni, M. (1994); Parallel Use of Hand Gestures and Force-Input Device for Interacting with 3D and Virtual Reality Environments. International Journal of Human-Computer Interaction, Vol. 6, No. 4, pp391-413, 1994.

Bordegoni, M.; Faconti, G.P. (1996); Architectural Models of Gesture Systems. Proceedings of Gesture Workshop 1996: Progress in Gestural Interaction, pp6173, Springer Verlag, 1997. 
Bos, E.; Huls, C.; Claassen, W. (1994); EDWARD: full integration of language and action in a multimodal user interface. International Journal of HumanComputer Studies, Vol. 40, pp473-495, 1994.

Bourguet, M-L.; Ando, A. (1997); Speech Timing prediction in multimodal human-computer interaction. Proceedings of INTERACT '97, pp453-460, Chapman \& Hall, 1997.

Bratko, I. (1986); Programming for Artificial Intelligence. Addison-Wesley, London, 1986.

Bregler, C.; Hild, H.; Manke, S.; Waibel, A. (1993a); Improving Connected Letter Recognition by Lipreading. International Conference on Acoustics, Speech and Signal Processing (ICASSP ‘93), 1993.

Bregler, C.; Konig, Y. (1994); "Eigenlips" for Robust Speech Recognition. International Conference on Acoustics, Speech and Signal Processing (ICASSP '94), 1994.

Bregler, C.; Manke, S.; Hild, H.; Waibel, A. (1993b); Bimodal Sensor Integration on the Example of "Speech-Reading". Proceedings of the International Conference on Neural Networks, 1993.

Briggs, R.O.; Beck, B.S.; Dennis, A.R.; Carmel, E.; Nunamaker, J.F.; Pfarrer, R. (1992); Is the Pen Mightier Than the Keyboard?. Proceedings of the $25^{\text {th }}$ Hawaii International Conference on System Science, Vol. 3, pp. 201-210, 1992.

Brooks, F.P.; Ouh-Young, M.; Batter, J.J.; Kilpatrick, P.J. (1990); Project GROPE - Haptic Displays for Scientific Visualisation. Computer Graphics, Vol. 24, Number 4, pp177-185, 1990.

Buxton, William (1987); There's More To Interaction than meets the eye: Some Issues in Manual Input. Readings in Human-Computer Interaction: A Multidisciplinary Approach, pp366-375, 1987.

Caloini, Andrea; Tosolini, Paolo (1994); Time and gesture based interfaces a test case: Shinjuku Guide. UK Toolbook User Conference, 1994.

Cassell, J.; Steedman, M.; Badler, N.; Pelachaud, C.; Stone, M.; Douville, B.; Prevost, S.; Achorn, B. (1994); Modeling the Interaction between Speech and Gesture. Proceedings of the 16th Annual Conference of the Cognitive Science Society, Georgia Institute of Technology, Atlanta, USA, 1994.

Chatty, S.; Lecoanet, P. (1996); Pen Computing for Air Traffic Control. Proceedings of CHI 96, pp87-94.

Cherry, C. (1966); On Human Communication. The M.I.T. Press, Massachusetts, 1966. 
Cohen, P.R. (1992); The Role of Natural Language in a Multimodal Interface. Proceedings of the $5^{\text {th }}$ Annual Symposium on User Interface Software and Technology, pp143-149, 1992.

Coutaz, J.; Paterno, F.; Faconti, G.; Nigay, L.; Salber, D. (1993); A Comparison of Approaches for Specifying Multimodal Interactive Systems. Proceedings of ERCIM Workshop on Multimodal Human-Computer Interaction, pp165-174, 1993.

Dahlback, N.; Jonsson, A.; Ahrenberg, L. (1993); Wizard of Oz studies - why and how. Knowledge-Based Systems, Vol. 6, No. 4, December 1993.

Davis, J.; Shah, M. (1996); Gesture Recognition; Technical Report available from Department of Computer Science, University of Central Florida, Orlando, FL 32816, 1996.

Defresne, A.; Martial, O.; Ramstein, C. (1995); Multimodal User Interface System for Blind and "Visually Occupied" Users: Ergonomic Evaluation of the Haptic and Auditive Dimensions. Human-Computer Interaction, Proceedings of Interact'95, pp163-168, 1995.

DeHaemer, M.J.; Wright, G.; Dillon, T.W. (1994); Automated Speech Recognition for Spreadsheet Tasks: Performance Effects for Experts and Novices. International Journal of Human-Computer Interaction, Vol. 6, No. 3, pp299-318, 1994.

Duchnowski, P.; Hunke, M.; Busching, D.; Meier, U.; Waibel, A. (1995); Toward Movement-Invariant Automatic Lip-reading and Speech Recognition. Proceedings of the 1995 International Conference on Acoustics, Speech and Signal Processing, Vol. 1, pp109-12, 1995.

Duchnowski, P.; Meier, U.; Waibel, A. (1994); See me, Hear me: Integrating Automatic Speech Recognition and Lip-reading. ICSLP '94, International Conference on Spoken Language Processing, pp547-50, Vol. 2, 1994.

Eberts, R. (1994) User Interface Design, Prentice-Hall Inc., 1994.

Edwards, A.D.N. (1992); Redundancy and Adaptability; Multimedia Interface Design in Education; Edwards, A.D.N. \& Holland, S. (eds.); pp145-155, 1992.

Faconti, G.; Bordegoni, M.; Kansy, K.; Trahanias, P.; Rist, T.; Wilson, M. (1996); Formal Framework and Necessary Properties of the Fusion of Input Modes in User Interfaces. Interacting With Computers 8 (2), pp134-161, Elsevier, Amsterdam, July 1996.

Fishman, E.S.; Padilla, E. (1997); Speech Recognition Software Overview Summer/Fall 1997. Speech Recognition Update August 1997. Available from http://www.voicerecognition.com/article_8_97.html. 
Frohlich, D.M. (1991); The Design Space of Interfaces. Proceedings of the 1st Eurographics Workshop on Multimedia, Chapter 5, pp53-69, Kjelldahl, L. ed., 1991.

Geissler, J. (1995); Gedrics: The Next Generation of Icons. Human-Computer Interaction, Proceedings of Interact '95, pp73-78, 1995.

Hanne, K-H (1992); Multimodal Communication, Natural Language and Direct Manipulation (Gestures) in Human-Computer Interaction. Multimedia Interface Design in Education, pp157-175, 1992.

Hanne, K-H.; Bullinger, H-J. (1992); Multimodal Communication: Integrating Text and Gestures. Multimedia Interface Design. Blattner, Meera, M.; Dannenberg, Roger, B., eds.; ACM Press, pp. 127-138, 1992.

Hill, W.; Wroblewski, D.; McCandless, T.; Cohen, R. (1992); Architectural Qualities and Principles for Multimodal and Multimedia Interfaces. Multimedia Interface Design. Blattner, Meera, M.; Dannenberg, Roger, B., eds.; ACM Press, pp. 311-318, 1992.

Hinde, C.J.; Bray, A.D.; Herbert, P.J.; Launders, V.A.; Round, D. (1989); A Truth Maintenance Approach to Process Planning. Artificial Intelligence in Manufacturing, Proceedings of the $4^{\text {th }}$ International Conference on the Applications of Artificial Intelligence in Engineering, pp171-188, 1989.

Hummels, C.; Smets, G.; Overbeeke, K. (1997); An Intuitive Two-Handed Gestural Interface for Computer Supported Product Design. Gesture and Sign Language in Human-Computer Interaction. Wachsmuth, I.; Frohlich, M. (eds); International Gesture Workshop, Bielefeld, Germany, September 1997 Proceedings. Lecture Notes in Artificial Intelligence 1371, Springer, pp197-208, 1997.

Hunke, M.; Waibel, A. (1994); Face Locating and Tracking for HumanComputer Interaction. Proceedings of the $28^{\text {th }}$ Asimolar Conference on Signals, Systems and Computers, Vol. 2, pp1277-81, 1994.

Hutchins, E (1989); Metaphors for Interface Design. The Structure of Multimodal Dialogue; Taylor, M.M, Neel, F, Bouwhuis, D.G, eds.; pp11-28, 1989.

Iyanaga, S.; Kawada, Y. (eds); (1977) Encylopedic Dictionary of Mathematics. The MIT Press, Cambridge, 1977.

James, M. (1987); Pattern Recognition. BSP Professional Books, Oxford, 1987.

Kato, N.; Fukuda, N.; Nakagawa, M. (1995); An Experimental Study of Interfaces Exploiting a Pen's Merits. Symbiosis of Human and Artifact; Anzai, Ogawa, Mori, eds.; pp555-559, 1995. 
Keates, S.; Robinson, P. (1998); The use of gestures in multimodal input. Available from http://rehabwww.eng.cam.ac.uk/papers/lsk12/assets98/assetslsk.htm, 1998.

Kelley, A.J.; Higuschi, T.; Salcudean, S.E. (1995); An integrated haptographical user interface using a force feedback mouse. Symbiosis of Human and Artifact, pp713-718; Anzai, Ogawa, Mori, eds.; 1995.

Koons, D.B.; Sparrell, C.J.; Thorisson, K.R. (1993); Integrating Simultaneous Input from Speech, Gaze, and Hand Gestures. Intelligent Multimedia Interfaces, pp257-276, AAAI Press, 1993.

Kramer, A. (1997); Classifying Two Dimensional Gestures in Interactive Systems. Gesture and Sign-Language in Human-Computer Interaction. Proceedings of the International Gesture Workshop, Bielefeld, Germany, pp3748. Lecture Notes in Artificial Intelligence, 1371, 1997.

Kuijpers, Evert; Wilson, Michael (1992); A Multi-Modal Interface for Man Machine Interaction with Knowledge Based Systems-MMI. Proceedings of EastWest International Conference on Human Computer Interaction, Vol. 2, pp373$377,1992$.

Lai, J.; Vergo, J. (1997); MedSpeak: Report Creation with Continuous Speech Recognition. Proceedings of CHI 97, Atlanta, GA, USA, pp431-438, 1997.

Lang, A. (1995); Defining Audio/Video Redundancy from a Limited-Capacity Information Processing Perspective. Communication Research, Vol. 22, No. 1, February 1995, pp. 86-115.

Lu, S.; Yoshizaka, S.; Kamiya, T.; Miyai, H. (1995); A Human-Computer Dialogue Agent with Body Gestures, Hand Motion, and Speech. Symbiosis of Human and Artifact; Anzai, Ogawa, Mori, eds.; pp17-22, 1995.

Mack, R.; Lang, K. (1989); A Benchmark Comparison of Mouse and Touch Interface Techniques for an Intelligent Workstation Windowing Environment. Proceedings of the Human Factors Society $33^{\text {rd }}$ Annual Meeting, pp325-329, 1989.

MacKenzie, I.S.; Nonnecke, B.; Riddersma, S. (1994); Alphanumeric entry on pen-based computers. International Journal of Human-Computer Studies, Vol. 41, pp775-792, 1994.

MacKenzie, I.S.; Oniszczak, A. (1998); A Comparison of Three Selection Techniques for TouchPads; Proceedings of CHI '98, pp336-343, 1998.

Mahach, K.R (1989); A Comparison of Computer Input Devices: Linus Pen, Mouse, Cursor Keys and Keyboard. Proceedings of the Human Factors Society $33^{\text {rd }}$ Annual Meeting, pp330-334, 1989. 
Maloof, M.A.; Michalski, R.S. (1995); Learning Symbolic Descriptions of 2D Shapes for Object Recognition in X-Ray Images. Proceedings of the 8th International Symposium on Artificial Intelligence, Monterrey, Mexico, October 17-20, pp124-131, 1995.

Maloof, M.A.; Michalski,R.S. (1997); Learning Symbolic Descriptions of Shape for Object Recognition in X-Ray Images. Expert Systems with Applications, Vol. 12, No. 1, pp.11-20, 1997.

Manke, S.; Bodenhausen, U. (1994); A Connectionist Recognizer for On-line Cursive Handwriting Recognition. International Conference on Acoustics, Speech and Signal Processing (ICASSP, ‘94), 1994.

Manke, S.; Finke, M.; Waibel, A. (1995a); Npen+t: A Writer Independent, Large Vocabulary On-Line Cursive Handwriting Recognition System. Proceedings of the International Conference on Document Analysis and Recognition, 1995.

Manke, S.; Finke, M.; Waibel, A. (1995b); The Use of Dynamic Writing Information in a Connectionist On-line Cursive Handwriting Recognition System. Advances in Neural Information Processing Systems, Vol. 7; Tesauro, G., Touretsky, D.; Alspector, J., eds.; 1995.

Marmollin, H. (1991); Multimedia from the Perspectives of Psychology. Multimedia, Systems, Interaction and Applications, $1^{\text {st }}$ Eurographics Workshop, Stockholm, Sweden, pp39-52, 1991.

Masui, T. (1998); An Efficient Text Input Method for Pen-based Computers; Proceedings of CHI '98, pp328-335, 1998.

Mayes, J.T. (1992); The 'M-Word': Multimedia Interfaces and Their Role in Interactive Learning Systems. Multimedia Interface Design in Education; pp 122, Edwards, A.D.N. \& Holland, S. (eds.), Springer Verlag, 1992.

McNeill, D.; Duncan, S. (1996); Language as gesture (gesture as language). Proceedings of Workshop on the Integration of Gesture in Language and Speech (WIGLS), Delaware, 1996. Available from http://www.asel.udel.edu/WIGLS

Mertz, C.P.; Lecoanet, P. (1997); GRIGRI: Gesture Recognition on Interactive Graphical Radar Image. Progress in Gestural Interaction. Proceedings of Gesture Workshop, York, 1996, pp187-198. Springer Verlag, 1997.

Moore, David S. (1995); The Basic Practice of Statistics. W.H. Freeman \& Co., USA, 1995.

Moran, D.B.; Cheyer, A.J.; Julia, L.E.; Martin, D.L.; Park, S. (1998); Multimodal user interfaces in the Open Agent Architecture. Knowledge-Based Systems, 10 , pp295-303, 1998. 
Nakagawa, S.; Zhang, J.; Chengcharoen, W. (1995); A multi-modal Interface with Speech and Touch Screen. Symbiosis of Human and Artifact, pp213-218, Anzai; Ogawa; Mori, eds.; 1995.

Newby, G.B. (1993); Gesture Recognition Using Statistical Similarity. Proceedings of the Virtual Reality and Persons with Disabilities Conference, 1993.

Nickerson, R.S. (1987); On Conversational Interaction with Computers. Readings in Human-Computer Interaction: A Multidisciplinary Approach, pp681-693, 1987.

Nigay, L.; Coutaz, J. (1993); A Design Space for Multimodal Systems: Concurrent Processing and Data Fusion. InterCHI '93, pp172-178, 1993.

Norman, D.A. (1986); Cognitive Engineering. User Centered System Design, Norman \& Draper, eds., Chapter 3, pp31-65, Lawrence Erlbaum Associates, 1986.

Norusis, Marija. J. (1993); SPSS for Windows Base System User's Guide Release 6.0, SPSS Inc., USA, 1993.

Ohashi, T.; Yamanouchi, T.; Matsunaga, A.; Ejima, T. (1995); Multimodal Interface with Speech and Motion of Stick: CoSMoS. Symbiosis of Human and Artifact, 1995.

Oviatt, S. (1995) Predicting Spoken disfluencies in human-computer interaction. Computer Speech and Language, 9, pp19-35, 1995.

Oviatt, S. (1996); Multimodal Interfaces for Dynamic Interactive Maps. Proceedings of CHI 96, pp95-102, 13-18 April, 1996.

Oviatt, S.; DeAngeli, A.; Kuhn, K. (1997); Integration and Synchronization of Input Modes during Multimodal Human-Computer Interaction. $\mathrm{CHI} 97,22-27$ March, pp.415-422, 1997.

Oviatt, S.; Olsen, E. (1994); Integration Themes in Multimodal Human-Computer Interaction. Proceedings of International Conference on Spoken Language Processing, Vol. 2, pp551-554, 1994.

Oviatt, S.; VanGent, R. (1996); Error resolution During Multimodal HumanComputer Interaction. Proceedings of International Conference on Spoken Language Processing, 1996.

Perkins, K.; Socket Wrapper code; Department of Computer Studies, Loughborough University, 1997. http://www-staff.lboro.ac.uk/ cokapl .

Quinlan, J. Ross (1993); C4.5: Programs for Machine Learning, Morgan Kaufmann Publishers, San Mateo, California, 1993. 
Ramstein, C. (1995); A Multimodal User Interface System with Force Feedback and Physical Models. Human-Computer Interaction, Proceedings of Interact '95, pp157-162, 1995.

Rich, E; Knight, K (1991); Artificial Intelligence, McGraw-Hill, 1991.

Richardson, M. (1966); Fundamentals of Mathematics. The Macmillan Company, New York, 1966.

Robbe, S.; Carbonell, N.; Dauchy, P. (1997); Constrained vs spontaneous speech and gestures for interacting with computers: A comparitive empirical study. Proceedings of INTERACT`97, pp445-452, Chapman \& Hall, 1997.

Rubine, D. (1991) Specifying Gestures by Example. Computer Graphics 25, 4, pp 329-337; ACM Press, New York, 1991.

Rudnicky, A.I.; Hauptmann, A.G. (1992); Multimodal Interaction in Speech Systems. Multimedia Interface Design. Blattner, Meera, M.; Dannenberg, Roger, B., eds.; ACM Press, pp. 147-171, 1992.

Schank, R. (1972); Conceptual Dependency: A theory of natural language understanding. Cognitive Psychology 3, pp552-631, 1972.

Schiele, B.; Waibel, A. (1995); Gaze Tracking Based on Face-Color. Proceedings of the International Workshop on Automatic Face and Gesture Recognition, 1995.

Shannon, C.E.; (1951); Prediction and Entropy of Printed English. Bell System Technology Journal, 30, pp50-64, 1951.

Sharman, R.A. (1994); Speech Recognition in the Office: How the technology Supports Dictation. The Computer Journal, Vol. 37, No. 9, pp735-744, 1994.

Shneiderman, B. (1982) The future of interactive systems and the emergence of direct manipulation. Behaviour and Information Technology, 1, pp237-256, 1982.

Shneiderman, B. (1998); Designing the User Interface: Strategies for Effective Human-Computer Interaction. Third Edition, Addison-Wesley, 1998.

Sidhu, Charanjit K.; Coyle, Gerry (1995); Delivering the promise of speech interfaces. Symbiosis of Human and Artifact, pp529-533; eds. Anzai; Ogawa; Mori, 1995, Elsevier Science.

Sinha, A.K.; Network Programming in Windows NT. Addison-Wesley Publishing Company, 1996.

Smith, A; Dunaway, J.; Demasco, P.; Peischl, D. (1996); Multimodal Input for Computer Access and Augmentative Communication. Proceedings of ASSETS ‘96, Vancouver, British Columbia, Canada, 1996, pp80-85, ACM. 
Tou, J.T.; Gonzalez, R.C. (1974); Pattern Recognition Principles. AddisonWesley Publishing Company, 1974.

Tubbs, S.L.; Moss, S. (1994); Human Communication. McGraw-Hill, Inc. 1994.

Vetere, F. (1997); Redundancy in Multimedia Systems. Proceedings of INTERACT ‘97, pp648-650, Chapman \& Hall, 1997.

Vetere, F.; Howard, S.; Leung, Y. (1997); A Multimedia Interaction Space. Proceedings of INTERACT '97, pp, Chapman \& Hall, 1997.

Vo, M.T.; Houghton, R.; Yang, J.; Bub, U.; Meier, U.; Waibel, A.; Duchnowski, P. (1995); Multimodal Learning Interfaces. Proceedings of the ARPA Spoken Language Technology Workshop, 1995.

Vo, M.T.; Waibel, A. (1993a); A Multimodal Human-Computer Interface: Combination of Speech and Gesture Recognition. Adjunct Proceedings of InterCHI'93, 1993.

Vo, M.T.; Waibel, A. (1993b); Multimodal Human-Computer Interaction. Proceedings of ISSD '93, 1993.

Vo, M.T.; Waibel, A. (1996); Multimodal Human-Computer Interaction. Draft available from Alex Waibel at School of Computer Science, Carnegie Mellon University, Pittsburgh, PA 15213-3890, U.S.A, 1996.

Vo, M.T.; Wood, C. (1996); Building an application framework for speech and pen input integration in multimodal learning interfaces. Proceedings of Acoustic, Speech and Signal Processing Conference, Atlanta, GA, pp3545-3548, May 1996.

Wachsmuth, I.; Frohlich, M. (eds) (1997); Gesture and Sign Language in Human-Computer Interaction. International Gesture Workshop, Bielefeld, Germany, September 1997 Proceedings. Lecture Notes in Artificial Intelligence 1371, Springer, 1997, pp23-158,209-258.

Waibel, A.; Suhm, B.; Vo, M.T.; Yang, J. (1997); Multimodal Interfaces for Multimedia Information Agents. Proceedings of Acoustics, Speech and Signal Processing IEEE Conference, Munich, 1997, pp167-170.

Waibel, A.; Vo, M.T.; Duchnowski, P.; Manke, S. (1994); Multimodal Interfaces. AAAI Review Journal, Special Issue, 1994.

Weimer, D.; Ganapathy, S.K. (1992); Interaction Techniques using Hand Tracking and Speech Recognition. Multimedia Interface Design. Blattner, Meera, M.; Dannenberg, Roger, B., eds.; ACM Press, pp. 109-126, 1992.

Wolf, C.G. (1992); A Comparitive Study of Gestural, Keyboard, and Mouse Interfaces. Behaviour and Information Technology, Vol. 11, No. 1, pp13-23, 1992. 
Wolf, C.G.; Kassler, M.; Zadrozny, W.; Opyrck, L. (1997); Talking to the Conversation Machine: An Empirical Study. Proceedings of INTERACT '97, pp461-468, Chapman \& Hall, 1997.

Wolf, Catherine G.; Koved, Larry; Kunzinger, Edward (1995); Ubiquitous Mail: Speech and Graphical User Interfaces to an Integrated Voice/E-Mailbox. HumanComputer Interaction, Proceedings of Interact '95, pp247-252, 1995.

Wolf, C.G.; Morrel-Samuels, P. (1987); The use of hand-drawn gestures for text editing. International Journal of Man-Machine Studies, Vol. 27, pp91-102, 1987.

Wolf, C.G.; Zadrozny, W. (1998); Evolution of the Conversation Machine: A Case Study of Bringing Advanced Technology to the Marketplace; Proceedings of $C H I$ '98, pp488-495, 1998.

Yang, J.; Stiefelhagen, R.; Meier, U.; Waibel, A. (1998); Visual Tracking for Multimodal Human Computer Interaction; Proceedings of CHI '98, pp140-147, 1998.

Zemany, P; DeCegama, A; Smith, J (1993); Fusion of Radar and Acoustic Data. Proceedings of the 1993 International Symposium on Intelligent Control, pp279$284,1993$.

Zhao, R.; Kaufmann, H.J.; Kern, T.; Muller, W. (1995); Pen-based Interfaces in Engineering Environments. Symbiosis of Human and Artifact, pp531-536; eds. Anzai; Ogawa; Mori, 1995, Elsevier Science.

-; 3com website; http:/www.palm.com/products/input/index.html, 1998.

-; DragonVoiceTools Programmer's Guide, DragonDictate Software, February 1995, vt1.1. 
Appendices 


\section{Appendix A}

Supplemental Tables and Figures 
Error rate data from Section 5.6.1.1, Figure 18.

\begin{tabular}{lllllllllll}
\hline User \# & 1 & 2 & 3 & 4 & 5 & 6 & 7 & 8 & 9 & 10 \\
\hline $\begin{array}{l}\text { Predicted decision } \\
\text { tree error rates }\end{array}$ & 13.2 & 15.2 & 18 & 21.6 & 12.2 & 19.3 & 15.3 & 8.8 & 11.7 & 16 \\
$\begin{array}{l}\text { Actual decision } \\
\text { tree error rates }\end{array}$ & 49.5 & 43.6 & 26.7 & 27.8 & 44.3 & 37.2 & 34.3 & 45.6 & 45.1 & 35.4 \\
$\begin{array}{l}\text { Actual production } \\
\text { rules error rates }\end{array}$ & 49.5 & 43.3 & 27.4 & 27.8 & 44.4 & 33.9 & 35.4 & 46.3 & 45 & 36.6 \\
\hline
\end{tabular}

Table 24: Predicted decision tree, actual decision tree $\mathcal{\&}$ actual production rule error rates per subject, Experiment 1, Chapter 5.

Speech, Gesture and Multimodal Data from the main 30 subject experiment (Section 8.4, pp161)

\begin{tabular}{|c|c|c|c|c|}
\hline $\begin{array}{l}\text { Subject } \\
\text { number }\end{array}$ & Accent & speech & gesture & multimodal \\
\hline 1 & British & 60.4811 & 57.68374 & 66.36971 \\
\hline 2 & Other & 80.41237 & 67.96537 & 77.92208 \\
\hline 3 & British & 73.77049 & 71.11597 & 79.43107 \\
\hline 4 & British & 64.18919 & 63.61656 & 76.03486 \\
\hline 5 & British & 60.88435 & 71.55172 & 74.35345 \\
\hline 6 & British & 65.58442 & 68.79121 & 72.74725 \\
\hline 7 & Other & 69.18033 & 80.47722 & 84.16486 \\
\hline 8 & British & 71.57191 & 77.32181 & 84.44924 \\
\hline 9 & Other & 79.65517 & 54.34783 & 68.69565 \\
\hline 10 & Other & 74.65753 & 81.4978 & 88.10573 \\
\hline 11 & Other & 67.86885 & 67.69231 & 76.7033 \\
\hline 12 & Other & 55.0173 & 81.22271 & 79.0393 \\
\hline 13 & British & 78.47682 & 74.18655 & 82.4295 \\
\hline 14 & British & 66.77741 & 73.1441 & 81.00437 \\
\hline 15 & British & 74.49664 & 79.91266 & 83.18777 \\
\hline 16 & British & 69.62457 & 77.23214 & 88.83929 \\
\hline 17 & British & 69.67742 & 81.59645 & 89.80044 \\
\hline 18 & British & 75.1634 & 79.51542 & 90.30837 \\
\hline 19 & British & 64.50512 & 77.27273 & 78.13853 \\
\hline 20 & British & 64.90066 & 76.44444 & 84.44444 \\
\hline 21 & British & 79.38144 & 74.83444 & 84.10596 \\
\hline 22 & British & 70.13889 & 58.68132 & 72.30769 \\
\hline 23 & British & 63.7931 & 66.23094 & 69.28105 \\
\hline 24 & British & 66.44518 & 65.58442 & 74.67532 \\
\hline 25 & British & 80.47945 & 79.47598 & 89.30131 \\
\hline 26 & British & 69.83051 & 73.71938 & 79.95546 \\
\hline 27 & British & 69.17808 & 75.21739 & 83.69565 \\
\hline 28 & British & 74.17219 & 65.28384 & 74.45415 \\
\hline 29 & British & 74.74403 & 77.77778 & 86.66667 \\
\hline 30 & Other & 71.66124 & 82.75862 & 90.30172 \\
\hline Averages & & 70.22 & 72.74 & 80.36381 \\
\hline
\end{tabular}

Table 25: Speech, Gesture and Multimodal Recognition Success rates 


\begin{tabular}{lccc}
\hline & Spect & Cestire & Novitinodial \\
\hline cut & 97.27 & 0.00 & 73.96 \\
copy & 95.41 & 54.20 & 87.97 \\
paste & 94.33 & 15.02 & 86.96 \\
moveV & 75.96 & 45.82 & 61.35 \\
moveH & 77.31 & 68.37 & 77.56 \\
moveP & 77.00 & 67.24 & 77.26 \\
moveN & 74.56 & 52.44 & 64.47 \\
heart & 51.73 & 80.68 & 84.31 \\
flower & 91.60 & 80.34 & 83.25 \\
fire & 93.71 & 77.87 & 87.10 \\
ear & 75.64 & 79.03 & 85.96 \\
earth & 71.62 & 78.56 & 87.03 \\
flowerpot & 92.81 & 75.77 & 87.12 \\
tick & 94.25 & 73.61 & 88.15 \\
\hline averages - & 84.55 & 43.3 & 75.65 \\
command & & & 86.13 \\
\hline averages - & 81.62 & 77.98 & \\
objects & & & \\
\hline
\end{tabular}

Table 26: Recognition rates of command and objects for speech, gesture and multimodal 


\begin{tabular}{|c|c|c|c|c|c|c|}
\hline & Speech & Gesture & Multimodal & Speech & Gesture & Multimodal \\
\hline 1 & 67.19 & 96.17 & 94.54 & 39.64 & 66.67 & 70.48 \\
\hline 2 & 67.65 & 93.84 & 91.94 & 56.06 & 66.67 & 73.87 \\
\hline 3 & 53.19 & 92.57 & 82.18 & 38.67 & 68.22 & 69.16 \\
\hline 4 & 73.04 & 92.90 & 91.72 & 26.28 & 71.29 & 73.27 \\
\hline 5 & 67.54 & 84.24 & 87.50 & 39.39 & 76.84 & 79.79 \\
\hline 6 & 65.73 & 95.88 & 93.30 & 43.40 & 72.64 & 69.81 \\
\hline 7 & 69.57 & 99.42 & 91.28 & 64.00 & 62.34 & 66.23 \\
\hline 8 & 73.50 & 91.33 & 89.60 & 40.85 & 65.12 & 68.60 \\
\hline 9 & 70.73 & 93.47 & 90.45 & 50.00 & 70.59 & 70.59 \\
\hline 10 & 67.77 & 86.80 & 83.25 & 51.39 & 71.76 & 76.47 \\
\hline 11 & 66.36 & 95.54 & 87.90 & 44.07 & 68.89 & 64.44 \\
\hline 12 & 77.68 & 97.73 & 94.32 & 46.88 & 68.24 & 72.94 \\
\hline 13 & 60.34 & 98.35 & 92.31 & 43.53 & 67.68 & 71.72 \\
\hline 14 & 60.42 & 99.24 & 95.42 & 54.72 & 73.42 & 73.42 \\
\hline 15 & 74.65 & 92.56 & 90.08 & 54.41 & 71.76 & 74.12 \\
\hline 16 & 65.35 & 96.40 & 88.49 & 37.86 & 78.65 & 79.78 \\
\hline 17 & 57.89 & 100.00 & 97.40 & 43.84 & 58.67 & 61.33 \\
\hline 18 & 64.38 & 97.50 & 92.50 & 47.62 & 68.57 & 67.14 \\
\hline 19 & 76.92 & 99.15 & 94.02 & 41.51 & 71.43 & 72.86 \\
\hline 20 & 62.65 & 94.07 & 98.31 & 41.18 & 73.81 & 75.00 \\
\hline 21 & 69.86 & 95.45 & 92.73 & 44.09 & 76.04 & 81.25 \\
\hline 22 & 56.04 & 99.08 & 93.58 & 30.30 & 75.34 & 79.45 \\
\hline 23 & 69.12 & 96.72 & 88.52 & 43.75 & 75.90 & 79.52 \\
\hline 24 & 63.51 & 93.81 & 87.63 & 64.71 & 70.73 & 69.51 \\
\hline 25 & 72.09 & 96.10 & 93.51 & 45.90 & 66.67 & 72.00 \\
\hline averages & 66.93 & 95.13 & 91.30 & 45.36 & 70.32 & 72.51 \\
\hline
\end{tabular}

Table 27: Recognition rates of target and destination numbers for speech, gesture and multimodal 


\begin{tabular}{|c|c|c|c|c|c|c|c|c|c|c|c|c|}
\hline (1.0.1.4. & Speech and & gesture mode & sagree & 34 & Speech and g & gesture modes & disagree & (4) & Information & from only & mode availa & ble v. ? \\
\hline $\begin{array}{l}\text { Subject } \\
\text { aumber }\end{array}$ & commands & objects & targets & destinafions & commands & objects & targets. & destinations & commands & fojects & targets & destinations \\
\hline 1 & 100 & 97.36842 & 100 & 100 & 87.23404 & 45 & 21.05263 & 30.76923 & 36.84211 & 90.56604 & 97.2973 & 77.77778 \\
\hline 2 & 85.18519 & 100 & 100 & 83.33333 & 92.85714 & 84.61538 & 70 & 57.14286 & 58.97436 & 87.5 & 87.30159 & 70.4918 \\
\hline 3 & 86.11111 & 100 & 100 & 94.73684 & 85 & 84.61538 & 53.33333 & 34.78261 & 41.46341 & 90 & 88.52459 & 70.58824 \\
\hline 4 & 75.86207 & 100 & 100 & 91.66667 & 78.43137 & 73.68421 & 53.84615 & 33.33333 & 50 & 86.95652 & 85.9375 & 69.76744 \\
\hline 5 & 97.56098 & 100 & 100 & 100 & 82.85714 & 93.33333 & 47.05882 & 33.33333 & 60 & 91.48936 & 84.05797 & 76.19048 \\
\hline 6 & 78.78788 & 98 & 100 & 0 & 90.38462 & 94.11765 & 41.66667 & 0 & 50 & 87.03704 & 96.34146 & 85.71429 \\
\hline 7 & 81.81818 & 100 & 100 & 100 & 75.75758 & 75 & 27.27273 & 17.64706 & 32.43243 & 95.71429 & 98.57143 & 78 \\
\hline 8 & 100 & 100 & 100 & 90 & 46.37681 & 81.81818 & 12.5 & 21.42857 & 35.89744 & 86.95652 & 97.36842 & 81.08108 \\
\hline 9 & $89 . \overline{47368}$ & 97.82609 & 100 & 60 & 71.875 & 52.17391 & 45.45455 & 47.05882 & 37.83784 & 93.47826 & 92.20779 & 66.66667 \\
\hline 10 & 97.4359 & 98.33333 & 100 & 68.75 & 82.6087 & 66.66667 & 16.66667 & 35 & 55.26316 & 95.83333 & 98.50746 & 74.35897 \\
\hline 11 & 90.56604 & 100 & 100 & 100 & 85.71429 & 53.33333 & 57.14286 & 35.71429 & 62.5 & 95.65217 & 91.42857 & 85.18519 \\
\hline 12 & 86.95652 & 100 & 100 & 76.19048 & 100 & 85.71429 & 40 & 20 & 70.73171 & 95.83333 & 89.0625 & 84.21053 \\
\hline 13 & 72.22222 & 100 & 100 & 100 & 80 & 87.5 & 41.66667 & 61.53846 & 27.90698 & 92.59259 & 96.8254 & 93.75 \\
\hline 14 & 94.44444 & 91.89189 & 94.11765 & 42.85714 & 86.66667 & 87.5 & 100 & 30.76923 & 76.74419 & 88.88889 & 95.2381 & 43.75 \\
\hline 15 & 96.36364 & 100 & 100 & 85.18519 & 89.18919 & 90.90909 & 33.33333 & 30.76923 & 71.42857 & 85.71429 & 95.45455 & 69.23077 \\
\hline
\end{tabular}

Table 28: Percentage of correct multimodal interpretations when speech and gesture agreed, disagreed and when only one mode available, subjects 1-15 
Appendix A

\begin{tabular}{|c|c|c|c|c|c|c|c|c|c|c|c|c|}
\hline \multirow{2}{*}{$\begin{array}{l}\text { Subject } \\
\text { Dumber }\end{array}$} & \multicolumn{4}{|c|}{ Speech and gesture modes agree } & \multicolumn{4}{|c|}{ Speech and gesture modes disagree } & \multicolumn{4}{|c|}{ Information from only one mode available } \\
\hline & commands & objects & targets & destinations & Commands & objects & targets & destinations & conmmands & objects & targets & destinations \\
\hline 16 & 100 & 100 & 100 & 100 & 100 & 72 & 57.89474 & 62.5 & 55.71429 & 92 & 84.50704 & 78.94737 \\
\hline 17 & 95.91837 & 100 & 100 & 63.63636 & 85.18519 & 100 & 31.57895 & 22.22222 & 73.91304 & 85.33333 & 92.30769 & 68.51852 \\
\hline 18 & 89.13043 & 100 & 100 & 100 & 76.92308 & 84.61538 & 54.54545 & 42.85714 & 60.60606 & 91.11111 & 95.08197 & 82.92683 \\
\hline 19 & 92.30769 & 100 & 100 & 76.92308 & 90.69767 & 35.29412 & 66.66667 & 33.33333 & 50 & 94.52055 & 97.26027 & 79.54545 \\
\hline 20 & 100 & 97.95918 & 100 & 86.66667 & 62.7451 & 45.45455 & 57.14286 & 9.090909 & 78.43137 & 98.27586 & 92.1875 & 75 \\
\hline 21 & 71.42857 & 100 & 100 & 71.42857 & 74 & 100 & 77.27273 & 45 & 30 & 95.45455 & 84.05797 & 83.33333 \\
\hline 22 & 97.87234 & 100 & 100 & 56.52174 & 93.75 & 100 & 57.14286 & 0 & 71.42857 & 98.03922 & 91.5493 & 61.53846 \\
\hline 23 & 89.28571 & 100 & 100 & 80.95238 & 100 & 90.90909 & 62.5 & 20 & 75.67568 & 95.74468 & 98.46154 & 63.07692 \\
\hline 24 & 82.05128 & 100 & 100 & 100 & 79.41176 & 100 & 64.70588 & 61.90476 & 25.64103 & 98.18182 & 90.27778 & 75.75758 \\
\hline 25 & 98.33333 & 100 & 100 & 94.11765 & 90.90909 & 91.66667 & 100 & 33.33333 & 79.31034 & 93.47826 & 98.59155 & 74.07407 \\
\hline 26 & 100 & 100 & 100 & 71.42857 & 96.15385 & 75 & 59.09091 & 40.625 & 63.29114 & 89.79592 & 86.53846 & 75.67568 \\
\hline 27 & 100 & 100 & 100 & 88.88889 & 69.44444 & 68.75 & 27.77778 & 30 & 71.18644 & 91.37931 & 100 & 74.4186 \\
\hline 28 & 95.34884 & 100 & 100 & 72.41379 & 97.14286 & 78.94737 & 69.23077 & 28.57143 & 66.66667 & 89.79592 & 93.22034 & 64 \\
\hline 29 & 95.65217 & 95.74468 & 100 & 54.54545 & 83.78378 & 72.72727 & 100 & 60 & 75 & 86.48649 & 98.66667 & 75 \\
\hline 30 & 94.73684 & 100 & 100 & 88 & 87.09677 & 92.85714 & 71.42857 & 42.85714 & 74.28571 & 97.91667 & 98.33333 & 74.57627 \\
\hline ए.:. & Averages & 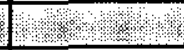 & \begin{tabular}{|l|l|}
$.3 \%$ \\
\end{tabular} & (2.3. & (6. & (2.8. & 1.:- & H. & (3. & 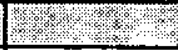 & (1) & 1. \\
\hline (30 subjects) & 91.16178 & 99.23745 & 99.80392 & 79.94143 & 84.0732 & 78.80677 & 53.93242 & 34.05274 & 57.30575 & 92.05754 & 93.1722 & 74.43841 \\
\hline
\end{tabular}

Table 29: Percentage of correct multimodal interpretations when speech and gesture agreed, disagreed and when only one mode available, subjects $16-30$ 


\section{Appendix B}

Experimental guides 


\section{Gesture Study}

The game involves moving coloured shapes around a grid by making gestures on a touchscreen. Gestures should be made with the touchpen supplied.

You will be asked to make around 130 gestures which should take around 10-15 minutes. Instructions will appear on screen. If you make a mistake the system will wait for you to repeat the gesture.

\section{Gestures:}

PASTE $=\checkmark$ TICK (the object for pasting will be on the clipboard automatically)

\section{CUT $=x$ CROSS}

MOVE $=$ ( ( LINES

\section{SELECT $=$ O CIRCLE}

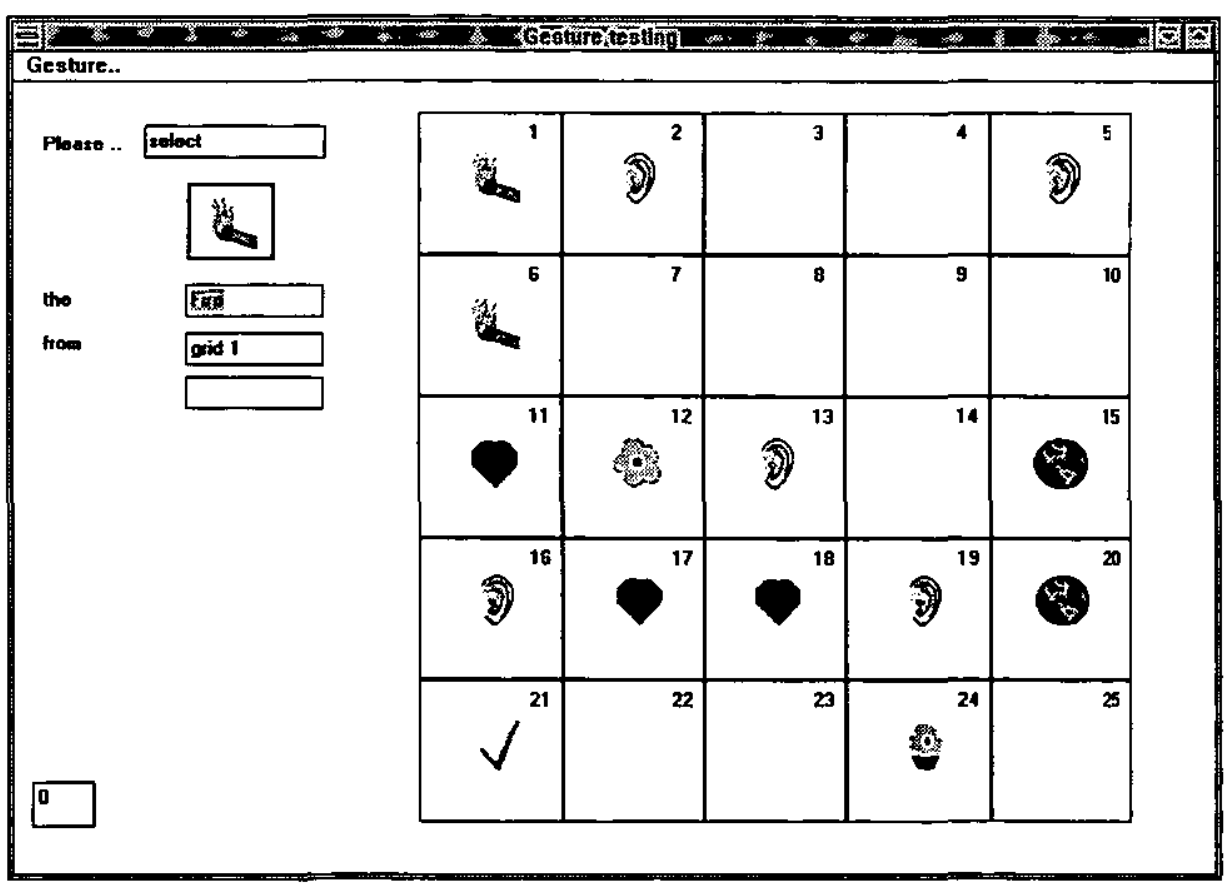

Thank you for your help! 


\section{Speech and Gesture Study}

This experiment asks you to move coloured shapes around a grid by following on-screen speech and gesture requests. Speech is made into the microphone provided, gestures made on the touchscreen with the touchpen supplied.

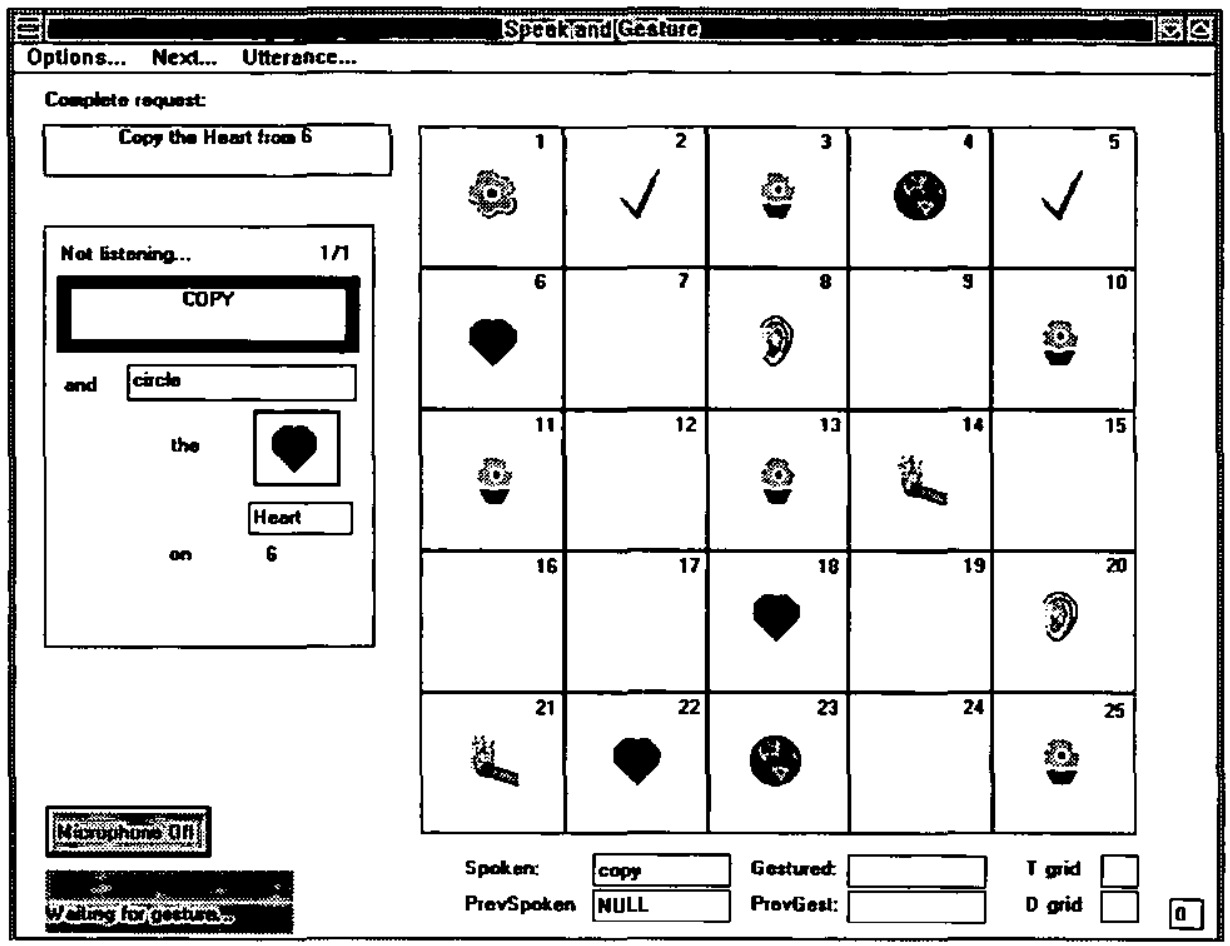

\section{Format}

First of all you will be asked to speak some words to provide a personal vocabulary file. This will take around 5 minutes.

You will be asked to do around 130 speech and gesture combinations, speaking a word or phrase followed by a gesture. This will be split into two sessions which should take around 10-15 minutes each. You can have a break in between the sessions.

In each speech and gesture combination the speech is always performed before the gesture. A small information window in the bottom left-hand corner of the screen shows whether the system is waiting for speech or waiting for gesture.

\section{Geroptone Of}

Wating for speech 


\section{Speech Requests}

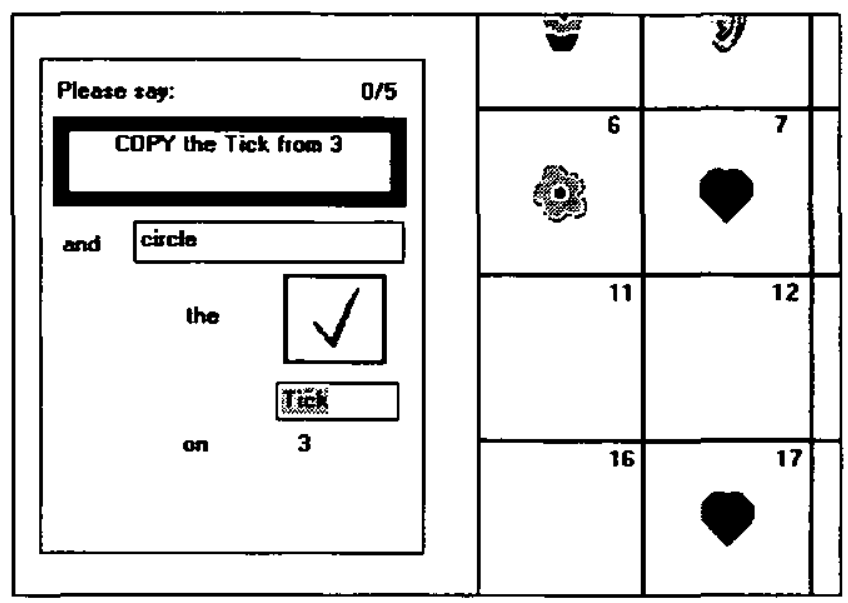

Speech requests appear on screen in a black-edged text box. e.g. the speech request is "COPY the Tick from 3" in the figure above. When speaking, it is necessary to pause between words slightly. The word count shown above the textbox will increase when a word has been received. Please wait for the word count to increase before speaking the next word.

\section{Gesture Requests}

Gesture requests appear below speech requests. Please perform each gesture firmly and in one complete motion (i.e. a break anywhere in a gesture is not allowed).

Cross gestures are made up of two separate line gestures. Please wait for the busy pointer to disappear before completing the second part of a cross gesture.

The necessary gestures and their command meaning are as follows.

\section{$\boldsymbol{x}_{\text {CROSS }}=\mathrm{CUT} \quad \mathrm{O}$ CIRCLE $=$ COPY $(\quad$ LINES $=$ MOVE}

TICK = PASTE (the object for pasting will be on the clipboard automatically)

Thank you for your time! 


\section{Appendix C}

Papers

McKenzie Mills, K.; Alty, J.L. (1996); Using rule induction in two-dimensional gesture recognition. Department of Computer Studies internal report, 1996.

McKenzie Mills, K.; Alty, J.L. (1997); Investigating the Role of Redundancy. Gesture and Sign-Language in Human-Computer Interaction. Proceedings of the International Gesture Workshop, Bielefeld, Germany, pp 159-171. Lecture Notes in Artificial Intelligence, 1371, 1997.

McKenzie Mills, K.; Alty, J.L. (1998); Integrating speech and two-dimensional gesture input - a study of redundancy between modes. Proceedings of $\mathrm{OzCHI}$ 1998, University of South Australia, Adelaide, South Australia; Calder, P., \& Thomas, B. (eds.), IEEE Computer Society, California, pp6-13,1998. 


\title{
Using Rule Induction in Two Dimensional Gesture Recognition
}

\author{
Karen McKenzie Mills \& James L. Alty \\ Department of Computer Studies, Loughborough University, Loughborough, UK. \\ \{K.C.M.Mills, J.L.Alty\}@lboro.ac.uk
}

\begin{abstract}
Two dimensional gesture recognition has many applications from handwriting recognition to air traffic control systems. Most methods of interpretation rely on statistical tools using parametric models to describe gesture sets for future recognition. This paper describes the use of rule induction as a method of interpreting raw two-dimensional gesture data. The simple two-dimensional gesture experimentation is described and the results from the use of rule induction on the experimental input is examined. The final classification models produced from rule induction indicate recognition rates of $80 \%$ on unseen gesture data.
\end{abstract}

\section{Introduction}

Our long term aim is to create a successful, relatively inexpensive, input recognition system which allows human beings to communicate in a natural way with a computer by using simple gestures in parallel with voice commands. Unfortunately, simple techniques for recognising either voice commands or gestures have high error rates, so research workers have progressively used more sophisticated techniques in attempts to remedy this problem (for example, in the case of gesture, data gloves or 3-D vision have been used). Whilst these approaches, using extensive computing resources, are more successful, they are unlikely to produce solutions which can be used in everyday applications, at least not in the foreseeable future. We have therefore re-examined the way human beings solve the problem in order to formulate possible alternative approaches. It is our contention that the human approach works well because of three important characteristics:

1. Human beings processes the two inputs (voice and gesture) in parallel, using either input channel to assist the other at times of confusion.

2. Human Beings have an implicit understanding of the likely errors in different channels and use this understanding to weight input from different channels.

3. Human beings rely heavily on knowledge about the content of likely messages, in the task context, to assist in disambiguation. They instinctively use gesture to support speech when ambiguity is possible or when they perceive that understanding may be compromised.

By exploiting these characteristics, we hope to achieve our goal. We are therefore developing two simple, independent input systems for recognising gesture and voice input, which will inevitably be error prone. We will then process these combined inputs using knowledge about the nature of likely errors on each channel together with elementary task knowledge, to achieve acceptable levels of disambiguation. It is hoped that, as in the human case, the whole will be more 
powerful that the separate parts.

This paper concentrates mainly upon our work on the gesture aspects of the system. In our simple system (and the system is intended to be deliberately simple), a gesture is made on a 2-dimensional touchscreen. Many approaches to two-dimensional gesture recognition currently use statistical methods to process the recognition data. For example, Newby [4] used statistical similarity, the sum of squares approach, while Rubine [8] used a linear classifier on a set of gesture features which had been extracted earlier. Statistical, Parametric and Linear Classification Models are well suited to optimising gesture recognition given high quality input data. However, our aim is not optimisation, rather we wish to identify critical attributes which recur in many gestures and which are often partial descriptions only. We require our analysis to not only provide characterisations for successful recognition, but also characterise failure as well.

Statistical approaches are not well suited for our approach. An example of this is a Linear Classified based on the concept that the class can be expressed as a linear combination of the attribute values in some way. Then when training cases are supplied the optimum linear combination of attributes that best fits is searched so that the class can be decided [7]. This parametric approach is not desirable for our work as we require a more flexible approach than the linear classifier allows.

An alternative approach is Rule induction. Although research in rule induction has applied the technique for learning two-dimensional shapes in X-ray image applications [1] it does not appear to have been applied to simple twodimensional gesture recognition. It is suited to classification tasks which have the following properties: attribute-value description; predefined classes; discrete classes; sufficient data; or logical classification models. [7]. It is suited to attributes which can be expressed as discrete or numeric.

Attribute-value description: The data used for analysis should be expressible as a set of fixed attributes or properties. The properties should not differ from case to case, where a case in our approach is one example of a dataset from one individual gesture.

Predefined classes: The classes into which cases can be grouped have already been established. This is called supervised learning in the field of machine learning. Our classes are the predefined acceptable gestures.

Discrete classes: Classes must be distinct from one another i.e. mutually exclusive. A member of one class cannot be a member of another. There must also be considerably more cases than classes. This applies to our data. Gestures are distinct, and there will be many example gesture attempts.

Sufficient data: As induction looks for patterns in data, it follows that there must be enough cases for robust patterns to be identified. The number of attributes and classes, as well as the complexity of a classification model, all affect the amount of data required. 
Logical classification models: The output from this particular induction software produces a logical rather than arithmetic model of classification. In our case this is preferred to Arithmetic which calculate a class based on weights of attributes

At the start of our investigation we reviewed the available inexpensive induction software. The ID3 and CN2 systems [1],[5],[6] were considered but could not cope sufficiently with detailed numerical data such as real valued attributes which were a key feature of our gesture data. The more recent development of the C4.5 machine learning program proved suitable for our purpose [7].

\section{Experimentation}

\subsection{First Dataset}

The application involved the manipulation of coloured shapes around a numbered grid, using gesture only (Figure 2). A session lasted approximately eight minutes, and involved the creation of sixty gestures. Subjects were asked to select shapes by circling them, paste shapes by ticking them, delete shapes using a cross,or move shapes from one grid square to another using horizontal, vertical or sloped lines.

An initial two-dimensional gesture experiment was carried out with ten subjects. Subjects followed on-screen requests, but no feedback was given on success or failure. All subjects were computer literate but most were not familiar with using gesture as an input method.

On-screen requests were of the form

Please perform action on object from grid $X$ to grid $Y$

e.g. Please SELECT the HEART from grid 1

Please MOVE the EAR from grid 22 to grid 8 etc.

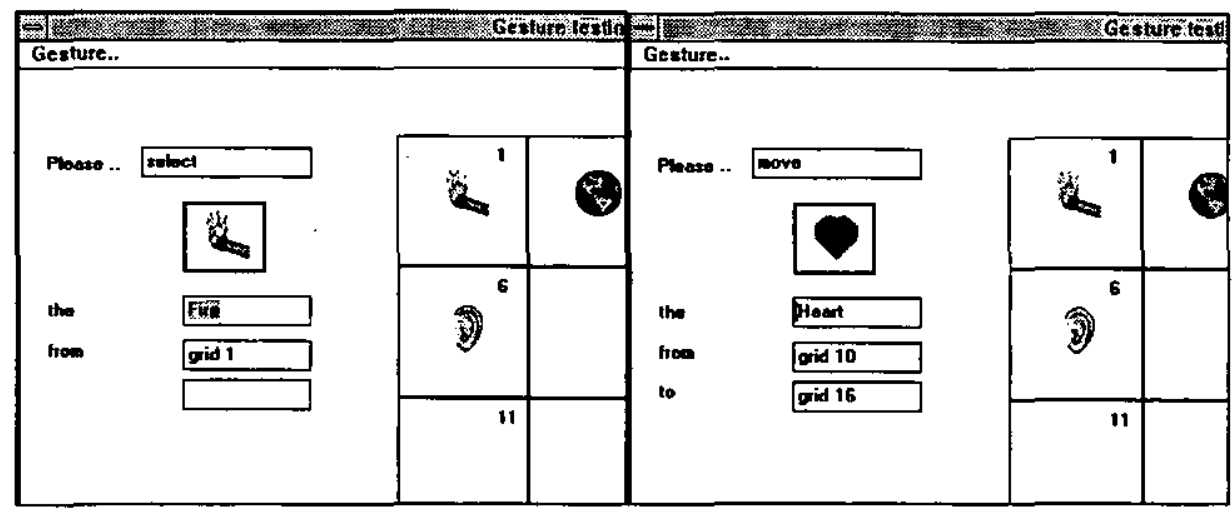

Figure 1: On screen request examples

Users were asked to create gestures in two sessions producing a set of 120 gestures per user. Analysis of the data is discussed in Section 2.1.1. 


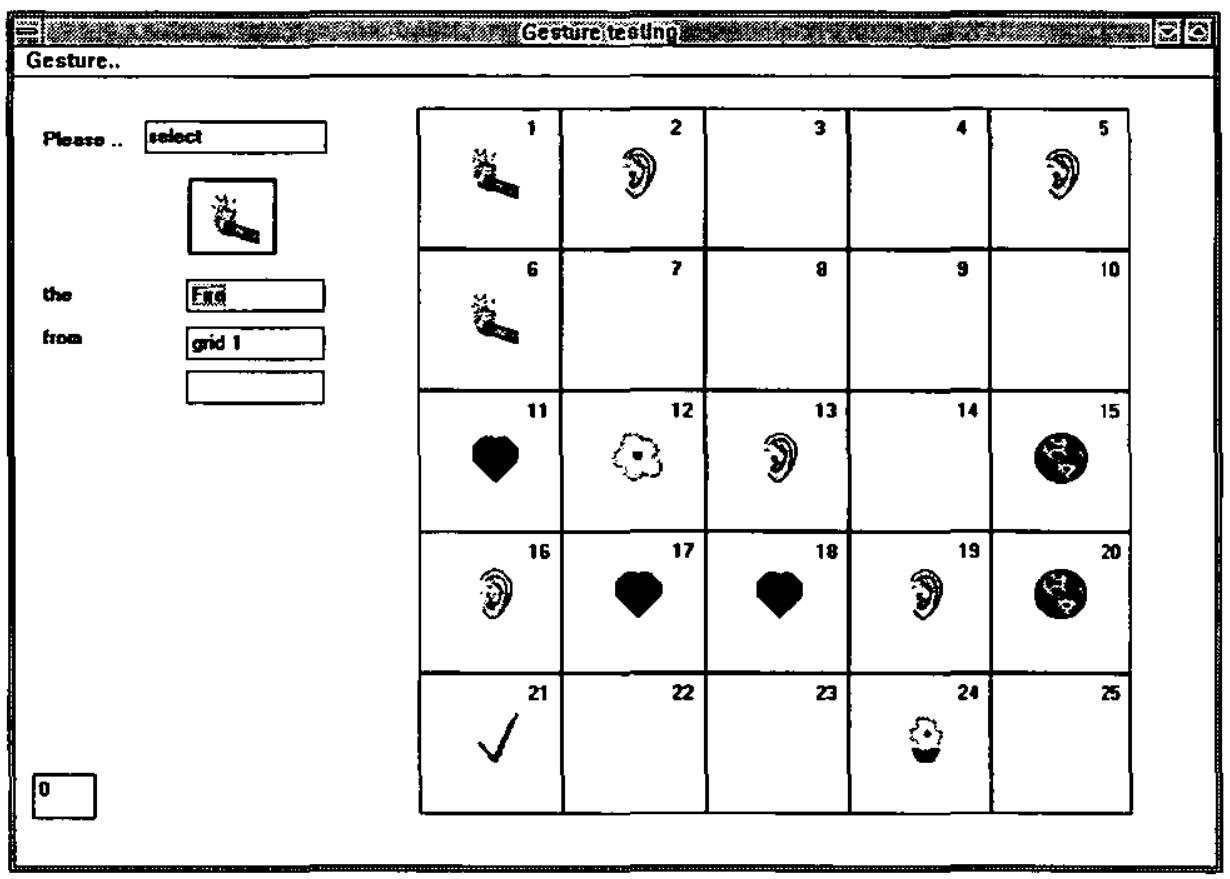

Figure 2: Screenshot of the gesture only experiment

\subsubsection{Results}

The data gained from these initial gesture only experiments was analysed using the C4.5 induction software [7]. Each individual's data was processed as training data to give a set of decision trees and production rules. Associated confidence percentages for the efficiency of the trees and rules in classifying further unseen data was also produced. It was then possible to test these trees and rules on data from all the other users in the group to gain an understanding of how generalised they were in classifying unseen gesture data.

Several factors were observed to effect the trees and rules produced: number of classes; inclusion of a cross class in the data; complete inclusion or exclusion of the cross data. The cross is actually a two component gesture, comprising a line of negative gradient and a line of positive gradient, in some order, which intersect one another. However, in the defined task a cross differs from a move or paste gestures (which involve negative or positive gradients) since crosses are only performed within a grid rather than across grids (as is the case with move gestures). Therefore discrete classes to group the component cross gestures were included.

The analysis of the data gave the following information:

- decision trees and production rules

- predicted error rate

- number of times the rule was used to classify cases

- number of cases which satisfied a rule but were of the wrong class

- confusion matrix to show where mis-classifications occurred

A sample of the information provided from one user follows. 
Sample production rules generated from processing a decision tree

Rule 17:

horizontal \& $>21.875$

negative gradient $8>7.692$

positive gradient $8>6.25$

total points $>36$

$\rightarrow$ class circle $[88.28]$
Rule 14:

$\begin{array}{lcccr}\text { horizontal } & \& & > & & 5.714 \\ \text { horizontal } & \& & \& & & 21.875 \\ \text { negative } & \text { gradient } \& & > & 7.692 \\ \text { positive } & \text { gradient } 8 & > & 22.5 \\ \text { total } & \text { points } & > & 20 \\ \text {-> class tick }[90.68] & & \end{array}$

As seen above, the production rules consist of a set of attributes with tests that must be fulfilled to lead to the given class conclusion. The percentage figure shown after the class is an estimate by the system of how successful the rule will be in classifying future unseen cases.

\subsubsection{Error rates}

The decision tree analysis gives overall predicted error rates using the training data and also gave actual error rates when applied to test data. The production rule analysis of the data gives various rules and results, with a variety of predicted classification rates for individual rules.

Obvious errors were removed from the data before induction, such as gestures which clearly differed from the requested gesture. For some individuals, this reduced the dataset by half and the final dataset contained 923 cases rather than the initial 1200 cases gathered. Varying predicted error rates were obviously found between different users. The complete data set of 923 cases from all ten users was also induced to give overall rules.

The predicted error rates could not give an insight to the possible performance of the decision trees in recognising gestures as they often differed greatly from the actual error rates when using test data. The predicted error rates were produced by the induction software based only on the training data supplied at the time of compilation. These represent the prediction of the percentage of unseen cases which the decision tree would expect to classify correctly. These figures are more an opinion of the system than a tangible guide [7]. This is evidenced in the fact that the predicted error rates are fairly low, with overall classification confidences all greater than $78 \%$ (Figure 3). Clearly, only unseen cases analysed under the produced trees and rules give a guide to their actual efficiency.

Therefore, to gain figures on how accurate and useful each set of rules could be, each ruleset was tested on a dataset of unseen cases: the data from all the other users in the group excluding the subject whose trees and rules were currently in use. A plot of the decision tree predicted error rate, the decision tree actual error rate and the production rule error rate follows. 


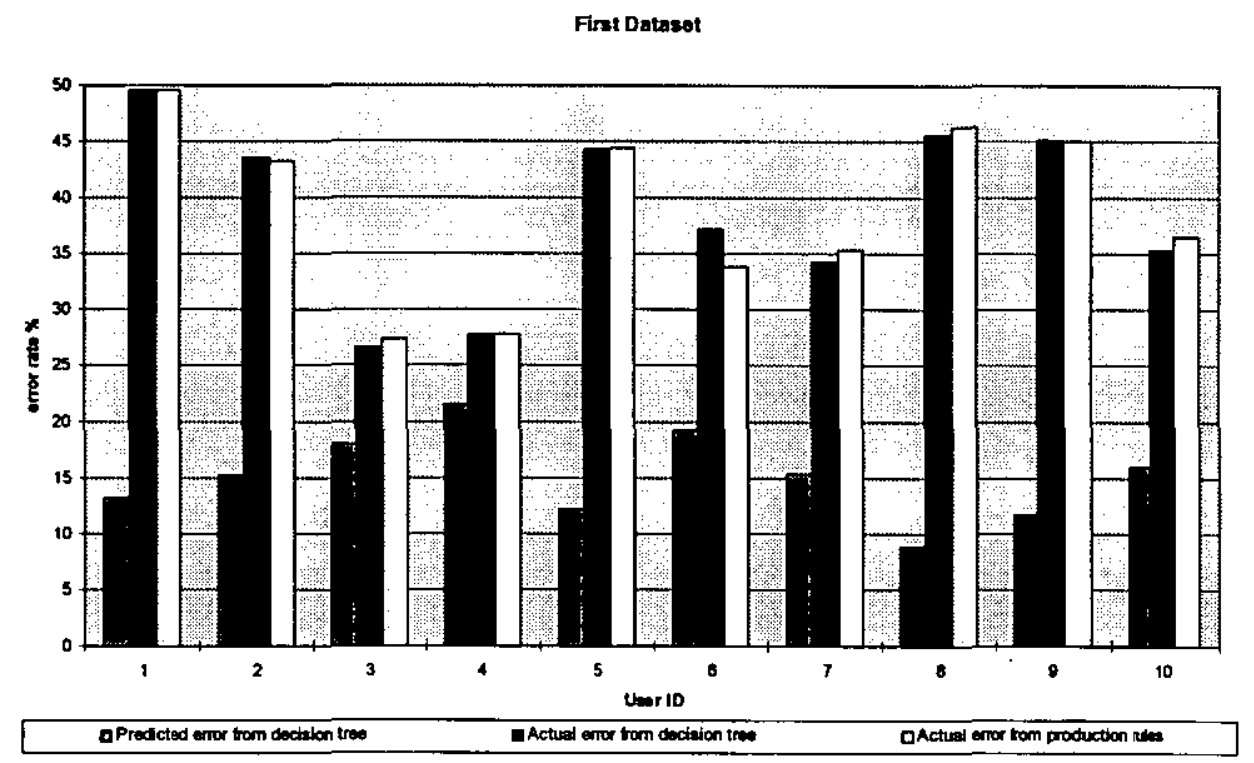

Figure 3: Error rates per user from decision tree and production rule models, 1st dataset

Therefore, each tree and ruleset was used to analyse a large dataset of between 800-923 cases. This gave more higher (and more realistic) error rates than the predictions suggested. There were some unexpected results. For instance, the rules of User 8 who had the lowest predicted error rate of $8.8 \%$, when tested against the dataset of all the users produced a high error rate of $45.6 \%$ and $46.3 \%$ (actual tree and actual rule error rates). This indicates that although the classification was successful in interpreting that individual's data, that user's data did not represent a set of typical gestures. Their trees and rules were therefore unhelpful when classifying other datasets.

An overall predicted error rate for the production rules was not available. Instead, predicted error rates were produced for each individual rule. In most cases the actual error rate was significantly higher than the predicted error rate when unseen cases were tested using the rules. On average, only one production rule, from the complete set of rules, would give an actual error rate lower than predicted.

\subsubsection{Interpretation}

Analysis and comparison of individual rules was difficult. In some cases there were no rules found to classify a particular gesture in the first dataset. This could be attributed to the imbalance of gestures in the first dataset. As this first experiment requested random move actions, there was not a controlled number of line gestures. Thus, for instance in one dataset no positive line gestures were requested of the user and so obviously no patterns were found. Therefore, the classification models produced from the first set of experimentation could be categorised as inefficient and fuzzy because of these problems.

These initial experiments showed that improvements would be required before 
obtaining a new dataset. Firstly, the new system needed to take account of observed errors made by users and request that they repeat such gestures. For instance, errors where users gestured in the wrong grids to those requested could result in the wrong gesture being made. Also, the data needed to be balanced over all gestures to ensure relevant rules could be deduced about each gesture. Previously, the data was governed by the number of actions performed instead of the number of gestures.

Also, another set of gesture only experimentation would give another dataset for induction using the trees and rules from the first experiment. Similarly the trees and rules from the second set of experimentation could be used on the first dataset to gauge their efficiency.

\subsection{Second Dataset}

\subsubsection{Error rates}

Another gesture-only experiment was then conducted with these improvements. This gave a larger dataset with none of the previous errors. Another ten users were asked to perform a set of 128 gestures using a similar format to the first experiment. This produced a dataset containing 1280 cases with all 7 possible gestures represented in equal numbers in the data. Each set of production rules gave rules for all the classes (gestures).

The actual error rates for the decision tree and production rules are plotted with the predicted error rate from the decision tree below.

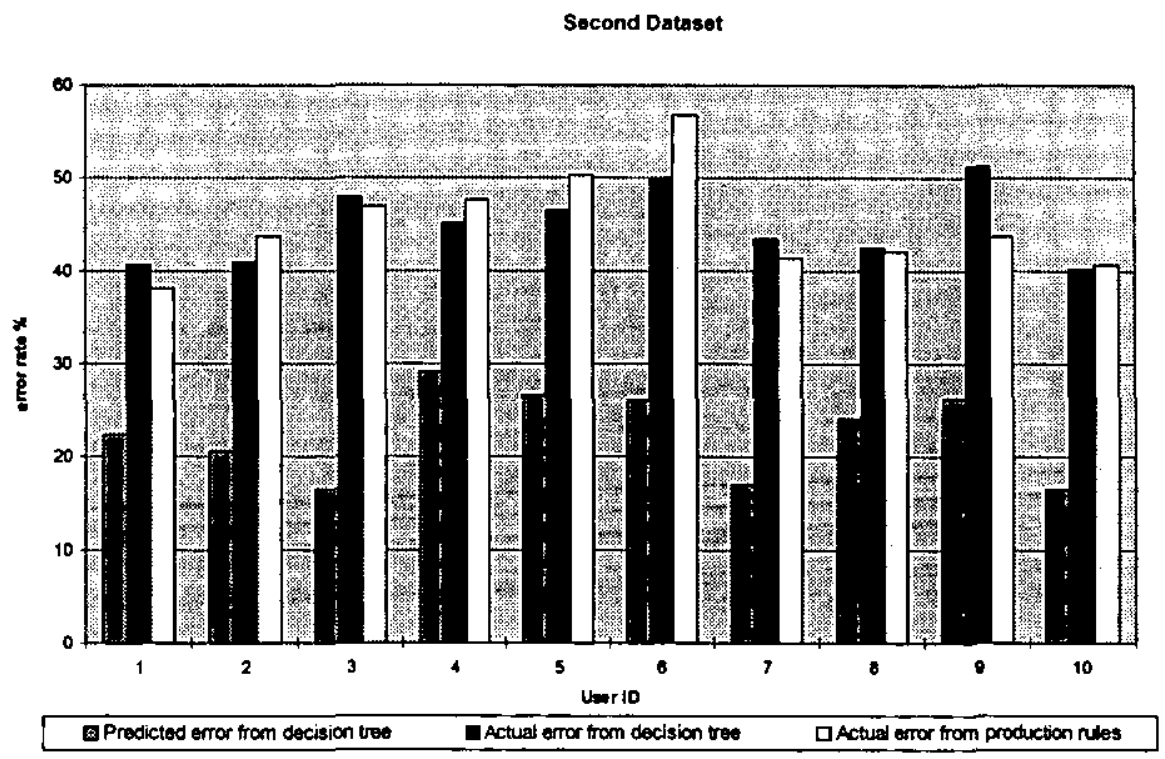

Figure 4: Error rates per user from decision tree and production rule models, 2nd dataset

The second dataset produced higher error rates on average than the first dataset. As described in the previous section predicted error rates were made for each 
individual production rule. As in the first dataset, in most cases the actual error rate was significantly higher than the predicted error rate when unseen cases were tested using the rules. On average, one quarter of the production rules gave an actual error rate lower than predicted for the second dataset.

The averages for the second dataset are plotted beside the averages from the first dataset in the following graph.

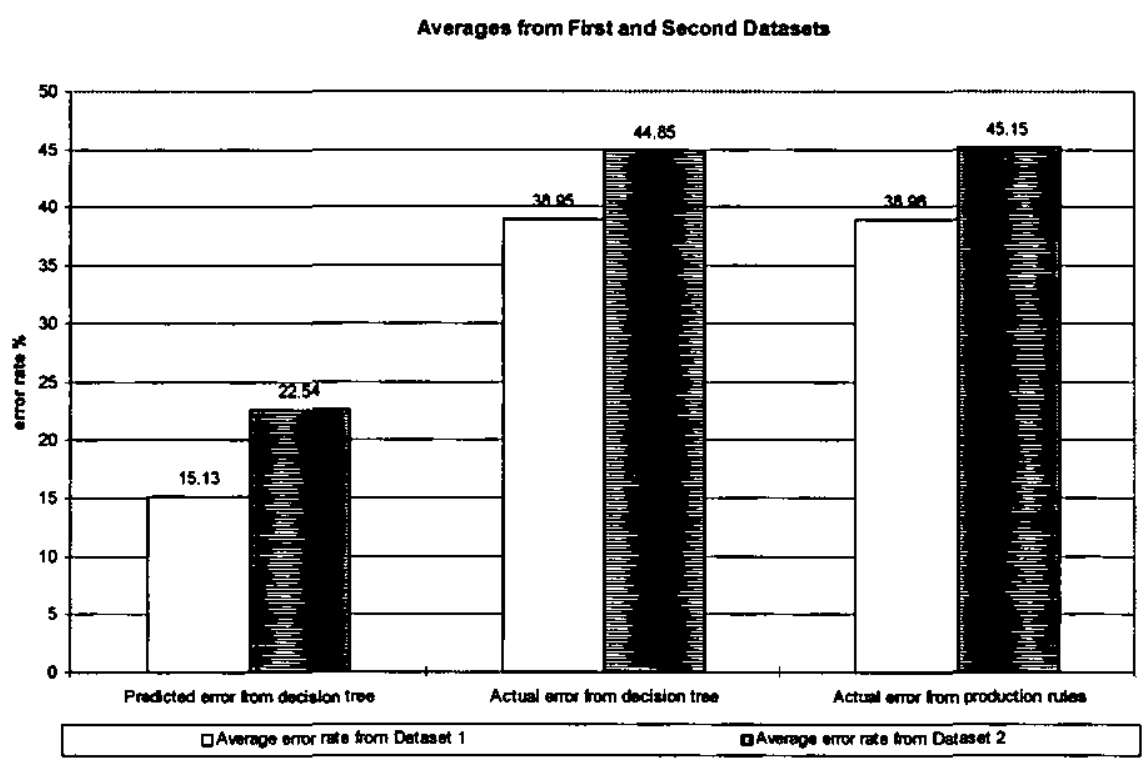

Figure 5: Average error rate for first and second datasets

The higher average error rates could be attributed to the fact that the second dataset is a more realistic representation of results. While the average error rate is higher, the standard deviation of production rule error rates is lower than that of the actual decision tree error rates (Figure 4).

The standard deviations for the actual error rates from Figure 4 are:

\begin{tabular}{|l|l|l|}
\hline error rate & $\begin{array}{l}\text { standard } \\
\text { deviation - 1st set }\end{array}$ & $\begin{array}{l}\text { standard deviation } \\
\text { - 2nd set }\end{array}$ \\
\hline actual decision tree & 7.45 & 3.79 \\
\hline actual production rule & 7.43 & 5.17 \\
\hline
\end{tabular}

This shows that the second dataset has produced a more balanced classification model than the first.

\section{Grouping Trees, Rules and Data}

The data from the first experiment was given as unseen test data to the decision trees and production rules generated from the second dataset (Case 1). Similarly, the data from the second experiment was given as unseen data to the decision trees and production rules generated from the first dataset (Case 2). The first dataset was smaller than the second due to the removal of errors. Therefore the size of dataset 1 was 923 cases, while dataset 2 was 1280 cases. Thus, column 1 
results are made with the number of test cases less than the number of training cases, while column 2 results are the opposite, the number of test cases exceed the number of training cases.

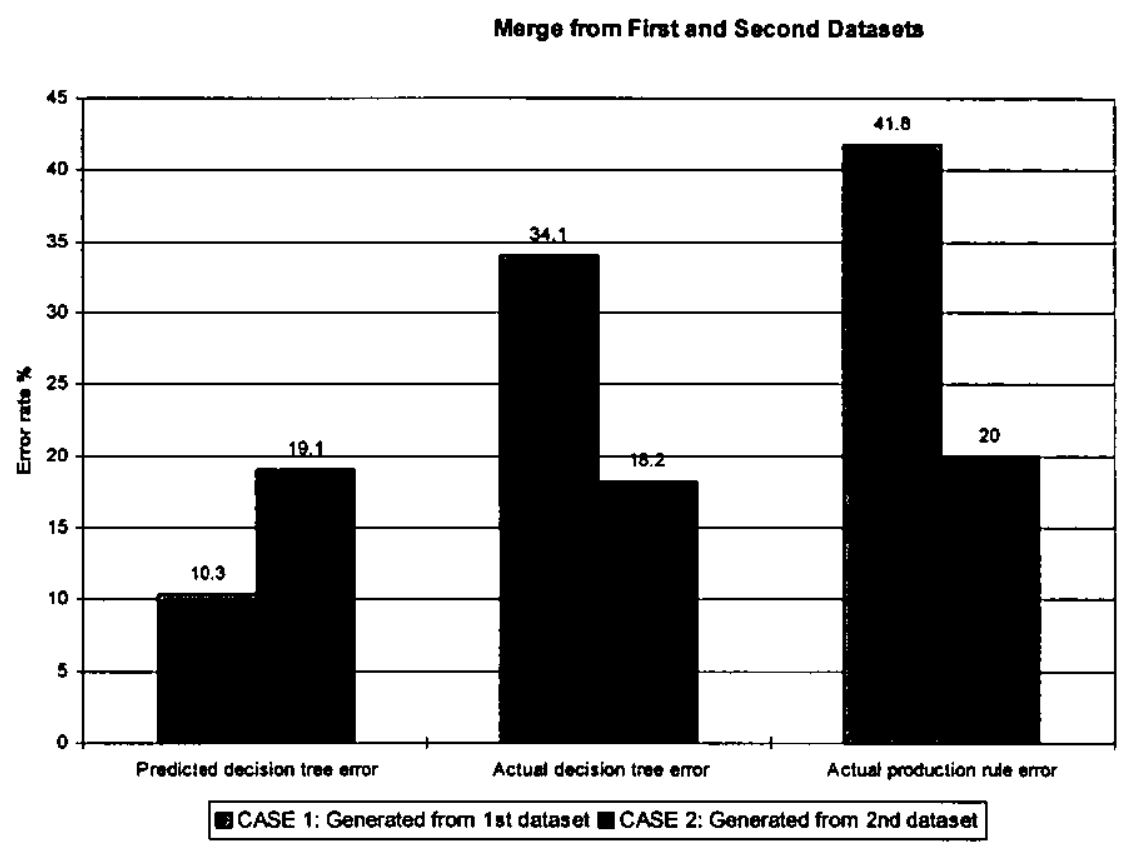

Figure 6: Error rates from the merge of all data

column 1: \# of training cases > \# of test cases, column 2: \# of training cases <\# of test cases

From Figure 6 we see a lower error rate from both decision tree and production rules for the first dataset used as test cases. This could be attributed to case 1 having a greater number of training cases than test cases, which affects the classification model produced [7]. With a larger training set it could be argued that a more robust pattern classification was possible.

Further analysis showed an increase in the error rate when subsets of the training data were used to induce new trees and rules. This could be expected given the results shown in Figure 6 . However, some unexpected results were revealed. When the set of training cases for case 1 was reduced by one user, i.e. by 128 cases, the error rate fell. Similarly when another user's data was removed, again the error rate fell. Only when another user's data was removed and the number of training cases fell below the number of test cases did the error rate rise again. This could perhaps be attributed to inefficient nodes and rules being removed in the smaller training set examples. It is acknowledged that many factors can affect the error rates when using induction [7]. Therefore, these results fit the overall expected behaviour of inducing differing sizes of datasets.

Using the merged data gave lower error rates than any of the individual or average error rates, making the merged trees and rules the preferred choice to use for the gesture recognition analysis. The decision tree gave lower error rates than 
the production rules. However, as the size of the decision tree and included sub trees amounted to twelve pages and proved difficult to interpret, the production rules were chosen instead. The difference in error rates between the methods was not large enough $(1.8 \%)$ to be of concern.

\section{Conclusions}

The preliminary results presented here show that recognition rates of around $80 \%$ can be expected when using rule induction as a method of interpreting twodimensional gesture data.

\section{References}

[1] Clark, P.; Niblett, T.; The CN2 Induction Algorithm IN Machine Learning 3, 4, pp261-284, 1989.

[2] Maloof, M.A. and Michalski, R.S.; Learning Symbolic Descriptions of 2D Shapes for Object Recognition in X-ray images IN Proceedings of the 8th International Symposium on Artificial Intelligence, Monterrey, Mexico, October 17-20, 1995.

[3] Mills, K.C.M. and Alty, J.L.; The Role of Redundancy in Multimodal Input Systems. Proceedings of Gesture Workshop 1997, Bielefeld, Germany. Springer Verlag 1997.

[4] Newby, G.B.; Gesture Recognition Using Statistical Similarity IN Proceedings of the Virtual Reality and Persons with Disabilities Conference, 1993.

[5] Quinlan, J.R.; Learning efficient classification procedures IN Machine learning: an artificial intelligence approach; Michalski, R.S.; Carbonell, J.G.; Mitchell, T,M,. (eds.).; Palo Alto, CA, 1983.

[6] Quinlan, J.R.; Induction of Decision Trees IN Machine Learning 1, 1, pp81-106, 1996.

[7] Quinlan, J. R., C4.5: Programs for Machine Learning, Morgan Kaufmann Publishers, San Mateo, California, 1993.

[8] Rubine, D., Specifying Gestures by Example IN Computer Graphics 25, 4, pp 329-337; ACM Press, New York, 1991.

[9] Michalski, R.S. various work at George Mason University, US. 


\title{
Investigating the Role of Redundancy in Multimodal Input Systems
}

\author{
Karen McKenzie Mills \& James L. Alty \\ Department of Computer Studies, Loughborough University, Loughborough, UK. \\ \{K.C.M.Mills, J.L.Alty\}@lboro.ac.uk
}

\begin{abstract}
A major concem of Human Computer Interaction is to improve communication between people and computer applications. One possible way of improving such communication is to capitalise on the way human beings use speech and gesture in a complementary manner, exploiting the redundancy of information between these modes. Redundant data input via multiple modalities, give considerable scope for the resolution of error and ambiguity. The research detailed in this paper describes implementation of a simple, inexpensive tri-modal input system accepting touch, two dimensional gesture and speech input. Currently the speech and gesture recognition systems operate separately. Truth maintenance and blackboard system architectures in a multimodal interpreter are proposed for the handling the integration between modes and task knowledge. Preliminary results from the two dimensional gesture recognition system are presented. Rule Induction is used for analysis of the two dimensional gesture data and preliminary classification results are presented. Current implementations and future work for the analysis of the role of redundancy are also discussed.
\end{abstract}

\section{Introduction}

In human to human communication, multiple channels are used, often combined in parallel. The communicative power of Speech, for example, is often enhanced through the use of gestures. Such gestures involve an extensive number of body movements (movement of eyes, limbs; body language etc.). Frequently, channel content is complementary, that is, the channels provide different information [20]. However, they also can contain redundant information. Humans, therefore, constantly 'send' a mixture of complementary and redundant information and, as a result, achieve a high success rate in communicating intentions to one another. The redundancy is particularly important where one or other of the channels becomes indistinct or noisy. In contrast, current computer input techniques are almost never used in parallel and are rarely redundant.

The concept of multiple channel human-computer interaction is not new. As far back as 1980 the 'Put-that-there' study at MIT [6] described a speech and 3D gesture input system. However, speech and gesture input have yet to be fully integrated into popular interfaces. Currently we only really use the mouse and keyboard, which are limited as they do not exploit all of the communication skills that human beings possess. 
Recently, efforts have been made to improve single mode input methods. Research in voice recognition has advanced to the stage where discrete voice input can be incorporated into multimodal interfaces [16] but because of the high cost of such approaches, discrete voice recognition is still not common in popular interfaces. A very recent advance is the availability of commercial continuous voice recognition systems [13]. Gesture recognition, covering two and three dimensions, ranging from two dimensional gesturing on screen to whole body movement is still in its infancy [5],[8],[18]. Research into the integration and combination of multiple modes is fairly new and little progress has been made in making the concept commonly available on our desktops, despite a large variety of initial studies reported between 1993 and 1997 [1],[2],[3],[7],[11],[12].

In multimodal systems, where input can be achieved through multiple channels, there are multiple and parallel sources of information. Initial studies into the integration of modalities has focused on combining modes in some way but has lacked a focus on how to combine modes and has not considered the benefits or why they occur [1],[2],[15],[17]. Later work has shown some evidence to suggest benefits in multimodal systems which incorporate several input modalities [17],[19],[26]. Scope for error resolution has been observed [19],[22] as has scope for the resolution of ambiguities and contradictions in the input [17],[26].

Although researchers are agreed that multiple inputs have benefits over single input, there is little substantial evidence or understanding of why. Therefore the majority of recent research can be characterised as having insufficient analysis, results or considerations of how and why multiple modes can assist in the resolution of errors and ambiguities. One obvious area of study is information redundancy between input modes. Such a study could give insights into the advantages of multiple input modes and provide more rigorous analyses.

\section{Redundancy}

A critical aspect of multiple input modalities is the concept of redundant information. A multimodal system can receive data from a single user for the same purpose from more than one source. If the input from one mode is successful in relaying the user's intentions then data from other sources is not required, and in such a case is redundant. However, if one information source fails, becomes corrupted or is of poor quality, then the presence of the previously redundant information may be pivotal for successful interpretation of the input message.

To illustrate the role of redundancy, consider the situation where a human being is communicating using multiple modes, for example, lecturing in a large hall. Assume they give a speech while pointing to a visual presentation and gesturing to highlight their talk. If the lights in the hall are switched off, the person can continue to speak and gesture, but only the speech will be 'received' by the audience. The lecture can still continue since the audience can hear. Alternatively, if the lecture hall became very noisy so that the speaker could not be heard, limited communication via gesture and the visual pictures on the screen could continue. Thus, the lecture could (in theory at least) be presented via only one of these modes, although we would all agree that the combination of both would be preferable for a successful lecture. If the 
two streams of similar information were available at all times throughout the lecture, one stream could be used to compensate for the other during times of distortion or of poor quality. This is the concept which can aid the analysis of the integration of multiple input modes and the benefits of multiple versus single modal input.

In the work of Vo and Waibel [26], the integration between speech and lipreading modes was examined. Tasks were first performed using speech input only followed by lip-reading input only. The same tasks were then performed using a combination of both the speech and lip-reading input. It was found that the recognition rates of the bimodal input were always better than or equal to either of the unimodal input rates. In particular, when the speech was corrupted with noise, the bimodal recognition rate was significantly higher than with speech alone. In later experiments, the integration of speech and lip-reading input gave a $9 \%$ error reduction over speech-only input for normal, 'clean' input, and a $29 \%$ error reduction over speech-only input for corrupted, 'noisy' input. These results were achieved even though the lip-reading input on its own gave only $12 \%$ recognition accuracy. Therefore, even the integration of poor quality streams of information provide benefits over the separate information streams.

Similarly in the work of Nakagawa et al [17], an experiment using speech and touch input revealed advantages in using these two modes against the use of speech input only. The bimodal input had higher recognition rates and lower error rates when compared to the unimodal speech input, illustrating again the co-operative and complementary nature of the two modes. The touch input served to enhance the available information when the speech input was poor or ambiguous. Thus, both ambiguities and errors were resolved through the use of two input channels. Clearly, redundancy has an important role to play in the integration of several input modalities.

It is interesting to note in these studies [17],[26] that the bimodal recognition figures are often higher than the unimodal rates but have never been higher than the sum of the unimodal rates. If this situation was to occur it would perhaps suggest that the combination of modes enables access to more information than is possible through any mode separately, and would provide undeniable evidence about the benefits of the integration of multiple input modalities.

\section{Initial Work}

A prototype multiple input system has been implemented with the complementary inputs of touch, two dimensional gesture and speech [21]. Currently, the speech and gesture recognition systems operate independently of one another. The current prototype is in the form of a shape-manipulation game (see Section 6). One of the main aims of the system is to prove that the concept of redundancy can be exploited in a simple, inexpensive multiple input system. We wish to demonstrate that a successful multimodal system can be built without access to extensive processing power, and most importantly, at time of poor input data quality. Because context is essential in input disambiguation, we also aim to achieve this success through having appropriate task knowledge available in the multimodal interpreter module (see Section 5). 
The multiple input system will be used to analyse the role of redundancy between input modes in producing tangible results about effects of integration of input modes and attempts to address questions such as

- Is there a level of redundancy at which optimal resolution of ambiguities is achieved?

- Are there modal performance levels at which redundancy has no/some/best effect?

- Can knowledge about the redundancy itself assist in the disambiguation process?

To illustrate our approach we will concentrate on the simple co-ordinate based two dimensional gesture recognition system which has been implemented using a pressure-sensitive touchscreen for touch and gesture input. The touch and gesture data from simple gesture experiments has been analysed using rule induction to give classification rules. Speech input is via a commercially available discrete voice recognition system. We use truth maintenance and blackboard system architectures to integrate the data from all inputs, production and heuristic rules, and task knowledge to form decisions and feedback from the system [14].

\subsection{Touch and Gesture Recognition System}

A two dimensional touch and gesture recognition system has been implemented. Gestures are made either by drawing with the mouse, fingertip, touch-pen or similar suitable light pressure contact on the touchscreen (Figure 1). The prolonged touch and drawing of the gesture on screen is regarded as a two dimensional gesture, which could be described as 'ergotic' gestures [24], as they represent actions in context of the system. Thus, the system accepts the input of two modes: touch and gesture.

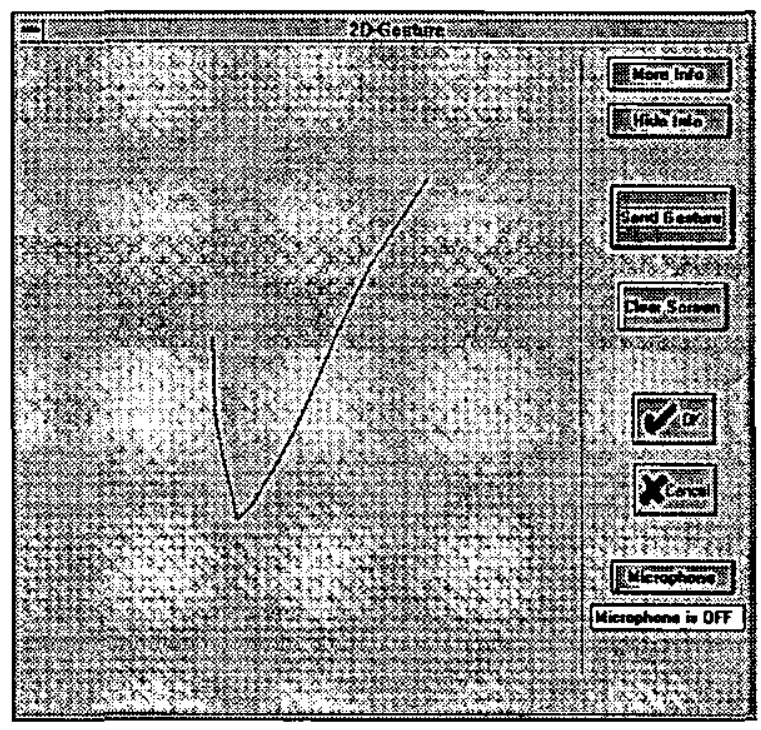

Fig. 1. Picture of touch and gesture input interface 
The co-ordinates are received by the system and lines drawn between consecutive points to form a flowing gesture on screen. The co-ordinates are recorded and used later for analysis in the gesture recognition system.

The gesture input is analysed by testing for a selection of geometrical patterns in order to decide the most likely shape that matches the gesture. Possible shapes that are looked for include: circle, tick', cross, or lines (vertical, horizontal, positive and negative gradients). Specifically, the pattern and percentages of gradients are analysed to gain an understanding of the gesture line content, gradient changes through the gesture, intersections between multiple gestures and gestures which selfintersect. Other attributes such as area, duration of gesture, time-stamps and measurements of distance between start and end points are also calculated.

This simple approach of co-ordinate tracking and analysis was chosen because processing of such input is simple and produces plentiful information attributes (currently fifteen attributes) for use in the analysis of the integrated input later in the research. The gesture recognition could have been performed in greater detail, using mathematical functions, or considering angles between line segments etc. [25]. However, the form of two dimensional gesture recognition described here is ideal for our experimentation needs later.

\subsection{Initial Results}

It has been observed that the quality of gesture input is affected by the medium used. For example, it is possible to make gestures with the mouse pointer as well as on the touchscreen. Mouse-created gestures are more precise in comparison to the gestures made using the touchscreen's 'fuzzy' input. Note the difference in Figures $2 \& 3$ :

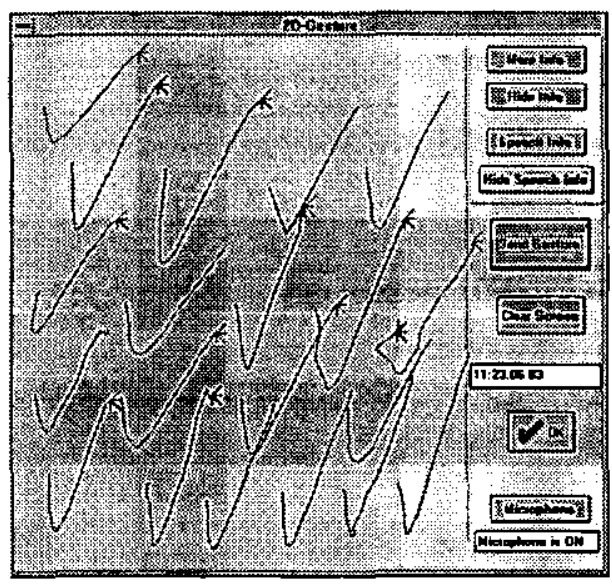

Fig. 2. Ticks made using touchscreen

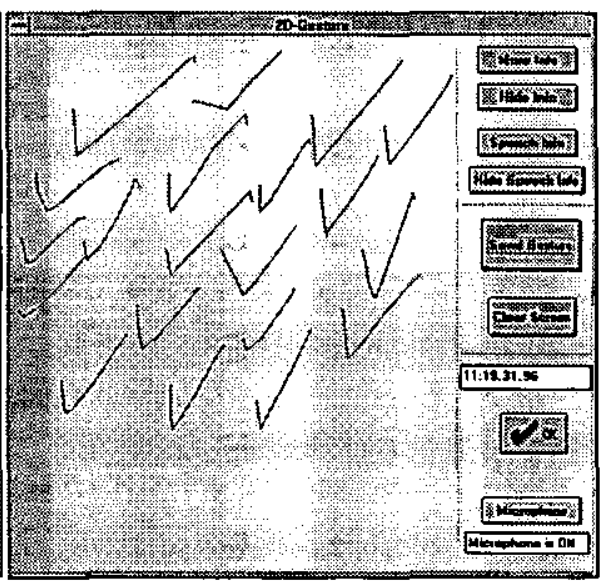

Fig. 3. Tick made using mouse

${ }^{1}$ It should be noted that a tick may also be known as a check mark, thus: 
Figure 2 shows gestures made with the touchscreen, with detected selfintersections plotted for information and program debugging purposes. The selfintersect test detects whether a single gesture has overlapped itself at any point. Figure 3 shows gestures made with the mouse, with the self-intersection plot also in operation (although, as can be seen, no self-intersects were detected in these mousecreated gestures). Results in Table 1 also show the differences between the two associated streams of data for the twenty ticks shown on each screen.

Table 1 shows that the various gesture recognition tests have varying success dependent upon the medium used. For instance, ticks can be gestured which we recognise as a tick visually regardless of the medium used but in which the gesture recogniser finds significantly differing characteristics. The medium used (touchscreen or mouse) is clearly responsible for many of these recognition variances.

It is interesting to note that no self-intersections are found in the gestures made by mouse whereas a high percentage of self-intersections are found in the gestures made by finger. This difference is explained by the pressure exerted on the touchscreen during gesturing with the finger. The pressure is detected by the touchscreen which attempts to plot the co-ordinates at the point where pressure has been detected. This can result in many points being detected by the system in a relatively small area of the screen (5-10 pixels wide). These points, when linked into a flowing gesture, are interpreted by the system as forming intersecting lines, therefore returning a positive result when the self-intersect test is performed. Obviously this pressure element is not present when the mouse is used.

\begin{tabular}{|l|l|l|l|l|l|}
\hline Gesture & $\begin{array}{l}\text { Positive } \\
>= \\
\text { Negative }\end{array}$ & $\begin{array}{l}{ }^{2} \text { Vertical } \\
>=\text { Horizontal }\end{array}$ & $\begin{array}{l}{ }^{3} \text { Gradient } \\
\text { change } \\
\text { found? }\end{array}$ & $\begin{array}{l}{ }^{4} \text { Line slope } \\
\text { detected? }\end{array}$ & $\begin{array}{l}{ }^{5} \text { Self- } \\
\text { intersect } \\
\text { found? }\end{array}$ \\
\hline $\begin{array}{l}\text { Ticks by hand, } \\
\text { (Figure 2) }\end{array}$ & $100 \%$ & $100 \%$ & $25 \%$ & $50 \%$ & $55 \%$ \\
\hline $\begin{array}{l}\text { Ticks by mouse, } \\
\text { (Figure 3) }\end{array}$ & $85 \%$ & $60 \%$ & $40 \%$ & $20 \%$ & $0 \%$ \\
\hline
\end{tabular}

Table 1. Percentage of gestures matching criteria

KEY:

1: \% of gestures with greater positive gradient content than negative gradient

2: \% of gestures with greater vertical gradient content than horizontal gradient

3: \% of gestures where negative to positive gradient change found

4: \% of gestures where significant sloped line content found

5: \% of gestures where a self-intersection has been found and plotted on-screen

The media used (touchscreen and mouse) have different properties (for example, method of input, rate and accuracy of data receipt) and it is this which perturbs the simple gesture recognition. The chosen solution for the two observed modally overlapping media (touchscreen and mouse) is to add further heuristic tests which desensitise the gesture recognition routine. This can be achieved because the two 
media have significant, but not extremely different performance parameters. Perhaps if performance parameters had differed significantly, two distinct recognition routines would have been necessary. This is perhaps a sub-set of a much larger issue of examining the implications of media with overlapping modalities.

\section{Touch and Gesture Experimentation}

To analyse the gesture recognition system for robustness and also gain understanding of possible patterns for use in multimodal integration, it was decided to use rule induction to generate classification models for the gestures. We also believe that by using rule induction we can capture an element of the variability of input and enhance our knowledge of possible error in the tasks.

\subsection{Experiments}

Two sets of touch and gesture only experiments (no speech) were therefore conducted to gain touch and gesture data using the 2D gesture recognition system described, in a simple graphical application. Ten users were involved per experiment. In both of the experiments users were asked to follow the on-screen requests (Figure 4) which involved the manipulation, by gesture only, of coloured shapes around a numbered grid (Figure 5).

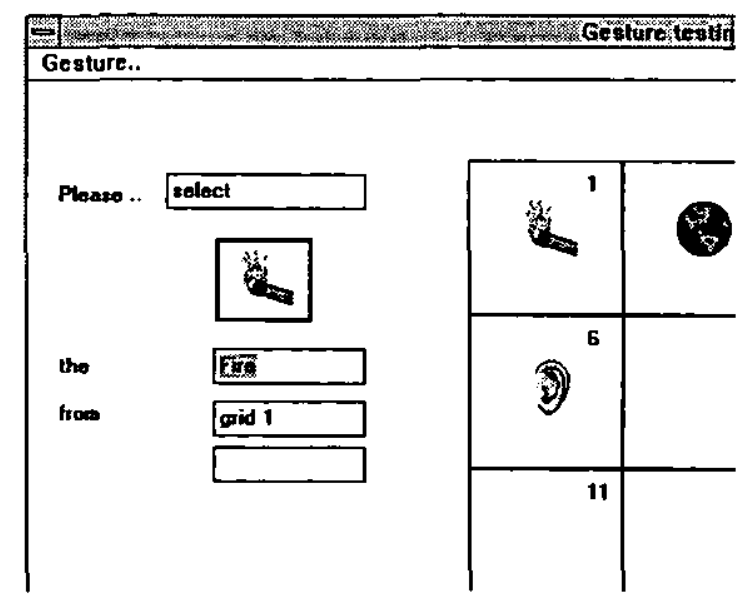

Fig. 4.: Partial screen detail of application, showing 'request area' and clipboard window (displaying matchstick icon)

Before the experiment, users were given instructions on: the number of gestures they would be prompted by the system to perform (128 gestures in each experiment); they were under no time restrictions; to use the touch-pen supplied (thus, achieving a compromise on the method of input, as the touch-pen, a simple inkless pen with a nib, 
gives better accuracy on the touchscreen than a finger); the mapping of pre-defined gestures to gesture commands. Thus, possible commands were cut, copy, paste and move. The appropriate gestures to perform to execute these commands were CROSS for cut (consisting of two separate line gestures which intersect one another), CIRCLE for copy; TICK for paste (where the currently selected object to paste is displayed on a clipboard window, see Figure 4); LINE for move (where the direction of the line necessary is obvious from the request). Thus the gestures intrinsically contain command information. Also, the gestures were in no particular order (generated by pseudo-random numbers). With 10 users per experiment, and 128 gestures per user, the total number of gestures for each experiment was 1280 .

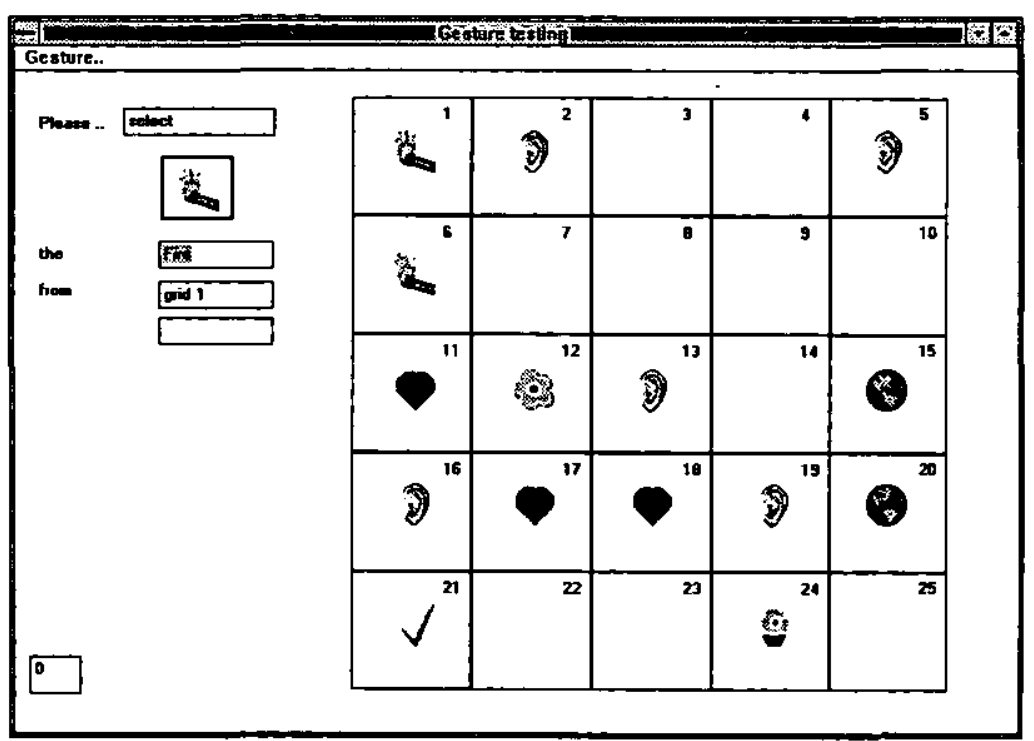

Fig. 5. Picture of gesture experimentation application screen

There was no error correction present in the first experiment. Errors arose when users did not follow the on-screen requests. For instance, the current request may have been for a copy/select gesture, and a user perhaps misread, or thinking about the previous request, performed a different gesture, such as a tick for paste instead. The second experiment therefore had error correction, this being the only difference between the experiments. Therefore, error-bound data was removed from the first dataset, giving a total of 923 cases. The second dataset had a complete set of 1280 cases (gestures).

\subsection{Use of Rule Induction}

The two datasets were analysed separately using rule induction to provide production rules. This gave us further insight into the robustness and consistency of the gesture 
This overall architecture (Figure 6) has been adopted for several reasons. Firstly, the data produced by the recognition systems can be grouped to suggest multiple recognition possibilities which are mutually inconsistent. Therefore, a resolution of these possibilities would be required. An assumption-based truth maintenance system (ATMS) can sustain several mutually inconsistent views [23], and is thus ideally suited to the needs of this research.

The ATMS to be utilised in this research is also combined with blackboard architectures and rating systems [14]. The blackboard architecture aids the efficiency of solving a problem while the rating system produces confidence values (weights) for the assumptions and also contributes to the system efficiency (response time etc.). These confidence values will perhaps prove valuable in the multimodal experimentation phase, as they may help to represent real-valued information about levels of redundancy. Finally, the use of task knowledge and conceptual dependency allows a flexibility in interpretation attempts by the multimodal interpreter for improved interaction, allowing for various speech, touch and gesture combinations (speech before or after gesture).

\section{Multiple Input Application}

The prototype multiple input system employs the speech and touch \& gesture recognition systems. The interface requires a level of complexity which will create possible ambiguities, requiring use of complementary and enhancing modal data for resolution of meaning. This approach is necessary for the multimodal experimentation phase which is to follow.

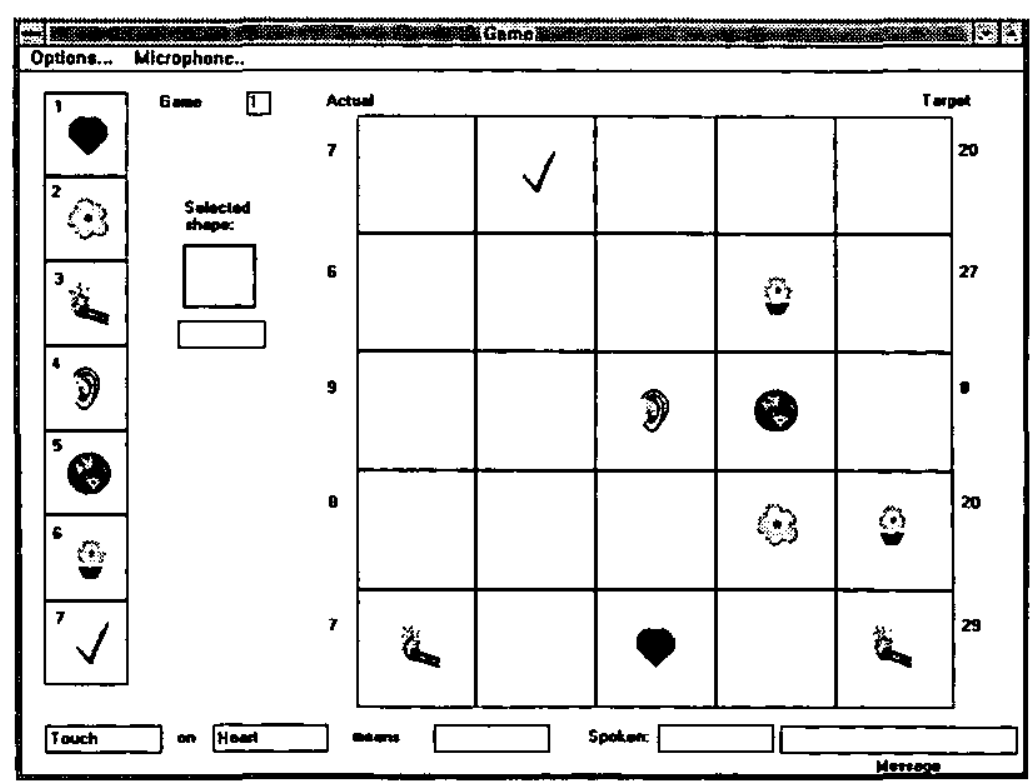

Fig. 7. Picture of prototype multimodal game interface 
For instance, ambiguities may arise when a spoken utterance rhymes with other recognition possibilities in the vocabulary. Knowledge of the associated touch and/or gesture is then required to resolve the interpretation e.g. 'move the STICK to grid SIX' and 'move the TICK to grid SIX' can confound the speech recognition since 'STICK' and 'TICK' may be confused with one another. The touch or gesture, in such a case, should support or contradict speech possibilities, by resolving the inilial target of the action, TICK or STICK. Therefore, it is vital to include the possibility of such ambiguities in the choice of objects in the application, vocabulary for the speech system and possible combinations of speech, touch and gesture.

The current prototype is in the form of a game (Figure 7), similar to the gestureonly system described in Section 4.1). In this multimodal prototype the aim is to manipulate coloured shapes (which have been assigned varying arithmetic values) around a grid, within certain constraints, using a combination of speech, touch and gesture. These constraints take various forms: a time limit; each row or column requiring the value of shapes within to reach certain arithmetic totals; only one of each shape in a particular row or column.

The interface comprises a grid and a toolbar where shapes may be selected. Actions can be performed in the system using the speech, touch and gesture modes separately as appropriate or in combination.. Tasks which can be performed include cut/delete, select/copy, paste and move (similar to tasks in touch and gesture only experimentation, Section 4.1). Basic feedback about the word spoken, possible action, shape chosen and the gesture made are displayed on screen. Currently, the speech and gesture recognition systems operate separately.

Speech recognition for the prototype system is performed by a commercially available discrete voice recognition system for Microsoft Windows. As with the gesture input, speech input has been analysed to obtain particular attributes: word recognised; percentage confidence score for this word; other recognition possibilities for the utterance; values scoring these possibilities and rejection score setting. Further work on this is to be reported separately. An assumption-based truth maintenance system combined with blackboard architectures has been developed, which will assist in integration of all inputs in the multimodal interpreter [14] (Section 5).

Currently tick, circle, line and cross gestures can be made on the toolbar or grid to manipulate items. Speech input is constrained to one-word utterances only. These utterances match the appropriate commands such as 'select', 'move', 'delete' , as well as vocabulary matching the shapes on screen: 'heart', 'circles' etc. Future work will include the interpretation of complex phrases of more than one word (within the constraints of the discrete speech recognition software) such as 'move-that-there' and similar placement oriented vocabulary.

\section{Future Work}

Future work includes analysis of speech data, the main multimodal experimentation, completion of the multiple input application and completion of the multimodal interpreter for modal integration.

Further analysis of the speech data will involve investigation of more information attributes which are critical to our approach. Further development of the 
prototype interface will involve creating a more complex environment in order to create scope for user errors, ambiguities and overlaps of meaning and intention. In the multimodal experimentation, distortions in the input channels could be made, thus changing the levels of redundant information and producing further artificial ambiguities. This will produce an ideal environment in which the effects of redundant and enhancing information in the integration of modes can be observed.

\section{Conclusions}

An overview and discussion of the need for the development of input methods in human-computer interaction has been presented. The benefits of multiple input modes over a single mode have been introduced. These benefits arise by mimicking the usual human complementary modes of speech and gesture which exploit redundant information. This concept of redundancy gives scope for error and ambiguity resolution.

Preliminary work on the touch and gesture recognition system has shown that some media may have overlapping modalities which affect the success of gesture recognition. It has been suggested that this could apply to other media and modes. A solution for improving gesture recognition in the presence of modally overlapping media is presented, but it is recognised that there may be many possible solutions. Further work using rule induction on gesture data has been discussed, with the production rules gained giving classification rates of around $80 \%$.

A proposal for a multimodal interpreter for the integration of speech, touch and two dimensional gesture using assumption-based truth maintenance and blackboard architectures has been presented. This approach will enable possibly inconsistent and redundant user input to be considered for recognition, with the rating system providing confidence scoring for individual parameters as well as providing system efficiency and realistic response times. The proposed use of a rule-based framework and the exploitation of task knowledge within this architecture has also been presented.

\section{References}

[1] Ando, H.; Kikuchi, H.; Hataoka, N.; Agent-typed Multimodal Interface Using Speech, Pointing Gestures and CG. Symbiosis of Human and Artifact; Anzai, Ogawa; Mori, eds.; pp29-34, 1995.

[2] Ando, H.; Kitahara, Y.; Hataoka, N.; Evaluation of Multimodal Interface using Spoken Language and Pointing Gesture on Interior Design System. International Conference on Spoken Language Processing, pp567-570, 1994. 
[3] Bellalem, N.; Romary, L.; Structural Analysis of Co-verbal Deictic Gesture in Multimodal Dialogue Systems. Proceedings of Gesture Workshop 1996: Progress in Gestural Interaction, pp141-153, Springer Verlag, 1997.

[4] Bellik, Y; Modality Integration: Speech and Gesture. Survey of the State of the Art in Human Language Technology; Cole, R, ed.; Available from the Centre for Spoken Language Understanding, Oregon Graduate Institute, USA, 1997.

[5] Blake, A.; Isard, M.; Reynard, D.; Learning to track the visual motion of contours. Artificial Intelligence, No. 78, pp179-212, 1995.

[6] Bolt, R.A.; The Human Interface - where people and computers meet, Chapter 3, pp35-52, 1984.

[7] Bordegoni, M.; Parallel Use of Hand Gestures and Force-Input Device for Interacting with 3D and Virtual Reality Environments. International Journal of Human-Computer Interaction, Vol. 6, No. 4, pp391-413, 1994.

[8] Bordegoni, M.; Faconti, G.P.; Architectural Models of Gesture Systems. Proceedings of Gesture Workshop 1996, pp61-73, Springer Verlag, 1997.

[9] Bregler, C.; Manke, S.; Hild, H.; Waibel, A.; Bimodal Sensor Integration on the Example of "Speech-Reading". Proceedings of the International Conference on Neural Networks, 1993.

[10] Caloini, A.; Tosolini, P.; Time and gesture based interfaces a test case: Shinjuku Guide. UK Toolbook User Conference, 1994.

[11] Davis, J.; Shah, M.; Gesture Recognition; Technical Report available from Department of Computer Science, University of Central Florida, Orlando, FL 32816.

[12] Duchnowski, P.; Meier, U.; Waibel, A.; See me, Hear me: Integrating Automatic Speech Recognition and Lip-reading. ICSLP '94, International Conference on Spoken Language Processing, pp547-50, Vol. 2, 1994. 
[13] Fishman, E.S.; Padilla, E.; Speech Recognition Software Overview Summer/Fall 1997. Speech Recognition Update August 1997. Available from http://www.voicerecognition.com/article_8_97.html.

[14] Hinde, C.J.; Bray, A.D.; et al; A Truth Maintenance Approach to Process Planning. Artificial Intelligence in Manufacturing, Proceedings of the $4^{\text {t }}$ International Conference on the Applications of Artificial Intelligence in Engineering, pp171-188, 1989.

[15] Koons, D.B.; Sparrell, C.J.; Thorisson, K.R.; Integrating Simultaneous Input from Speech, Gaze, and Hand Gestures. Intelligent Multimedia Interfaces, pp257-276, AAAI Press, 1993.

[16] Markowitz, Judith; Talking to Machines. Byte, pp97-104, December 1995.

[17] Nakagawa, S.; Zhang, J.; Chengcharoen, W.; A multi-modal Interface with Speech and Touch Screen. Symbiosis of Human and Artifact, pp213-218, Anzai; Ogawa; Mori, eds.; 1995.

[18] Newby, G.B.; Gesture Recognition Using Statistical Similarity. Proceedings of the Virtual Reality and Persons with Disabilities Conference, 1993.

[19] Oviatt, S.; Multimodal Interactive Maps: Designing for human performance. Human-Computer Interaction, in press.

[20] Oviatt, S.; DeAngeli, A.; Kuhn, K.; Integration and Synchronization of Input Modes during Multimodal Human-Computer Interaction. CHI 97, Atlanta GA, USA. 22-27 March 1997, pp.415-422.

[21] Oviatt, S.; Olsen, E.; Integration Themes in Multimodal Human-Computer Interaction. Proceedings of International Conference on Spoken Language Processing, pp551-554, 1994.

[22] Oviatt, S.; VanGent, R.; Error resolution During Multimodal Human-Computer Interaction. Proceedings of International Conference on Spoken Language Processing, 1996.

[23] Rich, E; Knight, K; Artificial Intelligence, McGraw-Hill, 1991. 
[24] Robbe, S.; Carbonell, N.; Dauchy, P.; How Do Users Manipulate Graphical Icons? An Empirical Study. Progress In Gestural Interaction. Proceedings of Gesture Workshop 1996. Springer, 1997.

[25] Rubine, D.; Specifying Gestures by Example. Computer Graphics 25, 4, pp 329-337, ACM Press, 1991.

[26] Vo, M.T.; Waibel, A.; Multimodal Human-Computer Interaction. Draft available from Alex Waibel at School of Computer Science, Carnegie Mellon University, Pittsburgh, 


\title{
Integrating Speech and Two-dimensional Gesture Input - A Study of Redundancy between Modes
}

\author{
Karen McKenzie Mills \& James L. Alty \\ Dept. of Computer Studies, Loughborough University, UK. \\ \{K.C.M.Mills, J.L. Alty\}@lboro.ac.uk
}

\begin{abstract}
It is widely recognised that multiple modes of input give higher recognition rates than single modes. The use of multiple modes, such as speech and gesture, can allow for more natural human-computer interaction. Opportunities for error and ambiguity resolution also occur, as the same information may be available from more than one source, providing redundant or repeated information. This paper presents a study of the redundant integration of speech and twodimensional gesture input in a relatively simple, inexpensive multimodal input system. Empirical results about multimodal recognition rates at particular levels of redundancy are presented and implications for the integration of multiple modes are discussed.
\end{abstract}

Keywords: Speech, Gesture, Multimodal, Input, Integration.

\section{Introduction}

In human-human interaction multiple communication modes such as speech, gestures and body movements are frequently used. By mode, we mean a unique communication or interaction style (audio vs. video, written vs. spoken etc.). Generally, humans are successful at communicating their intentions, actions and feelings to one another, because they use a variety of modes in combination (sometimes redundantly). Current human-computer interaction uses a number of modes to effect communication (input and output). Whilst the modes used for output communication are a reasonable match to those used by humans, current input modes are more limited. The standard input methods used, such as text input via the keyboard and pointer/location information from a mouse, do not provide the optimum communication environment. Flexible input combinations more suited to human abilities (e.g. speech and gesture modes) are not yet a standard desktop combination for humancomputer interaction. In addition to providing a more natural interaction, the use of multiple modes also enhances the input recognition task $[3],[4],[5],[7],[11],[12]$.

In early studies of modal integration, multimodal recognition levels were higher than the single mode rates [1],[10],[16] even when one mode was performing poorly. This benefit was attributed to the presence of redundant information across the modes, which was called upon, at times of ambiguity, to resolve an outcome [14],[15].

The study presented here is an empirical investigation of the role of redundancy in multimodal recognition efficiency. The input modes used in this study are speech, and two-dimensional gesture. Speech recognition is performed by a commercial discrete software recognition package [17], while a two-dimensional gesture recognition system was implemented for this research [6] (which uses a truth maintained blackboard system [9]). A multimodal integrator which combines speech and gesture input has been developed, whilst a shape movement game has been implemented, under the MS Windows 3.1 environment, to provide an application framework.

\subsection{Redundancy}

In human-computer systems consisting of more than one mode of input, the possibility of repeated or redundant information is an important source for resolving input ambiguity. A multimodal input system can receive data from a single user for the same purpose, from more than one input source. If the input from one mode is successful in relaying the user's intentions, then data from other modes is not required, and in such cases is redundant. However, if one information source fails, becomes corrupted, or is of poor quality, then the presence of information from a previously redundant mode may be pivotal for successful interpretation of input.

There are several outcomes that might be expected from an implementation of a speech and gesture input system, investigating the role of redundancy across these modes. Firstly, that multimodal recognition success would be expected to be higher than either the speech or gesture recognition rates alone. Secondly, it 
might be expected that particular levels of redundancy would provide optimum multimodal recognition figures.

\section{Speech and Gesture Input Study}

In order to create an experimental environment, an application was developed, displaying a numbered grid on which objects are manipulated by a combination of speech and gesture (Figure 1).

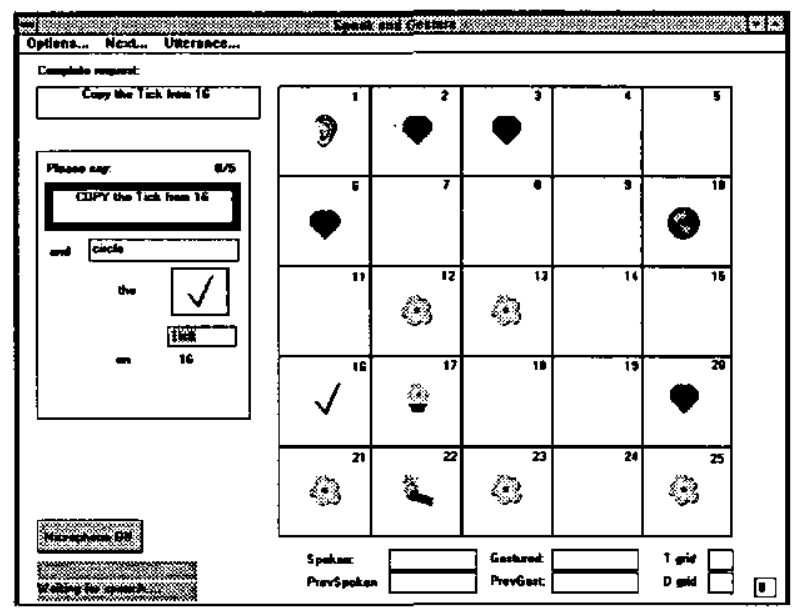

Figure 1. Experimentation interface

\subsection{Multimodal Tasks}

Users were asked to follow an on-screen guide which generated speech and gesture tasks (see Figure 2 below). The tasks required users to cut, copy, paste and move the objects around the grid. The tasks were presented on-screen in the form 'Cut the Heart from 6', 'Paste the Earth to 25', 'Move the Ear from 12 to 3', etc.

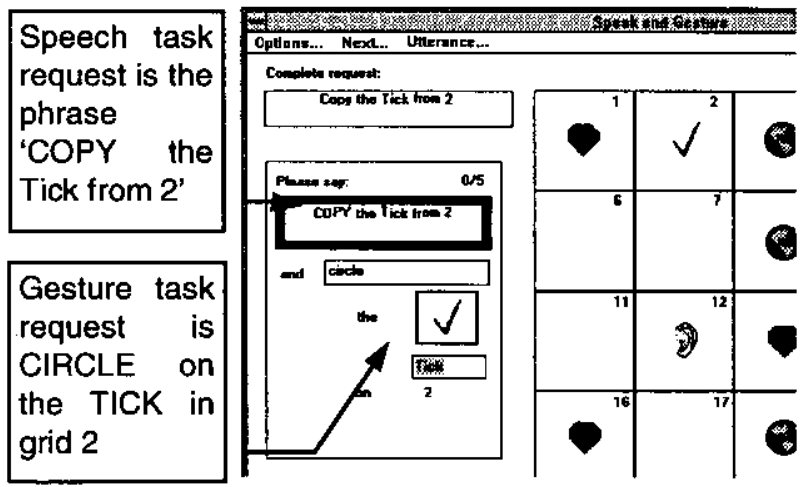

Figure 2. Request area of experimentation interface

Therefore, the multimodal task involved the sequential use of speech and gesture. Whether input is simultaneous or sequential, additional information is available because of the presence of more than one input mode. Sequential input was used in this system due to the processing limitations of the PC platform. The temporal occurrence of input (in the context of this system) does not affect the benefits of these additional input modes, as shown in Section 3.5 Analysis of Multimodal Scores).

After the user had performed speech and gesture as requested, the system interpretation of the users' input could then be compared to the requested input. The request is regarded as the correct answer against which success is measured. This format of automatic requests is similar to earlier gesture-only experiments conducted [9].

The system had to provide an environment for investigating redundancy, by allowing for the repetition of input information across modes. Therefore, an equivalent semantic form of input across the modes was required to ensure redundancy. In the system three basic information fields must be filled for a complete input interpretation: command slot, object slot and location slot(s). Gesture input contained information which was a combination of all of these slots, whilst the speech input was a combination of some or all of these slots. The use of such slots is discussed in the next section.

\subsection{Design To Investigate Redundancy}

In the system, users were asked to follow action requests which were a combination of command, object and location(s) information. Speech and gesture requests were presented on-screen simultaneously and users were asked to perform the speech input first, followed by gesture input when the utterance was complete. In some cases the speech request was equivalent to the complete action, whilst at other times the speech request may have been a single word or number.

Therefore it was theoretically possible, in some cases, for the speech input to complete the multimodal slots, without reference to the gesture input. In all cases, it was possible in theory for the gesture input to complete the multimodal slots, without reference to the speech input. All gesture cases had the capability to provide this, as there was no variance of information content in the gesture input (see Section 2.4). Based on these considerations, speech utterances were divided into three categories: single, partial and complete utterances, representing various types of slot repetition. Therefore, a variety of combinations of speech and gesture provided different levels of 
repeated input information, and hence varying levels of redundancy in the input was achieved.

Speech utterances consisted of single words or phrases of up to seven words (e.g. 'Move the Heart from 8 to $10^{\prime}$ ). Speech recognition was provided by a discrete voice recognition software package which gave speech data, used to fill the speech information slots of command, object and location(s). Similarly the gesture data was processed in real time using a truth maintenance and blackboard system as part of a two-dimensional gesture recognition system [6],[9]. This provided gesture recognition possibilities and weightings for those possibilities. This data then filled a set of gesture information slots, again, command, object and location(s). The approach is similar to the natural language processing technique of conceptual dependency. A conceptual dependency framework represents every action as being composed of component parts, which can fill a constant set of slots, regardless of the differences in the description of that action [2],[13]. Thus the multimodal slots of our system were the constant structure that was filled from both speech and gesture input.

\subsection{Integration Method}

The processed speech and gesture information was sent to the multimodal integrator for combination and validity checks. Three basic possibilities were supported in the multimodal integrator: where recognised speech and gesture information agreed; where conflicts occurred between the speech and gesture; and where only partial information was available across the modes.

The 'best' speech and gesture choices were determined by the weights which they were assigned in the recognition process. The weights provided a guide about how confident the system was about those recognition possibilities, and allowed comparison of input. If conflicts arose between the modes, the speech or gesture possibility with the highest confidence rating was chosen.

The generated multimodal interpretation containing information from the speech choice and the gesture choice, had overall multimodal information slots relating to command, object and location information. This possible multimodal combination was checked for validity, and if true, the process stopped. If the possibility was false, the next gesture choice and the current speech choice were sent through the combination process again. If none of the gesture choices made a valid combination with the given speech choice, the next speech choice was used. Thus, a simple linear search of the two-dimensional space was performed until a valid combination was found.

The validity check is the process whereby the validity of the value of each combination of information slots is checked against the current system knowledge. This consists of explicit and implicit system knowledge such as current object positions, the relevance of objects to commands (e.g. pastes are invalid if there is no object on the clipboard) and, for commands, whether the location information is valid (e.g. moves must have two location information slots to be valid).

Thus, the integration of speech and gesture in the multimodal input system was performed by combining a speech option (there are at most ten possibilities) with a gesture option (again, there are at most ten) until a valid combination was found which was consistent with the system knowledge.

\subsection{Varying levels of Redundancy}

One way to vary the redundancy or repetitiveness of information in the system was to vary the speech input to the system, whilst maintaining the level of information contained in the gesture. It was preferable to maintain the level of information in the gesture due to the inherent command-based nature of the twodimensional gesture itself [8]. Decomposition of a gesture into specific component parts (command only, destination only etc.) is more complex than the equivalent decomposition of a speech phrase, since a 'command-less' or 'object-less' gesture (i.e. only involving a selection of parameters) is difficult to simulate.

Speech input consisted of either a single word, or a phrase of up to seven words. The use of speech phrases in addition to single words gave greater scope for varying the meaningful information that the system could interpret.

Some examples of speech requests, containing various levels of information, are shown in Table 1.

Table 1: Examples of Speech Requests 


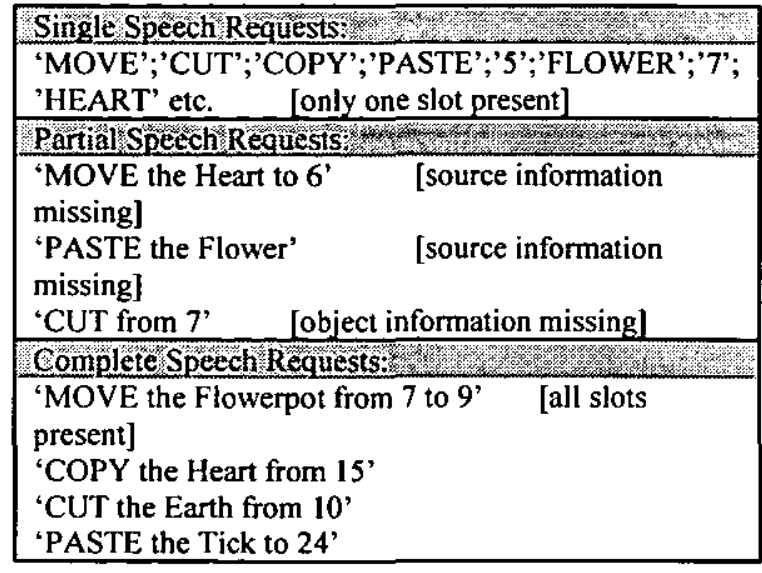

Across the whole experiment $1 / 3$ of speech requests were single, $1 / 3$ were partial and $1 / 3$ were complete.

A redundancy score was devised based on the number of slots repeated within a construct. A redundancy score of $100 \%$ represented a repetition of all slots in a construct, that is, speech requests contained the same information as the gesture requests. In this case, either mode could theoretically contain adequate information to perform the overall action. As the redundancy score decreases, the possibility of the speech mode theoretically containing enough information to perform the overall action also decreases. Thus a redundancy score represents only the slots already present in the gesture which have been repeated in the speech. The scale of redundancy scores is displayed in Table 2.

\section{Table 2: Possible redundancy scores}

\begin{tabular}{lllll}
\hline $\begin{array}{l}\text { Speech } \\
\text { Request } \\
\text { Type }\end{array}$ & $\begin{array}{l}\text { Cut/Copy/ } \\
\text { Paste }\end{array}$ & $\begin{array}{l}\text { \# of slots } \\
\text { repeated }\end{array}$ & Move & $\begin{array}{l}\text { \# of slots } \\
\text { repeated }\end{array}$ \\
\hline Single & $33 \%$ & $1 / 3$ & $25 \%$ & $1 / 4$ \\
Partial & $66 \%$ & $2 / 3$ & $75 \%$ & $3 / 4$ \\
Complete & $100 \%$ & $3 / 3$ & $100 \%$ & $4 / 4$ \\
\hline
\end{tabular}

\subsection{Experimental Format}

Thirty users were involved in the main experiment. Each user was asked to follow the on-screen guide which generated (in a pseudo-random combination and order) speech and associated gesture instructions. Before beginning the shape game, users were asked to train the speech software on a selection of words associated with the application. This gave users the opportunity to familiarise themselves with the use of the microphone and speech recognition and also provided vocabulary files that the application used in recognising speech during the main experiment.

In total each user was asked to perform 128 combinations of speech and gesture, split over two sessions for manageability. Speech input was through the microphone with discrete voice recognition software, whilst gesture input was made with a touchpen on a pressure-sensitive touchscreen. No time restrictions were imposed upon users although other restrictions were in force, as described below.

Each combination of speech and gesture required that speech be performed before the gesture. Further, as the voice recognition software was discrete, users were asked to pause slightly between each word in a speech utterance. A word count in the guide window provided help with orientation of which word had been uttered and received by the system.

Users were given instructions before the experiment on these restrictions. They also received instructions on the pre-defined gestures and the associated commands of a cross for cut, circle for copy, tick for paste, and line for move (cut, copy and paste defined as in standard text editing meaning).

\section{Results}

\subsection{Subjects}

All thirty subjects were from a variety of different educational backgrounds, none of whom used speech or gesture input technology to any significant degree. Subjects had a wide variety of accents, with 23 having British accents, while 7 subjects had non-British accents. Since the speech recognition software was optimised for British accents, it may have been reasonable to expect lower recognition figures for those subjects with non-British accents. As the results show in the next section however, there was not a significant difference in the speech recognition figures between the British versus non-British speech figures.

\subsection{Errors made by subjects during interaction}

During interaction some users did become confused, performing actions contrary to those requested. For example, in a case where the overall action had the format of -Move the Heart from 6 to 9-, some users performed the requested gesture backwards and so gestured from grid 9 to 6 , when the correct gesture would have been from grid 6 to grid 9 . However, as the occurrence of errors was relatively rare, such errors were left in the datasets. They could be regarded as a challenge to the system to analyse how it coped with the possible contradictions that these errors could generate.

\subsection{Analysis}


In the following sections the data from the main experiment is analysed for significant differences in the means of the three average recognition scores obtained: speech, gesture and multimodal. Descriptive statistics are shown where appropriate, statistical tests used are described and significance levels are also shown. Where appropriate, further analysis is presented.

\subsection{Initial Expectations}

From the experiment, three main results were expected. It was expected to find that the multimodal recognition rates would be higher than speech or gesture recognition rates. It was also expected to find that there would be a linear correlation between the coarse redundancy levels and the multimodal recognition rates. Finally, it was expected to find that within the coarse redundancy levels, the differing slot repetition types would give distinct multimodal results for each category. From these results it would be expected to formulate where the multimodal gain occurred and hypothesise on guidelines for multimodal integration.

\subsection{Analysis of Multimodal Scores}

The average speech, gesture and multimodal scores obtained from 30 subjects were analysed. A boxplot showing the distribution of the recognition data is shown in Figure 3.

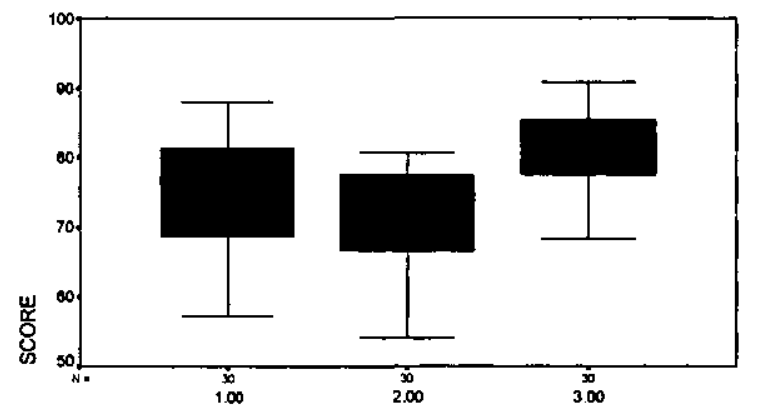

MODE

Figure 3: Boxplot of average speech (mode $=1)$, gesture $($ mode $=2$ ) and multimodal (mode=3) scores from $\mathbf{3 0}$ subjects

The boxplot shows that there are no outliers or extreme values in the three sets. Both speech and gesture rates (mode $=1$ and 2 respectively) have slightly skewed distributions as seen by the position of their medians and the placement of the first and third quartiles, that is, the boundaries of the surrounding boxes. The multimodal rates $(\operatorname{mode}=3$ ) have less variability than either speech or gesture (indicated by the length of the box). Therefore, as the data has no extreme values or oddities we can proceed with further analysis.

The results for 23 out of 30 subjects gave higher average multimodal recognition scores than either speech or gesture recognition scores (see Section 3.6 for details of the seven cases with lower multimodal scores). The boxplot in Figure 3 also seems to suggest that, overall, multimodal recognition rates are higher. To confirm this, a comparison of the means (for all 30 samples) was necessary to test the hypothesis that there was a significant difference in the means of the three variables.

Table 3: Descriptive Statistics for Average Speech, Gesture and Multimodal Recognition Scores

\begin{tabular}{llllll}
\hline & $\mathrm{N}$ & Mean & Std Dev & Min. & Max. \\
\hline Speech & 30 & 75.62 & 8.70 & 57.29 & 88.09 \\
Gest & 30 & 71.49 & 7.12 & 54.10 & 80.60 \\
Mult & 30 & 81.67 & 6.08 & 68.29 & 90.89 \\
\hline
\end{tabular}

Therefore an ANOVA (analysis of variance) could provide the method for hypothesis testing. First of all, the descriptive statistics shown in Table 3 require analysis.

The descriptive statistics show that the standard deviations of the three variables (speech, gest and mult) are within the range for application of a standard one-way ANOVA, where the largest standard deviation is no more than twice as large as the smallest standard deviation (and also assuming the data is from a normally distributed population).

The results of the ANOVA give an $F$ statistic of 14.46 at a significance level of $p<0.0001$ $(F(2,87)=14.46, p<0.0001)$. If there was no difference between the groups the $F$ statistic should approximate close to the value 1 . Therefore, it can be concluded that there is a significant difference between the three groups of speech, gesture and multimodal scores. The follow up Bonferroni test gives an indication of which groups are significantly different from one another at the $\mathrm{p}<0.05$ level, shown in Table 4 .

\section{Table 4: Bonferroni Test for 30 subject main} experiment

\begin{tabular}{lll}
\hline & & GRP 1 GRP 2 GRP 3 \\
\hline Mean & MODE & \\
75.6145 & Grp 1 (Speech) & \\
71.4890 & Grp 2 (Gesture) & \\
81.6694 & Grp 3 (Mult) & $*$ \\
\hline
\end{tabular}

The result of the Bonferroni test indicates that the mean of speech scores (Mode=Grp 1) is significantly different from multimodal scores (Grp 3). Also, the gesture scores (Grp 2) are significantly different from 
multimodal (Grp 3) scores. The speech and gesture scores (Grps 1 and 2) are not significantly different from one another. This lack of difference between speech and gesture is not significant however. Crucially, it is the multimodal scores which are significantly different, and higher than, the speech and gesture scores.

Thus, for the 30 subject main experiment it can be concluded that the multimodal recognition scores are significantly higher than either speech or gesture recognition scores at a significance level of $p<0.05$.

\subsection{Special cases of multimodal scores}

As shown in the previous section, an analysis of variance has shown that the multimodal scores are significantly higher than speech or gesture scores.

Despite this statistical analysis however, there were 7 cases of the 30 subjects where the multimodal recognition rates had not improved on the speech or gesture rates. These cases are shown in Table 5.

Table 5: Seven cases from 30 subjects where Multimodal Rate not the highest

\begin{tabular}{llllllll}
\hline User \# & 2 & 5 & 7 & 17 & 18 & 22 & 28 \\
\hline Speech \% & 76.5 & 78.8 & 84.0 & 60.2 & 85.8 & 81.1 & 84.1 \\
Gesture \% & 68.1 & 54.1 & 66.5 & 79.4 & 69.5 & 57.7 & 64.8 \\
Multimodal\% & 74.4 & 72.5 & 79.4 & 78.9 & 81.6 & 74.9 & 78.1 \\
Accent Code & $o$ & br & 0 & br & br & br & br \\
\hline
\end{tabular}

Further analysis was required to attempt to identify reasons why these cases had given a lower multimodal rate. In six of the seven cases, the multimodal rate was higher than the gesture rate but lower than the speech rate. One case gave the multimodal rate higher than speech but lower than gesture. Did any particular circumstances occur in these cases that reduced the multimodal rate? What did the data reveal? Descriptive statistics for these seven cases indicated that a standard ANOVA was not possible as the range of standard deviations violated the standard assumptions (i.e. the variances were not approximately equal).

Table 6: Descriptive Statistics for 7 cases

\begin{tabular}{lcllll}
\hline & $N$ & Mean & Std Dev & Min. & Max. \\
\hline Speech & 7 & 78.6429 & 8.7607 & 60.2 & 85.8 \\
Gesture & 7 & 65.7286 & 8.2451 & 54.1 & 79.4 \\
Multimodal & 7 & 77.1143 & 3.2421 & 72.5 & 81.6 \\
\hline
\end{tabular}

Therefore a Kruskal-Wallis One-Way ANOVA was performed on the cases giving $\chi 2=7.9885, \mathrm{p}<0.05$, indicating that there was some differences between the speech, gesture and multimodal rates. The mean ranks for speech, gesture and multimodal rates were 14.86 , 5.79 and 12.36 respectively.

An analysis of the data in the seven cases found that the speech and multimodal scores were not significantly different at the $p<0.05$ level. Gesture scores were shown to be significantly different from both speech and multimodal scores, seen in the differences in values of the mean rank. The mean gesture score from these 6 cases is 63.5 , lower than the 30 subject average of 71.5. Therefore, a common factor in these cases is the relatively poor performance of the gesture recognition which the speech recognition has not compensated for, perhaps giving rise to the lower multimodal scores. Section 3.8 presents further explanations for these cases.

\subsection{Multimodal Scores and Redundancy}

To explore the relationship between the differing redundancy levels and the final multimodal scores, it was desirable to demonstrate some effect between them. Therefore analysing the sample means of multimodal scores in groups according to their redundancy levels, would provide a general overview of possible relationships, patterns and effects.

Redundancy could be quantified in several ways. As shown previously in Table 2, redundancy could be expressed as one of five possible values: $25 \%, 33 \%$, $66 \%, 75 \%$ or $100 \%$.

Several sub-categories within these categories provided further groupings. For instance, three types of speech repetitions produced $25 \%$ redundancy: repetition of commands, objects and destination slots within move actions. The relationships between the sub-categories are shown in Table $7(\mathrm{C}=$ command, $\mathrm{O}=$ object, $\mathrm{T}=$ target, $\mathrm{D}=$ destination).

\section{Table 7: Redundancy scores and repeated speech slots

\begin{tabular}{ll}
\hline Repeated Slots & Redundancy level \\
\hline C25, O25, D25 & $25 \%$ \\
C33, O33, T33 & $33 \%$ \\
CO, CT & $66 \%$ \\
COD, COT75 & $75 \%$ \\
COT100, COTD & $100 \%$ \\
\hline
\end{tabular}

The sub-categories were labelled according to the repeated slots and the overall redundancy level they belonged to. For instance, the case $\mathrm{CO}$ represents the speech requests consisting of command and object combinations. CT represent command and target combinations. Where possible ambiguity exists about the overall redundancy level, the level is appended to the combination e.g. COT75 represents command, object and target combinations for move actions ( 3 out 
of 4 , or $75 \%$ of slots have been repeated). COT100 represents the command, object and target combination for cut, copy and paste actions.

Using these sub-categories, a greater level of detail could be applied to the analysis of multimodal scores. Firstly, a test for correlation between five redundancy levels and multimodal scores was performed, giving $r=0.2109$ at $p<0.0001(N=360)$. As the sample is large, this is not a significant figure and scatterplots of multimodal scores and redundancy values indicate that the relationship is not linear. Similarly for twelve subcategories, the correlation coefficient of $r=0.1286$ at $\mathrm{p}<0.02(\mathrm{~N}=360)$ indicates no linear relationship.

Further analysis to determine other relationships between redundancy and multimodal scores was therefore required. The five main redundancy levels gave multimodal results with smaller variances that either speech or gesture scores, highlighting the greater stability of the multimodal scores when compared to speech and gesture. The speech results had the highest variance levels, with the highest number of extreme values and outliers (17 for speech versus 4 for gesture and 9 for multimodal). Grouping multimodal results by the twelve redundancy categories also produced a wide distribution of scores.

Therefore, were the multimodal scores higher than either speech or gesture scores in each of the redundancy levels? Did a particular level of redundancy give optimum multimodal scores? Comparison of speech, gesture and multimodal scores was necessary per slot to determine if multimodal scores were highest. An analysis of average multimodal scores from each redundancy level was also necessary to determine if an optimum rate existed.

Therefore, to test the hypothesis that various levels of redundancy had an effect on the multimodal rates produced, a series of ANOVAs were performed. The results are shown in Table 8. Non-parametric one-way ANOVAs (Kruskal-Wallis one-way ANOVA) were used since the samples had uniform or non-normal distributions. The ANOVA results are shown as $\chi^{2}$ figures in the tables. The table shows overall results per redundancy level with further details for each sublevel.

Table 8: ANOVA results for multimodal scores with 5 main redundancy levels, 12 sub-levels

\begin{tabular}{|c|c|c|c|}
\hline $\begin{array}{l}\text { Redundan } \\
\text { cy level }\end{array}$ & $\begin{array}{l}\text { ANOVA } \\
\text { results }\end{array}$ & $\begin{array}{l}\text { Significance } \\
\text { level }\end{array}$ & $\begin{array}{l}\text { Significant } \\
\text { differences }\end{array}$ \\
\hline $25 \%$ & $\chi^{2}=1.3866$ & $p=0.4944$ & no sig. diff.at $p=.05$ \\
\hline $\mathrm{C} 25$ & $\chi 2=8.34$ & $\mathrm{p}=0.0154$ & no sig. diff. at $\mathrm{p}=.05$ \\
\hline $\mathrm{O} 25$ & $\chi^{2}=6.32$ & $p=0.0426$ & no sig. diff. at $\mathrm{p}=.05$ \\
\hline D25 & $\chi^{2}=59.75$ & $p<0.0001$ & $\begin{array}{l}\text { no sig. diff. at } \mathrm{p}=.05 \\
\text { between gest \& mult }\end{array}$ \\
\hline $33 \%$ & $x^{2}=24.1005$ & $p<0.0001$ & Multimodal highest \\
\hline $\mathrm{C} 33$ & $x^{2}=61.03$ & $p<0.0001$ & Speech highest \\
\hline $\mathrm{O} 33$ & $\chi^{2}=29.64$ & $\mathrm{p}<0.0001$ & Speech highest \\
\hline T33 & $x_{2}=60.41$ & $p<0.0001$ & Multimodal highest \\
\hline $66 \%$ & $\chi^{2}=111.81$ & $p<0.0001$ & $\begin{array}{l}\text { Speech and Mult not } \\
\text { sig. diff }\end{array}$ \\
\hline $\mathrm{CO}$ & $\chi 2=54.42$ & $\mathrm{p}<0.0001$ & Multimodal highest \\
\hline CT & $\chi 2=62.20$ & $p<0.0001$ & Speech highest \\
\hline $75 \%$ & $x^{2}=8.34$ & $\mathrm{p}=0.0154$ & no sig. diff. at $p=.05$ \\
\hline COT75 & $\chi^{2}=5.63$ & $\mathrm{p}=0.0601$ & no sig. diff. at $p=.05$ \\
\hline COD & $x 2=5.02$ & $\mathrm{p}=0.0815$ & no sig. diff at $\mathrm{p}=.05$ \\
\hline $100 \%$ & $x^{2}=51.44$ & $p<0.0001$ & Multimodal highest \\
\hline COT100 & $\chi 2=59.27$ & $\mathrm{p}<0.0001$ & Multimodal highest \\
\hline COTD & $x 2=2.1515$ & $\mathrm{p}=0.3410$ & no sig. diff \\
\hline
\end{tabular}

The results show that distinct redundancy levels did produce different multimodal results. The redundancy levels of $33 \%, 66 \%$ and $100 \%$ gave multimodal scores higher than either speech or gesture alone. In the other cases of $25 \%$ and $75 \%$, no significant statistical difference between speech, gesture and multimodal scores was identified.

Within the five main redundancy levels, the sublevels indicated some cases where speech recognition scores gave higher rates than multimodal rates. A possible explanation for the poor results from the $25 \%$ and $75 \%$ cases may be attributed to the fact that they represent the move actions. The move actions had a higher number of slots to fill than other cases (4 instead of 3) which may have produced greater scope for ambiguity and disagreements of the information across modes.

To discover whether an optimum multimodal rate was produced from any particular redundancy level, a non-parametric ANOVA was performed on the average multimodal scores. The results are shown in Table 9.

The results of the ANOVAs in Table 9 indicate that there were significant differences between the groups. The mean ranks show that a redundancy level of $66 \%$ gives the significantly highest multimodal score, closely followed by the $100 \%$ redundancy level. This is reflected in the detailed redundancy level where the $\mathrm{CO}$ redundancy slot (command and object slots were repeated in cut, copy and paste actions) gave the highest multimodal scores, again closely followed by the COT100 slot (command, object plus target slots repeated in cut, copy and paste actions). 
Table 9: Kruskal-Wallis ANOVA results for multimodal scores by 5 redundancy levels

\begin{tabular}{|c|c|c|c|c|c|c|}
\hline$\overline{\text { Action }}$ & $\begin{array}{l}\text { Mean } \\
\text { Rank }\end{array}$ & Cases & $\begin{array}{l}\text { Overall } \\
\text { Redundancy } \\
\text { level }\end{array}$ & $\begin{array}{l}\text { Mean } \\
\text { Rank }\end{array}$ & Cases & $\begin{array}{l}\text { Detailed } \\
\text { Redundancy } \\
\text { level }\end{array}$ \\
\hline & 126.32 & 90 & $33 \%$ & 220.63 & 30 & $\bar{C} 33$ \\
\hline Cut, & & & & 95.07 & 30 & O33 \\
\hline Copy \& & & & & 63.25 & 30 & T33 \\
\hline \multirow[t]{4}{*}{ Paste } & 255.27 & 60 & $66 \%$ & 284.73 & 30 & $\mathrm{CO}$ \\
\hline & & & & 225.80 & 30 & CT \\
\hline & 171.08 & 90 & $25 \%$ & 201.92 & 30 & $\mathrm{C} 25$ \\
\hline & & & & 164.45 & 30 & $\mathrm{O} 25$ \\
\hline \multirow[t]{3}{*}{ Move } & & & & 146.87 & 30 & D25 \\
\hline & 147.59 & 60 & $75 \%$ & 163.12 & 30 & COT75 \\
\hline & & & & 132.07 & 30 & COD \\
\hline $\begin{array}{l}\text { Cut,Cop } \\
\text { yPaste }\end{array}$ & 234.05 & 60 & $100 \%$ & 281.92 & 30 & COT 100 \\
\hline \multirow[t]{3}{*}{ Move } & & & & 186.18 & 30 & COTD \\
\hline & $\begin{array}{l}\text { Chi- } \\
\text { square }\end{array}$ & D.F. & Sig. & $\begin{array}{l}\text { Chi- } \\
\text { square }\end{array}$ & D.F. & Sig. \\
\hline & 78.0482 & 4 & $\mathrm{p}<.0001$ & 139.6767 & 11 & $\mathrm{p}<.0001$ \\
\hline
\end{tabular}

Therefore, these preliminary results, while indicating that redundancy of information has a bearing on improving the input recognition task, also show that more complex requests (i.e. the move actions) appear to have presented particular recognition difficulties to the system. Further examination of the experimental data, to identify characteristics of the particular cases of interest, will form the next stage of analysis.

\subsection{Non-recognitions and Mis-recognitions}

Non-recognitions and mis-recognitions cases might be expected from the system. The integrator was programmed to make a best guess about the intended actions, based on the weightings given to the inputs by the speech and gesture systems. Due to the use of independent speech and gesture recognition systems, the rating mechanisms were different. Therefore, as well as being arbitrary within the individual systems, any comparison of the weights was also arbitrary. Hence it would be reasonable to expect this system to choose an option (based on the weights) that was incorrect, simply because the 'wrong' answer had the higher weighting.

Another factor in mis- and non-recognitions was the dependency of the complete multimodal combination on passing a total validity check. The validity check analysed the proposed multimodal combination in total, rather than considering possibilities slot by slot. Therefore, as the total multimodal combination had to be estimated to be correct, one or more slots that may have contained valid (and correct) values would have been discarded in the search for the best overall combination. Another possible validity check could have filled each slot with a possibility, checked that option for validity, and then attempted combinations of this slot with other slots, ending with a final validity check of the complete combination.

Further analysis of the data may reveal if these factors have adversely affected the data. Improvements to the integration algorithm may be possible through the modification of the validity check process, which in turn may improve upon some of the lower than expected multimodal recognition figures.

\section{Future Work}

A technique for the integration of speech and twodimensional gesture has been presented. Empirical data has shown that the redundant integration of these two modes provides multimodal recognition rates which improve upon either of the unimodal rates.

Further analysis has shown that particular levels of redundancy have an influence on multimodal recognition rates. Future work may indicate particular circumstances which have contributed to the effect. In addition, further work may identify specific factors which lead to the observed empirical gain in multimodal recognition rates.

Predictions of where and how these gains arise could produce guidelines and predictive models for the integration of multiple modes.

\section{References}

[1] Ando, H.; Kitahara, Y.; Hataoka, N.; Evaluation of Multimodal Interface using Spoken Language and Pointing Gesture on Interior Design System. International Conference on Spoken Language Processing, pp567-570, 1994.

[2] Billinghurst, M.; Savage, J.; Oppenheimer, P.; Edmond, C.; The Expert Surgical Assistant: An Intelligent Virtual Environment with Multimodal Input. Medicine meets Virtual Reality IV: Health Care in the Information Age, pp590-607, Amsterdam, IOS Press, 1996.

[3] Caloini; Tosolini, Paolo; Time and gesture based interfaces a test case: Shinjuku Guide. UK Toolbook User Conference, 1994.

[4] Cassell, J.; Steedman, M.; Badler, N.; Pelachaud, C.; Stone, M.; Douville, B.; Prevost, S.; Achorn, B.; Modeling the Interaction between Speech and Gesture. Proceedings of the 16th Annual Conference of the Cognitive Science Society, Georgia Institute of Technology, Atlanta, USA, 1994.

[5] Edwards, A.D.N.; Redundancy and Adaptability; Multimedia Interface Design in Education; Edwards, A.D.N. \& Holland, S. (eds.); ppl45-155, 1992.

[6] Hinde, C.J.; Bray, A.D.; Herbert, P.J.; Launders, V.A.; Round, D.; A Truth Maintenance Approach to Process Planning. Proceedings of the 4th International Conference 
on the Applications of Artificial Intelligence in Engineering, pp171-188, 1989.

[7] Koons, D.B.; Sparrell, C.J.; Thorisson, K.R.; Integrating Simultaneous Input from Speech, Gaze, and Hand Gestures. Intelligent Multimedia Interfaces, pp257-276, AAAI Press, 1993.

[8] Kramer, A.; Classifying Two Dimesional Gestures in Interactive Systems. Proceedings of the International Gesture Workshop, Bielefeld, Germany, pp37-48. Springer Lecture Notes in Artificial Intelligence, 1371,1997.

[9] McKenzie Mills, K.; Alty, J.L.; Investigating the Role of Redundancy. Proceedings of the International Gesture Workshop, Bielefeld, Germany, pp159-171. Springer Lecture Notes in Artificial Intelligence, 1371, 1997.

[10] Nakagawa, S.; Zhang, J.; Chengcharoen, W.; A multimodal Interface with Speech and Touch Screen. Symbiosis of Human and Artifact, pp213-218, Anzai; Ogawa; Mori, eds., 1995.

[!1] Oviatt, S.; DeAngeli, A.; Kuhn, K.; Integration and Synchronization of Input Modes during Multimodal HumanComputer Interaction.CHI 97, 22-27 March, pp.415-422, 1997.

[12] Oviatt, S.; Olsen, E.; Integration Themes in Multimodal Human-Computer Interaction. Proceedings of International Conference on Spoken Language Processing, Vol. 2, pp551$554,1994$.

[13] Schank, R.; Conceptual Dependency: A theory of natural language understanding. Cognitive Psychology 3, pp552-631, 1972.

[14] Vetere, F.; Redundancy in Mulimedia Systems. Proceedings of INTERACT '97, pp648-650, Chapman \& Hall, 1997.

[15] Vetere, F.; Howard, S.; Leung, Y.; A Multimedia Interaction Space. Proceedings of INTERACT '97, Chapman \& Hall, 1997.

[16] Vo, M.T.; Waibel, A.; A Multimodal HumanComputer Interface: Combination of Speech and Gesture Recognition. Adjunct Proceedings of InterCHI '93, 1993.

[17] DragonDictate Discrete Recognition Software, Dragon Systems,

1994. 
Appendix C 
